\title{
WestVirginiaUniversity
}

THE RESEARCH REPOSITORY @ WVU

Graduate Theses, Dissertations, and Problem Reports

2003

\section{Analysis of a curved buoyant jet in an enclosure using LES}

Allen E. Badeau Jr.

West Virginia University

Follow this and additional works at: https://researchrepository.wvu.edu/etd

\section{Recommended Citation}

Badeau, Allen E. Jr., "Analysis of a curved buoyant jet in an enclosure using LES" (2003). Graduate Theses, Dissertations, and Problem Reports. 2525.

https://researchrepository.wvu.edu/etd/2525

This Dissertation is protected by copyright and/or related rights. It has been brought to you by the The Research Repository @ WVU with permission from the rights-holder(s). You are free to use this Dissertation in any way that is permitted by the copyright and related rights legislation that applies to your use. For other uses you must obtain permission from the rights-holder(s) directly, unless additional rights are indicated by a Creative Commons license in the record and/ or on the work itself. This Dissertation has been accepted for inclusion in WVU Graduate Theses, Dissertations, and Problem Reports collection by an authorized administrator of The Research Repository @ WVU.

For more information, please contact researchrepository@mail.wvu.edu. 


\title{
ANALYSIS OF A CURVED BUOYANT JET IN AN ENCLOSURE USING LES
}

\author{
Allen E. Badeau Jr. \\ Dissertation submitted to the \\ College of Engineering and Mineral Resources \\ at West Virginia University \\ in partial fulfillment of the requirements \\ for the degree of \\ Doctor of Philosophy \\ in \\ Mechanical Engineering \\ Ismail B. Celik, Ph.D., Chair \\ John Kuhlman, Ph.D \\ Andrei Smirnov, Ph.D \\ David Lewellen, Ph.D \\ Ibrahim Yavuz, Ph.D \\ Peter Chang, Ph.D \\ Martin Ferer, Ph.D \\ Mechanical and Aerospace Engineering Department \\ Morgantown, West Virginia \\ 2003
}

Keywords: Large-eddy simulation, implicit turbulence modeling, curved buoyant jets Copyright 2003 


\section{ABSTRACT \\ ANALYSIS OF A CURVED BUOYANT JET IN AN ENCLOSURE USING LES}

Allen E. Badeau Jr.

The objective of this study is to investigate curved buoyant jets in an enclosure using Large Eddy Simulation (LES) methods with an Implicit Turbulent Model (ITM). To accomplish this goal, a numerical solver was written, named DREAM ${ }^{\circledR}$, which is capable of solving three dimensional, transient flows using an accurate monotonic and non-oscillatory upwinding scheme. The three-dimensional Navier-Stokes equations are solved in Cartesian coordinates, with the control volume approach being implemented on a staggered grid. The numerical scheme uses a fractional time step method, with the overall spatial and temporal accuracy being second order.

In ITM simulations, there is no explicit subgrid-scale model (SGS) used for the modeling of the small scale vortical structures. ITM simulations assume that through strict conservation of the fluxing quantities in and out of the cell, the grid resolution is fully capable of capturing the important scales of the flow. The volume averaging techniques used in the ITM methods acts as an implicit subgrid-scale model, and the resolvable scales of the flow are only dependent on the grid resolution within the domain. Comparison of the available experimental data, as well as simulations that used SGS models, to the ITM simulations from DREAM ${ }^{\circledR}$ compare favorably for most results.

For the simulations presented in this study, oil is injected at a specified flow rate into a water filled tank, initially taken to be stagnant. Results show that the density stratification tends to damp the amount of turbulence present within the jet near the interface, but overall increases turbulence because of the acceleration of the fuel. Analysis of the curved buoyant jet shows that at an appropriate downstream location, similarity is achieved, and the energy spectrum shows the appropriate inertial subrange characteristics. Impingement of the curved buoyant jet onto the upper wall increases the amount of turbulent present within the enclosure and comparison to vertical buoyant jet simulations with comparable dimensionless parameters shows wall effects may never be completely eliminated from the analysis. Comparison between the curved buoyant jet simulations to the available experimental data from experiments performed explicitly for this study shows good agreement for the buoyant path centerline locations based on the internal densimetric Froude number. The application of these methods to immiscible fluids shows a new dimension to ITM and allows for a high resolution of the resulting flow field without the need for an explicit SGS model. Simulations for the vertical and curved buoyant jet indicate the necessity for small timesteps and increased grid resolution. 


\section{ACKNOWLEGEMENTS}

This dissertation, as well as college, would never have been attempted without the help and constant support of my parents Allen and Karole Badeau. I will be forever indebted to them for all of their support. To my wonderful wife, Amy, who has put up with me for the last four years and helped me through all of the hard times. I only hope that my support of her is enough to compensate for the last few years.

I must also acknowledge my advisor, Dr. Ismail B. Celik, who gave me a chance when others were hesitant. His teaching and encouragement gave me the drive to succeed. His DREAM ${ }^{\circledR}$ is becoming a reality.

A great number of other people helped in the completion of this study. To Dave Frazer, who showed much patience while providing me time to complete my studies while working at NIOSH. To my undergraduate students, whom always were able to put things in perspective, and to the CFD lab, all of whom made my tenure at WVU fun and enjoyable.

It is with great pleasure that I also thank my committee members. I understand the commitment and greatly appreciate the time and input. Your help has shown me where I need to improve to become a better scientist. 


\section{TABLE OF CONTENTS}

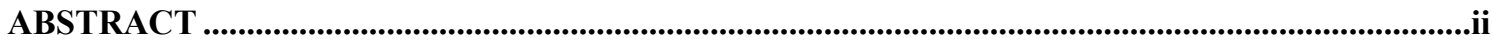

ACKNOWLEGEMENTS..........................................................................................................................ii

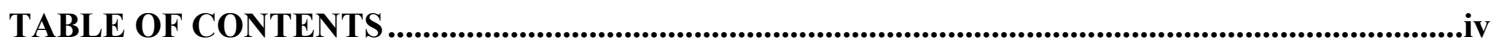

LIST OF TABLES................................................................................................................................

LIST OF FIGURES..............................................................................................................................................vii

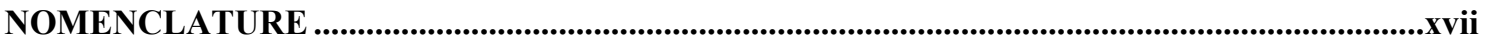

GREEK NOMENCLATURE ..........................................................................................................................

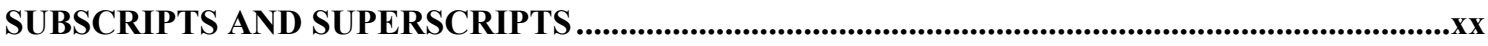

U.S. CUSTOMARY UNITS AND METRIC EQUIVALENTS...............................................................xxii

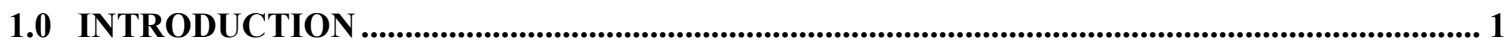

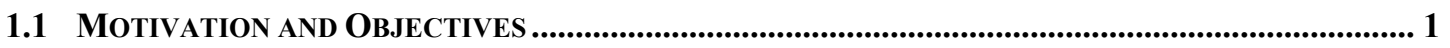

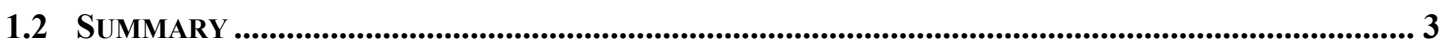

2.0 LITERATURE REVIEW ....................................................................................................... 5

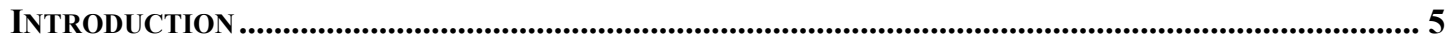

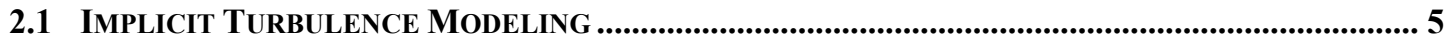

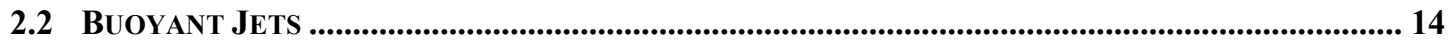

2.3 IMPINGEMENT OF BUOYANT JETS ON A NON-FREE SURFACE........................................................... 18

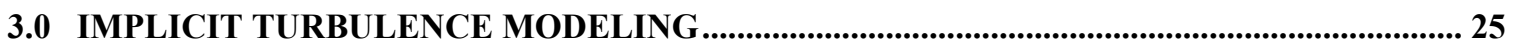

3.1 LES WITH IMPLICIT TURBULENCE MODELING TECHNIQUES ........................................................ 25

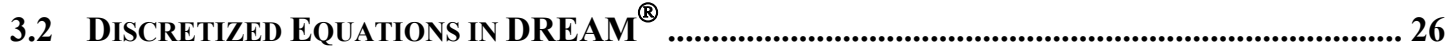

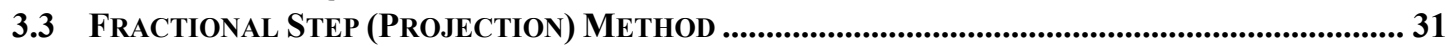

3.4 SOLUTION OF SYSTEM OF ALGEBRAIC EQUATIONS............................................................................ 33

3.5 BOUNDARY CONDITIONS ............................................................................................................................... 34

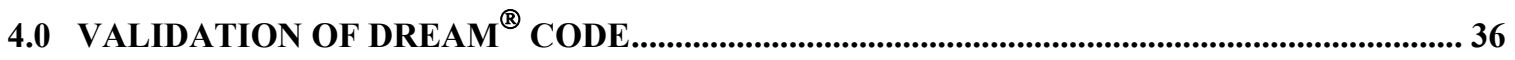

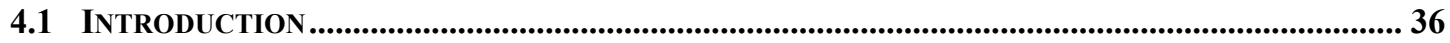

4.2 LES OF FLOW PAST A SQUARE CYLINDER ................................................................................ 36

4.3 LES OF PLANE MIXING LAYER ................................................................................................. 43

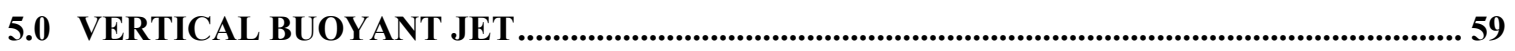

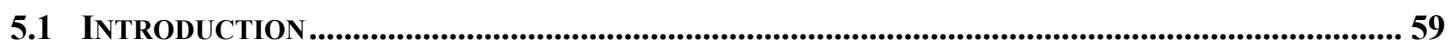

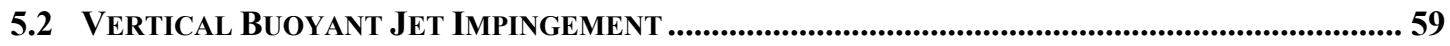

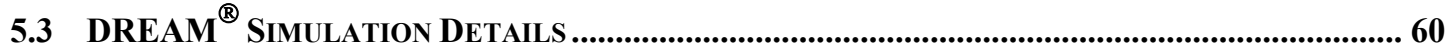

5.4 RESULTS AND DISCUSSION ....................................................................................................................... 62

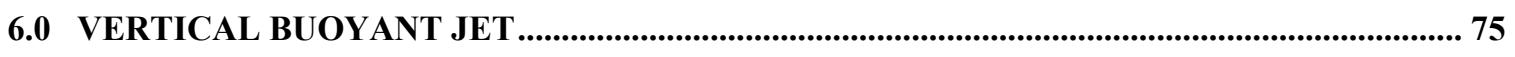

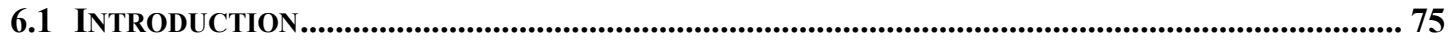

6.2 DESCRIPTION OF EXPERIMENTS ..................................................................................................... 75 
6.2.1 Vertical Buoyant Jet ....................................................................................................................... 75

6.2.2 Vertical Buoyant Jet with Axisymmetric Forcing .............................................................. 77

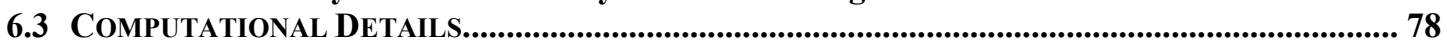

6.4 RESULTS AND CONCLUSIONS .............................................................................................................. 79

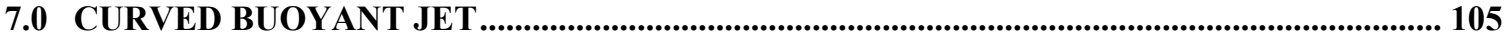

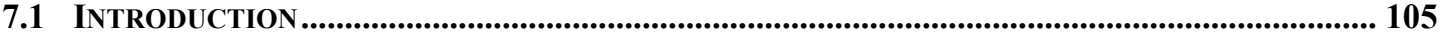

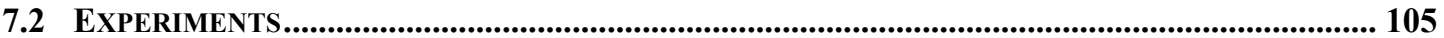

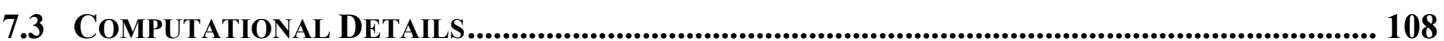

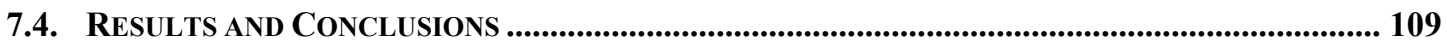

7.4.1 Curved Buoyant Jet Properties ...................................................................................................... 110

7.4.2 Global Effects of the Buoyant Jet................................................................................. 115

8.0 CONCLUSIONS AND FUTURE RECOMMENDATIONS ................................................... 147

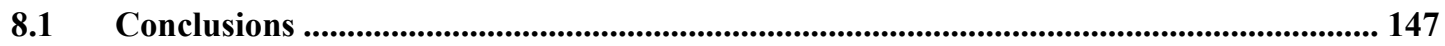

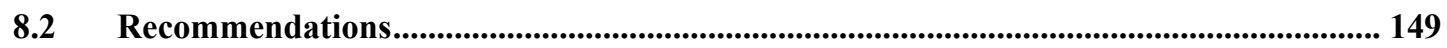

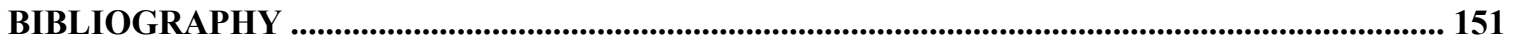

APPENDIX A - VERIFICATION OF SCALAR SOLVER.................................................................... 155

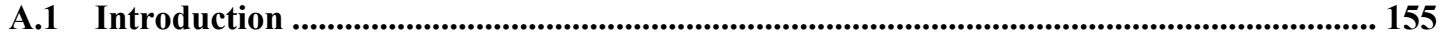

A.2 Literature Review of QSOU .................................................................................................. 155

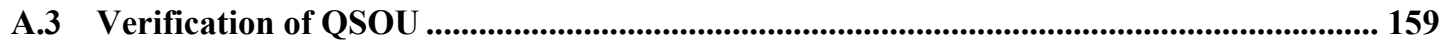

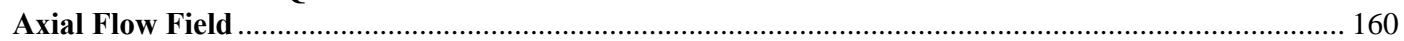

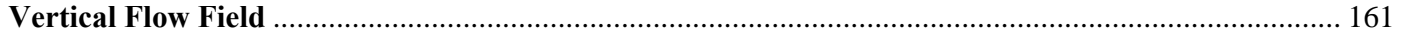

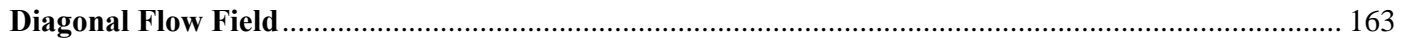

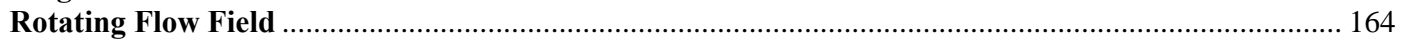

A.4 Verification of Variable Density Scheme (Conservation of Mass)........................................ 167

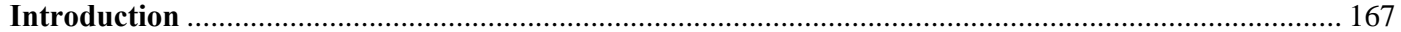

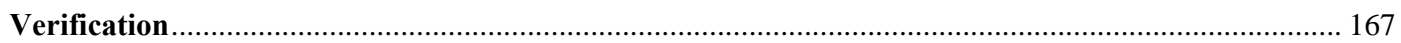

APPENDIX B - INTRODUCTION TO DREAM ${ }^{\circledR}$ CODE …......................................................... 173

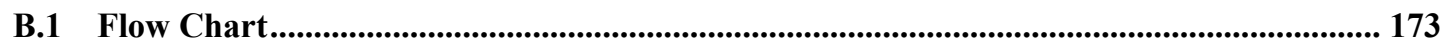

B.2 Inclusion of wall and baffles within domain ................................................................... 174

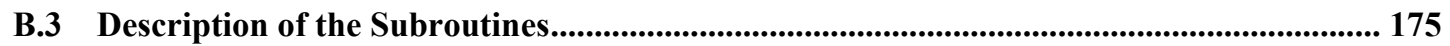

INDEX ..................................................................................................................................................... 177 


\section{LIST OF TABLES}

Table 5.3.1 - Simulation parameters from buoyant jet experiments ................59

Table 7.2.1 - Experimental parameters for buoyant jet experiments ............... 98

Table 7.3.1 - Experimental parameters for buoyant jet experiments ............... 99 


\section{LIST OF FIGURES}

Figure 3.2.1 - Grid used for integration of transport equations .................................. 28

Figure 3.3.1 - Staggered grid used in the DREAM ${ }^{\circledR}$ code ...................................... 32

Figure 4.2.1 - The geometry of the flow past a square cylinder ................................. 37

Figure 4.2.2 - Mean streamwise velocity distribution on the center plane of the cylinder. The experiments are by Lyn et al. (1994) and the data starts behind the cylinder. The cylinder is located between $-1<\mathrm{x} / \mathrm{D}<1$. (a) DREAM ${ }^{\circledR}$ simulations (b) The corresponding simulations are from Shi (2001) using the QUICK scheme with the Smagorinsky SGS model. 39

Figure 4.2.3 - Streamwise velocity component of the turbulence intensity of the flow past a square cylinder. (a) The corresponding simulations are from Shi (2001) using the QUICK scheme with Smagorinsky SGS model. (b) DREAM ${ }^{\circledR}$ simulations

Figure 4.2.4 - Vertical velocity component of the turbulence intensity of the flow past a square cylinder. (a) The corresponding simulations are from Shi (2001) using the QUICK scheme with Smagorinsky SGS model. (b) DREAM ${ }^{\circledR}$ simulations 41

Figure 4.2.5 - $\mathrm{z}$ component vorticity of the flow past a square cylinder for the DREAM ${ }^{\circledR}$ simulations. 42

Figure 4.2.6 - Center plane streamlines for the flow past a square cylinder using DREAM $^{\circledR}$ 42

Figure 4.3.1 - Schematic of plane mixing layer domain (Shi (2001)) 43

Figure 4.3.2 - Velocity vectors at $x / \lambda=1.25$ (a) Measurements by Rightley (1995); (b) Simulations by Shi (2001); (c) Present DREAM ${ }^{\circledR}$ simulations (300 x 120 x 60).. 48

Figure 4.3.3 - Velocity vectors at $x / \lambda=1.88$ (a) Measurements by Rightley (1995); (b) Simulations by Shi (2001); (c) Present DREAM ${ }^{\circledR}$ simulations (300 x 120 x 60).. 49

Figure 4.3.4 - Velocity vectors at $x / \lambda=2.5$ (a) Measurements by Rightley (1995); (b) Simulations by Shi (2001); (c) Present DREAM ${ }^{\circledR}$ simulations (300 x 120 x 60).. 50

Figure 4.3.5 - Vertical velocity contours from Shi (2001)........................................ 51

Figure 4.3.6 - Vertical velocity contours from DREAM ${ }^{\circledR}(300 \times 120 \times 60) \ldots . . . . . . . . . .51$ 
Figure 4.3.7 - (a) Mean and instantaneous velocities at $\mathrm{x} / \lambda=1.25$ from DREAM ${ }^{\circledR}$ simulations within the mixing region. Dashed lines are mean velocities at $y=0.185$ : -----; and $y=0.210$; Solid lines are at the same locations, and represent the instantaneous velocities; (b) Zoomed in view of the $y=0.210$ profile showing turbulence fluctuations in velocity; (c) Zoomed in view of the $y=0.185$ profile...... 52

Figure 4.3.8 - Mean and $r m s$ streamwise velocity at $x / \lambda=1.25$. Symbols are from Rightley (1995): $\diamond$ : mean streamwise velocity; 0 : $\mathrm{rms}$ of streamwise velocity fluctuations. Lines from Shi (2001): ___ _ : mean streamwise velocity; -----: rms of streamwise velocity fluctuations.

Figure 4.3.9 - Mean and $r m s$ streamwise velocity at $x / \lambda=1.25$. Symbols are from Rightley (1995): $\diamond$ : mean streamwise velocity; 0 : $r m s$ of streamwise velocity fluctuations. Lines : : mean streamwise velocity from DREAM ${ }^{\circledR}$ simulations (200x80x40); -----: rms of streamwise velocity fluctuations from DREAM ${ }^{\circledR}$ simulations $(200 \times 80 \times 40)$. 53

Figure 4.3.10 - Mean and $r m s$ streamwise velocity at $x / \lambda=1.25$. Symbols are from Rightley (1995): $\diamond$ : mean streamwise velocity; o: rms of streamwise velocity fluctuations. Lines : : mean streamwise velocity from DREAM ${ }^{\circledR}$ simulations (300x120x60); -----: rms of streamwise velocity fluctuations from DREAM ${ }^{\circledR}$ simulations $(300 \times 120 \times 60)$.

Figure 4.3.11 - Mean and $r m s$ vertical velocity at $x / \lambda=1.25$. Symbols are from Rightley (1995): $\diamond$ : mean vertical velocity; $0:$ rms of vertical velocity fluctuations. Lines from Shi (2001): : mean vertical velocity; -----: rms of vertical velocity

fluctuations. 54

Figure 4.3.12 - Mean and $r m s$ vertical velocity at $x / \lambda=1.25$. Symbols are from Rightley (1995): $\diamond$ : mean vertical velocity; 0 : $\mathbf{r m s}$ of vertical velocity fluctuations. Lines from DREAM ${ }^{\circledR}$ Simulations: : mean vertical velocity $(200 \times 80 \times 40)$; ----: $r m s$ of vertical velocity fluctuations $(200 \times 80 \times 40)$. 55

Figure 4.3.13 - Mean and rms vertical velocity at $x / \lambda=1.25$. Symbols are from Rightley (1995): $\diamond$ : mean vertical velocity; 0 : $\mathbf{r m s}$ of vertical velocity fluctuations. Lines from DREAM ${ }^{\circledR}$ Simulations: ___ _ _ mean vertical velocity (300x120x40); ----: rms of vertical velocity fluctuations $(300 \times 120 \times 60)$. 55

Figure 4.3.14 - Turbulent kinetic energy comparison at $x / \lambda=1.25$. Symbols represent approximation from Rightley (1995) $\mathbf{~ r m s ~ d a t a : ~} \square$; --- Dashed lines represent resolved portion of turbulent kinetic energy from DREAM simulations for 200x80x40 grid; Solid lines representing only the resolved portion of the turbulent kinetic energy from the DREAM ${ }^{\circledR}$ simulations for 300x120x60 grid size. Resolved portion is approximately $85 \%$ of experimental data, which is good for LES simulations. 56 
Figure 4.3.15 - Energy spectra for the a) streamwise and b) vertical velocity components at $x / \lambda=1.25$ (Rightley, 1995)

Figure 4.3.16 - Averaged energy spectra for the a) streamwise and b) vertical

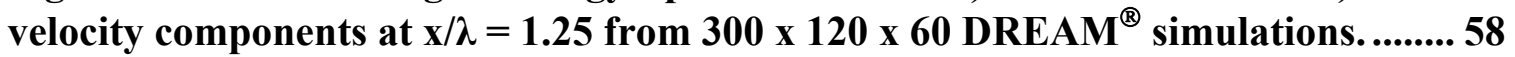

Figure 5.1.a - Experimental fuel impingement test facility used at The Johns Hopkins University by Friedman and Katz (1999).............................................. 66

Figure 5.1.b - Center plane view of the geometry used in the DREAM ${ }^{\circledR}$ simulations 66

Figure 5.2 - Experimental impinging jet instantaneous picture for Regime 3 showing initial water jet impingement onto upper fuel layer for $R i=0.08$; cavity is symmetric.

Figure 5.3 - Density contour from DREAM ${ }^{\circledR}$ simulations for $\mathrm{Ri}=\mathbf{0 . 0 8}$ using $50 \times 157 \times 157$ grid size with $d t=0.001$ seconds; $t=0.4$ seconds after impact

Figure 5.4 - Density contour from DREAM ${ }^{\circledR}$ simulations for $\mathrm{Ri}=\mathbf{0 . 0 8}$ using $100 \times 213 \times 213$ grid size with $d t=0.001$ seconds; $t=0.4$ seconds after impact.

Figure 5.5 - Comparison of DREAM ${ }^{\circledR}$ simulations using 50x157x157 and $100 \times 213 \times 213$ grid sizes to experimental maximum penetration depth $(A R)$ data; $d t=$ 0.001 seconds. 68

Figure 5.6 - (a) Experimental impinging jet experiment for Regime 3 showing onset of water jet instability for $R i=0.08, t=1.2$ seconds after initial jet impingement. DREAM $^{\circledR}$ simulations for $\mathrm{Ri}=\mathbf{0 . 0 8}$ using (b) $50 \times 157 \times 157, t=1.22$ seconds after initial jet impingement, and (c) $100 \times 213 \times 213$ grid size showing onset of water jet instability, $t=1.205$ seconds after initial jet impingement. 69

Figure 5.7 - Instantaneous normalized $z$ vorticity contours, with vectors, for $\mathbf{R} \mathbf{i}=$ 0.08 using 50x157x157 grid size; $d t=0.001 ; t=1.2$ seconds after initial impact...... 70

Figure 5.8 - Instantaneous normalized $z$ vorticity contours, with vectors, for $\mathbf{R i}=$ 0.08 using $100 \times 207 \times 207$ grid size; $d t=0.001 ; t=1.2$ seconds after initial impact... 70

Figure 5.9 - Resolved RMS for $\mathrm{Ri}=0.08$ using $100 \times 213 \times 213$ grid size at $\mathrm{y}=0.5$; $\mathrm{dt}=$ 0.001 seconds.

Figure 5.10 - Resolved RMS for $\mathbf{R i}=\mathbf{0 . 0 8}$ using $100 \times 213 \times 213$ grid size at $\mathbf{y}=\mathbf{0 . 5 2 5}$; dt $=0.001$ seconds. 
Figure 5.11 - Normalized resolved turbulent kinetic energy for $\mathbf{R i}=\mathbf{0 . 0 8}$ using $100 \times 213 \times 213$ grid size at $\mathrm{y}=0.5$ and $\mathrm{y}=0.525 ; \mathrm{dt}=0.001$ seconds. Jet inlet is located at $x / D_{\text {in }}=5$. 72

Figure 5.12 - Energy spectrum for $\mathrm{Ri}=0.08$ using $100 \times 213 \times 213$ grid size at $\mathrm{y}=\mathbf{0 . 5}$; dt $=0.001$ seconds 73

Figure 5.13 - Energy spectrum for $\mathrm{Ri}=0.08$ using $100 \times 213 \times 213$ grid size at $\mathrm{y}=$ $0.525 ; \mathrm{dt}=0.001$ seconds. 73

Figure $5.14-$ Normal stresses for $R i=0.08$ using $100 \times 213 \times 213$ grid size at $x=0.175$ across the jet; $\mathrm{dt}=\mathbf{0 . 0 0 1}$ seconds. Averaging time $=18.0$ seconds.

Figure 5.15 - Normalized resolved turbulent kinetic energy for $\mathrm{Ri}=\mathbf{0 . 0 8}$ using $100 \times 213 \times 213$ grid size at $x=0.175$ across the jet; $d t=0.001$ seconds. Total averaging time $=18.0$ seconds. 74

Figure 6.1 - Geometry used in Anwar (1968) experiments....................................... 83

Figure 6.2 - Geometry used in the George and Tamanini (1977) experiments......... 83

Figure 6.3 - Coarse grid used in the DREAM ${ }^{\circledR}$ simulations for Anwar (1964) experiments

Figure 6.4 - Fine grid used in the DREAM ${ }^{\circledR}$ simulations for comparison to Anwar (1964) experiments

Figure 6.5 - Velocity magnitude contours at $\mathrm{z}=0$ for $75 \times 57 \times 57$ grid resolution after four flow through times; $\mathrm{dt}=0.00025 ; \mathrm{Re}=1892 ; \mathrm{Fr}=2.8$ 85

Figure 6.6 - Velocity magnitude contours at $z=0$ for $150 \times 107 \times 107$ grid resolution after four flow through times; $\mathrm{dt}=0.00025 ; \mathrm{Re}=1892 ; \mathrm{Fr}=2.8$ 85

Figure 6.7 - Instantaneous velocity vectors at $\mathrm{z}=0$ for $75 \times 57 \times 57$ grid resolution after four flow through times; $\mathrm{dt}=0.00025 ; \mathrm{Re}=1892 ; \mathrm{Fr}=2.8$ 86

Figure 6.8 - Instantaneous velocity vectors at $\mathrm{z}=0$ for $150 \times 107 \times 107$ grid resolution after four flow through times; $\mathrm{dt}=0.00025 ; \mathrm{Re}=1892 ; \mathrm{Fr}=2.8$ 86

Figure 6.9 - Instantaneous point velocity component versus time for $75 \times 57 \times 57$ grid resolution; $\mathrm{dt}=0.00025 ; ; \operatorname{Re}=1892 ; \mathrm{Fr}=2.8$.

Figure 6.10 - Instantaneous point velocity component versus time for $150 \times 107 \times 107$ grid resolution; $\mathrm{dt}=\mathbf{0 . 0 0 0 2 5} ; \mathrm{Re}=1892 ; \mathrm{Fr}=2.8$ 
Figure 6.11 - $Z$ component vorticity contours at $z=0$ for $75 \times 57 \times 57$ grid resolution after four flow through times; $\mathrm{dt}=0.00025 ; \mathrm{Re}=1892 ; \mathrm{Fr}=2.8$ 88

Figure 6.12 - $Z$ component vorticity contours at $z=0$ for $150 \times 107 \times 107$ grid resolution after four flow through times; $\mathrm{dt}=\mathbf{0 . 0 0 0 2 5} ; \operatorname{Re}=1892 ; \mathrm{Fr}=2.8$ 88

Figure 6.13 - Vorticity magnitude contours at $\mathrm{z}=0$ for $75 \times 57 \times 57$ grid resolution after four flow through times; $\mathrm{dt}=0.00025 ; \mathrm{Re}=1892 ; \mathrm{Fr}=2.8$ 89

Figure 6.14 - Vorticity magnitude contours at $\mathrm{z}=0$ for $150 \times 107 \times 107$ grid resolution after four flow through times; $\mathrm{dt}=0.00025 ; \mathrm{Re}=1892 ; \mathrm{Fr}=2.8$ 89

Figure 6.15 - Density contours at $\mathrm{z}=0$ for $75 \times 57 \times 57$ grid resolution after four flow through times; $\mathrm{dt}=0.00025 ; \mathrm{Re}=1892 ; \mathrm{Fr}=2.8$ 90

Figure 6.16 - Density contours at $\mathrm{z}=0$ for $150 \times 107 \times 107$ grid resolution after four flow through times; $\mathrm{dt}=0.00025 ; \mathrm{Re}=1892 ; \mathrm{Fr}=2.8$... 90

Figure 6.17 - Normalized axial velocity at $x / D_{\text {in }}=15$ and $x / D_{\text {in }}=20$ from DREAM ${ }^{\circledR}$ simulations to experiments; $\mathrm{dt}=0.00025 ; \mathrm{Re}=1892 ; \mathrm{Fr}=2.8$ 91

Figure 6.18 - Comparison of $U_{\text {rms }}$ from DREAM ${ }^{\circledR}$ simulations to experiments at $x / D_{\text {in }}=15$ and $x / D_{\text {in }}=20 ; \mathrm{dt}=0.00025 ; \operatorname{Re}=1892 ; \mathrm{Fr}=2.8$.

Figure 6.19 - Comparison of $V_{\text {rms }}$ from DREAM ${ }^{\circledR}$ simulations to experiments at $x / D_{\text {in }}=15$ and $x / D_{\text {in }}=20 ; d t=0.00025 ; \operatorname{Re}=1892 ; \mathrm{Fr}=2.8$

Figure 6.20 - Comparison of $W_{\text {rms }}$ from DREAM ${ }^{\circledR}$ simulations to experiments at $x / D_{\text {in }}=15$ and $x / D_{\text {in }}=20 ; \mathrm{dt}=0.00025 ; \operatorname{Re}=1892 ; \mathrm{Fr}=2.8$. 92

Figure 6.21 - Comparison of total energy spectrum at $\mathrm{x} / \mathrm{Din}=15$ from DREAM ${ }^{\circledR}$ simulations using coarse and fine grid simulations 93

Figure 6.22 - Comparison of total energy spectrum at $\mathrm{x} / \mathrm{Din}=20$ from DREAM ${ }^{\circledR}$ simulations using coarse and fine grid simulations

Figure 6.23 - Coarse grid used in DREAM ${ }^{\circledR}$ simulations for George and Tamanini (1977) experiments 95

Figure 6.24 - Coarse grid used in DREAM ${ }^{\circledR}$ simulations for George and Tamanini (1977) experiments 95

Figure 6.25 - Velocity magnitude contours at $\mathrm{z}=0$ for $75 \times 57 \times 57$ grid resolution after four flow through times; $d t=0.00025 ; \operatorname{Re}=1273 ; \mathrm{Fr}=1.93$. 96 
Figure 6.26 - Velocity magnitude contours at $z=0$ for $150 \times 107 \times 107$ grid resolution after four flow through times; $d t=0.00025 ; \operatorname{Re}=1273 ; \mathrm{Fr}=1.93$ 96

Figure 6.27 - Instantaneous velocity vectors at $\mathrm{z}=0$ for $75 \times 57 \times 57$ grid resolution after four flow through times; $\mathrm{dt}=0.00025 ; \mathrm{Re}=1273 ; \mathrm{Fr}=1.93$

Figure 6.28 - Instantaneous velocity vectors at $z=0$ for $150 \times 107 \times 107$ grid resolution after four flow through times; $\mathrm{dt}=\mathbf{0 . 0 0 0 2 5} ; \mathrm{Re}=1273 ; \mathrm{Fr}=1.93$ 97

Figure $6.29-Z$ component vorticity contours at $z=0$ for $75 \times 57 \times 57$ grid resolution after four flow through times; $\mathrm{dt}=0.00025 ; \operatorname{Re}=1273 ; \mathrm{Fr}=1.93$ 98

Figure 6.30 - $Z$ component vorticity contours at $z=0$ for $150 \times 107 \times 107$ grid resolution after four flow through times; $\mathrm{dt}=0.00025 ; \operatorname{Re}=1273 ; \mathrm{Fr}=1.93$ 98

Figure 6.31 - Vorticity magnitude contours at $z=0$ for $75 \times 57 \times 57$ grid resolution after four flow through times; $\mathrm{dt}=0.00025 ; \mathrm{Re}=1273$; $\mathrm{Fr}=1.93$ 99

Figure 6.32 - Vorticity magnitude contours at $\mathrm{z}=0$ for $150 \times 107 \times 107$ grid resolution after four flow through times; $\mathrm{dt}=0.00025 ; \operatorname{Re}=1273 ; \mathrm{Fr}=1.93$ 99

Figure 6.33 - Density contours at $\mathrm{z}=0$ for $75 \times 57 \times 57$ grid resolution after four flow through times; $\mathrm{dt}=0.00025 ; \operatorname{Re}=1273 ; \mathrm{Fr}=1.93$ 100

Figure 6.34 - Density contours at $z=0$ for $150 \times 107 \times 107$ grid resolution after four flow through times; $d t=0.00025 ; \operatorname{Re}=1273 ; \mathrm{Fr}=1.93$ 100

Figure 6.35 - Normalized axial velocity across the jet at $x / D_{\text {in }}=8$ and $x / D_{\text {in }}=14$ from DREAM ${ }^{\circledR}$ simulations as compared to experiments; $\mathrm{dt}=0.00025 ; \mathrm{Re}=1273$; Fr $=1.93$ 101

Figure 6.36 - Comparison of $U_{\text {rms }}$ from DREAM ${ }^{\circledR}$ simulations to experiments at $x / D_{\text {in }}=8$ and $x / D_{\text {in }}=14 ; \mathrm{dt}=0.00025 ; \operatorname{Re}=1273 ; \mathrm{Fr}=1.93$.

Figure 6.37 - Comparison of $\mathrm{V}_{\text {rms }}$ from DREAM ${ }^{\circledR}$ simulations to experiments at $x / D_{\text {in }}=8$ and $x / D_{\text {in }}=14 ; d t=0.00025 ; \operatorname{Re}=1273 ; F r=1.93$

Figure 6.38 - Comparison of $W_{\text {rms }}$ from DREAM ${ }^{\circledR}$ simulations to experiments at $x / D_{\text {in }}=8$ and $x / D_{\text {in }}=14 ; \mathrm{dt}=0.00025 ; \operatorname{Re}=1273 ; \mathrm{Fr}=1.93$.

Figure 6.39 - Comparison of resolved density fluctuations from DREAM ${ }^{\circledR}$ simula tions between to experimental data at $x / D_{\text {in }}=8$ and $x / D_{\text {in }}=14 ; \operatorname{Re}=1273 ; \mathrm{Fr}=$ 1.93 . 
Figure 6.40 - Comparison of resolved turbulent kinetic energy from DREAM ${ }^{\circledR}$ simulations between to experimental data at $x / D_{\text {in }}=8$ and $x / D_{\text {in }}=14 ; \operatorname{Re}=1273 ; \mathrm{Fr}$ $=1.93$ 103

Figure 6.41 - Comparison of total energy spectrum at $\mathrm{x} / \mathrm{D}_{\mathrm{in}}=14$ from DREAM ${ }^{\circledR}$ simulations using coarse and fine grid simulations; $\operatorname{Re}=1273 ; \mathrm{Fr}=1.93$

Figure 7.1 - Experimental setup used in the validation of DREAM ${ }^{\circledR}$ code 120

Figure 7.2 - Locations where jet data was obtained for similarity analysis 121

Figure 7.3 - Mean velocity profile within jet region at $y / D_{\text {in }}=15.0$ and 20.0 within the similarity regions of jet compared to experimental data of Hussein et. al. (1994) (from Pope, 2000). $U_{0}$ is the inlet velocity.

Figure 7.4 - Turbulence intensity squared from DREAM ${ }^{\circledR}$ simulations within jet region at $\mathrm{x} / \mathrm{Din}=20$ for $240 \times 160 \times 80$ grid compared to experimental data from Hussein et. al. (1994). 122

Figure 7.5 - Normalized resolved turbulent kinetic energy in half jet within the selfsimilar region of $y / D_{i n}=20$ for $240 \times 160 \times 80$ grid; $d t=0.001$ compared to experimental data from Hussein et. al. (1994). 122

Figure 7.6 - Energy spectra for (a) the streamwise component and (b) density fluctuations in half jet within the similitude region of $y / D_{i n}=20$ for $240 \times 160 \times 80$ grid; $\mathbf{d t}=\mathbf{0 . 0 0 1}$ sec.

Figure 7.7 - (a) Experimental picture of inverted curved buoyant jet for $U_{\text {inlet }}=0.25$ $\mathrm{m} / \mathrm{sec} ; \mathrm{t}=18.0$ seconds, (b) DREAM ${ }^{\circledR}$ density contour for $240 \times 160 \times 80$ grid for $U_{\text {inlet }}$ $=0.25 \mathrm{~m} / \mathrm{sec}$; $\mathrm{dt}=0.001 ; \mathrm{t}=18.0$ seconds; $\mathrm{Fr}=1.91$. 124

Figure 7.8 - a) Experimental picture of curved buoyant jet for $U_{\text {inlet }}=1.0 \mathrm{~m} / \mathrm{sec}$ (inverted for comparative purposes). b) DREAM ${ }^{\circledR}$ density centerline contour for $240 \times 160 \times 80$ grid for $U_{\text {inlet }}=1.0 \mathrm{~m} / \mathrm{sec}$; $\mathrm{dt}=0.001 ; \mathrm{t}=5.0$ seconds; $\mathrm{Fr}=7.97$. 125

Figure 7.9 - Comparison of densimetric Froude number dependence for curved buoyant jet centerline path between experiments and DREAM ${ }^{\circledR}$ simulations for 240x160x80 grids; $d t=0.001$ sec. Error bars represent maximum variations between the sixteen different experiments. 126

Figure 7.10 - Typical instantaneous velocity magnitude contours for $240 \times 160 \times 80$ grid; $\mathrm{dt}=0.001 ; \mathrm{U}_{\text {inlet }}=0.25 \mathrm{~m} / \mathrm{sec} ; \mathrm{t}^{*}=0.1875 ; \mathrm{Fr}=1.91$

Figure 7.11 - Typical instantaneous axial velocity contours for $240 \times 160 \times 80$ grid; $\mathrm{dt}=$ $0.001 ; U_{\text {inlet }}=0.5 \mathrm{~m} / \mathrm{sec} ; \mathrm{t}^{*}=0.25 ; \mathrm{Fr}=3.83$. 
Figure 7.12 - Typical instantaneous velocity magnitude contours for $240 \times 160 \times 80$ grid; $d t=0.001 ; U_{\text {inlet }}=1.0 \mathrm{~m} / \mathrm{sec} ; \mathrm{t}^{*}=0.15 ; \mathrm{Fr}=7.65$

Figure 7.13 - Typical instantaneous vorticity magnitude contours for $240 \times 160 \times 80$ grid; $\mathrm{dt}=0.001 ; \mathrm{U}_{\text {inlet }}=0.25 \mathrm{~m} / \mathrm{sec} ; \mathrm{t}^{*}=0.1875 ; \mathrm{Fr}=1.91$

Figure 7.14 - Typical instantaneous vorticity magnitude contours for $240 \times 160 \times 80$ grid; $\mathrm{dt}=0.001 ; \mathrm{U}_{\text {inlet }}=0.5 \mathrm{~m} / \mathrm{sec} ; \mathrm{t}^{*}=0.25 ; \mathrm{Fr}=3.83$

Figure 7.15 - Typical instantaneous vorticity magnitude contours for $240 \times 160 \times 80$ grid; $\mathrm{dt}=0.001 ; \mathrm{U}_{\text {inlet }}=1.0 \mathrm{~m} / \mathrm{sec} ; \mathrm{t}^{*}=0.15 ; \mathrm{Fr}=7.65$

Figure 7.16 - Typical instantaneous velocity vectors (colored by span wise velocity component) for $240 \times 160 \times 80$ grid; $d t=0.001 ; U_{\text {inlet }}=0.25 \mathrm{~m} / \mathrm{sec} ; \mathrm{t}^{*}=0.2975 ; \mathrm{Fr}=$ 1.91 130

Figure 7.17 - Typical instantaneous velocity vectors for $240 \times 160 \times 80$ grid; $\mathrm{dt}=0.001$; $U_{\text {inlet }}=0.25 \mathrm{~m} / \mathrm{sec} ; t^{*}=0.0982 ; \mathrm{Fr}=1.91$ 130

Figure 7.18 - Typical instantaneous velocity vectors (colored by span wise velocity component) for $240 \times 160 \times 80$ grid; $d t=0.001 ; U_{\text {inlet }}=0.5 \mathrm{~m} / \mathrm{sec} ; \mathrm{t}^{*}=0.3875 ; \mathrm{Fr}=3.83$

Figure 7.19 - Typical instantaneous velocity vectors for $240 \times 160 \times 80$ grid; $\mathrm{dt}=0.001$; $U_{\text {inlet }}=0.5 \mathrm{~m} / \mathrm{sec} ; \mathrm{t}^{*}=0.1075 ; \mathrm{Fr}=3.83$.

Figure 7.20 - Typical instantaneous velocity vectors (colored by span wise velocity component) for $240 \times 160 \times 80$ grid; $d t=0.001 ; U_{\text {inlet }}=1.0 \mathrm{~m} / \mathrm{sec} ; t^{*}=0.15 ; \mathrm{Fr}=7.65$

Figure 7.21 - Typical instantaneous velocity vectors for $240 \times 160 \times 80$ grid; $\mathrm{dt}=0.001$; $U_{\text {inlet }}=1.0 \mathrm{~m} / \mathrm{sec} ; \mathrm{t}^{*}=0.085 ; \mathrm{Fr}=7.65$

Figure 7.22 - Typical instantaneous density contours for $240 \times 160 \times 80$ grid; $\mathrm{dt}=$ $0.001 ; U_{\text {inlet }}=0.25 \mathrm{~m} / \mathrm{sec} ; \mathrm{t}^{*}=\mathbf{0 . 1 0 8 5} ; \mathrm{Fr}=1.91$

Figure 7.23 - Typical instantaneous density contours for 240x160x80 grid; dt = $0.001 ; U_{\text {inlet }}=0.5 \mathrm{~m} / \mathrm{sec} ; \mathrm{t}^{*}=0.275 ; \mathrm{Fr}=3.63$

Figure 7.24 - Typical instantaneous density contours for $240 \times 160 \times 80$ grid; $\mathrm{dt}=$ $0.001 ; U_{\text {inlet }}=1.0 \mathrm{~m} / \mathrm{sec} ; t^{*}=0.263$ seconds; $F r=7.65$. 134

Figure 7.25 - Average velocity profiles from DREAM ${ }^{\circledR}$ simulations at $x=0.15 \mathrm{~m}$ and $0.30 \mathrm{~m}$ using $180 \times 120 \times 60$ and $240 \times 160 \times 80$ for a) axial velocity component, b) vertical velocity component, and c) spanwise velocity component; $d t=0.001 ; U_{\text {inlet }}=1.0$ $\mathrm{m} / \mathrm{sec} ; \mathrm{Fr}=7.65$ 135 
Figure 7.26 - Richardson extrapolation for average axial velocity profiles at $x=0.30$ $m$ for $120 \times 80 \times 40,180 \times 120 \times 60$ and $240 \times 160 \times 80$ grids; $d t=0.001 ; U_{\text {inlet }}=1.0 \mathrm{~m} / \mathrm{sec}$; $\mathrm{Fr}=7.65$

Figure 7.27 - Average velocity profiles from DREAM ${ }^{\circledR}$ simulations at $\mathbf{y}=\mathbf{0 . 2} \mathrm{m}$ and $0.4 \mathrm{~m}$ using $180 \times 120 \times 60$ and $240 \times 160 \times 80$ for a) axial velocity component, b) vertical velocity component, and c) spanwise velocity component; $\mathrm{dt}=0.001 ; U_{\text {inlet }}=1.0$ $\mathrm{m} / \mathrm{sec} ; \mathrm{Fr}=7.65$

Figure 7.28 - Mean RMS velocity profiles from DREAM ${ }^{\circledR}$ simulations at $\mathbf{x}=0.15 \mathrm{~m}$ and $0.30 \mathrm{~m}$ using $180 \times 120 \times 60$ and $240 \times 160 \times 80$ for a) axial velocity component, b) vertical velocity component, and c) spanwise velocity component; $d t=0.001 ; U_{\text {inlet }}=$ $1.0 \mathrm{~m} / \mathrm{sec} ; \mathrm{Fr}=7.65$ 138

Figure 7.29 - Mean RMS velocity profiles from DREAM ${ }^{\circledR}$ simulations at $\mathbf{y}=0.2 \mathrm{~m}$ and $0.40 \mathrm{~m}$ using $180 \times 120 \times 60$ and $240 \times 160 \times 80$ for a) axial velocity component, b) vertical velocity component, and c) spanwise velocity component; $d t=0.001 ; U_{\text {inlet }}=$ $1.0 \mathrm{~m} / \mathrm{sec} ; \mathrm{Fr}=7.65$ 139

Figure 7.30 - Square of the turbulence intensity from DREAM ${ }^{\circledR}$ simulations at $x=$ $0.15 \mathrm{~m}$ and $0.30 \mathrm{~m}$ using $180 \times 120 \times 60$ and $240 \times 160 \times 80$ for a) axial velocity component, b) vertical velocity component, and c) spanwise velocity component; $\mathrm{dt}$ $=0.001 ; U_{\text {inlet }}=1.0 \mathrm{~m} / \mathrm{sec} ; \mathrm{Fr}=7.65$. 140

Figure 7.31 - Square of the turbulence intensity from DREAM ${ }^{\circledR}$ simulations at $y=$ $0.2 \mathrm{~m}$ and $0.40 \mathrm{~m}$ using $180 \times 120 \times 60$ and $240 \times 160 \times 80$ for a) axial velocity component, b) vertical velocity component, and c) spanwise velocity component; $\mathrm{dt}=0.001$; $U_{\text {inlet }}$ $=1.0 \mathrm{~m} / \mathrm{sec} ; \mathrm{Fr}=7.65$. 141

Figure 7.32 - Resolved turbulent kinetic energy at $x=0.15 \mathrm{~m}$ for $180 \times 120 \times 60$ and $240 \times 160 \times 80$ grids; $d t=0.001 ; U_{\text {inlet }}=0.25$ and $1.0 \mathrm{~m} / \mathrm{sec} ; \mathrm{Fr}=1.91$ and 7.65 . 142

Figure 7.33 - Resolved turbulent kinetic energy at $x=0.30 \mathrm{~m}$ for $180 \times 120 \times 60$ and $240 \times 160 \times 80$ grids; $d t=0.001 ; U_{\text {inlet }}=0.25$ and $1.0 \mathrm{~m} / \mathrm{sec} ; \mathrm{Fr}=1.91$ and 7.65 . 142

Figure 7.34 - Resolved turbulent kinetic energy at $y=0.20 \mathrm{~m}$ for $180 \times 120 \times 60$ and $240 \times 160 \times 80$ grids; $d t=0.001 ; U_{\text {inlet }}=0.25$ and $1.0 \mathrm{~m} / \mathrm{sec} ; \mathrm{Fr}=1.91$ and 7.65 . 143

Figure 7.35 - Resolved turbulent kinetic energy at $y=0.40 \mathrm{~m}$ for $180 \times 120 \times 60$ and $240 \times 160 \times 80$ grids; $d t=0.001 ; U_{\text {inlet }}=0.25$ and $1.0 \mathrm{~m} / \mathrm{sec} ; \mathrm{Fr}=1.91$ and 7.65 143

Figure 7.36 - Total averaged energy spectrum at $x=0.15 \mathrm{~m}$ for $180 \times 120 \times 60$ and $240 \times 160 \times 80$ grids; $d t=0.001 ; U_{\text {inlet }}=1.0 \mathrm{~m} / \mathrm{sec} ; \mathrm{Fr}=7.65$ 144 
Figure 7.37 - Total averaged energy spectrum at $x=0.30 \mathrm{~m}$ for $180 \times 120 \times 60$ and $240 \times 160 \times 80$ grids; $d t=0.001 ; U_{\text {inlet }}=1.0 \mathrm{~m} / \mathrm{sec} ; \mathrm{Fr}=7.65$

Figure 7.38 - Total averaged energy spectrum at $y=0.20 \mathrm{~m}$ for $180 \times 120 \times 60$ and $240 \times 160 \times 80$ grids; $\mathrm{dt}=0.001 ; \mathrm{U}_{\text {inlet }}=1.0 \mathrm{~m} / \mathrm{sec} ; \mathrm{Fr}=7.65$.

Figure 7.39 - Total averaged energy spectrum at $y=0.40 \mathrm{~m}$ for $180 \times 120 \times 60$ and $240 \times 160 \times 80$ grids; $d t=0.001 ; U_{\text {inlet }}=1.0 \mathrm{~m} / \mathrm{sec} ; \mathrm{Fr}=7.65$.

Figure 7.40 - Comparison of turbulent kinetic energy from curved and vertical buoyant jets 151

Figure A.3.1 - Results from Hu (2002) code for axial flow (40x40x5) after 10 seconds; pure convection; fully explicit 160

Figure A.3.2 - Results from DREAM ${ }^{\circledR}(40 \times 5 \times 40)$ after 10 seconds; pure convection; fully explicit 161

Figure A.3.3 - Transport of scalar for vertical flow using QSOU3D from Hu (2002)

Figure A.3.4 - Results from transport of scalar quantity using QSOU3D in DREAM $^{\circledR}$ 162

Figure A.3.5 - Results from transport of scalar quantity using Hu (2002) 163

Figure A.3.6 - Results from transport of scalar quantity using QSOU3D in DREAM ${ }^{\circledR}$ 164

Figure A.3.7 - Results from transport of scalar quantity from Hu (2002) after 15 sec 165

Figure A.3.8 - Results from transport of scalar quantity from DREAM ${ }^{\circledR}$ after 15 sec 165

Figure A.4.1 - Initialization of density field for vertical tank filling, $t=0.0$ seconds 169

Figure A.4.2 - Density perturbations during flow for vertical tank filling with constant inlet velocity conditions, $t=\mathbf{5 7 5 . 0}$ seconds 169

Figure A.4.3 - Mass flow rates at the inlet and outlet for first 10 seconds showing conservation of mass throughout the system 170

Figure B.1 - The flow chart of DREAM ${ }^{\circledR}$ for ITM LES . 173 


\section{NOMENCLATURE}
A
a
B
b
$\mathrm{c}_{\mathrm{F}}$
D
F
J
$\mathrm{J} *$
$\mathrm{k}$
K
$1_{\mathrm{ch}}$
$\mathrm{P}$
P'
$\mathrm{Pe}$
Re Reynolds number
$\mathrm{Ri}$
Ro Rossby number
S
$\mathrm{S}_{\mathrm{C}}$
$\mathrm{S}_{\mathrm{P}}$
$\mathrm{t}$
$u$
u
$\wedge$
$u$
$u^{*}$
$\mathrm{u}_{\mathrm{rms}}$
Convection - diffusion coefficient
Coefficient in the discretization equation used in DREAM
Convection - diffusion coefficient
Constant term in the discretization equation
Reciprocal of the turbulent Schmidt number
Hydraulic diameter
Flow rate through a control volume face
Total (convection + diffusion) flux
Normalized flux
Turbulent kinetic energy
Droplet transfer coefficient
Characteristic length scale
Pressure
Pressure correction
Peclet number
Layer Richardson number
General source term
Constant part of the linearized source term
Coefficient of $\Phi_{\mathrm{P}}$ in the linearized source term equation
time
Instantaneous streamwise velocity
Streamwise velocity component
Pseudovelocity in the $\mathrm{x}$ direction used in predictor-correction method
Velocity based on the guessed pressure
Root mean square velocity fluctuations 


$\begin{array}{ll}\mathrm{U}_{\infty} & \text { Terminal velocity of a fuel droplet } \\ \forall_{\text {cell }} & \text { Volume of computational cell } \\ v & \text { Instantaneous vertical velocity } \\ \mathbf{v} & \text { Vertical velocity component } \\ \hat{v} & \text { Pseudovelocity in the y direction used in predictor-correction method } \\ \mathrm{v}^{*} & \text { Vertical velocity based on the guessed pressure } \\ w^{*} & \text { Instantaneous spanwise velocity } \\ \mathbf{w} & \text { Spanwise velocity component } \\ \wedge & \text { Pseudovelocity in the } \mathrm{z} \text { direction used in predictor-correction method } \\ \mathbf{w} & \\ \mathrm{w}^{*} & \text { Spanwise velocity based on the guessed pressure }\end{array}$




\section{GREEK NOMENCLATURE}

$\begin{array}{ll}\alpha & \text { Relaxation factor } \\ \alpha_{\mathrm{p}} & \text { Pressure relaxation factor } \\ \zeta & \text { Vortex length scale } \\ \mu & \text { Viscosity } \\ \delta \mathrm{x} ; \Delta \mathrm{x} & \text { x direction distance between two adjacent points } \\ \delta \mathrm{y} ; \Delta \mathrm{y} & \text { y direction distance between two adjacent points } \\ \delta \mathrm{z} ; \Delta \mathrm{z} & \mathrm{z} \text { direction distance between two adjacent points } \\ \varepsilon & \text { Rate of dissipation of turbulent kinetic energy } \\ \rho & \text { Density } \\ \rho_{m} & \text { Density of the mixture } \\ \tau_{\mathrm{i}} & \text { Interfacial shear stress } \\ \sigma & \text { Surface tension } \\ \eta & \text { Experimental constant } \\ \theta & \text { Liquid-gas contact angle } \\ \cup_{\mathrm{f}} & \text { Diffusion coefficient } \\ \Gamma_{\mathrm{eff}} & \text { Turbulent effective diffusion coefficient } \\ \lambda & \text { Taylor micro scale } \\ \Phi & \text { Arbitrary quantity }\end{array}$




\section{SUBSCRIPTS AND SUPERSCRIPTS}

\section{Subscripts}

B

b

$\mathrm{E}$

e

$\mathrm{N}$

n

$\mathrm{nb}$

$\mathrm{P}$

S

S

$\mathrm{T}$

$\mathrm{t}$

W

W

\section{Superscripts}

1

0

*
Neighbor in the negative $\mathrm{z}$ direction

Control-volume face between $\mathrm{P}$ and B

Neighbor in the positive $\mathrm{x}$ direction

Control-volume face between $\mathrm{P}$ and $\mathrm{E}$

Neighbor in the positive y direction

Control-volume face between $\mathrm{P}$ and $\mathrm{N}$

General neighbor grid point

Central grid point under consideration

Neighbor in the negative y direction

Control-volume face between $\mathrm{P}$ and $\mathrm{S}$

Neighbor in the positive $\mathrm{z}$ direction

Control-volume face between $\mathrm{P}$ and $\mathrm{T}$

Neighbor in the negative $\mathrm{x}$ direction

Control-volume face between $\mathrm{P}$ and $\mathrm{W}$

New value at time $t+\Delta t$ of the dependent variable

Old value at time $t$ of the variable

Previous iteration value of the variable; velocities based on the guessed pressure 


\section{ABBREVIATIONS}

\begin{tabular}{|c|c|}
\hline BFE & buoyant flow event \\
\hline CCD & charged-couple device \\
\hline $\mathrm{CDF}$ & cumulative probability distribution \\
\hline CFD & computational fluid dynamics \\
\hline GB & gigabytes \\
\hline gpm & gallons per minute \\
\hline in & inches \\
\hline $\mathrm{L}$ & liters \\
\hline $\mathrm{mJ}$ & milliJoules \\
\hline $\mathrm{mg}$ & milligrams \\
\hline msec & millisecond \\
\hline msec & nanosecond \\
\hline ITM & Implicit Turbulence Model \\
\hline LES & Large Eddy Simulation \\
\hline fps & frames per second \\
\hline PDF & probability distribution function \\
\hline PIV & particle image velocimetry \\
\hline ppm & parts per million \\
\hline $\mathrm{mm}$ & millimeters $\left(10^{-3}\right.$ meters $)$ \\
\hline$\mu \mathrm{m}$ & micron $\left(10^{-6}\right.$ meters $)$ \\
\hline SGS & Sub grid Scale Model \\
\hline NSEs & Navier-Stokes Equations \\
\hline
\end{tabular}




\section{U.S. CUSTOMARY UNITS AND METRIC EQUIVALENTS}

U.S.

1 in

$1 \mathrm{ft}$

$1 \mathrm{lbm}$ (mass)

$1 \mathrm{fl}-\mathrm{lb}$

1 knot

1 degree

$1 \mathrm{hp}$

$1 \mathrm{lt}$ (long ton)

1 in water $\left(60^{\circ} \mathrm{F}\right)$
METRIC

$25.4 \mathrm{~mm}$

$0.3048 \mathrm{~m}$

$0.4536 \mathrm{~kg}$

$4.4482 \mathrm{~N}$

$0.5144 \mathrm{~m} / \mathrm{sec}$

$0.01745 \mathrm{rad}$

$0.7457 \mathrm{~kW}$

$1016 \mathrm{~kg}$

248.8 Pa (Pascal) 


\subsection{INTRODUCTION}

\subsection{Motivation and Objectives}

Multi-phase flows exist throughout nature, and their behavior is governed by turbulence effects more often than not. A fundamental characteristic of multi-phase flows is the existence of complex interactions between the phases, whereby a range of characteristic lengths scales are present. From the complexity of determining the amount of transfer of mass, momentum, and energy across the interface, modeling of these types of flows is not trivial. As the flow becomes more turbulent, the range of turbulent length scales increases, compounding the problem.

The refueling process in naval destroyer Compensated Fuel Ballast Tanks has a variety of mixing phenomena taking place within the tank as water is expunged as fuel is pumped in. Mixing layers, buoyant jets, and droplet formation and entrainment at the immiscible liquid-liquid interface cause a generation of turbulence and may lead to fuel becoming entrained into the effluent water and pumped overboard, which is an environmental concern. The relative lack of direct knowledge of the behavior of the curved buoyant jet within the Compensated Fuel Ballast Tank has led to this study. For more information of the flow physics during the refueling process within the Compensated Fuel Ballast Tank, the reader is referred to Badeau (2000).

Computational fluid dynamics, CFD, has allowed for modeling of these flows with some success; however, the demands on the available resources are immense using current techniques. As computational resources increase, the ability to model these flows using Large Eddy Simulation (LES) has improved. The objective of LES is to compute the three-dimensional time-dependent details of the relatively large scales of motion, which carry most of the total energy within the flow, while modeling the small scale eddies using some sub-grid scale (SGS) models. Due to the averaging and filter process of the Navier-Stokes equations, traditional LES simulations necessitate the use of some closure model capable of modeling the effects of the small scale structures present in the flow. A high degree of resolution of the domain must be maintained, necessary for acceptable accuracy because SGS models yield the best results when the cut-off wave number lays within the inertial subrange. A variety of SGS models are available, and 
have been applied to a wide range of flows. The reader may refer to Fureby (1999) or Gel (1999) for more details on SGS models.

Unfortunately, traditional LES models have difficulties with complex flow geometries, as well as some difficulties in wall bounded, complex geometries. Often, due to the need for high order accuracy to adequately predict turbulence, many of the SGS models are unstable for certain types of flows due to backscattering of the turbulent kinetic energy (Shi, 2001; Margolin et. al. 2002). Thus, a fairly newly applied approach to LES modeling will be utilized within this study to solve for curved buoyancy driven flows within an enclosure, namely Implicit Turbulence Modeling, ITM, techniques. The cascading effect of turbulent flows is conspiring with scientists to allow this type of numerical technique to be used. As most of the energy is contained within the largest eddies, interactions with smaller eddies, generally no more than an order of magnitude less, allows for this energy to be dissipated at a rate proportional to the resolution of the flow field. Often times, the energy is fully dissipated long before reaching the viscous length scale, even one to two hundred times greater than this length scale. Thus, the smallest scales may not be as crucial to the global behavior of the flow (Margolin and Rider, 2002).

In ITM methods, the Navier-Stokes equations are discretized using a flux conserving, non-oscillatory, control volume technique. These methods not only prevent unphysical oscillations, they also preserve positivity and monotonicity (Oran and Boris, 1993). They are highly stable, and correlate well with the underlying physics of turbulent flows. Therefore, because of the inherent conservative nature of the discretization scheme, these methods have demonstrated the ability to simulate turbulent flows without the need for explicitly using a SGS model. Results clearly show the ability of the ITM methods to predict the inertial subrange of turbulent flows, even for first order upwinding methods, as long as sufficient grid resolution is maintained. Because of the prediction of the inertial subrange, it is evident that ITM methods are capable of dissipating appropriate amounts of turbulent kinetic energy to the smaller unresolved scales (Porter et. al. 1994). Success of ITM methods is from the treatment of each computational cell as a finite volume, as strict flux conservation schemes allow for an accurate 
representation of the coarse-grained equations of motion. ITM methods have compared very well, often times performing better than the SGS models, for a variety of shear dominated flows.

To perform these simulations using ITM methods, a computational code, named DREAM $^{\circledR}$, was developed at West Virginia University under the guidance of Dr. Ismail B. Celik. DREAM ${ }^{\circledR}$ is a fully transient, three dimensional Navier-Stokes solver using accurate second order upwinding discretization schemes, accompanied with a fractional step or projection method. DREAM ${ }^{\circledR}$ is an iterative code by design, while being positive definite, conservative, and computationally efficient. However, DREAM does use a predictor-corrector type method, which accompanied with ITM methods does require small time steps, which hinders the computational efficiency. In order to accurately use ITM methods, high grid resolutions must be maintained. Unfortunately, to adequately resolve the flow field physics, ITM needs a higher grid resolution than many SGS models, as they incorporate important small scale physics at larger grids. Very little research has been performed for application of ITM methods to curved buoyant jet flows, let alone the application to buoyancy driven impingement flows. In this study, an ITM method is used to investigate the behavior of these flows. The curved buoyant jet properties are investigated, as well as the effects of the jet on the global flow field. The behavior of vertical buoyant jet impingement is also investigated using DREAM ${ }^{\circledR}$. The results verify the feasibility of LES and ITM methods for simulation of variable density, turbulent jets, and lend weight to the global strength of this research.

\subsection{Summary}

The objectives of this study are as follows:

1. Validate the ITM methods as implemented into DREAM ${ }^{\circledR}$ for standard turbulent flow phenomenon,

2. Investigate the physics of vertical stably stratified buoyant jets flows,

3. Validate solutions with available experimental data, 
4. Investigate the physics of curved buoyant jets injected into stagnant, liquid in an enclosure,

5. Validate solutions with experimental data. 


\subsection{LITERATURE REVIEW}

\section{Introduction}

The turbulent mixing of a buoyant jet in a density stratified flow is of significant importance in engineering problems. The turbulent shear generated by the plume discharge into the atmosphere results in efficient mixing, and the reduction of the concentration of the pollutants. In stratified fluids, density stratification limits the vertical rise of a buoyant jet, as well as restricting mixing with the surrounding ambient fluid. Although much work has been performed in the study of un-hindered buoyant jets, little research has been done pertaining directly to the impingement of a buoyant jet on a solid surface, and the resulting flow within that region. Simulation of these types of flows is difficult using Large Eddy Simulation (LES) techniques because of computational and numerical demands. To capture the important physics of the flow, most would contend the need in using a sub-grid scale model for closure of the NavierStokes equations and to ensure the appropriate cascading effects observed in turbulent flows. However, this work will use a fairly new technique in LES known as Implicit Turbulence Modeling, or ITM. This study will be one of the first applications of ITM in LES with solid boundary and buoyant flow conditions. As this is a fairly new methodology in LES, most of the available literature is examined in Section 2.1. The literature review of buoyant jets released onto a free surface are briefly summarized in Section 2.2, with this section being used to quantify the momentum dominated region of the buoyant events. Section 2.3 examines the impingement of buoyant jets, released either horizontally or vertically. This section will also be used in the validation of the buoyant jet simulations using DREAM ${ }^{\circledR}$ and ITM methods.

\subsection{Implicit Turbulence Modeling}

The future of CFD codes in turbulence modeling, as computers become faster and faster, is in the numerical technique of Large Eddy Simulation, or LES. A LES is a numerical method, where the largest eddies are computed directly with most simulations resolving the smallest eddies using a sub-grid scale (SGS) model to represent subgrid stresses. The idea behind LES is that the largest eddies that are directly affected by the boundary conditions, account for most flow field energy, and therefore may be computed 
directly. The small-scale turbulence possessed by the smaller eddies is much weaker, contributing less to the Reynolds stresses, and is therefore less critical for many flows. In traditional LES, models are explicitly introduced in the low-pass filtered NavierStokes equations (NSEs) as SGS closures developed in physical space. These methods provide a mechanism by which dissipation of turbulent kinetic energy accumulated at high wave numbers occurs. New research into the field of LES has introduced a new class of LES models, known as Implicit Turbulence Modeling (ITM), where monotonically integrated techniques are used to discretize the Navier-Stokes equations (NSEs), with no pre-filtering being implemented directly. As there are many different numerical control volume algorithms displaying monotonic properties, this section serves the purpose to examine the resulting physics from using ITM type methodologies, and not the resulting mathematical equations. However, Section 3 examines the implementation of these methods into DREAM ${ }^{\circledR}$. For more information on traditional LES methods, accompanied with standard sub-grid scale models, the reader is referred to Gel (1999).

In the numerical solution of the Navier-Stokes Equations, the filtered momentum equation is given by

$$
\frac{\overline{D U}_{j}}{\bar{D} t}=v \frac{\partial^{2} \bar{U}_{j}}{\partial x_{i} \partial x_{i}}-\frac{\partial \tau_{i j}^{r}}{\partial x_{i}}-\frac{1}{\rho} \frac{\partial \bar{p}}{\partial x_{j}}
$$

where the first term on the left hand side is the substantial derivative, the overbars indicate a filtering, and $\mathrm{p}$ is the modified pressure with the gravitational forces included. Various numerical errors are incurred during the discretization of Eq. (2.1.1), with the most important being the spatial truncation error. If Eq. (2.1.1) is modified to include this error, Eq. (2.1.1) becomes

$$
\frac{\overline{D U}_{j}}{\bar{D} t}=v \frac{\partial^{2} \bar{U}_{j}}{\partial x_{i} \partial x_{i}}-\frac{\partial}{\partial x_{i}}\left(\tau_{i j}^{r}+\tau_{i j}^{h}\right)-\frac{1}{\rho} \frac{\partial \bar{p}}{\partial x_{j}}
$$

which is satisfied by the numerical solution. The spatial truncation error appears as an additional stress, which depends on the numerical grid spacing, $h$. If the spatial 
discretization is pth-order accurate, then the spatial stress is of order $\mathrm{h}^{\mathrm{p}}$ (Tamura and Kuwahara (1989)). It is the treatment of this spatial stress numerically where arguably, the largest debate in the field of LES is currently.

The simplest viewpoint is that LES equations should be solved accurately, and that for a given filter width, the grid spacing, $h$, should be chosen sufficiently small to allow the spatial numerical stress to become negligible. The opposite viewpoint is that no explicit filtering should be done and not explicit residual stress model should be used. However, other viewpoints are possible. This means that an appropriate numerical method must be used to solve the Navier-Stokes equations for the mean velocity. Using appropriate grid resolution, which is approximately less than 150 times the viscous length scale, the numerical spatial stresses arise, thus the filtering and residual-stress modeling are performed implicitly by the numerical method. This is why the higher the grid resolution, the more accurate the flow calculations (Okong'o and Knight (1998)). If the appropriate numerical scheme is chosen, such as a control-volume method using accurate positive and monotonic methods, the longer wavelengths contributions to the mean velocity is well resolved. However, implicit turbulence methods are less capable of resolving the shorter wavelength characteristics. The cascading phenomena observed in turbulent flows is also accurately modeled, as the energy is removed from the resolved eddies at a numerical dissipation rate of

$$
\varepsilon_{n u m}=-\tau_{i j}^{h} \bar{S}_{i j}
$$

This allows for the power spectra to observe the $-5 / 3$ slope of the inertial subrange (Knight et. al. (1998)).

In turbulent flows, there is a wide range of scales present throughout the flow, with the largest being the vortical structures, down to the viscous length scale (Kolmogorov length scale). It is a general rule that the larger the turbulent region, the more length scales present within the flow. Oran and Boris (1993) were some of the first to use Implicit Turbulence modeling, ITM, to solve for practical turbulent flows. The authors state that there are three circumstances that allow turbulent problems to be solved 
without resolving all of the length scales in the flow. The first two circumstances are based on the behavior of the spectrum of the turbulent kinetic energy as a function of the flow structures in the system, which determines the wavelength. Oran and Boris (1993) state that as the wavelength decreases, the energy spectrum drops off so quickly that the shortest scale lengths do not contain a large percentage of the total energy within the flow. Therefore, the short length scales are not important dynamically because the cascade of energy from the largest to smallest scales move through intermediary scales. This fact is supported by theory, experiment and computation, where very few features of the flow are important at the viscous length scale, or even scales that are ten to fifty times larger. The last observation by Oran and Boris (1993) is that the nonlinear monotone methods properly connect the flow at the smallest computed scale to the flow at the unresolved scales, which makes the solution reliable down to the smallest resolved scale in the calculation. This means that the higher the grid density, the higher the flow field resolution.

In ITM simulations, the intrinsic high-frequency filters are built directly into the convection discretization, which couples naturally to the resolvable scales of the flow because of the integral control volume and flux limiting techniques used to discretize the NSEs allows for energy to be dissipated through spreading of the eddies over more than one grid cell (Fureby and Grinstein, 1999). This is evident in the analysis of the energy spectrum of the turbulent kinetic energy, where the spectrum drops off at a rate of $\mathrm{E}(\mathrm{k}) \approx$ $[\mathrm{kL}]^{-5 / 3}$ where $\mathrm{k}$ is the turbulent kinetic energy and $\mathrm{L}$ is a characteristic length scale. In this range, the energy extraction from a given scale occurs as a result of interactions between eddies no more than an order of magnitude different in size (Oran and Boris, 1993). Simulations performed by Oran and Boris (1993) for oxygen-hydrogen mixing layers were able to obtain accurate results with resolved scales 25 to 50 times larger than the viscous length scale because most of the energy in the flow is dissipated at longer wavelengths, long before they even reach the viscous length scale.

One of the critical observations of ITM simulations is that the spatial and temporal order of the numerical method is less important than ensuring that the control volume, flux-limiting scheme preserves monotonicity and positivity. Monotonicity 
means that the numerical algorithm does not add unphysical oscillations to the solution. Positivity refers to the particular instance of monotonicity when the quantity is a positivedefinite convected quantity. In fact, work has focused up to this point on second order, upwinding numerical methods. Although there are different numerical schemes to achieve both second order and non-oscillatory effects, one common feature of all ITMs is that they apply to finite volumes of the fluid (Margolin and Rider, 2002). Only finite volume schemes in flux form are considered in this study, because as shown in Section 3.0, DREAM ${ }^{\circledR}$ utilizes this type of scheme. In flux form, the advective terms are the sum of the fluxes entering and leaving a volume, instead of estimating these terms at a single grid point. Conservation of the fluxes ensures the flux into the cell is exactly the negative of the flux leaving its neighbor. Therefore, flux-limiting schemes are naturally conservative to the level of numerical round-off error. Causality, another important property of ITM methods, requires that a fluid being convected from point 1 to point 3 must pass through all cells between them. This results in allowing second order schemes, and in fact, even first order upwinding schemes, to work equally well in ITM simulations as compared to higher order methods, such as fourth order central differencing schemes, as long as the grid resolution is properly posed (Margolin et. al. 2002). These methods work well in mixing problems because they filter out structures smaller than a few grid spacing by spreading out these structures on the grid, thus dissipating them. Therefore, the local, time-dependent dissipation in nonlinear monotone algorithms behave as a subgrid turbulence model for scales smaller than several resolved grid sizes. ITM methods, for certain flow phenomena, properly connect the larger energy containing scales with the unresolved subgrid-scale of motion through this process. Thus, the higher the grid resolution, the smaller the length scales that may be resolved, respectively (Margolin et. al. (2002); Smolarkiewicz and Margolin (1997)). Application of ITM in LES by Margolin et. al. (2002) to determine the decay of turbulence in homogenous incompressible fluids within a triply periodic cube was analyzed, as well as a comparison of the enstrophy results from ITM simulations to pseudo-spectral solutions in DNS. They found that as the viscosity is reduced, enstrophy calculations diverged in the pseudospectral methods; however, up to a certain point in the calculations, there was a high degree of agreement between the two solutions. As the viscous dissipation was 
increased, the pseudo-spectral simulations were solvable, with the results closely agreeing with those of the ITM LES simulations.

For most of the papers reviewed up to this point, application of ITM methods in shear layer simulations was examined. As mentioned previously, there has not been much work in the ITM realm of LES outside of these types of simulations. Breuer (1998) was one of the first to use ITM methods in LES for non-shear flow simulations. Simulations for turbulent flow past a circular cylinder, $\operatorname{Re}=3900$, was studied. However, it is not the physics of the flow past the circular cylinder that is being investigated, but the flow behavior when different SGS and ITM methods were applied. The two SGS models were the standard and dynamic Smagorinsky models. Breuer (1998) mentions that after selecting a mathematical model, the discretization of the Navier-Stokes equations leads to two different types of errors, being the discretization and convergence errors. For ITM type solutions, the ease in the reduction of this error is in the refinement of the grid. This is appropriate for even first order monotonic schemes, which is why Breuer (1998) uses a first order, hybrid type scheme in his analysis to show the inherent success of ITM methods. The second type of error is the convergence error, which again depends on the matrix solver and the convergence criterion. In all simulations, time advancement is performed by a predictor-corrector type scheme. A multi-stage Runge-Kutta method is used in the predictor step, with the Poisson equation being implicitly solved for in the corrector step. An interesting note is that due to the higher stability limit of the Runge-Kutta scheme, an increase of two in computational efficiency was found over use of Adams-Bashforth type schemes. In the description of the ITM scheme, Breuer (1998) states that a more appropriate name for the ITM methods are "LES without a sub-grid scale model." Results of the simulations found that the ITM methods performed very well for three-dimensional simulations, using appropriate grid resolution. However, for two dimensional simulations, it was found that all simulations produced unphysical results, whereby the counter-rotating vortices were not captured, which again lends weight to the ineffectiveness of two-dimensional LES. An important point that Breuer (1998) emphasizes is the fact that all simulations were performed using a $165 \times 165 \times 32$ non-uniform grid, with the discretization schemes used in the Smagorinsky simulations being fourth order. Breuer (1998) concluded that there is a 
small influence of the subgrid-scale model on the back side of the cylinder, where the sub-grid scale models yields a one percent improvement over ITM methods. However, the prediction of the downstream values of $\mathrm{k}$ and skin-friction coefficient using the ITM methods do perform better than the Smagorinsky SGS models by as much as seven percent as compared to the experimental data.

Fureby (1999) used ITM methods to investigate the flow over a backward facing step with a constriction at the outlet for various Reynolds number flows. The traditional LES methods are briefly explained, with the rationale behind the SGS models being presented in the paper. Fureby (1999) explains that monotone methods are capable of handling vorticies in a similar manner as their ability to capturing shocks, making them highly suitable for LES because of the imbedded non-linear filters. Again, the fluxcorrected concept is implemented, which attempts to incorporate the correction term of the convection without violating the physical principals of causality, positivity, and monotonicity. Fureby (1999) uses a linear interpolation scheme, accompanied with a first order upwinding approximation. Time integration is carried out through a CrankNicholson scheme. Results from the ITM type scheme, again, compare well with the available experimental data. Averaged energy spectra at various downstream locations do in fact predict the inertial subrange, lending weight to the use of ITM methods. The re-attachment lengths are predicted well for all simulations, with the ITM methods performing slightly better for the higher Reynolds number cases than the SGS models. Fureby (1999) also states that the predicted probability density of vorticity appears to be largely independent of the SGS model for low-intensity vortical structures, with only a slight influence being observed for the higher-intensity flows. From the results of the backward facing step simulations, Fureby (1999) concludes that with an appropriate grid resolution, ITM methods can correctly channel kinetic energy out of the wave number close to the cutoff wave number, which prevents aliasing. However, an important point to note, one that Fureby (1999) doesn't directly state, is that inclusion of SGS models with coarse grid simulations outperform the ITM simulations. Thus, this only lends to the conclusion that grid resolution is the key to ITM methods. This is due to the coupling of the turbulent viscosity to the grid size used in the simulations in all directions of the flow. 
All reviews thus far have focused on application of ITM to incompressible and subsonic flows. Urbin and Knight (2001) utilize ITM methods in the investigation of a compressible, supersonic boundary layer. Their scheme is second order accurate in both space and time, with the standard monotonicity preserving, control volume formulations being implemented as utilized by Boris et. al. (1992). Again, ITM methods are able to capture the high-frequency energy from the subgrid-scale stresses and heat transfer implicitly through the numerical algorithm. These simulations are compared to the standard Smagorinsky SGS model. One of the difficulties in turbulence modeling, especially in boundary layer flows, is in the determination of the best method of capturing the effects near the wall. To resolve the scales at a wall, the straightforward extension of the classical law of the wall is used. The height of the first cell wall grid is approximately one wall unit. This value is kept constant throughout all grids. Identical grid sizes are used for each of the different SGS and ITM models studies, which are 1.7, 0.32 , and 3.2 million grid cells.

Urbin and Knight (2001) selected the first grid wall unit so as to be comparable with available DNS studies, although the grid spacing near the edge of the boundary layer is substantially larger than would be used in DNS simulations. Results show that the friction and streamwise velocities for the coarsest grid simulations are nearly identical, with the same trend being observed in the comparison of the wall-normal velocity fluctuations. Analysis of the local turbulent viscosity predicted by the Smagorinsky model never exceeds $27 \%$ of the molecular viscosity, which indicates that inclusion of the Smagorinsky model has a negligible effect on the turbulence statistics. Thus, this also lends weight to the fact that ITM methods are capable of capturing turbulence without the need for an explicit SGS for low Reynolds numbers. In the grid refinement studies performed only for the ITM simulations in comparison to the available DNS and experimental data, the ITM simulations again perform very well for all grids considered.

The behavior of the energy spectrum in turbulent flows has been fairly well understood, whereby the largest eddies transfer energy to the smallest ones in a cascading process until being completely dissipated. Thus, for any type of turbulent simulations to be credible, as already mentioned, they must be able to appropriately predict the energy 
spectra in decaying turbulent flows. Porter et. al. (1994) used an ITM type method, in conjunction with a piece-wise parabolic method, to investigate the behavior of the energy spectra in supersonic flows in wakes. Initially, the Mach number was unity, with the initial conditions mean velocity fluctuations being that of a random flow with a prescribed spectrum and characteristic scale. The velocity Fourier spectrum is separated into two components for analysis and characterization, being the solenoidal and compressional modes, with the ratio of the compressional modes to the velocity spectrum being 6.8\%. As these simulations are ITM, the smallest resolvable scales are based on the density of the grid in the wake of the flow, which were 512x512x512 uniformly sized grids. The boundary conditions are periodic in all directions.

Porter et. al. (1994) described the flow based on three temporal regimes, with the time units being those of acoustical time. Initially, for $0<\mathrm{t}<0.3$, shock formations are observed, with $0.3<\mathrm{t}<2.1$ being a supersonic phase and having strong density fluctuations, which develop and maintain themselves. These density fluctuations arise from the shock interactions, where vortex sheets are produced, with the roll up taking place. Porter et. al. (1994) observed that in this phase, the enstrophy reaches its maximum, and being a small-scale quantity, increases with the mesh resolution. This maximum peak is highly dependent on the grid resolution, and varies by $8 \%$ from the coarsest to the finest grid. The last phase is a post-supersonic phase, where $t>2.1$ and the turbulent velocity spectrum exhibits self-similar decay properties, with the velocity spectrum being dominated by the solenoidal component of the spectrum. In this regime, the Taylor wave number reaches its plateau, with the magnitude being dependent on the grid resolution. Further analysis of the energy spectrum showed that during the supersonic phase, compressional modes establish a $\mathrm{k}^{-2}$ velocity power spectrum by $\mathrm{t}=$ 0.5 , and settles to a $\mathrm{k}^{-5 / 3}$ spectrum by $\mathrm{t}=2.0$, with the solenoidal modes building up more slowly. This again shows that the buildup phase is compatible with a Kolmogorov spectrum, and the decay of the energy behaves appropriately.

Fureby and Grinstein (1999) compared various conventional SGS methods to ITM simulations in the studies of forced homogenous isotropic turbulence. The results showed that the simulated energy spectrum depends on the effects of the SGS model only 
toward the high-wave number end of the inertial range and into the viscous subrange. However, results are independent of the SGS model if the resolution is fine enough to ensure that the cutoff wave number lies in the inertial subrange. All of the SGS models performed well in the prediction of the energy spectrum and integral quantities, with improved predictions being obtained with the ITM simulations. Fureby and Grinstein (1999) also state that trying to simulate more complex flows in domains containing walls, ITM simulations have a large advantage over conventional LES SGS methods because there is no explicit filtering in ITM simulations, and thus no commutation error arises, as occurs in the conventional simulations. However, some SGS models do not have an explicit filtering function, which reduces the commutation error as well.

In conclusion, the literature shows the inherent success of ITM methods if proper grid resolution is maintained. Clearly, as ITM simulations are dependent on the grid resolution, coarse grids are less capable of resolving enough of the small eddies to dissipate an appropriate amount of energy out of the flow. This causes not only a lower accuracy in the calculations, but prevents the inertial subrange from being adequately captured to refer to the simulations as LES. Due to the control volume, flux limiting formulation, which ensures conservation of critical properties, ITM may predict turbulence without the explicit use of a SGS model. It is for these reasons that an ITM scheme is utilized in this study, and applied to the NSEs in DREAM ${ }^{\circledR}$.

\subsection{Buoyant Jets}

As buoyant flow events will be extensively studied in this work using ITM computational techniques, basic properties of the jets must be examined. Hwang and Chang (1995) performed numerical simulations of a vertical forced plume in a cross flow of stably stratified fluids. A three-dimensional, time averaged model was employed, which treated the conservation of mass, momentum, and salinity simultaneously. The two-equation standard k- $\varepsilon$ turbulence model was used to simulate the turbulent transport quantities. A constant injection velocity issued vertically into a uniform velocity, cross flow of stably linear stratified fluid was employed. The density of the fluid is computed 
by using Knudsen's formula and the stratification in the environment is caused only by the salinity variation within the system. The numerical computations were performed on a non-uniform and staggered marker in cell grid system. The SIMPLEC algorithm was used to solve the pressure field. Hwang and Chang (1995) described the flow field surrounding the vertical jet in two ways; 1) To surround the fluid, the discharged jet behaves similar to an obstacle placed in front of the flow, where the windward side is the retarding region of high pressure, while the lee side is the low-pressure wake region. Secondly, with respect to the jet flow, the horizontal momentum of the cross flow, the shear layer and the wake entrainment lead to the deflection of the jet in the cross flow direction causing mixing with the surrounding fluid. The surrounding cross flow passes and penetrates through the jet shear layer, resulting in the formation of a pair of vortices on the jet cross-section. The turbulent shear generated by the discharge results in efficient mixing, which rapidly reduces the tracer concentration. In a stably stratified environment, the plume first behaves like a buoyant jet. The initial momentum and the buoyancy of the plume cause the jet flow to move upward, bend over in the cross-stream direction and mix with the heavy bottom fluid. The retardation of the flow development influenced by the stratified environment then leads to the formation of a second and a third pair of vortices above and below the primary vortex pair with a reverse direction. These newly formed vortices grow and suppress the growth of the first set of vortices as the plume flows downstream. As the stratification of the ambient fluid is increased, the retardation of the jet flow from its source efflux becomes more significant, which leads to the alteration of the entrainment mechanism in the stratified cross flow (Hwang and Chang (1995)).

The evolution of uniform, circular, thin shear-layers (jet-like flow) subject to small perturbations were computed using Fourier-spectral discretizations in space and fourthorder predictor-corrector integration in time by Mathew and Basu (1994). The goal of the study was to determine a mechanism of entrainment in circular jets. The flow was incompressible, nominally aligned with the z-axis and periodic with respect to all three Cartesian coordinates. The Reynolds number, based on the initial jet-diameter and the velocity differences across the shear layer, is 1600 and 2400 for cases one and two. It was found that entrainment of the ambient fluid by the jet leads to an increase in the 
region occupied by fluid that is rotational, suggesting that the vorticity exceeds some threshold at this point. In turbulent flows, this is also a region of fluctuating vorticity. Mathew and Basu (1994) states that the mechanism of entrainment is no longer viscous diffusion alone: the jet draws toward itself and ingest irrotational fluid, which then acquires vorticity through the diffusion mechanism. Analysis of the data shows the initial roll-up of the circular shear-layer into four vortex rings per time period. The stages were pairing, instability, growth of the stream-wise structures and the transition to a disordered state. The simulations exhibit the critical features of experimental, spatially developing jets. In the low Reynolds number case, a transition point, which shows the most rapid growth, connects the two stages, which occurs following the breakdown of the rings and the disappearance of the potential core, allowing the stream-wise structures to dominate. In case two, the breakdown occurs much sooner (Mathew and Basu (1994)).

Mathew and Basu (1994) found that four kinds of fluxes across the jet boundary occur, being rotational, irrotational, positive fluxes entering the jet, and the negative fluxes leaving the jet. Larger fluxes occurred at earlier times due to the larger surface areas. Mathew and Basu (1994) determined that the fluxes are all much larger than the differences between each type of flux, suggesting growth and shrinkage during transition from one fluid mechanism to the next. The underlying mechanism is nearly symmetrical and the small differences in the fluxes result in a net growth. Thus, engulfment of the fluid is not the complete picture of turbulent entrainment. The drawing of the fluid packets into the turbulent region by vortical structures must be included, and encircle these structures due to induction, being ingested and expelled several times as it acquires vorticity by viscous diffusion. Thus, it is shown that the entraining flows are strong and clearly associated with vortex rings at early times.

Ideal vortex rings don't mix, but move as blobs through the fluid without exchange with their environment. Jets are known to entrain more effectively when they are turbulent, and entrainment becomes stronger when the geometry of the jet is changed in such a fashion that the generation of individual vortices is enhanced. Auerbach (1991) studied the phenomena of entrainment and ejection in vortex ring flow using an experimental apparatus consisting of a motor driven piston, which displaces a preset volume of water into a glass walled tank. During the vortex roll-up, the growing vortex 
ejects no fluid into its wake and entrainment is the sole mixing process active, thus the quiescent fluid is continuously entrained into the vortex by fluid convection. Auerbach (1991) found that increasing the piston velocity history causes less amounts of entrainment, where as decreasing the piston velocity history leads to an increase in the entrainment. Within the range of $3000<\operatorname{Re}<49000$, entrainment properties of the vortices are not effected, meaning that those generated from the tubes for a fixed length are not effected, whereas those generated at the orifices are. When the piston stops moving and the roll-up phase ends, the convective entrainment stops abruptly, and the vortex rings appears to be self-similar, which is also independent of the Reynolds number. During the laminar and wavy phases, the reduced entrainment is now predominantly diffusive and takes place continuously. A further abrupt jump takes place in the mixing properties of rings when they become turbulent, i.e. playing a vital role in the nature and duration of this phase. Entrainment remains of an essentially continuous diffusive nature. The volume of fluid ejected by the ring was found to be dependent on the elapsed time of turbulent ring motion and the volume of each of these hairpins (Auerbach (1991)).

The flow and density distribution produced by injecting dense fluid upwards at the bottom of a homogenous fluid were investigated experimentally and theoretically by Baines et. al. (1990). Salt water was injected into a tank of fresh water, with both axisymmetric and line sources being studied using a small scale experiment. The experiments performed by Baines et. al. (1990) are very similar to those performed by Friedman et. al. (1999). As in the Friedman simulations, the turbulent fountain formed rises to a maximum height, which was related to the inflow Froude number, and then falls back and spreads. If the inflow is continued, the box begins to fill yielding a stable stratified environment. The evolution is determined by the rate of entrainment into the fountain from the ambient fluid. Re-entrainment of fluid into the fountain continually changes the density profile in the mixed fluid collecting at the bottom of the chamber. The top of the fountain rises linearly in time, at a rate which, for axisymmetric fountains, has been shown both experimentally and theoretically to be half the rate of rise of the free surface due to the inflow. Baines et. al. (1991) concluded that once the mixed fluid at the bottom of the chamber has risen above the fountain, its density profile remains constant. 
For line fountains, the results are less precise. This is most likely due to the instability within the jet, which causes the flow to switch irregularly from a symmetrical state to one in which the downflow occurs on one side only, and with a smaller maximum height. The experimental values corresponded well with the theoretically calculations based on an inlet densimetric Froude number.

Experiments and simulations have found that the ability of a turbulent plume to spread is highly dependent on the amount of ambient fluid that may be entrainment within the jet. Turner (1986) investigates the entrainment assumption by relating the inflow velocity to the local mean velocity of a turbulent flow. The entrainment assumption, as stated by Turner (1986) is "the mean inflow velocity across the edge of a turbulent flow is assumed to be proportional to a characteristic velocity, usually the local time averaged maximum mean velocity or the mean velocity over the cross-section at the level of inflow." Turner (1986) successfully applied the entrainment assumption to describe a variety of natural phenomena over a wide range of turbulence length scales. The first application was to plumes rising in stably stratified surroundings, and it was later extended to inclined plumes and other buoyancy driven problems. Turner (1986) explicitly states that these buoyant forces inhibit mixing across a density interface. Another important contribution by Turner (1986) is in the investigation of the behavior of the plume with very different physical properties, such as viscosity differences. Turner (1986) found that when the viscosities are comparable, there is appreciable turbulent entrainment; however, for larger variations, it may become completely hindered with no entrainment taking place.

\subsection{Impingement of Buoyant Jets on a Non-Free Surface}

The unobstructed vertical turbulent buoyant jet has been studied extensively theoretically and experimentally. The behavior of the jet downstream of the inlet is known; however, in the region near the jet impact zone, little research has been performed, as experimental quantification is difficult.

An experimental study was performed by Noutsopoulos et. al. (1979), which investigated the effects of a round, vertical, turbulent, buoyant jet of diameter d, impinging on a horizontal concentric circular solid disk. As previous studies have 
shown, turbulent mixing causes considerable dilution, which may reduce pollution in a variety of environmental flows. The buoyant flow was generated using a water jet, released vertically into an ambient salt-water solution. The densimetric Froude number is used to characterize the flow, expressed as

$$
F r=\frac{\rho_{0} V_{0}^{2}}{g\left(\rho_{a}-\rho_{0}\right) d}
$$

where $\rho_{o}$ and $V_{o}$ are the inlet density and velocity of the water, $g$ is the gravitational constant, $d$ is the diameter of the inlet (hydraulic diameter may be used for non-circular jets), and $\rho_{\mathrm{a}}$ is the ambient density of the salt water. Noutsopoulos et. al. (1979) divided the overall flow field into three distinct regions, being; A) Flow field a distance dt before the disk, where no effects of the plate are felt, B) The flow field a distance dt to the disk, were the flow field is highly effected by the plate, and C) The flow field after impacting the disk. The experiment was designed to allow for the plate to move to help in determining densimetric densimetric Froude number dependence on the downstream plume resulting from an impact. Results from experiments found that at a certain distance before the impact region, which depends on the densimetric densimetric Froude number and distance between the jet and the impact plate, the jet develops as though it were unobstructed. The region after the buoyant jet impacts the flow and continues behaves like a plume, which is dominated by buoyancy. No results are presented for the region just before impaction, as like most experimental studies, it is difficult to obtain measurements within this region (Noutsopoulos et. al. (1979)). A comparison of homogenous non-buoyant jets to the buoyant jet results indicates a wider spreading before the impact region of the buoyant jet. This allowed Noutsopoulos et. al. (1979) to conclude that as spreading is a measure of a turbulence scale, then larger turbulence length scales are present in the buoyant jet.

A submerged water discharge into a cold and stratified body of water is often encountered in engineering and environmental flows. $\mathrm{Gu}$ (1998) investigated the behavior of a two-dimensional buoyant jet resulting from temperature gradients. An example of this flow is when submerged warm water discharges into a stratified lake or reservoir with an ice cover. The goal of this study was to determine the large-scale convective re-circulation and flow processes in a cold body of water, induced by the 
resulting buoyant jet. The two-dimensional simulation consisted of a horizontal buoyant jet, injected at a given flow rate and temperature, into an ambient fluid with a different temperature. This allowed for the study of both the standard buoyant jet, along with a negative buoyant jet depending on the inlet temperature of the water. The simulation times needed for buoyant jet boundary impingement are presented for different temperatures. The standard k- $\varepsilon$ epsilon turbulence model was used in the simulations, where variations in the transverse direction were in fact ignored. Gu (1998) specified the ice cover on the upper boundary using a liquid wall boundary condition for the velocities, accompanied with a temperature specification. An outlet boundary condition was also specified. The flow was studied for a variety of densimetric densimetric Froude and Reynolds numbers. $\mathrm{Gu}$ (1998) found that re-circulation due to jet entrainment and ambient water replenishment in the regions above and below the jet create a vortex on each side of the jet. Dilution of the resulting jet is highly sensitive to the temperature gradient of the flow, which may cause the jet, after enough dilution, to become a negative buoyant jet ( $\mathrm{Gu}(1998))$.

Through experimentation by many researchers, it has been shown that a threedimensional vertical wall-jet has a large rate of spread in the direction parallel to a wall, and a small rate in the direction perpendicular to it. The turbulent zone is much wider in the direction parallel to the wall than perpendicular to it as well (Baines (1985)). Experiments performed by Baines (1985) determined the wall effects on entrainment and jet behavior in a negative, one-percent density difference, buoyant, vertical forced plume flowing parallel to the wall. The entrainment rate is different for a jet along a wall compared to a free jet due to the cross-sectional differences between the two types of flows. The entrainment rate should increase as the peripheral length increases; however due to the wall effects on the turbulent flow field, the entrainment rates have actually been shown to decrease. Baines (1985) compared the volume flux between the free jet and wall bounded jet and concluded that close to the source, the wall bounded jet has a higher rate of entrainment near the wall, and farther from the wall, the effects reduce. This allowed for two conclusions namely; 1) the distortion of the cross-section gives a larger surface over which entrainment may occur, and 2) the wall proximity has changed the turbulent field enough as to alter the driving mechanism of entrainment. 
The characteristics of plane self-preserving buoyant turbulent adiabatic wall plumes were examined experimentally by Sangras et. al. 2000). Measurements of the velocity properties of the adiabatic wall plume were described, with emphasis being placed on conditions of the jet far from the source, where self-preserving behavior is approximated. The experiments used helium as the buoyant jet, which was allowed to rise along a smooth, plane, vertical wall. A porous material was used on the top of the experimental apparatus, and measurements were not taken in this region. Laser-induced fluorescence (LIF) was used to measure properties of the velocity field. A density ratio of 0.75 was used in the experiments, with Reynolds and densimetric densimetric Froude numbers being 740 and 3.50, respectively. The densimetric Froude number is actually the source densimetric Froude number, which was based on the density difference, and is used to approximate the densimetric Froude numbers far downstream of the source as well. The buoyant jet was observed to become self-preserving approximately $92-156$ source widths from the source. This yielded larger near-wall mean velocities than observations within the flow development region near the source. Close to the wall, the behavior of the buoyant jet was similar to velocity fluctuation intensities in non-buoyant jet experiments. The power spectra obtained from the velocity fluctuations exhibit the well known $-5 / 3$ power inertial-convective decay region, but the measurements were unable to reproduce the -3 power inertial diffusive decay region that is generally observed in buoyant turbulent flows (Sangras et. al. 2000)).

Buoyant jet flows have been extensively studied by fire investigators in the hope of determining the heat transfer to the walls from a fire driven plume. As the air becomes heated and rises, especially if a point source is used, a buoyant jet ensues. This jet behaves in a similar manner to liquid-liquid buoyant jets. Cooper (1989) performed experiments with the goal of determining the total heat transfer to walls during fires with buoyant plumes. Estimates for the mass, momentum, and enthalpy flux were obtained immediately upstream of the ceiling-wall junction. Results indicated that depending on the proximity of the plume to the wall, energy releases were found to be in the range of $200-2000 \mathrm{~kW}$ (Cooper (1989)).

An experimental study dealing with the impact of a turbulent, 2-D plane, nominal buoyant jet with a submerged solid boundary was studied by Cavalletti and Davies 
(2003). The experimental data demonstrated that the impact of a turbulent plane buoyant jet causes a complex disturbance field to be generated near the site of impingement. The principal features of the disturbance field, i.e. rebound vortices, out flowing current, etc., are qualitatively self-similar. This fact is regardless of flow incidence angle with respect to the wall. The quantitative effects of impingement upon the structure of the velocity and concentration fields within the approaching buoyant jet are manifested primarily in (1) a distortion of the free buoyant jet decay in centerline velocity, and (2) a distortion in the concentration profiles associated with the far-field buoyant jet flow. The placement of the solid boundary was at a distance so as to not to allow for the development of a plume. The authors note that these types of cases require much more study, and only indirect conclusions have been made thus far, whereby experimental data has been extended (Cavalletti and Davies (2003)).

Flow and heat transfer to a circular cylinder with a hot impinging air jet was studied by Kang and Greif (1992). The effects of the Reynolds and Grashof numbers on the flow and heat transfer, as well as those of the wall temperature, the jet width, and the distance between the nozzle and the cylinder were investigated. The authors also present correlation curves with experimental data. The results presented in the paper were stable and symmetric over all dimensionless parameters used to describe the flow. The interaction of the buoyant impinging jet with the cylinder, as well as with the surroundings makes the flow and heat transfer to the cylinder very different from that of uniform flow (Kang and Greif (1992)). The authors found that the effects of buoyancy, diffusion, and cooling of the jet ahead of the cylinder, the development of a wall jet, and the re-circulating bubble all affect the flow and heat transfer. The width of the inlet jet, as well as the distance between the jet and the cylinder also had a strong effect on the heat transfer to the cylinder, which is what was expected. It was concluded that the average Nusselt number increases with increasing Reynolds and Grashof numbers and narrow banded correlation curves are obtained by introducing an effective Reynolds number.

Friedman et. al. (2001) performed experiments dealing with droplet formation and the prediction of size distributions from an immiscible interface impinged with a vertical, negatively buoyant jet. Experiments showed that when an upward-flowing water jet 
impinges on an interface with an immiscible layer of lighter oil above it, the jet momentum lifts the interface and forms a cavity. Below a threshold based on the jet Richardson and Reynolds numbers, no droplets will form; however, above this threshold, oil drops are formed by two mechanisms. At high Richardson numbers, an oil lip created at the edge of the cavity detaches to form oil droplets in the water below, whereas at a low Richardson number, the water cavity becomes unstable and alternately collapses and reforms. As the water impinges on the solid surface, a negative buoyant force drags fuel entrained into the water downward, which then break and form droplets. The collapsing cavity impacts the interface, it drags down fingers of the lighter phase, which then breaks and form oil droplets. Experimental observations suggest a log normal distribution of droplet sizes, with mode diameters ranging from 0.6 to $1.5 \mathrm{~mm}$. Characteristic diameters decrease primarily with increasing Reynolds number, Richardson number, and to a lesser extent with decreasing viscosity ratio (Friedman et. al. (2001)). The results from this paper have been used in the verification and quantification of the performance of buoyant jets using ITM LES methods as implemented into DREAM ${ }^{\circledR}$, and further discussion is deferred to Chapter 6.0.

Shy (1995) studied mixing processes involving organized large-scale and chaotic small-scale motions across a sharp density interface using a $\mathrm{pH}$-sensitive, laser induced fluorescence technique in a water tank. This non-intrusive technique allows one to distinguish fluid that has been molecularly mixed from that which has been merely stirred. A turbulent round jet impinged from above on the sharp density interface over a flow Reynolds numbers between $2500 \leq \operatorname{Re} \leq 25,000$ and flow Richardson numbers ranging from $0 \leq \mathrm{Ri} \leq 5$, each based on the local jet scales at the interface. It was found that at large Reynolds numbers, molecular mixing first occurs at the perimeter of the jet front, forming a mixed layer, in contrast to a jet in a uniform environment, where engulfment occurs in the back of the large vortical structures. For relatively weak stratification approaching the atmospherically relevant situations, as the jet penetrates into the density interface and continues to advance, the mixed layer develops into a complex reverse jet that ejects backward along the sides of the original jet core. Surprisingly, the latter remains little mixed. Shy (1995) reasoned that this was the explanation to why the undiluted cloud base air has been found at all levels within 
cumulus clouds during aircraft penetration. At moderate stratification, a mixing transition was observed across which the mixed layer thickness changed by an order of magnitude, showing that the Reynolds number also plays a role in mixing in stratified flows. With stronger stratification, the jet front barely penetrates the interface. A physical model was presented by Shy (1995) in order to explain the jet transport and the mixing transition across the density interface. Shy (1995) concluded from this physical model that entrainment at the density stratified interface is not solely dependent on the Richardson number, but also depends on the Reynolds number. 


\subsection{IMPLICIT TURBULENCE MODELING}

\subsection{LES with Implicit Turbulence Modeling Techniques}

This chapter explains the numerical development of DREAM $^{\circledR}$, a CFD code developed at West Virginia University (Celik and Badeau, 2003), including the justification for use of implicit turbulent modeling (ITM). Developing a new CFD code is a tedious, difficult, and constantly ongoing process. The control volume discretization techniques as applied to $\operatorname{DREAM}^{\circledR}$ in its soundest and most fundamental form are explained, as well as gridding, coupling effects, and other important features inherent to the workability of the solver.

DREAM $^{\circledR}$ was developed to be a fully transient, three dimensional Navier-Stokes solver using accurate second order, upwind discretization schemes, accompanied with a fractional time step or projection method. DREAM ${ }^{\circledR}$ was an iterative scheme by design, while being positive definite and conservative. It is these properties of the method that allows for ITM to be used in the LES simulations without an explicit sub-grid scale model. Validation of DREAM ${ }^{\circledR}$ is elucidated by Celik and Badeau (2003), with more validation cases presented in Chapter 4. Simplicity is the key to this code, where everything is kept to its most basic form, such as Cartesian coordinates, staggered, uniform or non-uniform grids etc. The equations solved for are put in a general form given by

$$
\frac{d \Phi}{d t}=F(\Phi)+S
$$

where $\mathrm{F}$ is the net flux per unit mass through the surface of a control volume, and $\mathrm{S}$ is the source term including the pressure gradient. All equations take the generalized form as given by Eq. (3.1.1).

Integration of Eq. (3.1.1) is the beginning of the differences between the traditional LES methods as compared to ITM methods. In practical LES implementation, the prefiltering of Eq. (3.1.1) really has no explicit effect on the variables that are solved, as the filtering is performed when deriving the LES equations, which then are discretized, although the filtering does have an indirect effect on producing unresolved transport 
terms that required additional closure modeling. Therefore, cell averaging may be treated as a built in filtering process, which is similar to a square, top-hat, filtering function (Fureby and Grinstein, 1999).

\subsection{Discretized Equations in DREAM ${ }^{\circledR}$}

In this section the derivation is given for the discretized equations for a generic transport variable. These equations are applicable to any of the primitive flow variables, such as volume fraction, density, temperature, or velocity, etc. As volume fraction, density, and temperature are never negative quantities, it is important that the numerical scheme used does not produce un-physical quantities, which is why positivity is so important in using ITM methods, but not required.

The generalized transport equation for a generic field variable $\phi$ is

$$
\frac{\partial(\rho \phi)}{\partial t}+\frac{\partial}{\partial x_{j}}\left(\rho u_{j} \phi-\Gamma \frac{\partial \phi}{\partial x_{j}}\right)=S_{\phi}
$$

where repeated $\mathrm{j}$ index implies a summation, $\rho$ is the density, $\Gamma$ is the diffusion coefficient, and $\mathrm{u}$ is the velocity. It is important to note that Eq. (3.2.1) applies to single fluid or single component flows only; however, for many of the simulations presented for buoyant jet cases, where there is a mixture between two fluids the following substitution can be applied;

$$
\rho \Rightarrow \varepsilon_{k} \rho_{k} \text { and } \Gamma \Rightarrow \varepsilon_{k} \Gamma_{k}=\rho_{k} \varepsilon_{k} \Gamma_{k}^{*}
$$

where the subscript " $\mathrm{k}$ " represents the property of the $\mathrm{k}^{\text {th }}$ component, and $\varepsilon$ denotes the volume fraction. If the density is treated as a mixture density, then only one set of conservation equations needs to be solved for. The volume fraction is computed using another scalar transport equation, ranging from 0 to 1 , which then allows for the mixture density to be calculated for the entire domain, given by

$$
\rho_{m}=\left(1-\varepsilon_{1}\right) \rho_{1}+\varepsilon_{1} \rho_{2}
$$

The volume fraction can also be treated as a porosity for a liquid-solid or gas-solid mixture. To account for the slip-velocity between the two phases, a pressure based three dimensional slip velocity relationship, as shown in Kandil (2001), is utilized using a 
constant droplet size of $2.5 \mathrm{~mm}$. This droplet diameter corresponds to the experimental observations by Friedman et. al. (2001). This slip velocity is added to the mixture momentum equation as a source term. This model is not explained further here as it was only implemented into DREAM ${ }^{\circledR}$, and for details on this model, the reader is referred to the Kandil's (2001) study. Note that the phase density can be zero when the volume is zero, and this must be handled separately. For example, the equation of continuity for the $\mathrm{k}^{\text {th }}$ component is written as (no summation over repeated $\mathrm{k}$ index)

$$
\frac{\partial\left(\varepsilon_{k} \rho_{k}\right)}{\partial t}+\frac{\partial}{\partial x_{j}}\left(\varepsilon_{k} \rho_{k} u_{j}\right)=S_{m}
$$

where $S_{m}$ denotes any possible mass interchange among the components or phases with substitutions of the kind given by Eq. (3.2.2). The continuity equation is given by

$$
\frac{\partial(\rho)}{\partial t}+\frac{\partial}{\partial x_{j}}\left(\rho u_{j}\right)=S_{m}
$$

To simplify the derivation process, only one-dimension is considered, i.e. the $\mathrm{x}$-direction, and thus re-writing Eqs. (3.2.4) and (3.2.1) as

$$
\begin{gathered}
\frac{\partial(\rho \phi)}{\partial t}+\frac{\partial}{\partial x}\left(\rho u \phi-\Gamma \frac{\partial \phi}{\partial x}\right)=S_{\phi} \\
\frac{\partial(\rho)}{\partial t}+\frac{\partial}{\partial x}(\rho u)=S_{m}
\end{gathered}
$$

For definition and simplification purposes, two new variables $J_{\mathrm{x}}$, the total flux, and $\mathrm{F}_{\mathrm{x}}$, the convective flux, are defined as

$$
\begin{gathered}
J_{x}=F_{x} \phi-\Gamma \frac{\partial \phi}{\partial x} \\
F_{x}=\rho u
\end{gathered}
$$

Hence, Eqs. (3.2.5) and (3.2.6) become

$$
\begin{aligned}
& \frac{\partial(\rho \phi)}{\partial t}+\frac{\partial}{\partial x}\left(J_{x}\right)=S_{\phi} \\
& \frac{\partial(\rho)}{\partial t}+\frac{\partial}{\partial x}\left(F_{x}\right)=S_{m}
\end{aligned}
$$


Performing some algebra, the reasons for which will become clear later, multiplying Eq. (3.2.10) by $\phi$, and then subtracting it from Eq. (3.2.9), yields

$$
\frac{\rho \partial(\phi)}{\partial t}+\frac{\partial}{\partial x}\left(J_{x}\right)-\phi \frac{\partial}{\partial x}\left(F_{x}\right)=S_{\phi}-\phi S_{m}
$$

Eq. (3.2.11) will be used as the working equation, and integrating it over a control volume using the usual assumptions employed in the so-called control volume approach (Patankar (1980)), results in

$$
\rho_{P} \Delta \forall \frac{d \phi_{p}}{d t}+J_{e}-J_{w}-\phi_{P}\left(F_{e}-F_{w}\right)=\bar{S}_{\phi} \Delta V-\phi_{P} \bar{S}_{m} \Delta V
$$

where the indexes P, e, and $\mathrm{w}$ are as shown in Figure 3.2.1.

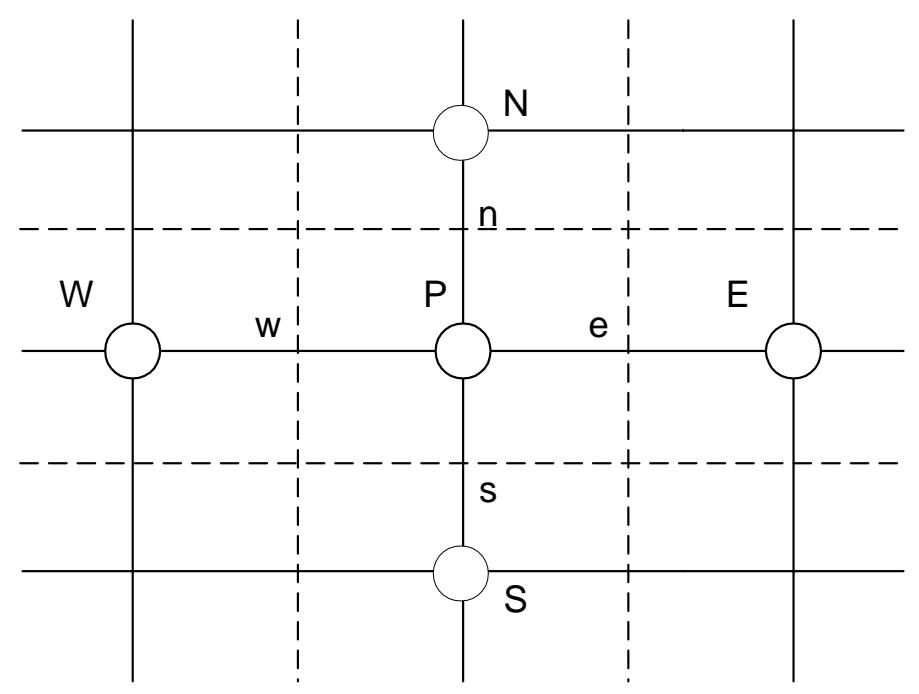

\section{Figure 3.2.1 - Grid used for integration of transport equations}

In Eq. (3.2.12), the over bar, “-“, indicates a volume averaged quantity. The expressions for $\mathrm{J}_{\mathrm{e}}, \mathrm{J}_{\mathrm{w}}, \mathrm{F}_{\mathrm{e}}$, and $\mathrm{F}_{\mathrm{w}}$, and another variable $\mathrm{D}$, the diffusion flux, are given by

$$
\begin{array}{llll}
D_{e} & =\left(\Gamma A \frac{d \phi}{d x}\right)_{e} & \text { and } & D_{w}=\left(\Gamma A \frac{d \phi}{d x}\right)_{w} \\
F_{e} & =(\rho u A)_{e} & \text { and } & F_{w}=(\rho u A)_{w} \\
J_{e}=F_{e}-D_{e} & \text { and } & J_{w} & =F_{w}-D_{w}
\end{array}
$$

where A denotes the cell face areas. The source term is linearized in the usual manner, giving 


$$
\bar{s}_{p}=\phi_{p} S_{p}+s_{c}
$$

It is important to note that the source term linearization should be selected such that $\mathrm{s}_{\mathrm{p}}<$ 0 , always. Also, the treatment of the source terms allows for the inclusion of baffles and walls within the domain. For more on this type of treatment, the reader is referred to Appendix B.2. Moving all non-derivative terms in Eq. (3.2.12) to the right hand side and re-writing yields

$$
\begin{gathered}
f_{\text {rhs }}=-\left[\begin{array}{l}
\left(J_{e}-\phi_{P} F_{e}\right)-\left(J_{w}-\phi_{P} F_{w}\right)+ \\
\left(s_{p}-s_{m}\right) \Delta \forall \phi_{P}+s_{c} \Delta \forall
\end{array}\right] \\
\rho \Delta \forall \frac{d \phi_{P}}{d t}=f_{r h s}
\end{gathered}
$$

Now, the time discretization of Eq. (3.2.16) can proceed in many ways. It is desirable to have the options of (i) fully implicit, (ii) fully explicit, (iii) Crank-Nicholson, etc. It is also desirable to have the option of making only the diffusion terms explicit and handling the convection terms implicitly. The spatial discretization can also be changed by simply changing the method for evaluation of the cell face values in the fluxes, $J_{e}$ and $F_{e}$, etc. Analysis of Eqs. (3.2.15) and (3.2.16) is the critical component in ITM because in the application of flux limiting methods, it is important to choose a higher-order convective flux function that works well in smooth regions and a lower order flux function that works well near discontinuities, such as upwind differencing. The hybridization of these functions into a single function will then lead to a proper unconditional monotone method, with a high enough order to ensure that the numerical diffusion doesn't hinder the results, such as second order methods.

Therefore, we shall employ a generalized scheme as follows:

$$
\rho \Delta \forall \frac{d \phi_{P}}{d t}=\alpha f_{r h s}+\left(\alpha_{d}-\alpha\right) f_{d}+(1-\alpha) f_{r h s}^{o}+\left(1-\alpha_{d}\right) f_{d}^{o}
$$

where $f_{d}=D_{e}-D_{w}, \alpha$ is the implicitness factor, $\alpha_{d}$ is the implicitness factor for the diffusion, $-\alpha f_{d}$ cancels the diffusion included already in the right hand side of Eq. (3.2.15), and the other two terms constitute the implicit and explicit part of the diffusion term. Now what remains is the spatial discretization scheme for evaluation of the $f_{\text {rhs }}$ and 
$f_{d}$. This is done following the generalized scheme described by Patankar (1980), thus giving

$$
J_{e}-F_{e} \phi_{P}=a_{E}\left(\phi_{P}-\phi_{E}\right) \quad \text { and } J_{w}-F_{w} \phi_{P}=a_{W}\left(\phi_{W}-\phi_{P}\right)
$$

The diffusion terms may be accurately represented by central differentiation, i.e.

$$
\begin{gathered}
D_{e}=\left(\Gamma A \frac{d \phi}{d x}\right)_{e}=d_{E}\left(\phi_{E}-\phi_{P}\right) \\
D_{w}=\left(\Gamma A \frac{d \phi}{d x}\right)_{w}=d_{W}\left(\phi_{P}-\phi_{W}\right) \\
d_{E}=\left(\frac{\Gamma A}{\Delta x}\right)_{e} \quad \text { and } \quad d_{W}=\left(\frac{\Gamma A}{\Delta x}\right)_{w}
\end{gathered}
$$

Thus,

$$
\begin{gathered}
f_{r h s}=\left\{\begin{array}{l}
-\left(a_{E}+a_{W}\right) \phi_{P}+a_{E} \phi_{E}+a_{W} \phi_{W}+ \\
\left(s_{p}-s_{m}\right) \Delta \forall \phi_{P}+s_{c} \Delta \forall
\end{array}\right\} \\
f_{d}=-\left(d_{E}+d_{W}\right) \phi_{P}+d_{e} \phi_{E}+d_{w} \phi_{W}
\end{gathered}
$$

Here, the coefficients $a_{E}$ and $a_{W}$ are calculated from the relations given by Patankar (1980), which are:

$$
\begin{gathered}
a_{E}=D_{e} A\left(\left|P_{e}\right|\right)+\max \left(-F_{e}, 0\right) \\
a_{W}=D_{w} A\left(\left|P_{w}\right|\right)+\max \left(F_{w}, 0\right) \\
P_{e}=\frac{F_{e}}{D_{e}} \text { and } P_{w}=\frac{F_{w}}{D_{w}}
\end{gathered}
$$

The appropriate choice for ITM simulations is the power law scheme for determining $\mathrm{A}\left(\operatorname{abs}\left(\mathrm{P}_{\mathrm{e}}\right)\right)$, which has shown to be second order as it toggles between upwind differencing and central differencing schemes depending on the value of the Peclet number. However, in LES simulations, the Peclet number is generally smaller than 2.0, which ensures that the central differencing scheme will be generally utilized, which is second order in nature. Also, this indicates that the leading truncation error of the scheme is now proportional to the grid spacing. This lends weight to the statement that the grid resolution behaves as a SGS model (Brewer, 1998), in which Brewer (1998) successfully 
used a hybrid function in the numerical simulations, which is first order accurate. The power law scheme is defined as

$$
A\left(\left|P_{e}\right|\right)=\max \left(0.0,1.0-0.1|P e|^{5}\right)
$$

Therefore, utilizing this type of formulation, an ITM type formulation is used in DREAM $^{\circledR}$ which is second order accurate in space, conservative, and monotonic. Equation (3.2.24) clearly exhibits positive definite properties, as the coefficients are not allow to be negative. The examination of the temporal discretization scheme is described in the following section.

\subsection{Fractional Step (Projection) Method}

The fractional step method, also known as the projection method, is the fundamental numerical method used for solving the incompressible Navier-Stokes equations. In this document, the terminology of fractional step and projection method will be used interchangeably. Generally speaking, the major difficulty in obtaining time-accurate solutions for incompressible flows arises from the fact that the continuity equation doesn't contain pressure and it's time derivative explicitly. The constraint of mass conservation is achieved by an implicit coupling between the continuity equation and the pressure term within the momentum equation. Handling this type of flow may be difficult, especially when trying to maintain stability; thus the projection method is used, whereby the projection step is calculated through subtracting the pressure contribution to find starred velocity field, then a Poisson equation is solved to find the new pressure field. 


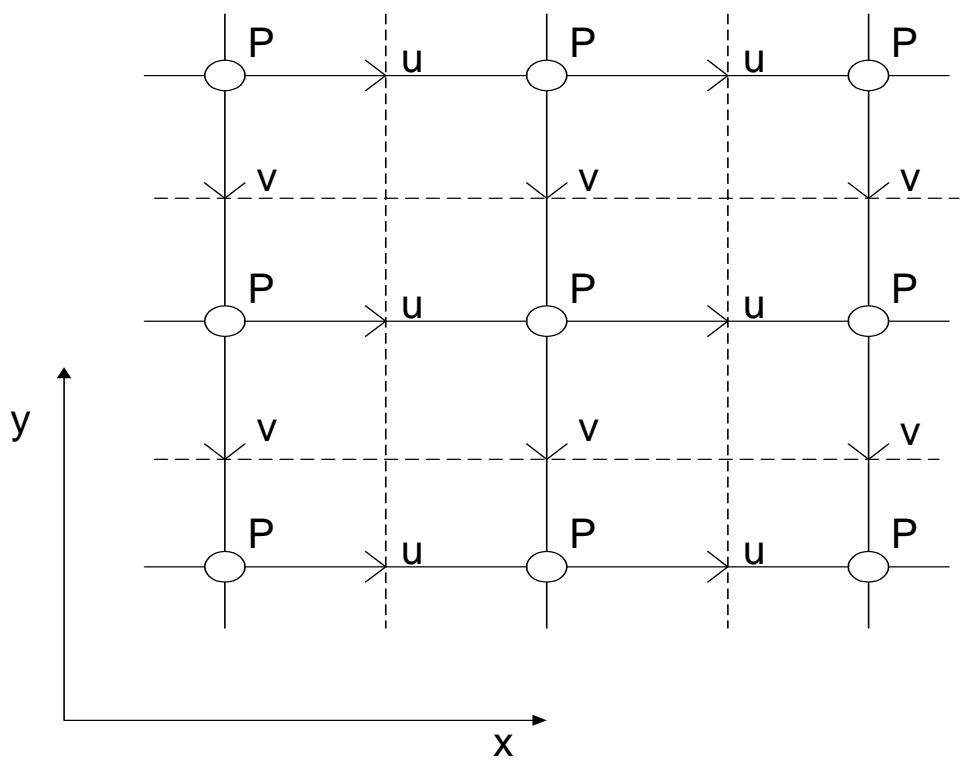

Figure 3.3.1 - Staggered grid used in the DREAM ${ }^{\circledR}$ code

Generally speaking, there are a variety of methodologies in which the projection method, or fractional step method may be implemented into DREAM $^{\circledR}$. The discretized momentum equations are represented by the following functional relationships;

$$
\rho^{n}\left(\frac{u^{n+1}-u^{n}}{\Delta t}\right)=\alpha f^{n}+\beta{\underset{\sim}{f}}_{\sim}^{n+1} P^{n+1}
$$

Since the pressure at the $n+1$ time level is not known, we must approximate Eq. (3.3.1) as

$$
\rho^{n}\left(\frac{u^{*}-u^{n}}{\Delta t}\right)=\alpha f_{\square}^{n}+\beta \underset{\sim}{f^{*}}-\underset{\sim}{\nabla} P^{n}
$$

It is assumed that the velocity field in $\mathrm{u}^{\mathrm{n}}$ and the pressure field $\mathrm{P}^{\mathrm{n}}$ satisfy the continuity equation, but $\mathrm{u}^{*}$ calculated from Eq. (3.3.2) does not necessarily satisfy the same continuity equation. If Eq. (3.3.2) is subtracted from Eq. (3.3.1) then

$$
\rho^{n}\left(\frac{u^{n+1}-u^{*}}{\Delta t}\right)=\beta\left({\underset{\sim}{f}}^{n+1}-f_{\sim}^{*}\right)-\underset{\sim}{\nabla}\left(P^{n+1}-P^{n}\right)
$$

At this point in the derivation, it is assumed that the difference between $\mathrm{f}^{\mathrm{n}+1}$ and $\mathrm{f}^{*}$ is small. This constitutes the change in $\mathrm{u}^{*}$ if $\mathrm{f}^{\mathrm{n}+1}$ were to be used in Eq. (3.3.2) instead of $\mathrm{f}^{*}$ $=\mathrm{f}\left(\mathrm{u}^{*}\right)$. Usually, the implicit part of Eq. (3.3.1) includes the difference term, hence neglecting the term $\mathrm{f}^{\mathrm{n}+1}-\mathrm{f}^{*}$ amounts to the error in treating the diffusion terms semi- 
implicitly instead of fully explicitly. Thus, using this justification, Eq. (3.3.3) is rewritten as

$$
\rho^{n}\left(\frac{u^{n+1}-u^{*}}{\Delta t}\right)=-\underset{\sim}{\nabla}\left(P^{n+1}-P^{n}\right)
$$

Special care must be taken to ensure that the density term in Eq. (3.3.4) never equals zero, as this would cause singularities in the solution. Thus, taking the divergence of Eq. (3.3.4) and assuming that $\mathrm{u}^{\mathrm{n}+1}$ satisfies continuity yields

$$
-\underset{\sim}{\nabla^{2}}\left(P^{n+1}-P^{n}\right)=\frac{1}{\Delta t}\left[\underset{\sim}{\nabla}\left[\left(\rho^{n} u^{*}\right)+\frac{\partial \rho^{n+1}}{\partial t}\right]\right.
$$

Thus, once $\mathrm{P}^{\prime}\left(\mathrm{P}^{\prime}=\mathrm{P}^{\mathrm{n}+1}-\mathrm{P}^{\mathrm{n}}\right)$ is calculated from Eq. (3.3.5), the velocity field and pressure field at the $n+1$ time level are calculated, respectively, from

$$
\begin{aligned}
& u^{n+1}=u^{*}-\frac{\Delta t}{\rho^{n}} \underset{\sim}{\nabla} P^{\prime} \\
& P^{n+1}=P^{n}+P^{\prime}
\end{aligned}
$$

Thus, the general formulation for the projection method is to guess an initial pressure field and solve for an initial velocity field, then project that velocity field onto a new pressure field. Use the new velocity field and correct for the pressure, then calculate the corrected velocity field. Although this is the general formulation, there are many other ways to achieve the same goal.

\subsection{Solution of System of Algebraic Equations}

DREAM $^{\circledR}$, as well as other commercially available CFD codes, produces large sets of equations from the momentum and Poisson's equation. The general form of the linear system of equations is

$$
A \vec{x}=\vec{b}
$$

where $\mathrm{A}$ is a square matrix of order $\mathrm{N}, \mathrm{x}$ is the solution vector, and $\mathrm{b}$ is the right hand side of the equations. The computational efficiency of any CFD code is highly dependent on the use of an appropriate equation solver, which is why this section will examine the linear equation solvers available in $\operatorname{DREAM}^{\circledR}$. Generally speaking, there are three types 
of techniques available, classified as direct, semi-direct, and iterative methods. Direct methods, which are not used in $\operatorname{DREAM}^{\circledR}$, yield an exact solution, in the absence of round off errors, in a finite number of operations that can be determined in advance. These methods can be applied to any nonsingular matrix, and are well adapted to matrix inversion and linear equations.

Two semi-direct methods are available in DREAM $^{\circledR}$, being the Incomplete Cholesky Conjugate Gradient Method, ICCG, and the Bi-Conjugate Gradient Stabilized Method, BiCGSTAB. The idea of ICCG is to decompose the matrix into the form $\mathrm{LDL}^{\mathrm{T}}$, although other forms are just as possible, such as the $\mathrm{LL}^{\mathrm{T}}$. This type of form, known as the Cholesky form, has the advantage of needing less storage due to the symmetry of the matrix, and fewer operations are needed to perform the decomposition. For a detailed derivation of the ICCG method, the reader is referred to Smith (1996). The Bi-Conjugate Gradient Stabilized method is another popular solver not only because it is usable for nonsymmetrical matrices, but also because it converges smoothly where other conjugate gradient methods become unstable. Usually, the BiCGSTAB outperforms CGSTAB methods in most cases. Again, the reader is referred to (Smith (1996)) for more details of such solvers.

For most simulations performed using DREAM ${ }^{\circledR}$, the SIP3D solver is used, which stands for Strongly Implicit Procedure, developed by Stone and Kwan (1969). The SIP3D method solves large systems of equations through iteration and converts the finitedifference equations into a series of matrices. A series of sparse, symmetric matrices are formed in the process. SIP3D is more computationally efficient than the other solvers mentioned, as it has an additional acceleration parameter, which is multiplied by the residual vector. The number of iterations to convergence also tends to be less than the other solvers used in DREAM ${ }^{\circledR}$. For more information on SIP3D, the reader is referred to Stone and Kwan (1969).

\subsection{Boundary Conditions}

No discussion of numerical methods would be complete unless a discussion on boundary conditions was included. Patankar (1980) presents a review of the boundary conditions for the pressure correction methods, which our method falls into. For solid 
wall boundaries, or boundaries where the flow is known, Dirichlet conditions are to be used for the velocity. Since the velocity is known at this type of boundary, no correction need be made to it and the corresponding pressure correction boundary condition is $\partial \mathrm{P}^{\prime} / \partial \mathrm{n}=0$. If the pressure is known at a boundary, then the Dirichlet condition is used for the pressure correction, which usually becomes $\mathrm{P}^{\prime}=0$. Therefore, the corresponding boundary condition for the velocity would be a Neumann conditions, $\partial \mathrm{u} / \partial \mathrm{n}=0$.

An important point, which will be addressed in Chapter 5 of the simulations for an impinging jet, is the behavior of ITM at or near solid boundaries. Traditional LES methods, at times, have difficulties in capturing the flow characteristics in complex geometries. It may be expected that for certain flows, unless the grid resolution is fine enough to capture the boundary layer at the wall, then the simulations will contain considerable amounts of error in the solution. However, little work has been done in this field of ITM, and Chapter 6, Section 6.4.2 will try to address some of the effects of wall impingement by a curved buoyant jet on the global flow field, i.e. turbulence generation or suppression. 


\subsection{VALIDATION OF DREAM ${ }^{\circledR}$ CODE}

\subsection{Introduction}

Large eddy simulations, LES, require the use of accurate numerical schemes in order to alleviate and prevent error propagation. Generally, when considering the expansion of a function using a Taylor series, the higher the order of included terms, the more accurate the solution becomes as the truncation error is reduced. Unfortunately, this is not an accurate perception of LES, as the higher the order of accuracy accompanied with coarse grid spacing may not produce reasonable results (Rai and Moin (1991)). This section examines the performance of LES using ITM methods as implemented into DREAM ${ }^{\circledR}$. Results clearly show that with appropriate grid resolution, good agreement with available experimental data is achieved.

\subsection{LES of Flow Past a Square Cylinder}

The turbulent flow around a square cylinder has not been studied as extensively as the flow around a circular cylinder. The flow topology between the two is expected to be identical, however differences in the length and velocity scales provides crucial insight into the relationship between the coherent vortex structures and the random turbulence characteristics. In order to determine the performance of ITM methods utilized in DREAM $^{\circledR}$, experiments performed by Lyn et. al. 1995), accompanied with numerical simulations by Rodi (1997), Sohankar et. al. (2000), and Shi (2001) will be used to quantify various flow field properties. The geometry used in the DREAM ${ }^{\circledR}$ simulations is shown in Figure 4.2.1. The Reynolds number, based on the side length of the square cylinder and inlet velocity, for all experiments and simulations was 22000. A uniform grid of $300 \times 200 \times 20$ in the streamwise, vertical and spanwise directions, respectively, was used. This corresponds to a uniform cell size of $0.005 \mathrm{~m} \times 0.005 \mathrm{~m} \times 0.025 \mathrm{~m}$. This type of grid was used, because as Figure 4.2.1 indicates, the square cylinder traverses the entire spanwise direction, and thus the fluctuations are assumed to be homogenous in the spanwise direction, which justifies the use of a 5:1 grid ratio in the simulations. Most 
fluctuations are in the streamwise and vertical directions, thus the increased grid density in those directions.

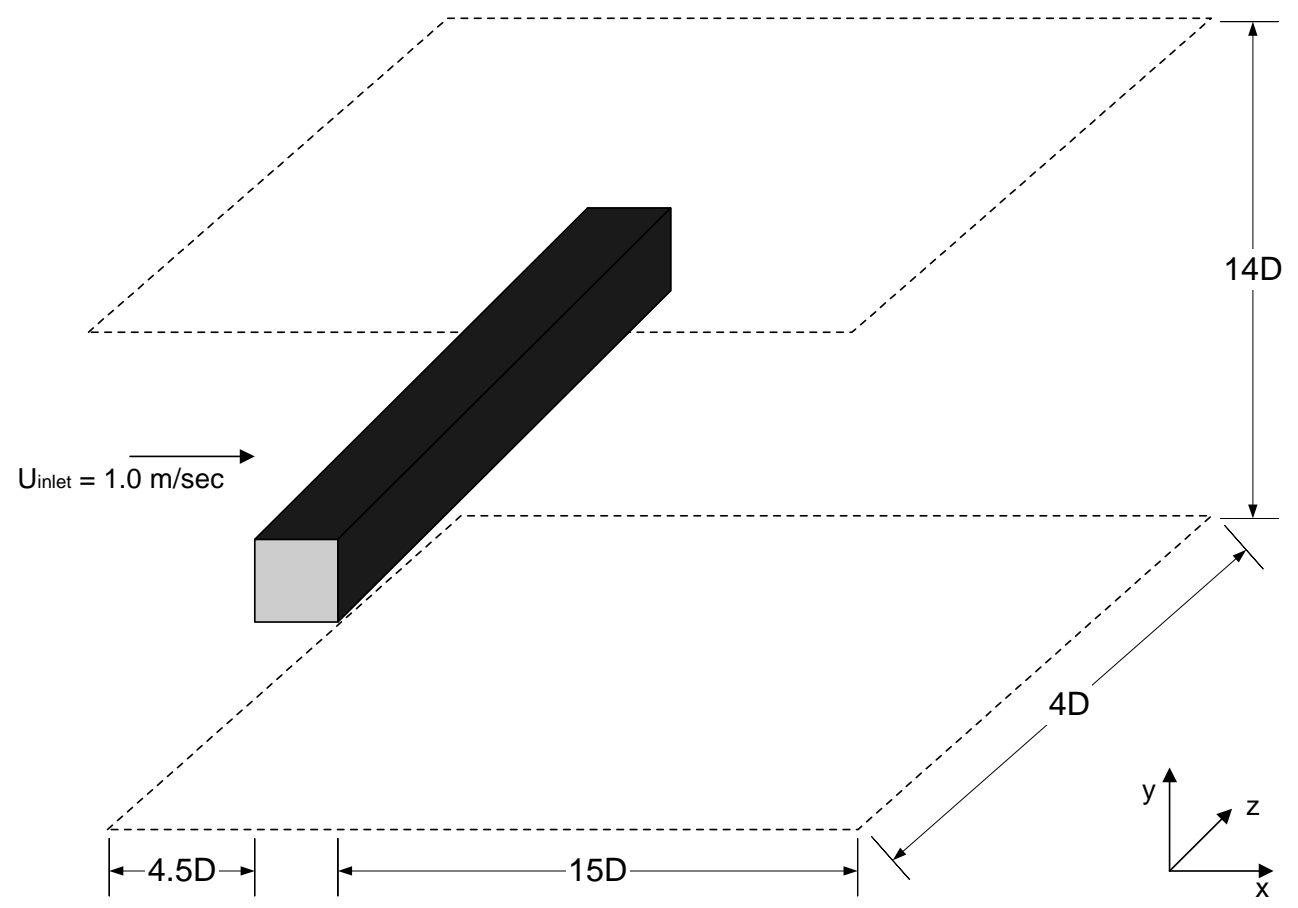

Figure 4.2.1 - The geometry of the flow past a square cylinder

In the simulations by Shi (2001) and Rodi (1997), non-uniform grids were used, which gave a higher resolution near the cylinder. A uniform grid was used for the DREAM $^{\circledR}$ simulations, which required a much larger number of grids throughout the entire domain to get equivalent grid spacing to those of the previous studies in the streamwise direction around the cylinder. However, an increase in the overall grid size allowed for a higher resolution of the far field domain downstream of the cylinder. Symmetry boundary conditions were applied in the vertical direction, with inflow and continuum boundary conditions being used in the direction normal to the streamwise plane. Periodic boundary conditions were applied in the spanwise direction. A no-slip boundary condition is applied at the surface of the square cylinder. Application of these boundary conditions assumes that the vortex shedding is equivalent in the $\mathrm{z}$ direction.

Analysis of the mean streamwise velocity distribution from the ITM simulations, see Figure 4.2.2, shows good agreement with the available experimental data. The total 
averaging time is taken to be five times the period that corresponds to the shedding frequency. The simulations by Shi (2001) are less accurate downstream of the square cylinder compared to the present results, which probably is due to the increased resolution used in the DREAM ${ }^{\circledR}$ simulations. The high degree of fluctuations observed in the mean velocity calculations by Shi (2001) is most likely due to the lack of using sufficiently long averaging times and is clearly a statistical phenomenon. Unfortunately, it would be difficult to use an equivalent as from the Shi (2001) simulations because the resolution of the square cylinder would be reduced.

Although the improvement in using the ITM methods in DREAM ${ }^{\circledR}$ is less noticeable in Figure 4.2.2, the improvements become evident in Figure 4.2.3, where the streamwise turbulence intensity for the Shi (2001) and Lyn et. al. (1994) data are shown. Both simulations perform well in the location just downstream of the cylinder, but the DREAM $^{\circledR}$ results are slightly better than those by Shi (2001) in the far field, which is again most likely due to the increased grid resolution. Figure 4.2.4 shows a similar trend for the vertical velocity component of the turbulence intensity. Figure 4.2.5 shows the $\mathrm{z}$ component of the vorticity and Figure 4.2.6 shows the streamlines at the center plane to which the cylinder is normal. These figures exhibit very similar features that are also obtained by others, see. e.g. Rodi (1997) and Shi (2001)

The results from the present simulations, when compared to the available experimental and computational data, lend weight to the initial hypothesis concerning ITM used in this study. Thus, when an appropriate grid resolution is used, in conjunction with second-order, monotonic, control volume discretization technique, ITM is capable of simulating turbulent flows. However, reduction in the time step used in the simulations will further decrease the amount of dissipation present within the simulations. However, it is clearly shown that the large scale vertical structures are well captured. This statement is further supported in the next section. Although this will limit the efficiency of the solver, it becomes necessary because of the utilization of the projection method in the handling of the velocity-pressure coupling. 


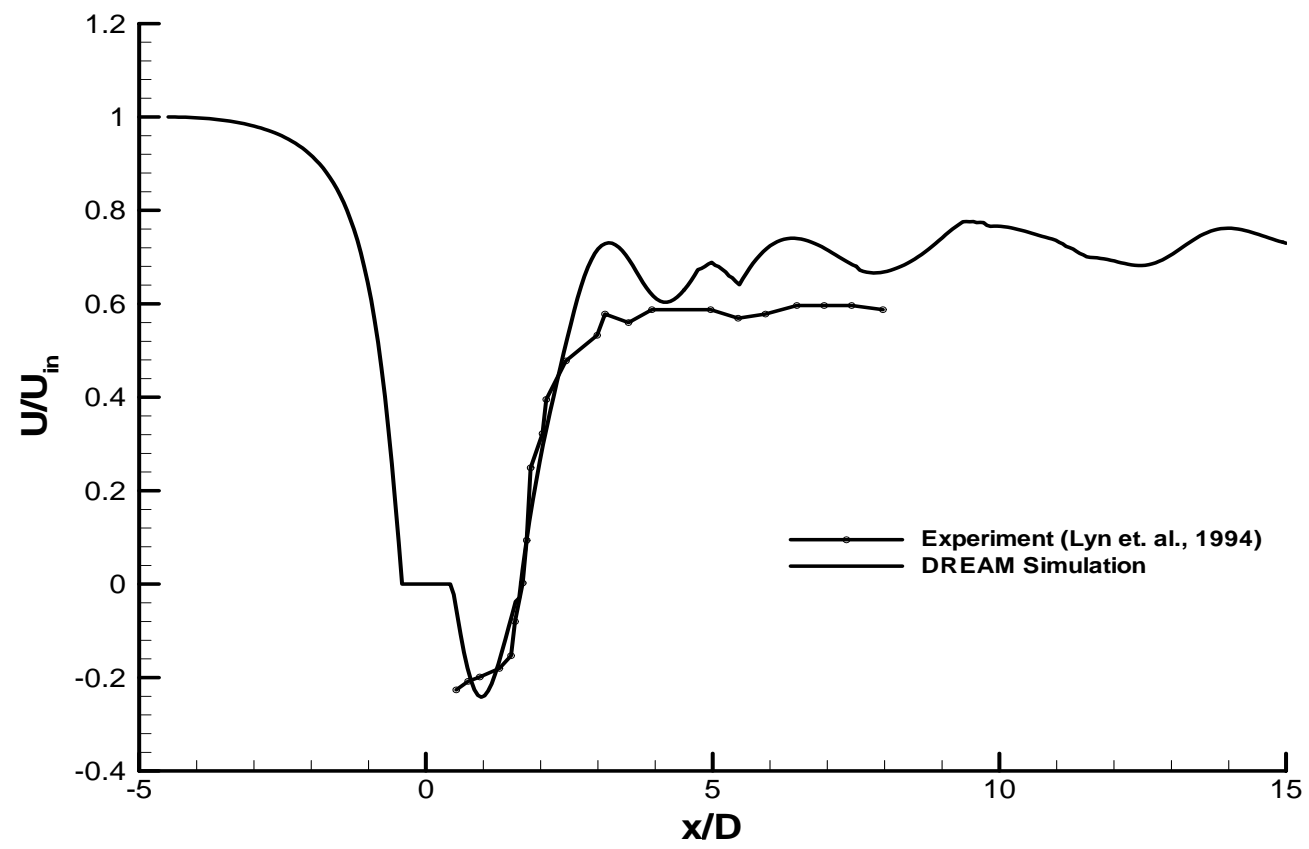

(a)

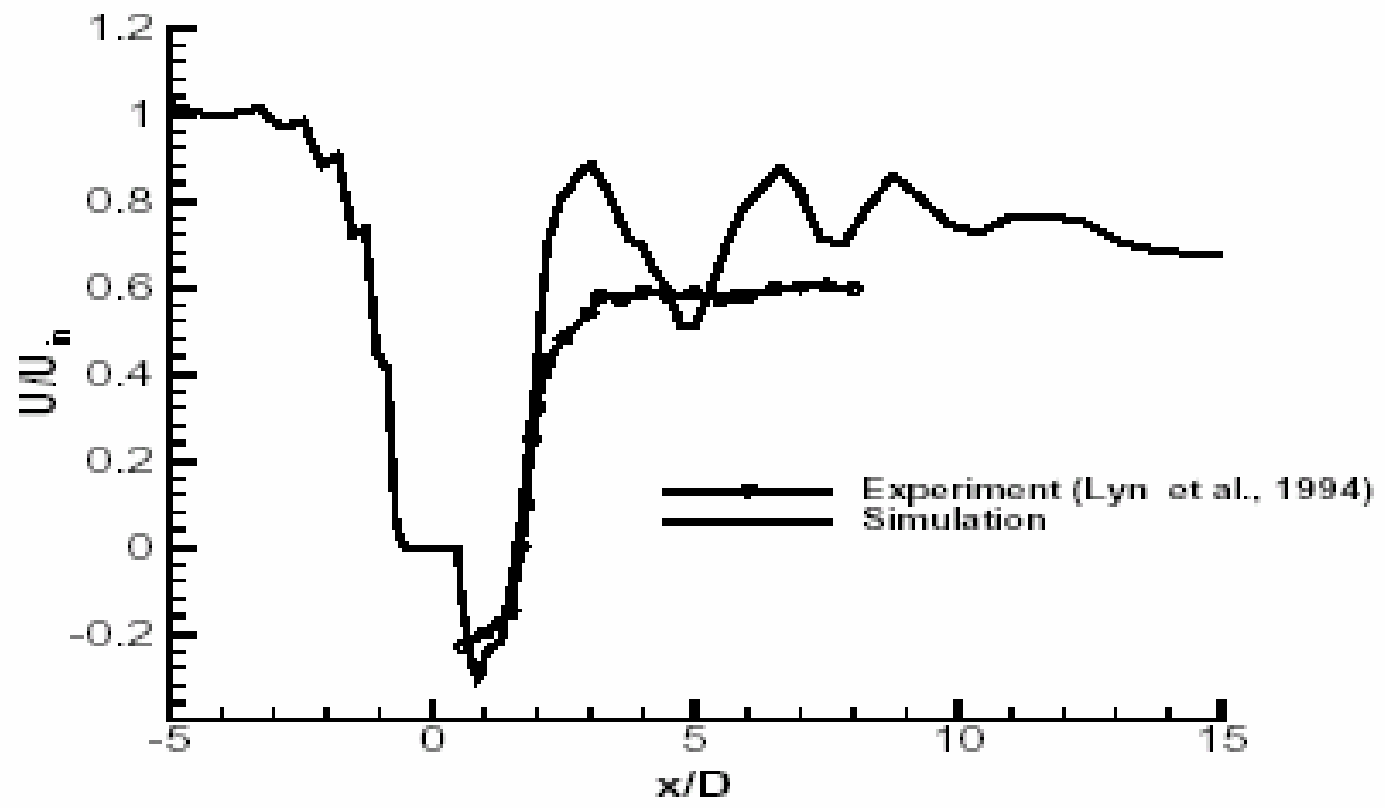

(b)

Figure 4.2.2 - Mean streamwise velocity distribution on the center plane of the cylinder. The experiments are by Lyn et al. (1994) and the data starts behind the cylinder. The cylinder is located between $-1<\mathrm{x} / \mathrm{D}<1$. (a) DREAM ${ }^{\circledR}$ simulations (b) The corresponding simulations are from Shi (2001) using the QUICK scheme with the Smagorinsky SGS model. 


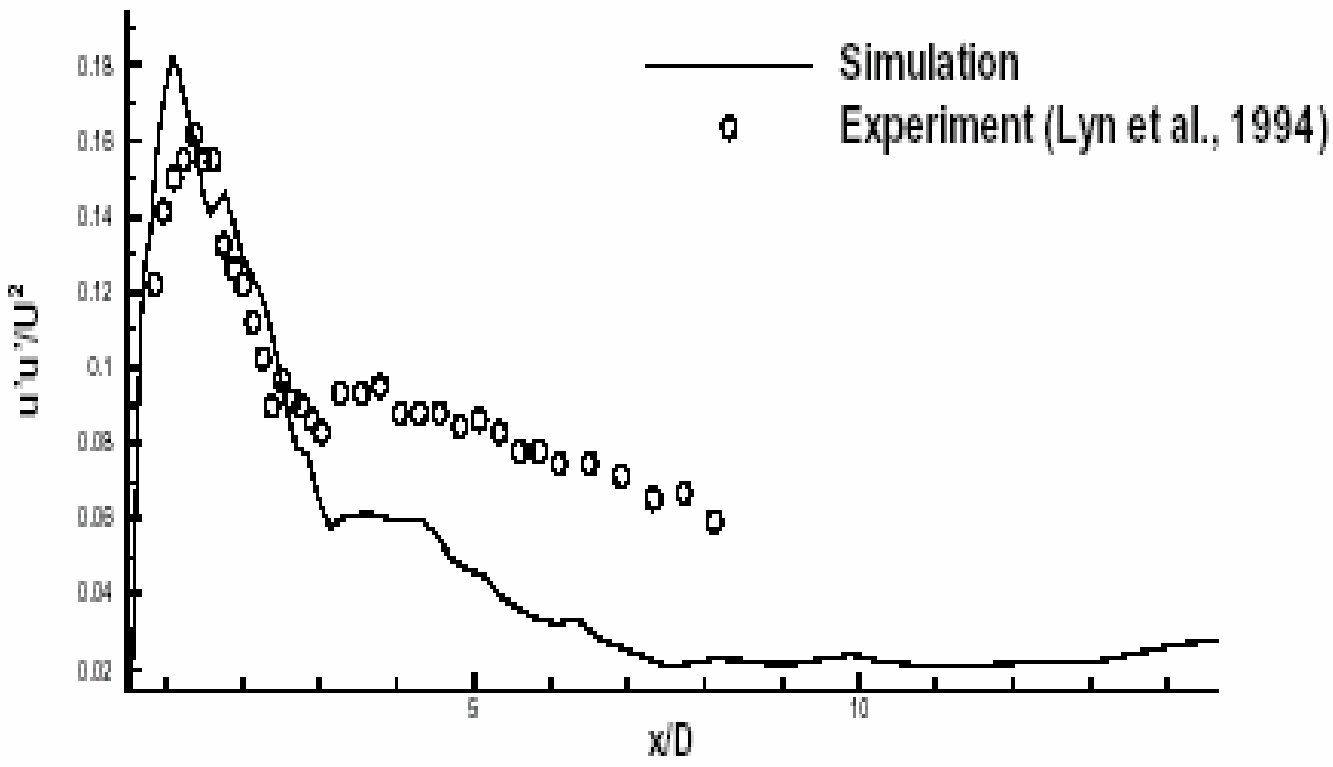

(a)

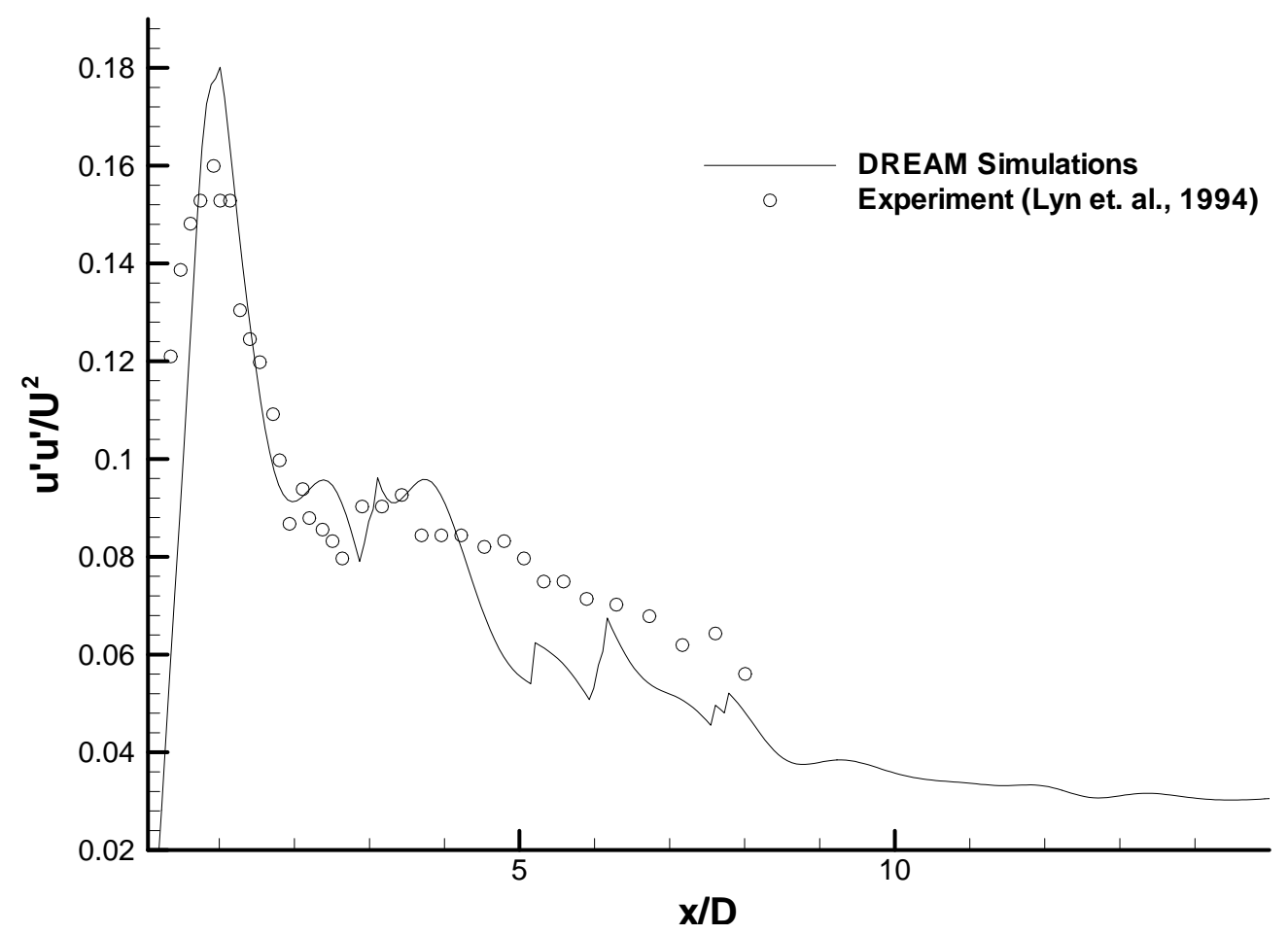

(b)

Figure 4.2.3 - Streamwise velocity component of the turbulence intensity of the flow past a square cylinder. (a) The corresponding simulations are from Shi (2001) using the QUICK scheme with Smagorinsky SGS model. (b) DREAM ${ }^{\circledR}$ simulations 


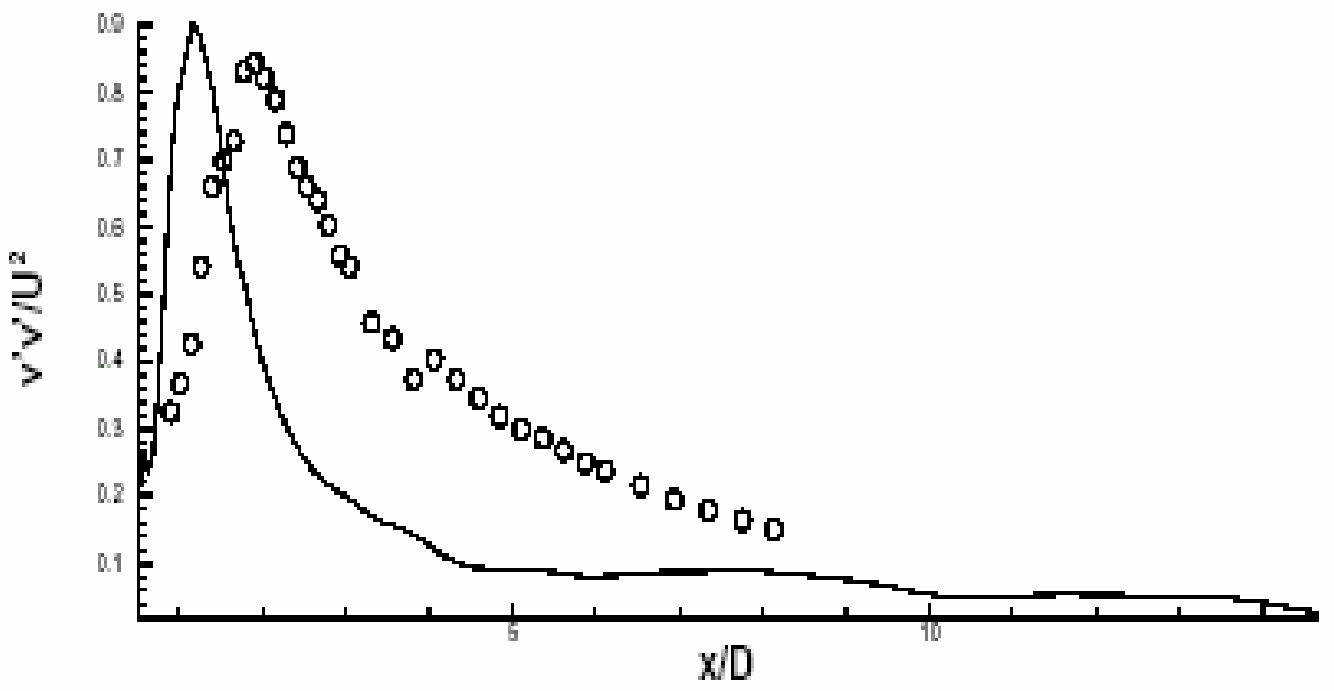

(a)

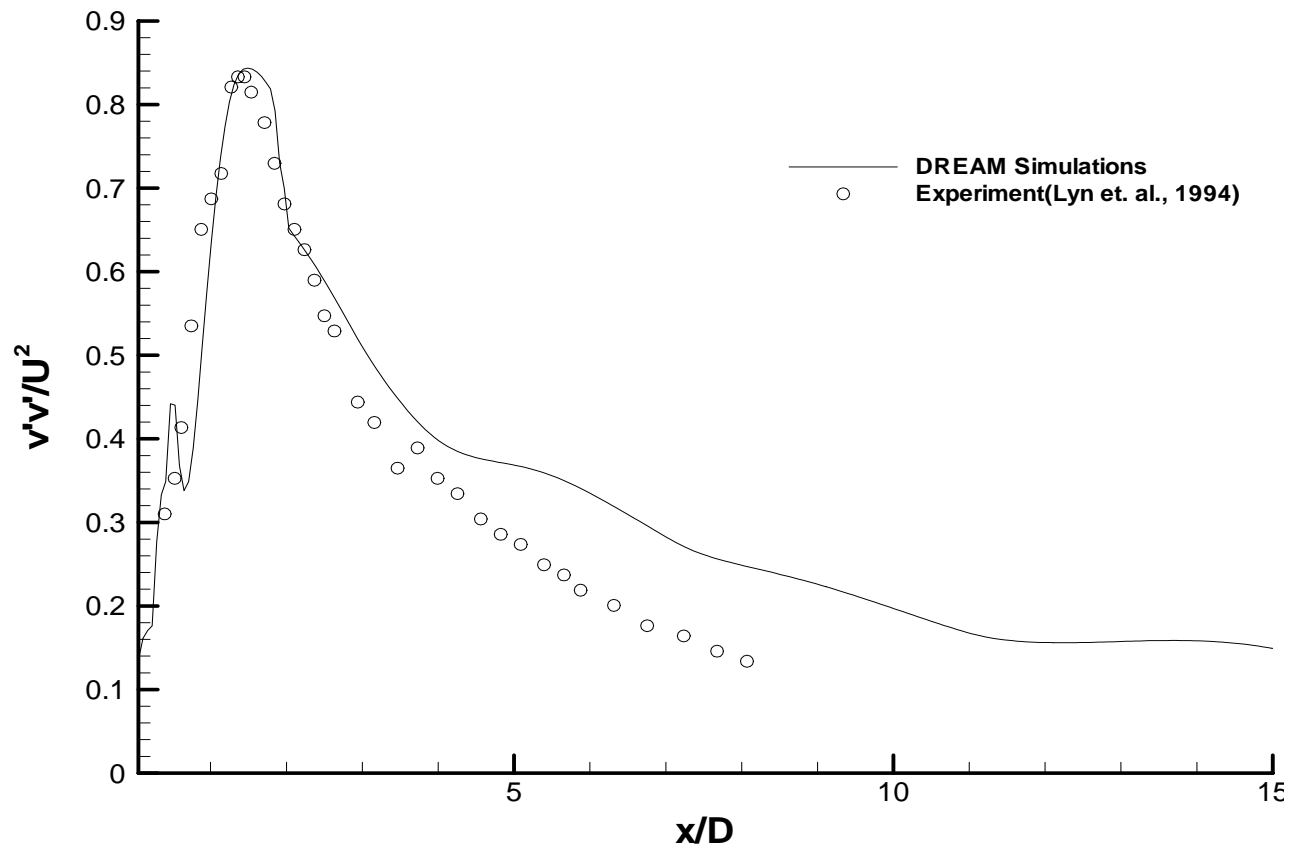

(b)

Figure 4.2.4 - Vertical velocity component of the turbulence intensity of the flow past a square cylinder. (a) The corresponding simulations are from Shi (2001) using the QUICK scheme with Smagorinsky SGS model. (b) DREAM ${ }^{\circledR}$ simulations 


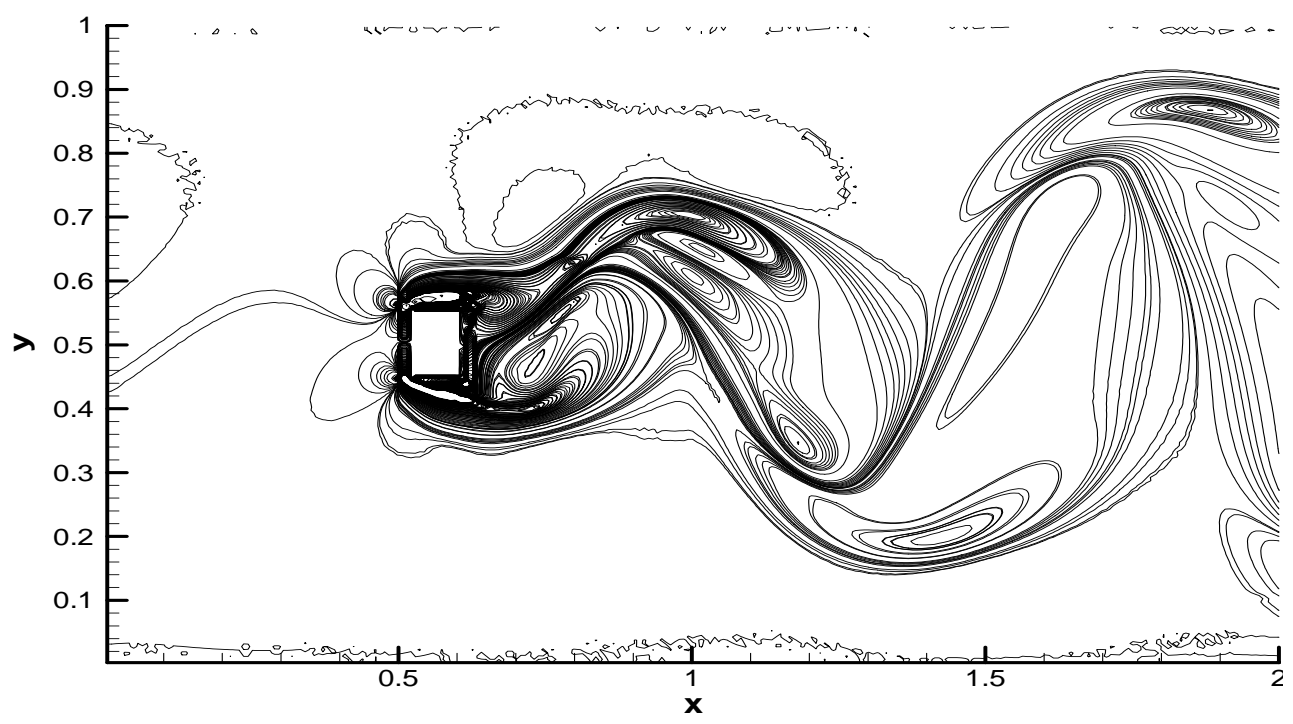

Figure 4.2.5 - $\mathrm{z}$ component vorticity of the flow past a square cylinder for the DREAM ${ }^{\circledR}$ simulations

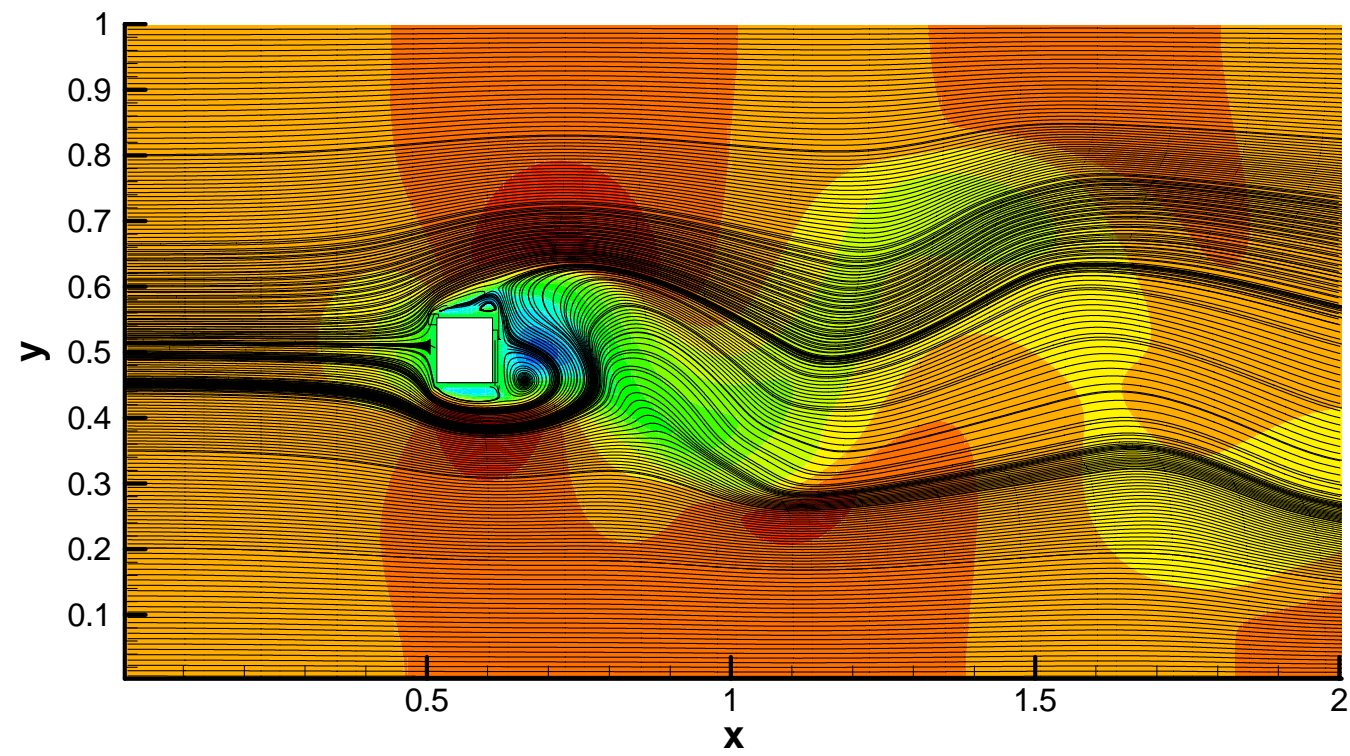

Figure 4.2.6 - Center plane streamlines for the flow past a square cylinder using DREAM $^{\circledR}$ 


\subsection{LES of Plane Mixing Layer}

The study of mixing layer flows created from a variable velocity inlet condition allows for a more in-depth analysis of the capabilities of DREAM ${ }^{\circledR}$. The experiments from Rightley (1995) will be used in the quantification of the simulations using ITM, along with those from Shi (2001). Original studies were performed to investigate the behavior of bubble dynamics in a turbulent shear layers; however, only the resulting turbulent flow field is examined in this study. A non-uniform grid size of $194 \times 66 \times 42$ in Shi's (2001) simulations. Thus, it is important to note that a direct comparison to the Shi simulations is not possible; however, comparison does lend weight to the ability of DREAM to solve for these types of flows.

The measurement domain used in the experiments by Rightley (1995) is shown in Figure 4.3.1, where dimensions are $0.55 \mathrm{~m} \times 0.2 \mathrm{~m} \times 0.2 \mathrm{~m}$ in the $\mathrm{x}, \mathrm{y}$, and $\mathrm{z}$ directions, respectively. These dimensions are also used in Shi's (2001) simulations and the present work.

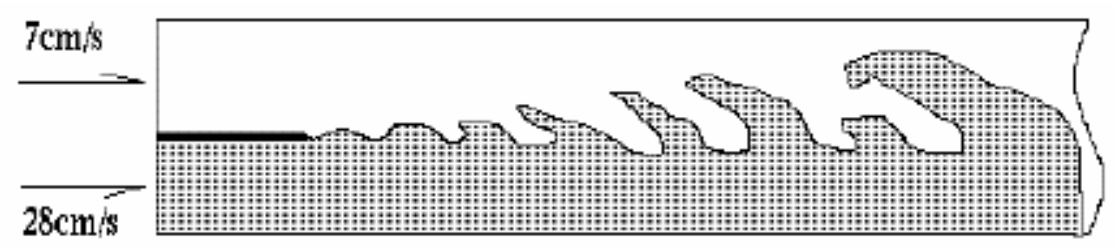

Figure 4.3.1 - Schematic of plane mixing layer domain (Shi (2001))

The mixing layer is generated due to the interfacial shear resulting from the inlet velocity difference. A splitter plate is located in the middle of the vertical plane, with the length and width of the plate equals $0.15 \mathrm{~m}$ and $0.003 \mathrm{~m}$, respectively, spanning the entire transverse direction. An inlet velocity of $0.28 \mathrm{~m} / \mathrm{s}$ is specified for the lower layer and $0.07 \mathrm{~m} / \mathrm{s}$ for the upper one, with a standard outflow boundary condition applied at the outlet of the domain. A sinusoidal driving force of five percent of the mean flow is added to the vertical velocity component of the lower layer. The amplitude of the forcing frequency is 0.014 , with the driving frequency being $2.2 \mathrm{~Hz}$. This driving frequency is chosen to ensure that at least two Kelvin-Helmholtz waves are present within the test area. Also, all of the turbulence arises from the shear between the two velocity layers, 
and the driving frequency is utilized to ensure than more than one Kelvin-Helmholtz wave is present within the test section.

For the DREAM ${ }^{\circledR}$ simulations, two different uniform grids were used in the simulations, which were $200 \times 80 \times 40$ and $300 \times 120 \times 60$ in the $\mathrm{x}, \mathrm{y}$, and $\mathrm{z}$ directions, respectively. Slip-wall boundary conditions are used in the vertical and spanwise directions. In the $\mathrm{DREAM}^{\circledR}$ simulations, the flat plate is represented by a region of zero velocity. This treatment corresponds effectively to a no-slip boundary condition. In the simulations by Shi (2001), slip wall boundary conditions are used; however, it is the opinion of this author that using a no-slip boundary in the simulations is more appropriate. Treating the splitter plate as a flat plate helps in the global flow field determination, as well as the fact that the end of the splitter plate induces fluctuations, which may only be captured using a zero-velocity boundary condition. Shi (2001) uses a Smagorinsky sub-grid scale model, where as the DREAM ${ }^{\circledR}$ simulations use ITM type formulation, with no sub-grid scale model.

Although the experiments were intended to investigate the behavior of bubbles within a mixing layer, the bubble concentration was kept low enough so as to eliminate dual coupling. This makes it possible to treat the bubbles as marker particles, having no effect on the flow field and will not alter the mean and root mean square velocity distributions. Data analysis from the experiments takes place at three different $\mathrm{x} / \lambda$ locations being $1.88,2.5$, and 3.66 , where $\lambda$ is a characteristic length scale equal to the shear layer width. These test regions correspond to $15,20,25 \mathrm{~cm}$ downstream of the splitter plate end.

Instantaneous velocity vectors are shown in figures 4.3.2 - 4.3.4 for three different vertical locations from Rightley (1995), Shi (2001) and the current DREAM ${ }^{\circledR}$ simulations. These vectors are for the differential velocity field obtained by subtracting the mean velocity. Comparison indicates a very good agreement between the three. Figures 4.3.5 - 4.3.6 depict a comparison of instantaneous contour plots from Shi (2001) and DREAM ${ }^{\circledR}$. Again, a very good agreement between the two is observed.

One of the benchmarks of LES simulations should be in the prediction of the turbulent kinetic energy. The instantaneous velocities within the shear flow are comprised of three components, being the mean flow, the average velocity of the 
coherent vortices, in this case the Kelvin-Helmholtz vortices, and the random eddy velocity fluctuations. Figure 4.3.7 shows the instantaneous velocity at two different points within the flow field. Clearly, the fluctuations in the velocities indicate the presence of turbulence within the flow, with the bulk flow being from the vortical structures present within the shear layer. Experiments found that the frequency of the Kelvin-Helmholtz waves equaled $2.2 \mathrm{~Hz}$, with analysis of the results from DREAM ${ }^{\circledR}$ this was found to be $2.41 \mathrm{~Hz}$, which is clearly visible in the spectral analysis (see Figures 4.3.15 - 4.3.16). Therefore, the averaging time of the data to determine the appropriate velocity component root mean square and average values was taken at least 5 times the vortex shedding period. The mean flow is also indicated on the figure. The spanwise and vertical rms and mean velocity calculations within the shear layer are depicted in Figures 4.3.8 - 4.3.13, along with the experimental data. Two different results from two different grids are presented from the current simulations, and clearly an improvement is achieved when grid refinement is performed. In the coarse grid simulations, depicted in Figure 4.3.8 and 4.3.12, the DREAM simulations appear to be shifted, with the peaks being at the centerline of the test section, although the magnitude of the peak is more accurately resolved than the Shi (2001) simulations. This is due to the inability of the uniform grid to accurately capture the effects at the centerline as well as the non-uniform grid used by Shi (2001). However, a clear improvement in the data is seen for the fine grid simulations, as the grid resolution at the interface is enhanced. The DREAM ${ }^{\circledR}$ simulations appear to perform equal or better than the simulations by Shi (2001) over most of the domain. The reason lies in the fact that a uniform grid distribution was used in the DREAM ${ }^{\circledR}$ code; as more grids are needed over the entire domain to equal those used within the shear layer by Shi (2001). It is also possible that the current treatment of the splitter plate improve the overall quality of the DREAM ${ }^{\circledR}$ results.

Unfortunately, the simulations by Shi (2001), and the experiments by Rightley (1995), do not explicitly present turbulent kinetic energy or the rms spanwise velocity component. Therefore, an approximation to the experimental data was performed to allow for determination of the kinetic energy. Vreman et. al. (1997) showed that the spanwise rms velocity fluctuations are approximately equal to the vertical rms velocity fluctuations for shear layer flows. Therefore, for quantification purposes, the vertical rms 
velocity fluctuations will be used, allowing for an approximation of the turbulent kinetic energy from the available experimental data in Rightley (1995). Although this will in fact introduce some error into the analysis, it is believed to be small. The turbulent kinetic energy comparison between the DREAM ${ }^{\circledR}$ simulations $(300 \times 120$ x 60 and $200 \times 80$ x 40) and the approximated experimental data is shown in Figure 4.3.14.

One of the important qualifications in using a ITM type LES methodology is in the determination of the energy spectrum, which provides information into the simulations not attainable through any other means. It is known that turbulence usually receives its energy at the large scales, while dissipation of the turbulent kinetic energy takes place at the small scales. The range of eddy sizes that are not affected by the energy maintenance and dissipation mechanisms is called the inertial subrange (Tennekes and Lumley, 1999). For the fine grid solution, the resolvable length scale based on the grid size equals $0.1833 \mathrm{~cm} \times 0.1667 \mathrm{~cm} \times 0.33 \mathrm{~cm}$ and $0.275 \mathrm{~cm} \times 0.275 \mathrm{~cm} \times 0.5 \mathrm{~cm}$ for the coarser grid solution. Thus, after computing the dissipation of turbulent kinetic energy, which was approximated by $u^{3} / l$, and $l$ is the integral scale assumed to be 0.1 times the thickness of the shear layer and $\mathrm{u}$ is the r.m.s velocity. Approximating the viscous length scale gives a range of 20 to 40 times less than the resolvable length scales used in the DREAM ${ }^{\circledR}$ simulations for the finest grid, and 30 to 50 times less for the coarser grid. This falls into the accepted range for ITM simulations (Oran and Boris, 1993). The details and mathematics used in the analysis of the turbulence quantities may be found in Appendix A.6. They are not explicitly presented in this section because similar treatment is utilized for the other test cases presented in later sections.

In order to use ITM in LES, the energy spectrum must predict the inertial range. The streamwise and vertical fluctuation power spectra from Rightley (1995) are shown in Figure 4.3.15, with the results from the $300 \times 120 \times 60$ DREAM $^{\circledR}$ simulations being shown in Figure 4.3.16. Analysis of Figure 4.3.16a clearly shows the prediction of the energy spectrum within the inertial subrange, which validates the simulations being presented, as well as the methodology behind the calculations. Also, it is interesting to note that in viewing Figures 4.3.16 a and b, that the frequency of the Kelvin-Helmholtz waves is clearly visible at $\mathrm{f} \approx 2.4 \mathrm{~Hz}$, which is due to the perturbation of the high-speed stream at the inlet. This increases the coherence of the large scale Kelvin-Helmholtz 
structures within the spectrum. The second peak corresponds to the forcing frequency of the lower layer, which again lends weight to the simulations.

Thus, from these simulations, accompanied with a comparison to the available simulation and experimental data, it can be concluded that $\operatorname{DREAM}^{\circledR}$, using appropriate grid resolution suitable for LES simulations, is capable of predicting turbulence within the flow to appropriate length scale, without explicitly using any sub-grid scale model for the turbulence. Although most of the energy for this type of flow is due to the development of the Kelvin-Helmholtz vortical structures, fluctuations within the flow field are evident and predictable using DREAM ${ }^{\circledR}$. 
(a)

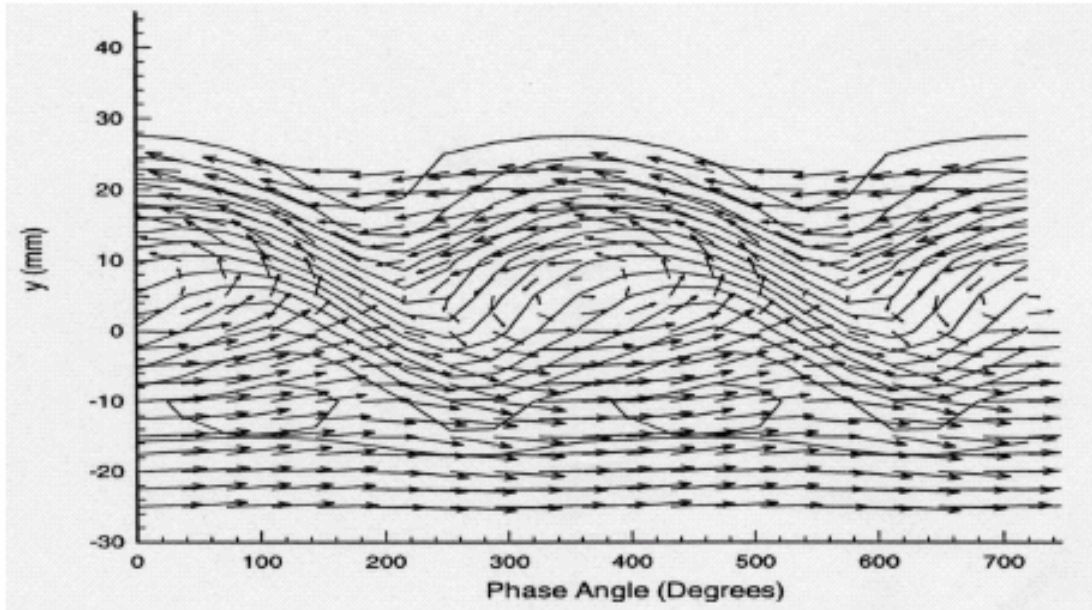

(b)

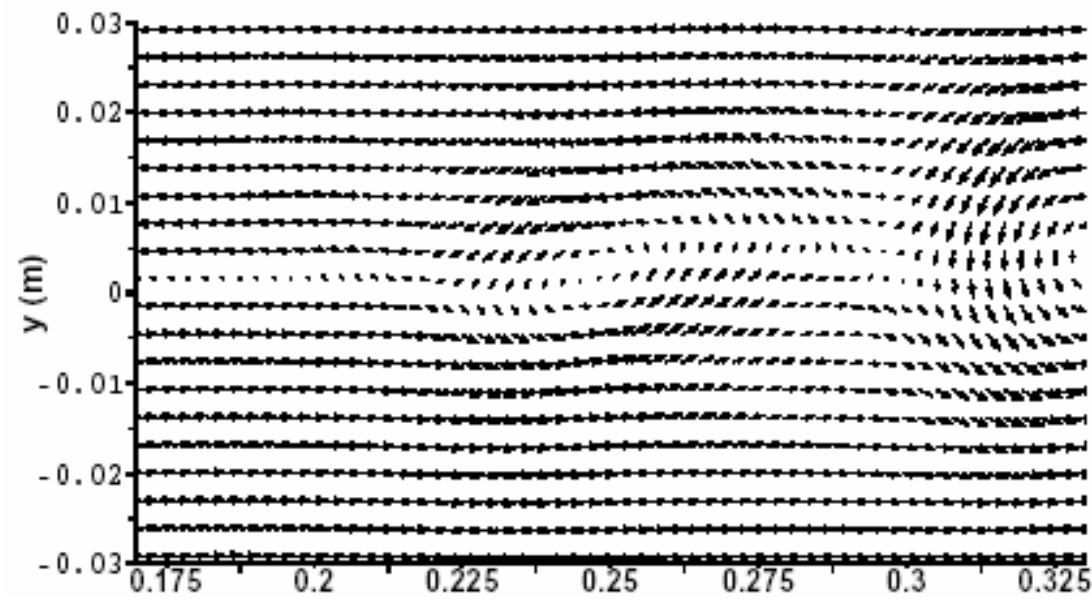

(c)

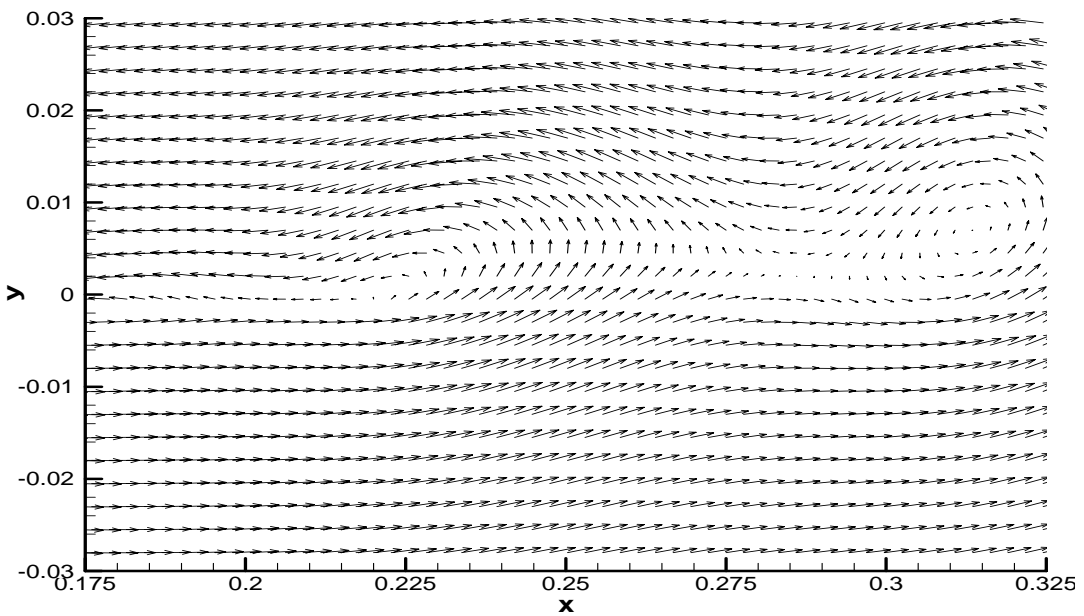

Figure 4.3.2 - Velocity vectors at $x / \lambda=1.25$ (a) Measurements by Rightley (1995); (b) Simulations by Shi (2001); (c) Present DREAM ${ }^{\circledR}$ simulations (300 x 120 x 60) 
(a)

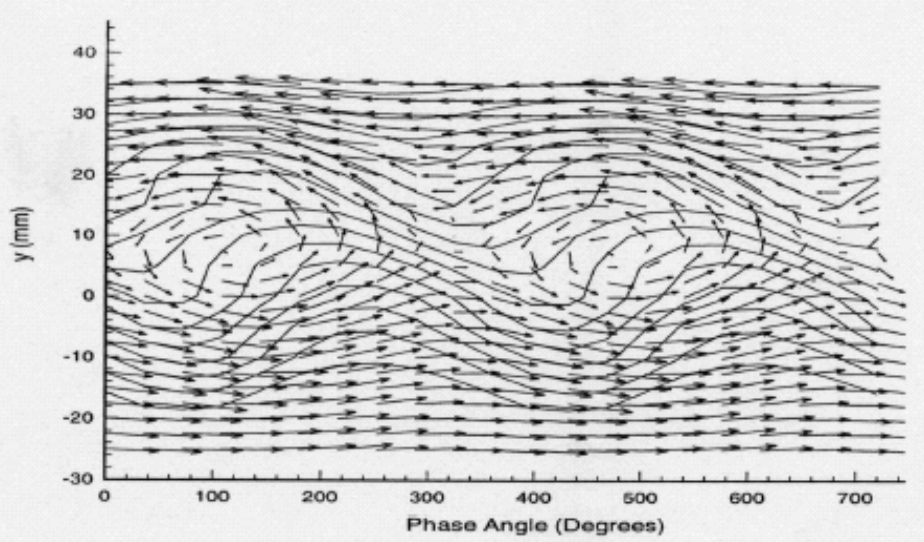

(b)
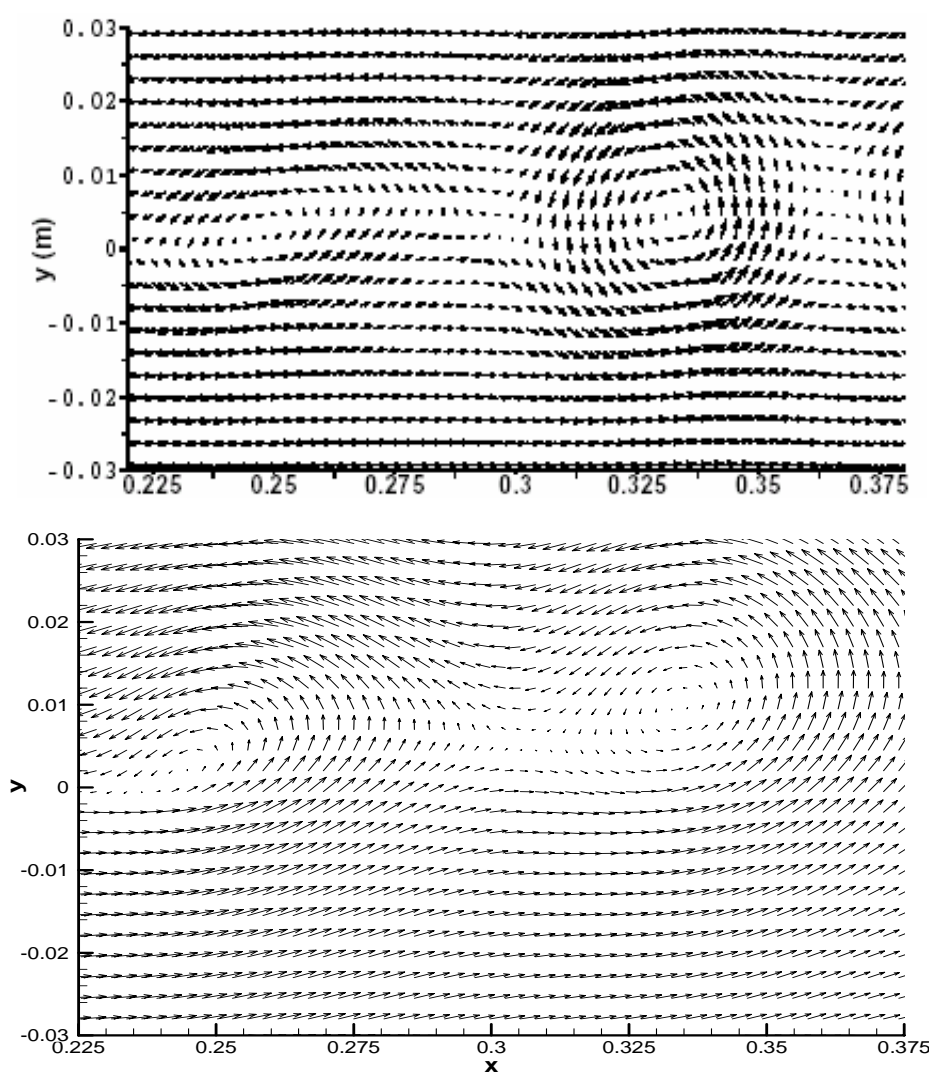

Figure 4.3.3 - Velocity vectors at $x / \lambda=1.88$ (a) Measurements by Rightley (1995); (b) Simulations by Shi (2001); (c) Present DREAM ${ }^{\circledR}$ simulations (300 x 120 x 60). 
(a)

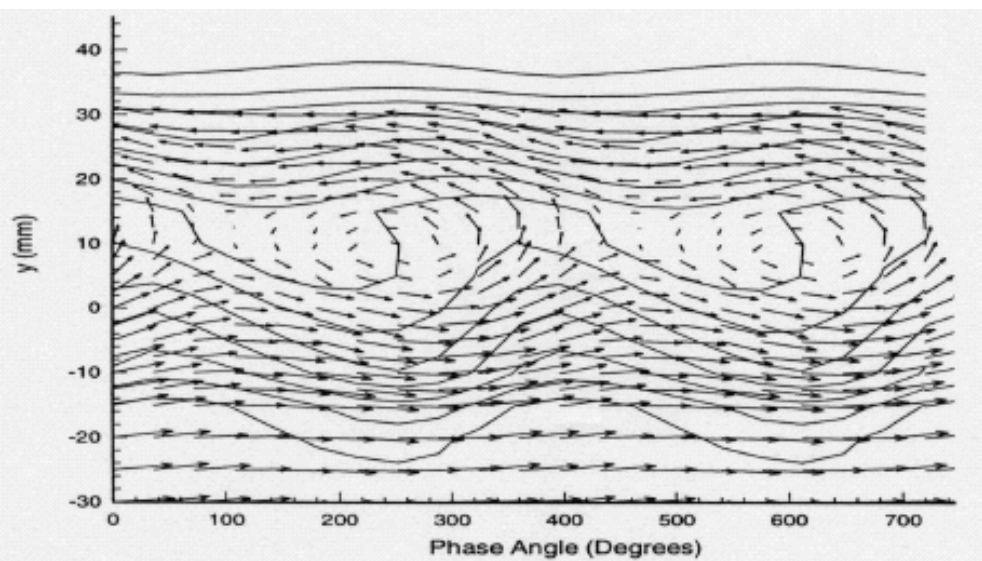

(b)

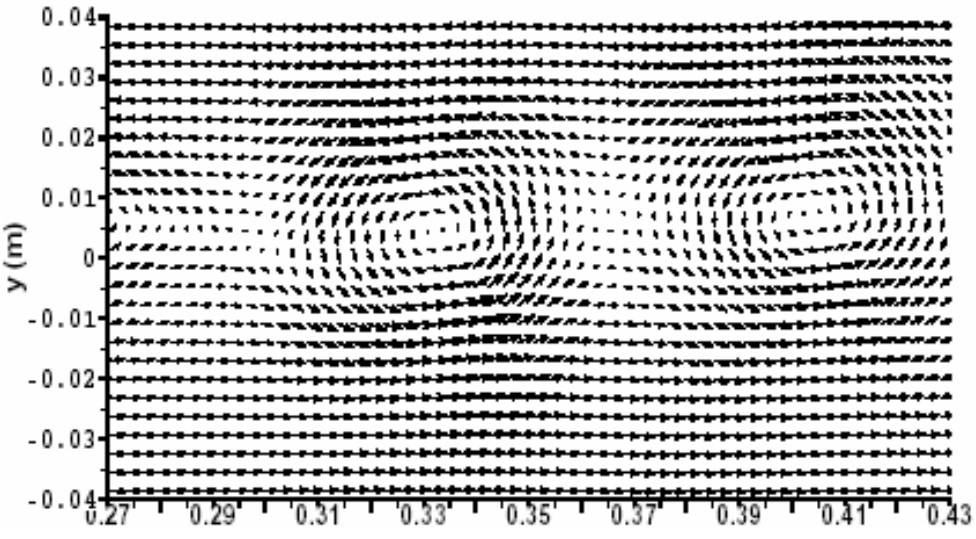

(c)

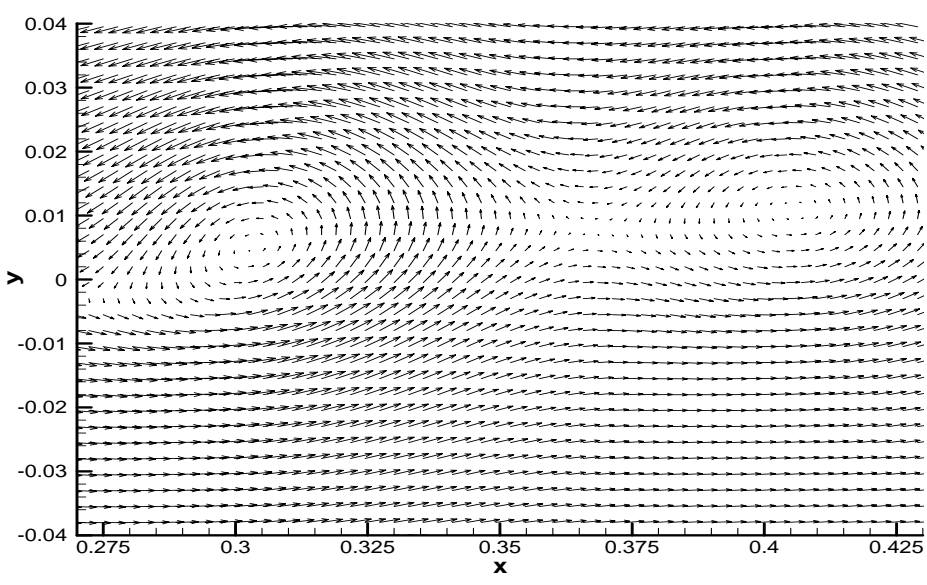

Figure 4.3.4 - Velocity vectors at $x / \lambda=2.5$ (a) Measurements by Rightley (1995); (b) Simulations by Shi (2001); (c) Present DREAM ${ }^{\circledR}$ simulations (300 x 120 x 60). 


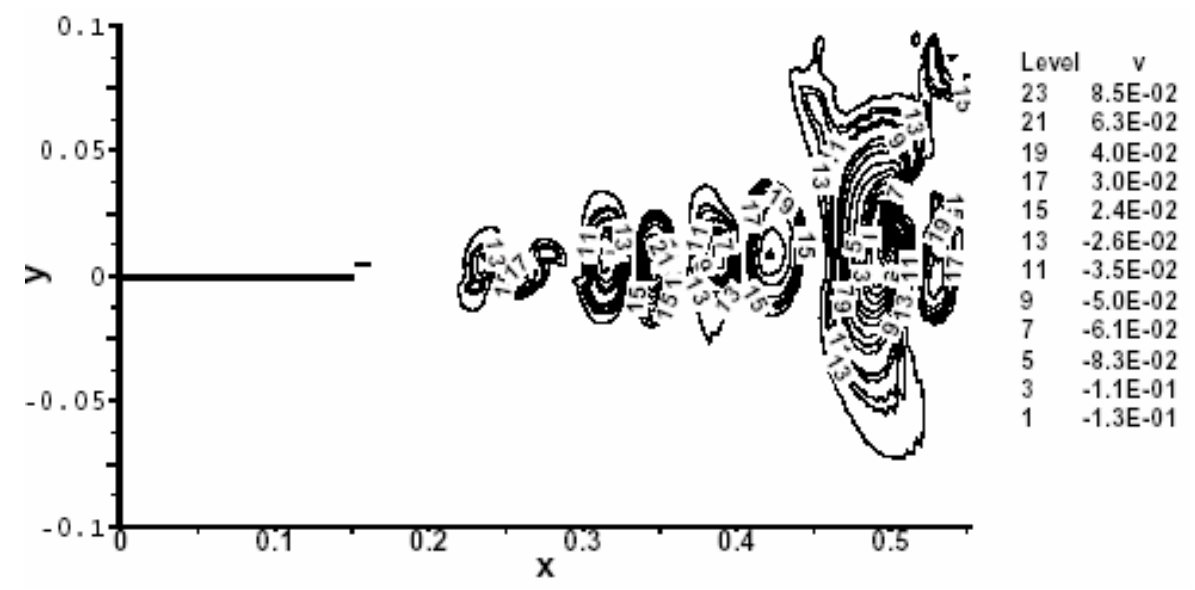

Figure 4.3.5 - Vertical velocity contours from Shi (2001)

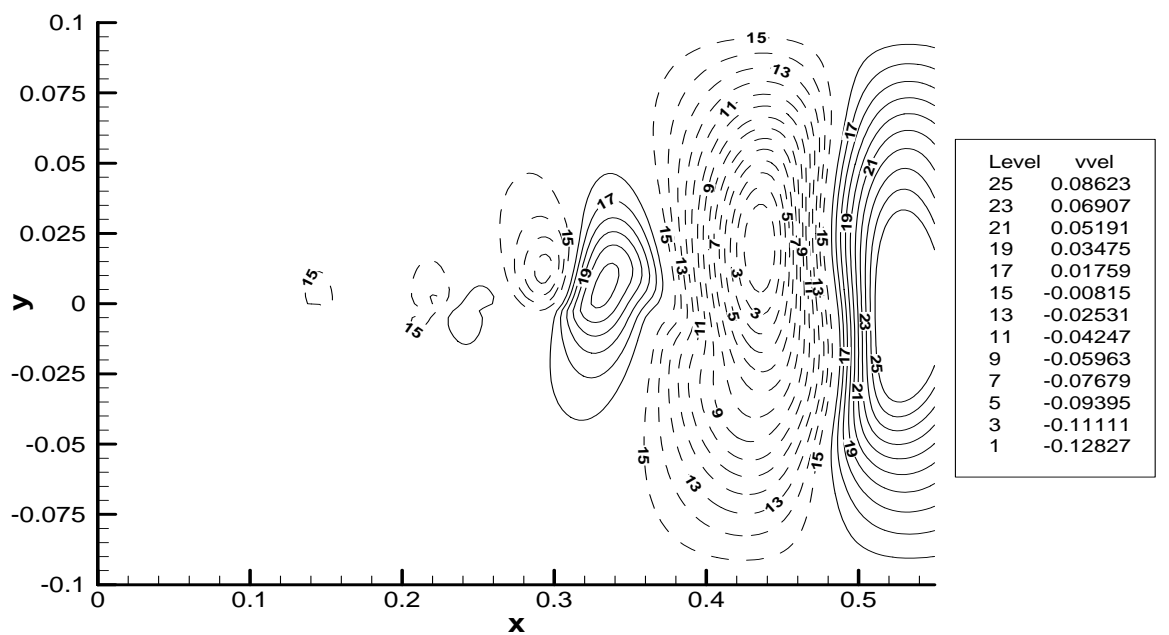

Figure 4.3.6 - Vertical velocity contours from DREAM ${ }^{\circledR}(300 \times 120 \times 60)$ 


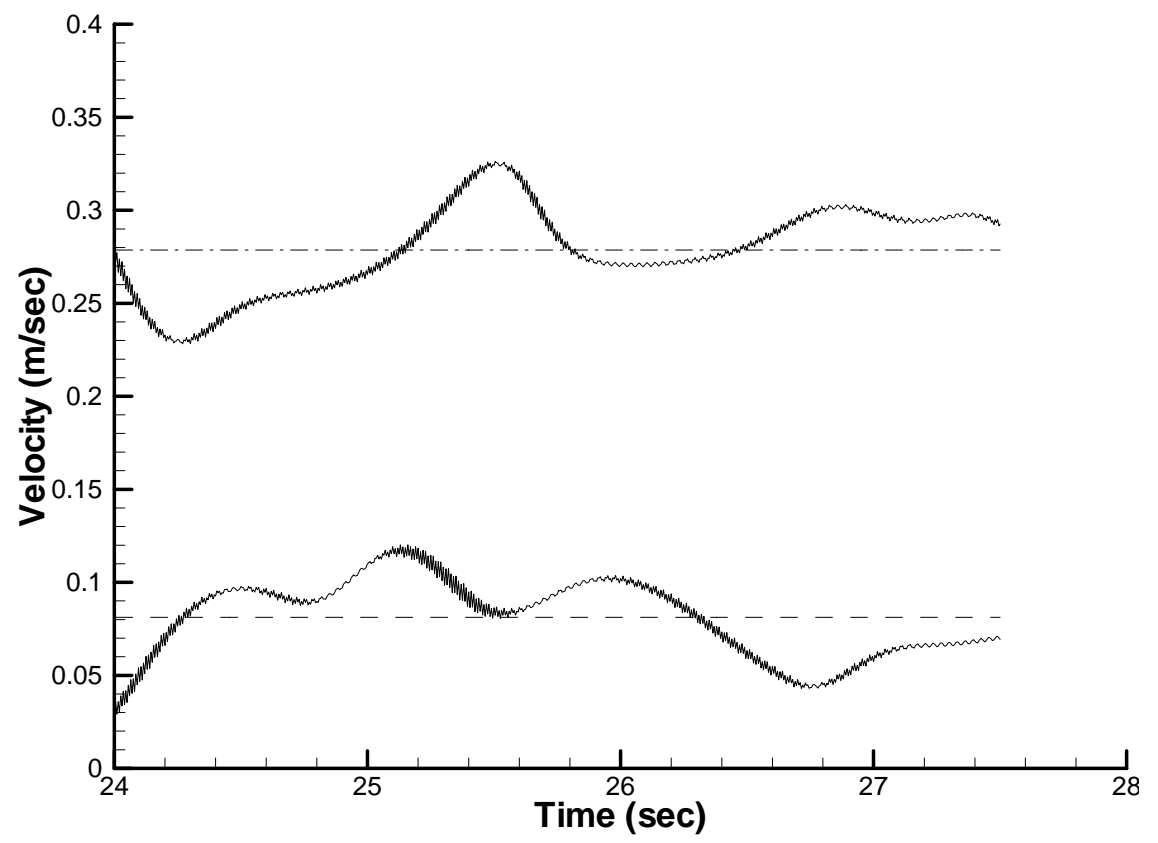

(a)

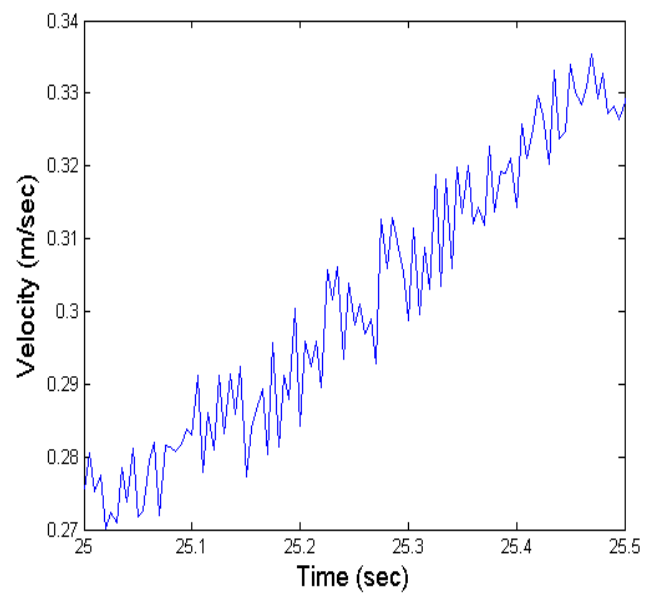

(b)

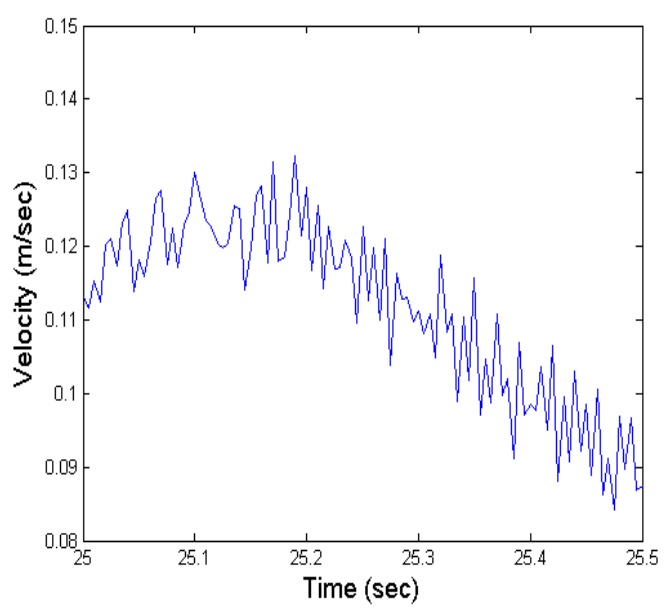

(c)

Figure 4.3.7 - (a) Mean and instantaneous velocities at $x / \lambda=1.25$ from DREAM ${ }^{\circledR}$ simulations within the mixing region. Dashed lines are mean velocities at $y=0.185$ : -----; and $y=0.210$; Solid lines are at the same locations, and represent the instantaneous velocities; (b) Zoomed in view of the $y=0.210$ profile showing turbulence fluctuations in velocity; (c) Zoomed in view of the $y=0.185$ profile. 


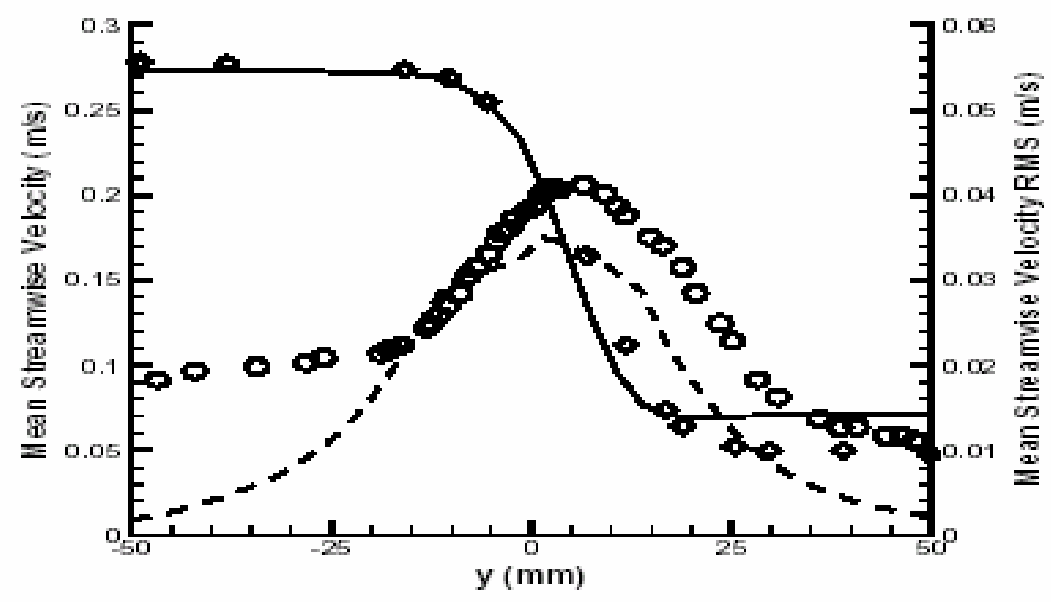

Figure 4.3.8 - Mean and $r m s$ streamwise velocity at $x / \lambda=1.25$. Symbols are from Rightley (1995): $\diamond$ : mean streamwise velocity; 0 : $\mathrm{rms}$ of streamwise velocity fluctuations. Lines from Shi (2001): : mean streamwise velocity; -----: rms of streamwise velocity fluctuations.

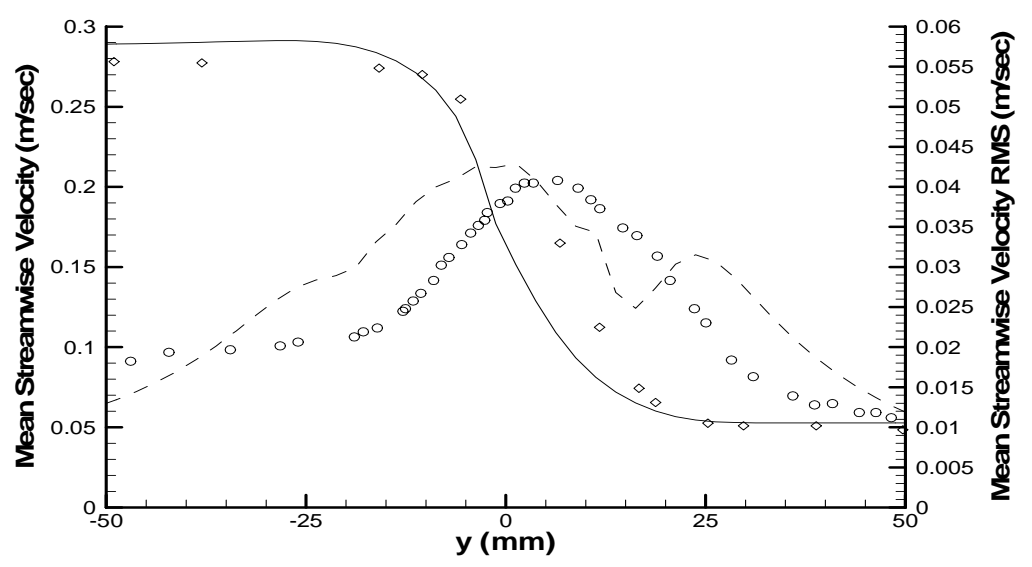

Figure 4.3.9 - Mean and $r m s$ streamwise velocity at $x / \lambda=1.25$. Symbols are from Rightley (1995): $\diamond$ : mean streamwise velocity; 0 : $r m s$ of streamwise velocity fluctuations. Lines : : mean streamwise velocity from DREAM ${ }^{\circledR}$ simulations (200x80x40); -----: $\overline{\text { rms of streamwise velocity fluctuations from DREAM }}{ }^{\circledR}$ simulations $(200 \times 80 \times 40)$. 


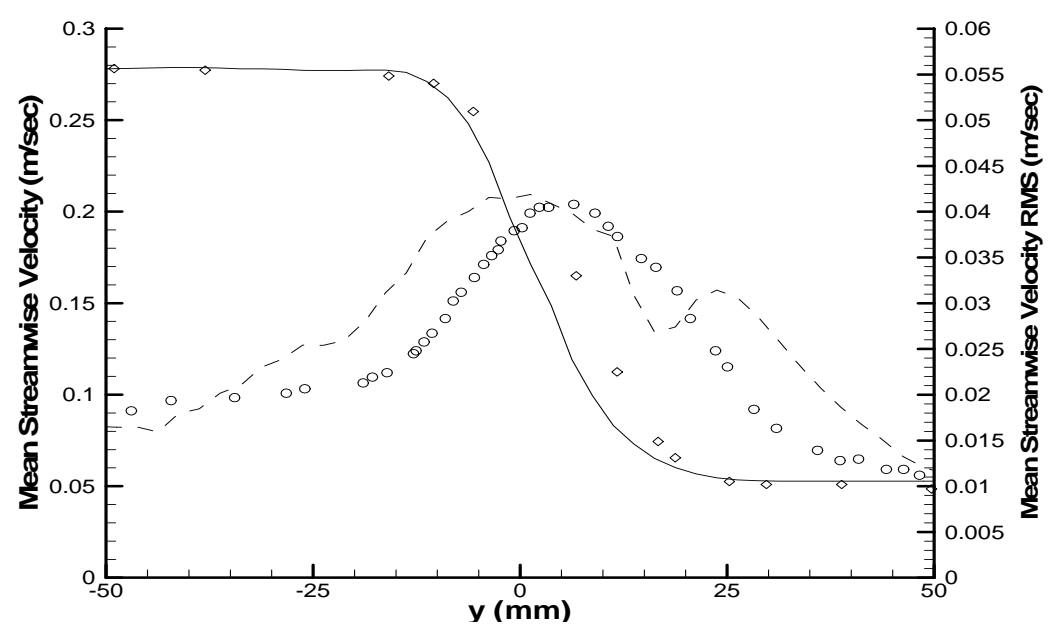

Figure 4.3.10 - Mean and $r m s$ streamwise velocity at $x / \lambda=1.25$. Symbols are from Rightley (1995): $\diamond$ : mean streamwise velocity; 0 : $r m s$ of streamwise velocity fluctuations. Lines : : mean streamwise velocity from DREAM ${ }^{\circledR}$ simulations (300x120x60); -----: rms of streamwise velocity fluctuations from DREAM ${ }^{\circledR}$ simulations $(300 \times 120 \times 60)$.

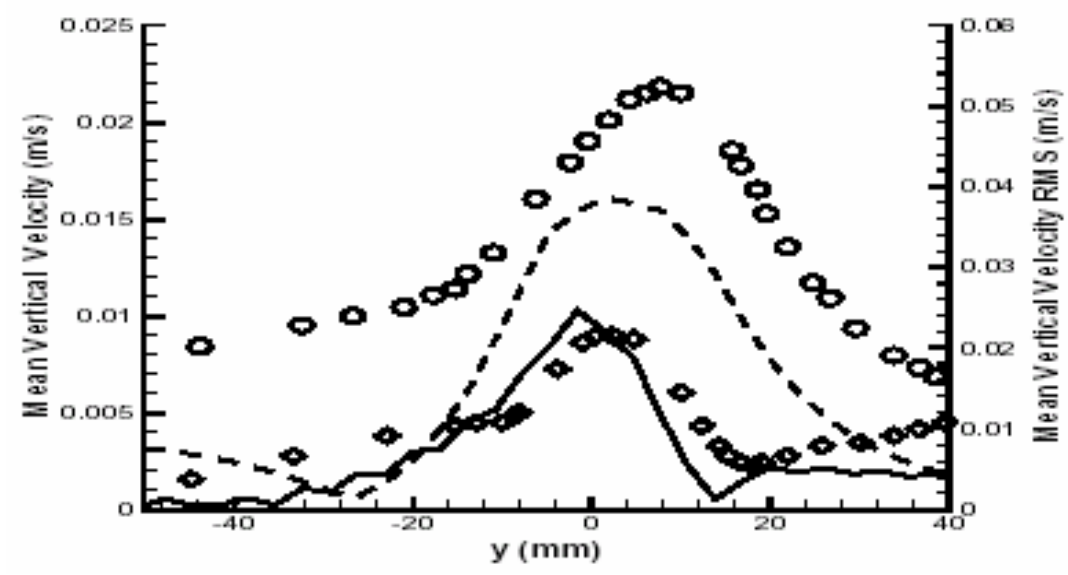

Figure 4.3.11 - Mean and rms vertical velocity at $x / \lambda=1.25$. Symbols are from Rightley (1995): $\diamond$ : mean vertical velocity; 0 : $\mathbf{r m s}$ of vertical velocity fluctuations. Lines from Shi (2001): : mean vertical velocity; -----: rms of vertical velocity fluctuations. 


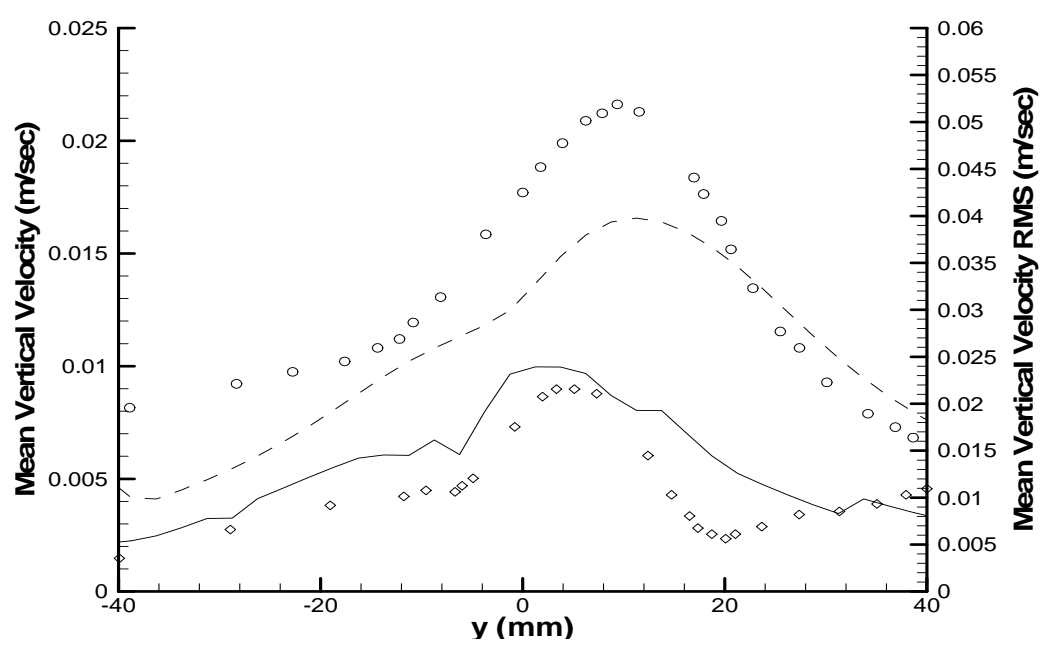

Figure 4.3.12 - Mean and $r m s$ vertical velocity at $x / \lambda=1.25$. Symbols are from Rightley (1995): $\diamond$ : mean vertical velocity; 0 : $\mathbf{r m s}$ of vertical velocity fluctuations. Lines from DREAM ${ }^{\circledR}$ Simulations: : mean vertical velocity $(200 \times 80 \times 40)$; ----: rms of vertical velocity fluctuations $(200 \times 80 \times 40)$.

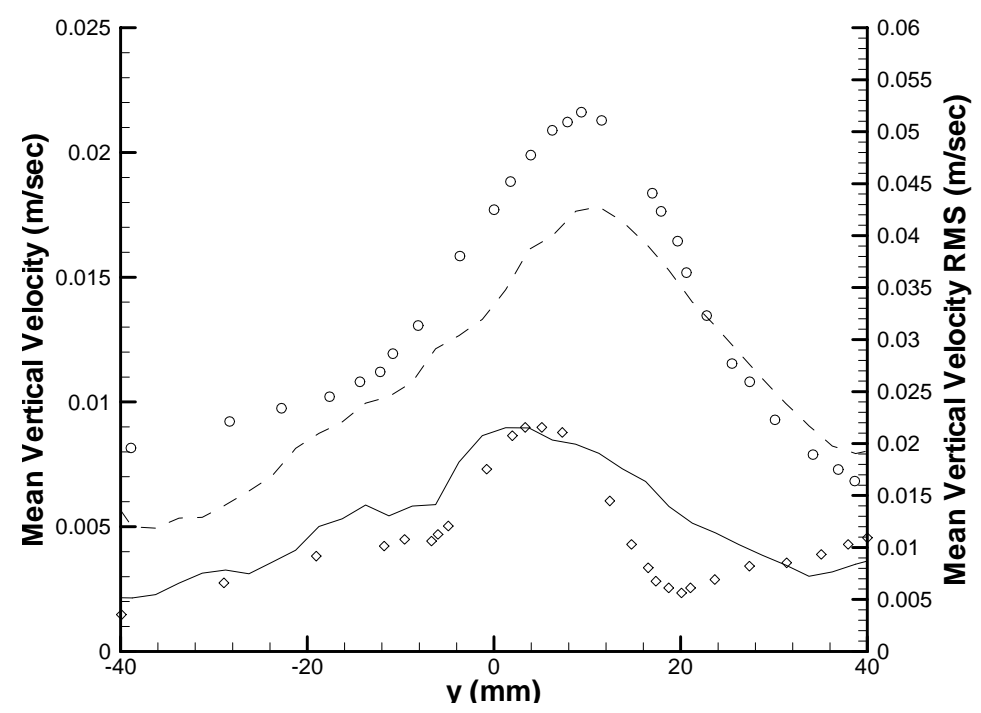

Figure 4.3.13 - Mean and rms vertical velocity at $x / \lambda=1.25$. Symbols are from Rightley (1995): $\diamond$ : mean vertical velocity; $0: \mathrm{rms}$ of vertical velocity fluctuations. Lines from DREAM ${ }^{\circledR}$ Simulations: ___ _ mean vertical velocity (300x120x40); ----: rms of vertical velocity fluctuations $(300 \times 120 \times 60)$. 


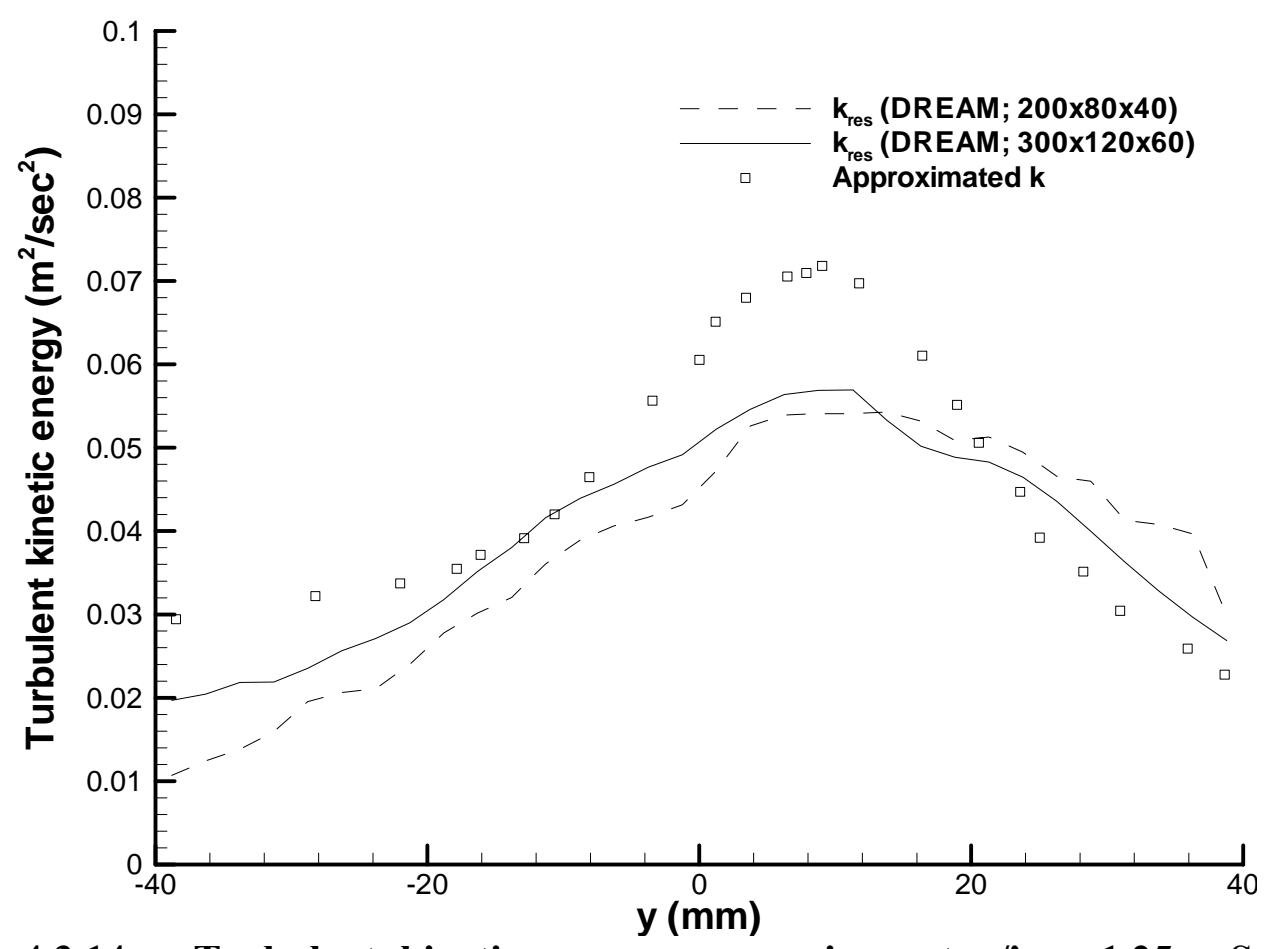

Figure 4.3.14 - Turbulent kinetic energy comparison at $x / \lambda=1.25$. Symbols represent approximation from Rightley (1995) rms data: $\square$; ---- Dashed lines represent resolved portion of turbulent kinetic energy from DREAM simulations for 200x80x40 grid; Solid lines representing only the resolved portion of the turbulent kinetic energy from the DREAM ${ }^{\circledR}$ simulations for 300x120x60 grid size. Resolved portion is approximately $85 \%$ of experimental data, which is good for LES simulations. 


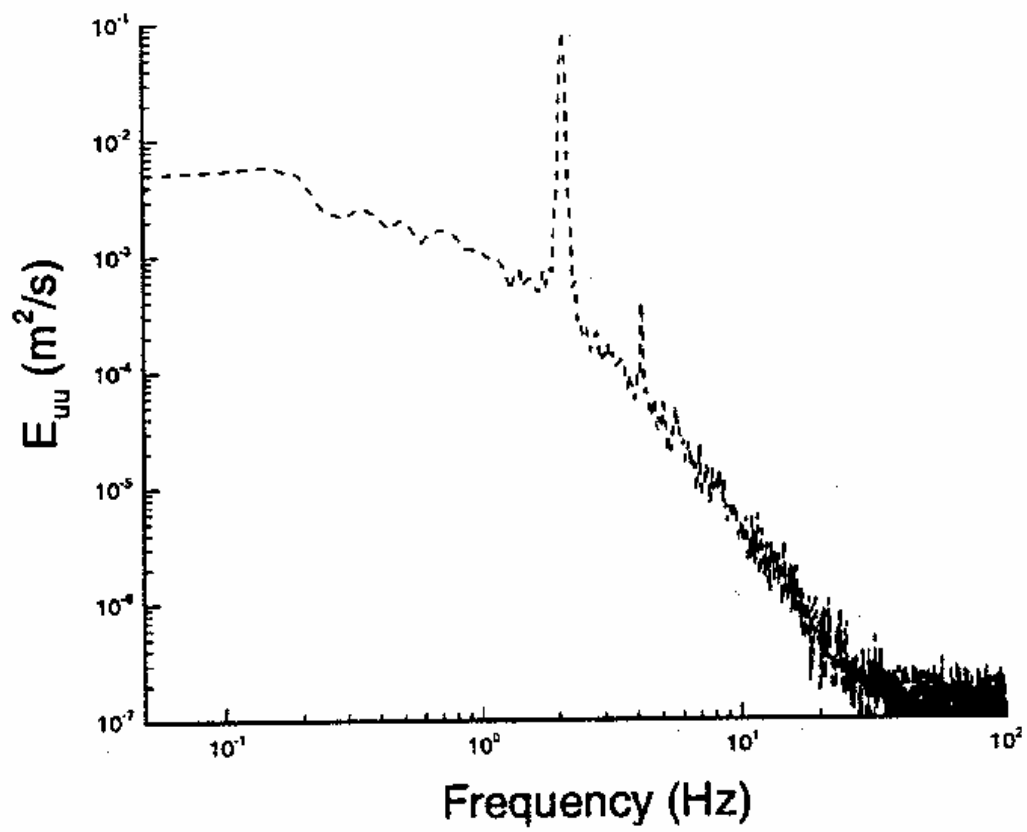

a)

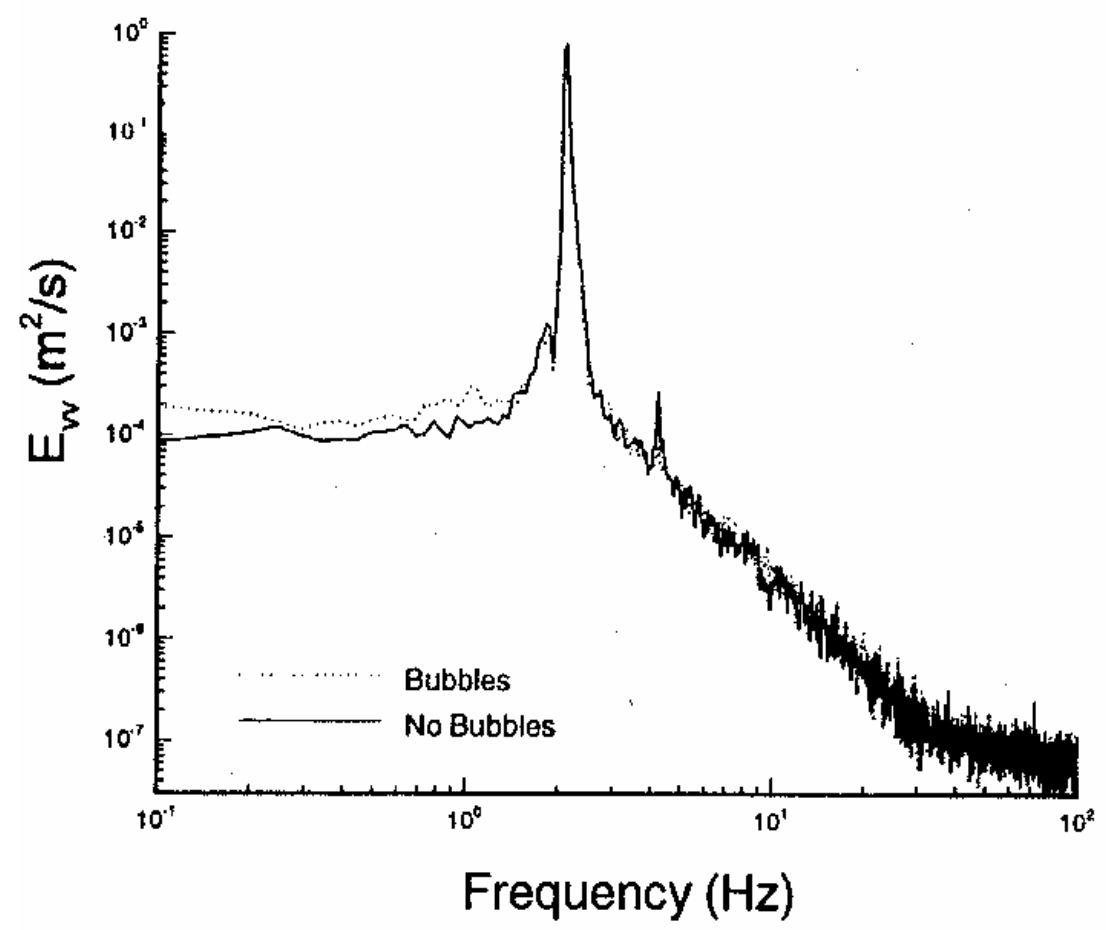

b)

Figure 4.3.15 - Energy spectra for the a) streamwise and b) vertical velocity components at $x / \lambda=1.25$ (Rightley, 1995). 


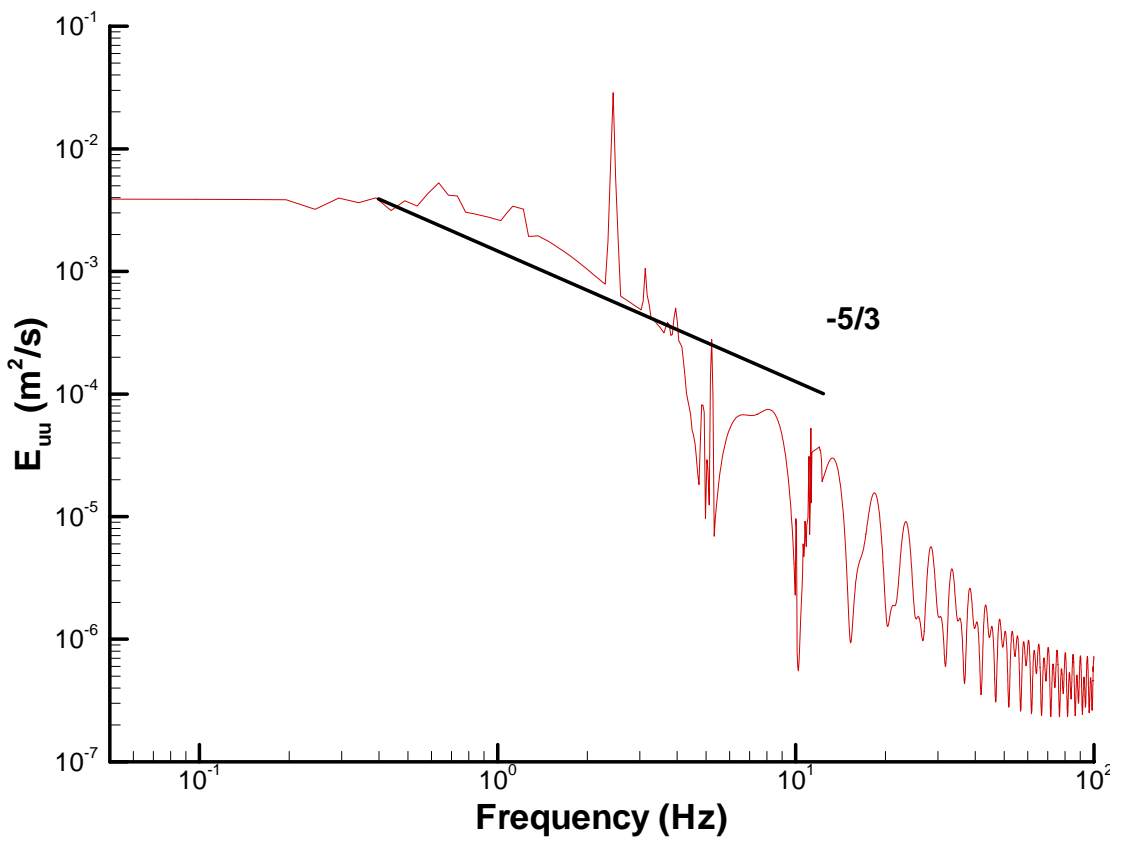

a)

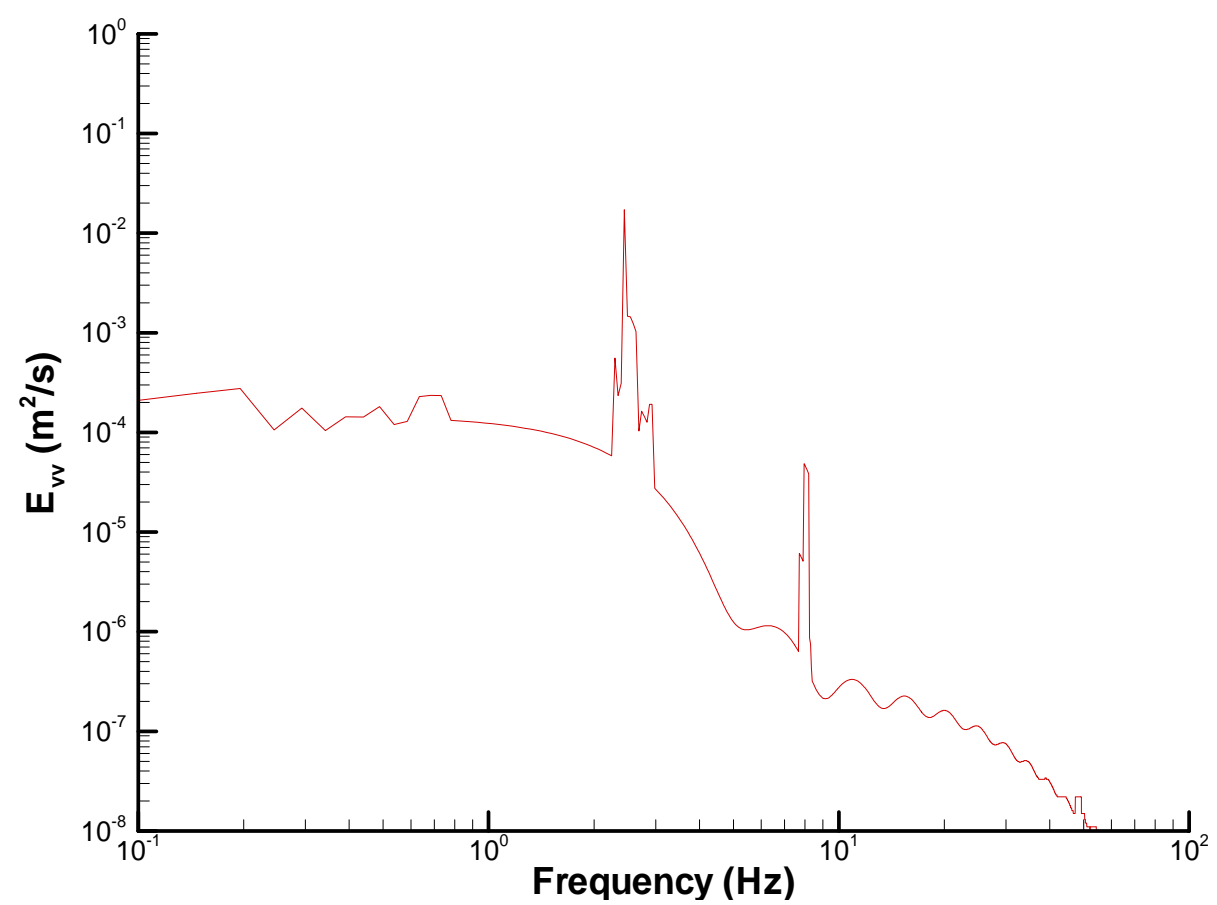

Figure 4.3.16 - Averaged energy spectra for the a) streamwise and b) vertical velocity components at $x / \lambda=1.25$ from $300 \times 120 \times 60$ DREAM $^{\circledR}$ simulations. 


\subsection{VERTICAL BUOYANT JET}

\subsection{Introduction}

The impingement of a vertically directed buoyant jet on a sharp density, stably stratified interface occurs often in nature. When a vertically directed higher density, buoyant jet impinges on a lower density interface, momentum from the jet will cause the development of a cavity. The magnitude of this cavity is governed by the Richardson number, or inverse densimetric Froude number, which is dependent on the jet inlet and buoyancy differences between the fluids. The initial development of the cavity is distinct and symmetric; however, over time, the effects of instabilities lead to a non-symmetric turbulent flow field. The behavior of the ensuing jet, as well as an investigation into the characteristics of the flow field is performed in this chapter using LES and ITM methods.

\subsection{Vertical Buoyant Jet Impingement}

Friedman et. al. (2001) determined from experiments performed at The Johns Hopkins University, that for the vertical buoyant jet impingement generally, three regimes could be characterized using a Richardson number analysis. The resulting descriptions were based on the amount of entrainment into the upper layer by the jet, coupled with the size of the cavity formed from the impinging jet. A schematic of the experimental facility is shown in Figure 5.1.a.

The fuel layer is contained in the upper center portion of the tank by two fuel weirs, and the water enters the tank through the vertical inlet pipe. In all cases simulated, which are presented in Section 5.3, only diesel fuel is considered, whereas some experiments used SAE-oil. As the water penetrates the fuel layer, mixing will occur if the shear forces are great enough compared with the buoyancy, or gravity, forces (i.e. if the densimetric Froude number is large or Richardson number is low). As the water becomes entrained in the fuel layer, the excess water is forced to the sides of the tank and exits through the drains located in the upper left and right compartments. If no mixing occurs, then the excess fuel will be forced over the fuel weir where it can return to the fuel supply. An impingement plate is located in the center of the tank above the inlet pipe. If the velocity 
of the incoming water is large enough, then the jet will impinge on the plate. This will cause breakage of the inlet jet and the formation of fuel and water droplets, which will cascade back toward the fuel/water interface. For the simulations presented in this study, wall effects are excluded and there are no plate effects. However, the curved buoyant jet simulations presented in the next chapter do impinge at the wall, and these can be compared qualitatively with some experiments by Friedman et. al. (1999).

As this study is focusing on the performance of DREAM $^{\circledR}$ using ITM LES methods for variable density flows, only simulations which are fully turbulent will be explored. Thus, the Richardson number will be less than 2.5 for all simulations, which are in flow regimes two or three as indicated by Friedman et. al. (1999). The Richardson number, as define by Friedman et. al. (1999) is

$$
R i=\frac{g \Delta \rho}{\rho_{w}} \frac{D_{i n}}{U_{i n}^{2}}
$$

Where $\mathrm{g}$ is the gravitational acceleration, $\rho$ is the density, $\mathrm{D}$ is the inlet jet diameter, and $\mathrm{U}$ is the inlet velocity. Thus, a distinct cavity will be formed with high density gradients and these "plumes" become unstable. After impingement on the fuel interface, the water collapses due to turbulence and buoyancy. Friedman et. al. (1999) describes that after the collision, fuel will then extend below the initial interface. Due to the high degree of momentum of the jet, a fuel - water mixing region is created below the interface as the fuel is pushed out of the initial zones. For higher inlet jet velocities, the dispersion after impact may result in cloud as it again descends due to buoyancy. As the performance of DREAM $^{\circledR}$ in the turbulent jet regions is the primary concern, modifications to the calculation domain have been made. These modifications are further explained in the next section.

\subsection{DREAM ${ }^{\circledR}$ Simulation Details}

The water jet - fuel layer interface resulting from impingement is investigated in this section. The computational domain is modeled as a three dimensional tank, with dimensions of $0.4 \mathrm{~m} \times 1.0 \mathrm{~m} \times 1.0 \mathrm{~m}$. These dimensions are identical to the experimental test facility used by Friedman et. al. (1999) in the vertical and spanwise direction, but 
differs in the streamwise direction because no impinging plate is included. No plate or baffles are included in the geometry, and the interface is kept constant through use of appropriate inlet and outlet boundary conditions of the fuel and water. This ensures that no wall effects are felt by the jet in the region of interest, $0.4<\mathrm{y}<0.6 \mathrm{~m}$, as the flow behavior around the baffles is of no concern in this study. Constant pipe dimensions were used for all simulations, being 0.10 meters high with a radius of 0.014 meters, which is 0.05 meters short of the fuel layer. A cross-plane schematic of the geometry used in the simulations is shown in Figure 5.1.b. Table 5.3.1 shows the computational parameters used to achieve the appropriate experimental Richardson number similarity. A total of six different Richardson number simulations were performed, with 0.08 and 2.5 being the range of Richardson numbers studied. A Richardson number of 2.5 is the lower boundary of regime 2 as described in Friedman et. al. (1999).

It is important to note that the goal of this chapter is to determine two capabilities of DREAM $^{\circledR}$; being 1) the ability of ITM methods, as implemented into DREAM ${ }^{\circledR}$, to resolve the small scales and predict appropriate amounts of turbulent kinetic energy within the flow, and 2) test the ability of DREAM ${ }^{\circledR}$ to capture the large scale instabilities of the buoyant jet. Therefore, two different grids are used in the simulations, being a coarse grid of 50x157x157 and a fine grid of $100 \times 213 \times 213$, yielding 1.2 and 4.5 million grid cells. A time step of 0.001 seconds is used for all simulations, which was determined through numerical experimentation to yield an appropriate solution as compared to the experiments. A constant fuel droplet size of $1.5 \mathrm{~mm}$ is used in the slip velocity relationship, as suggested by Friedman et. al. (1999). For more information on the slip velocity relationship as used in $\operatorname{DREAM}^{\circledR}$, the reader is referred to Kandil (2001). The bulk jet flow is in the positive $\mathrm{x}$-direction and the momentum equations are adapted accordingly, meaning that gravity is now applied in the negative $\mathrm{x}$ direction. This treatment is common in many CFD simulations, and is identical to a vertical buoyant jet with gravity in the negative $y$ direction. In effect, $x$ is the vertical direction. The results are identical; however, for visualization and comparative purposes, the results are presented in the same coordinate system.

For the simulations in this section, some non-uniformity in the grid is used, which allows for enough grid resolution within the important regions of the flow, being the inlet 
pipe and interface locations. For the coarse grid simulations, 60 grid cells are within 1.5 inlet pipe diameters and 22 are located near the fuel-water interface. The fine grid simulations had 109 and 82 cells in the respective locations. Although using a nonuniform grid does introduce some error in the calculations, it is believed to be small and should not intrude on the overall quality of the simulations. As cylindrical coordinates are not implemented into the code, great care of creating a pipe must be taken. The circular pipe geometry is achieved through manually "blocking out" appropriate cells outside of the desired diameter, which results in a step wise circle with walls 1 cell thick. Details of this technique may be found in Appendix B, Section B.2.

\begin{tabular}{|l|l|l|l|l|}
\hline Fluid & D (m) & Fr & Ri & $\mathbf{U}_{\text {in, sims }}(\mathbf{m} / \mathbf{s e c})$ \\
\hline Diesel - water & 0.014 & 0.4 & 2.5 & 0.110 \\
\hline Diesel - water & 0.014 & 0.9090 & 1.1 & 0.228 \\
\hline Diesel - water & 0.014 & 1.754 & 0.57 & 0.475 \\
\hline Diesel - water & 0.014 & 4.762 & 0.21 & 1.29 \\
\hline Diesel - water & 0.014 & 8.33 & 0.12 & 2.26 \\
\hline Diesel - water & 0.014 & 12.5 & 0.08 & 3.39 \\
\hline
\end{tabular}

Table 5.3.1 - Simulation parameters from buoyant jet experiments from Friedman et. al. (1999).

\subsection{Results and Discussion}

If URANS, Unsteady Reynolds Averaged Navier-Stokes, simulations were performed for this type of flow, such as those studied by Kandil (2001), an appropriate turbulence model capable of handling re-laminarization would be necessary. Also, it is very unlikely that the URANS simulations would capture any type of jet or flow field instabilities. It is these types of flows that are highly suitable for LES and ITM type methodologies. 
The ability of DREAM ${ }^{\circledR}$ to accurately solve the complex problem of impingement of a water jet onto a fuel layer, will help to ensure that both the ITM and variable density implementation is accurate and appropriate for more complex turbulent, buoyant flows. In the experiments, as the water leaves the inlet pipe and enters the fuel layer, the maximum penetration depth of the jet is based on the amount of momentum present within the water jet exiting the pipe. Experimental results are shown in Figure 5.2 for Ri $=0.08$, with the corresponding coarse and fine grid density contours from the DREAM ${ }^{\circledR}$ simulations presented in Figures 5.3 and 5.4. Only the fine grid and Richardson number equaling 0.08 are presented for brevity, as well as the fact that higher Richardson numbers also exhibit similar trends, but only less in magnitude.

All simulations, even using a coarser grid, were able to accurately predict the maximum penetration depth based on the Richardson number as compared to experiments. This is to be expected while penetration depth is governed by integral conservation of the mass and momentum, which is always fulfilled in the simulations. A summary of the non-dimensional jet penetration depth, $A R=h_{\text {jet }} / D_{p}$, are shown in Figure 5.5 .

As the jet reaches its apex and loses momentum, buoyancy forces cause the water jet to fall, and instabilities arise. This phenomenon is apparent for all simulations that are turbulent, although the amount of instability is dependent on the Richardson number. The onset of the instabilities takes place at about 1.2 seconds for $\mathrm{Ri}=0.08$ as shown in Figure 5.6, with the DREAM ${ }^{\circledR}$ simulations predicting this onset at 1.22 and 1.205 seconds for the coarse and fine grid simulations, shown by instantaneous density contours in Figure 5.6b and 5.6c.

Although the density profiles accurately predict the behavior of the experiments, little information on the flow field may be extracted from these. Thus, to determine the robustness of the DREAM ${ }^{\circledR}$ simulations, an analysis of the flow field characteristics is presented. The maximum impingement of the water jet for $\mathrm{Ri}=0.08$ is approximately 0.05 meters into the fuel layer, which again decreases as the Richardson number is increased. Two different axial locations near the interface have been chosen for analysis, being the center of the inlet pipe, and 2.5 radii from the center. Data across the jet was also analyzed at 0.01 meters below the maximum impingement depth and are also 
presented for $\mathrm{Ri}=0.08$. This location was chosen to capture any fluctuations across the unstable jet. It is important to note that similarity analysis of the buoyant jet is not applicable for these simulations, as the jet never becomes fully developed.

One of the ways to determine the amount of turbulence present within the flow field is through analysis of the instantaneous vorticity contours, which have been normalized, shown in Figures 5.7 and 5.8, for $\mathrm{Ri}=0.08$. In both the fine grid and coarse grid simulations, the largest amount of vorticity is near the interface of the mixing region. As the instability of the jet begins to dominate the flow, the vorticity increases near the interface and decreases away from the jet. The r.m.s velocity of the jet for the fine grid displays a similar property seen in many similarity type flows, whereby the r.m.s velocity is approximately twenty-five percent of the mean velocity at the centerline, shown in Figure 5.9. The r.m.s velocity decreases by approximately $300 \%$ at 0.025 meters away from the centerline (see Figure 5.10) on the average, and tends to zero further away from the centerline. A strong anisotropy is apparent between the axial and the radial directions. The difference between the r.m.s. values in the $\mathrm{y}$ and $\mathrm{z}$ directions must be due to taking insufficiently long time averaging. A spatial averaging in the radial direction may be needed. The asymmetry in these profiles are also the artifact of short time averaging without space averaging.

For the fine grid simulations, the resolved turbulent kinetic energy at the centerline and $0.025 \mathrm{~m}$ away from the centerline, which corresponds to the edge of the cavity, are shown in Figures 5.11. Again, the maximum resolved turbulent kinetic energy is at the centerline of the impinging jet. For all simulations, it must be noted that the resolved turbulent kinetic energy from the coarse grid simulations were less in magnitude than the fine grid simulations by approximately five percent, which indicates that a nearly grid independent solution is obtained. Figures 5.12 and 5.13 shows the energy spectrum at the two different locations. Again, as with the other simulations presented in Chapter 4, the calculated energy spectrum agrees with the theoretical inertial subrange slope from Kolmogorov's theory. This indicates that the simulations are sufficiently accurate. Also, as the data is examined away from the jet, the maximum of spectra decreases by about a factor of ten for each $0.025 \mathrm{~m}$, which indicate that the length scales decrease within the same region. A similar type of trend is observed as the Richardson number is decreased. 
The behavior of the jet from the radius of the jet outward, at a constant height, is briefly examined as well. From Figures 5.17 and 5.18, the turbulent intensity and resolved turbulent kinetic energy clearly decrease away from the centerline. Again, the asymmetry is most likely due to the short time averaging of the data.

Thus, in conclusion, the accuracy of the DREAM simulations shows the validity and capability of DREAM to simulate these types of flows. Insight is gained through analysis of the turbulence quantities, and indicates that the turbulence decreases away form the centerline. A similar trend is observed for all of the Richardson numbers simulated; however, the overall magnitude of each of the quantities does in fact decrease. This clearly shows that as long as an appropriate grid resolution is maintained within the region of interest, the important physics of the buoyant turbulent flow field may be obtained. However, a decrease in the time step will enhance the ability to predict the resolvable turbulence within the flow. Therefore, this section demonstrated the ability of DREAM $^{\circledR}$ to predict variable density, turbulent flows using LES and ITM type methods. 


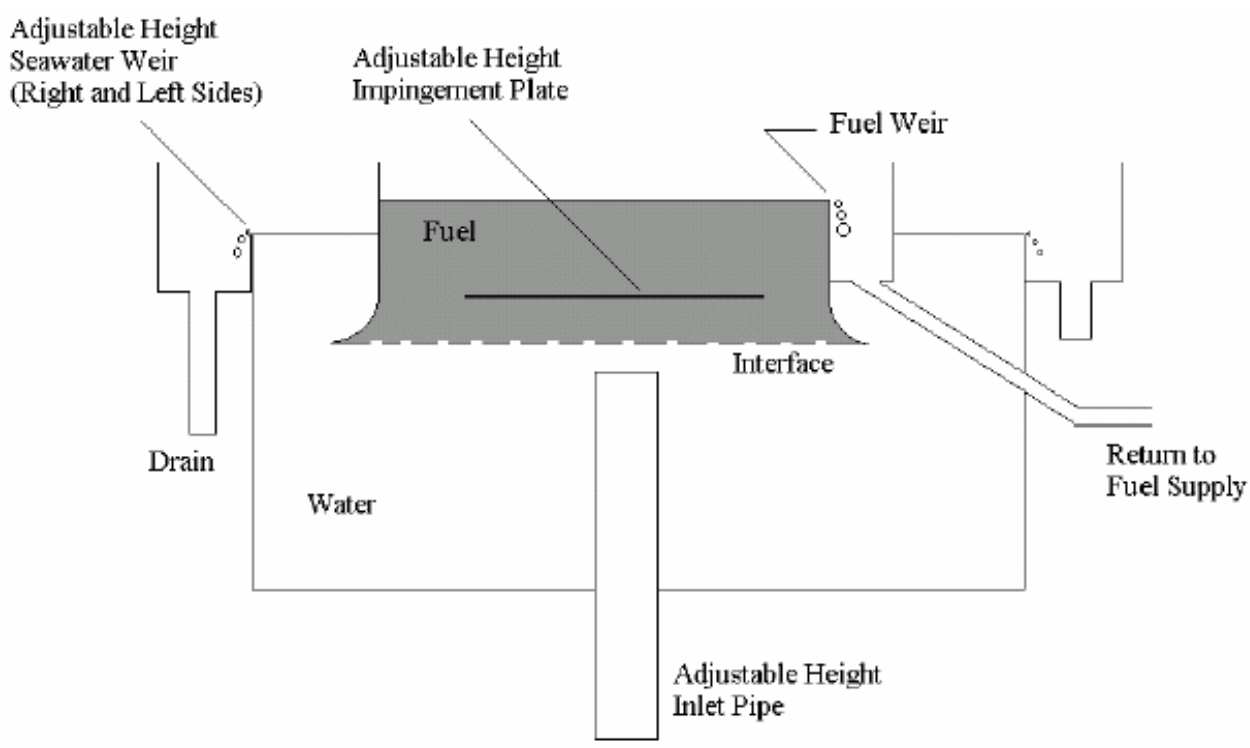

Figure 5.1.a - Experimental fuel impingement test facility used at The Johns Hopkins University by Friedman and Katz (1999).

Center plane view of geometry used in simulations

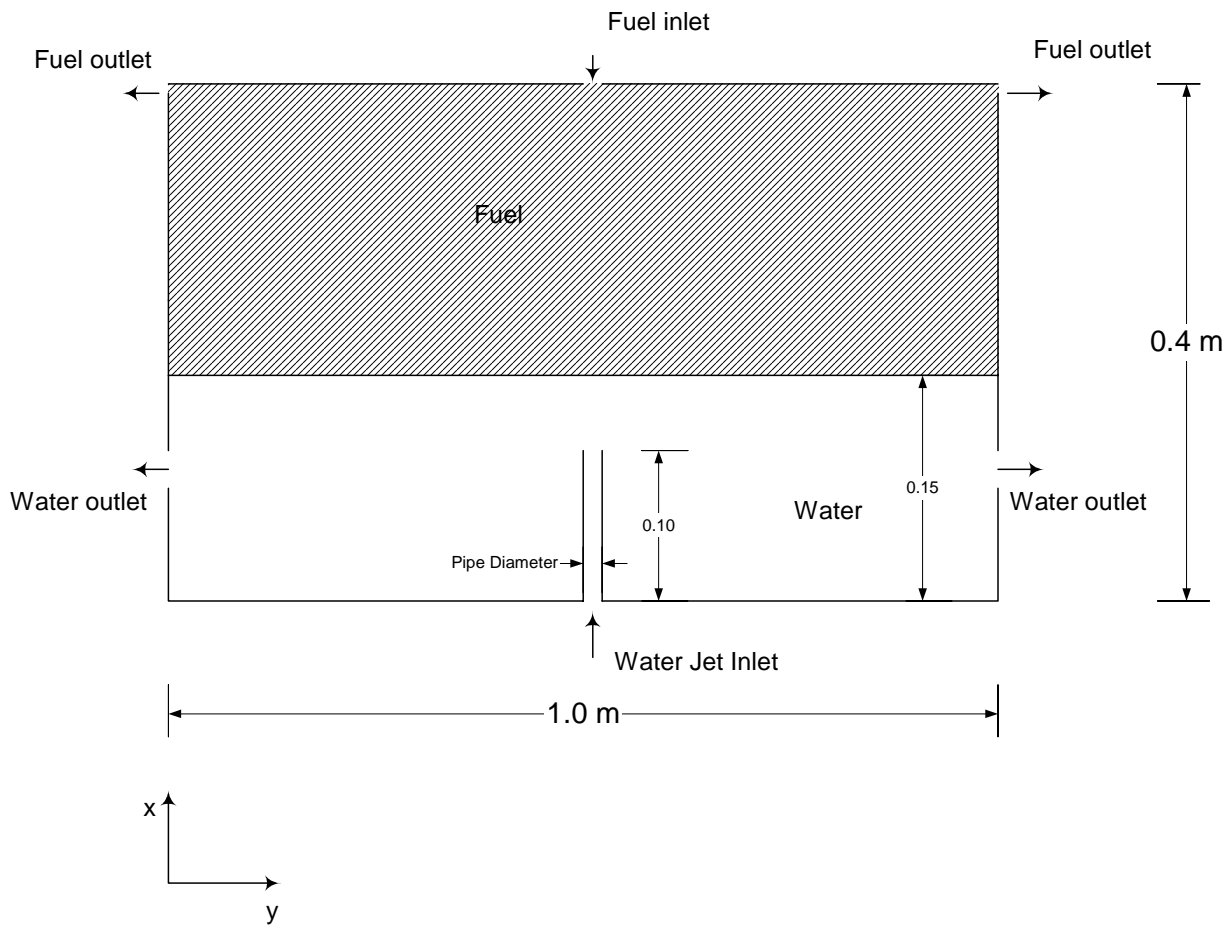

Figure 5.1.b - Center plane view of the geometry used in the DREAM ${ }^{\circledR}$ simulations 


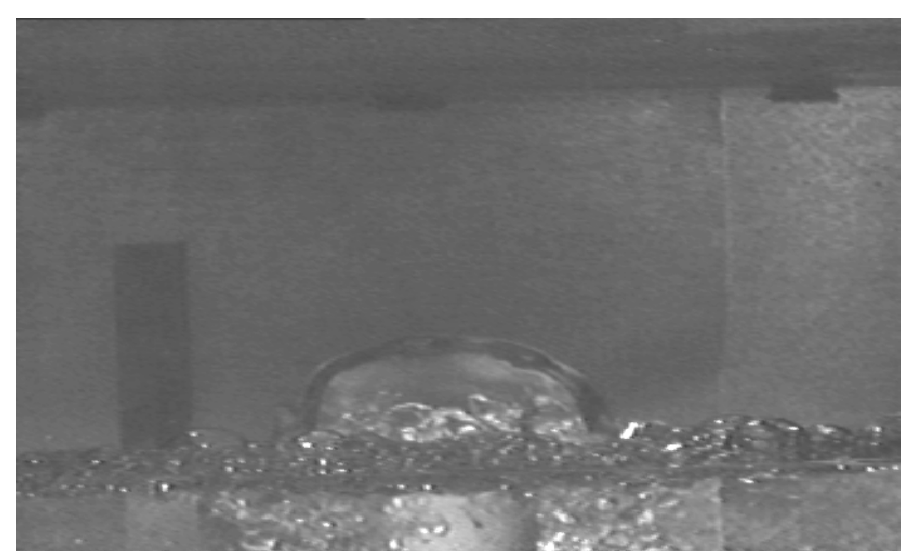

Figure 5.2 - Experimental impinging jet instantaneous picture for Regime 3 showing initial water jet impingement onto upper fuel layer for $R i=0.08$; cavity is symmetric.

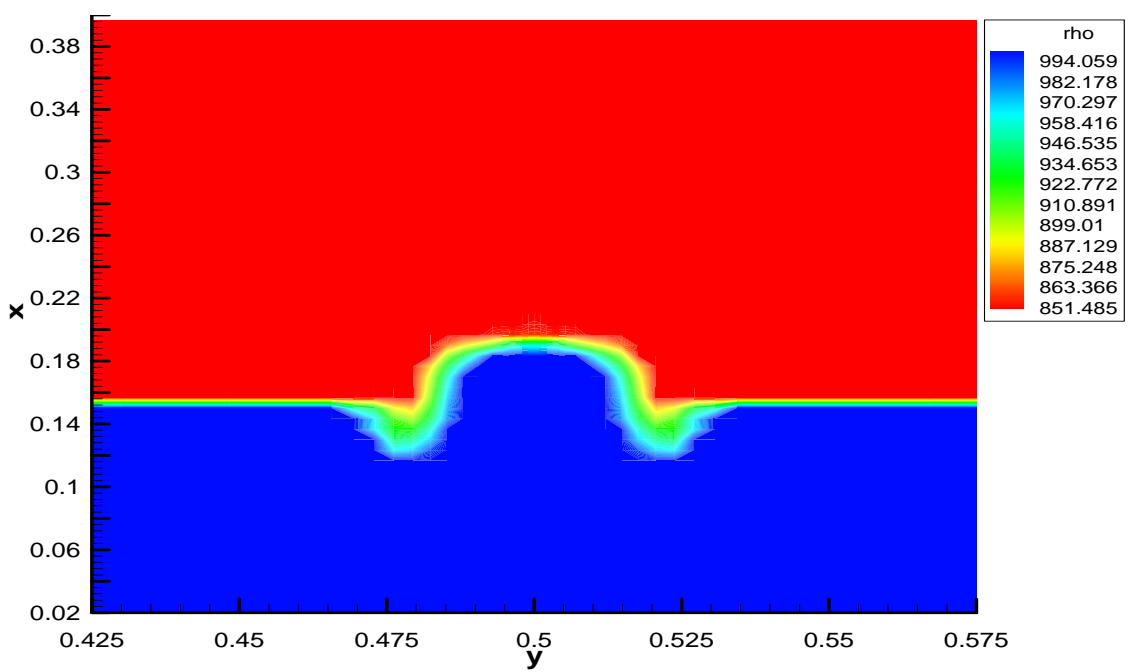

Figure 5.3 - Density contour from DREAM ${ }^{\circledR}$ simulations for $R i=0.08$ using $50 \times 157 \times 157$ grid size with $\mathrm{dt}=0.001$ seconds; $t=0.4$ seconds after impact.

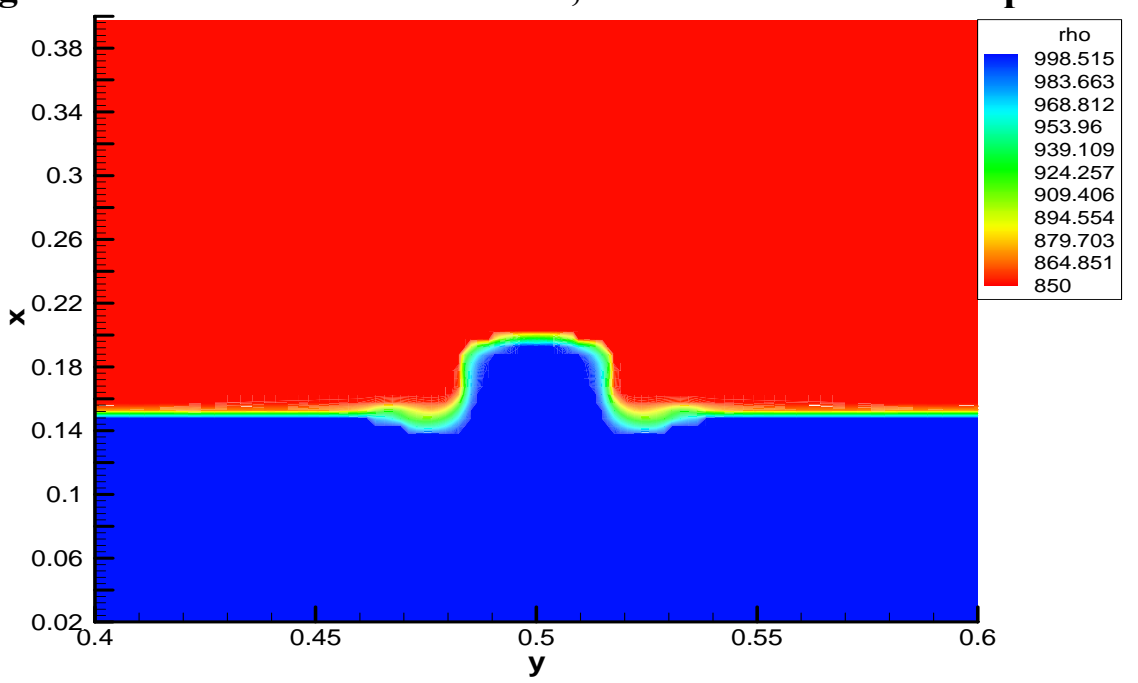

Figure 5.4 - Density contour from DREAM ${ }^{\circledR}$ simulations for $\mathrm{Ri}=\mathbf{0 . 0 8}$ using $100 \times 213 \times 213$ grid size with $d t=0.001$ seconds; $t=0.4$ seconds after impact. 


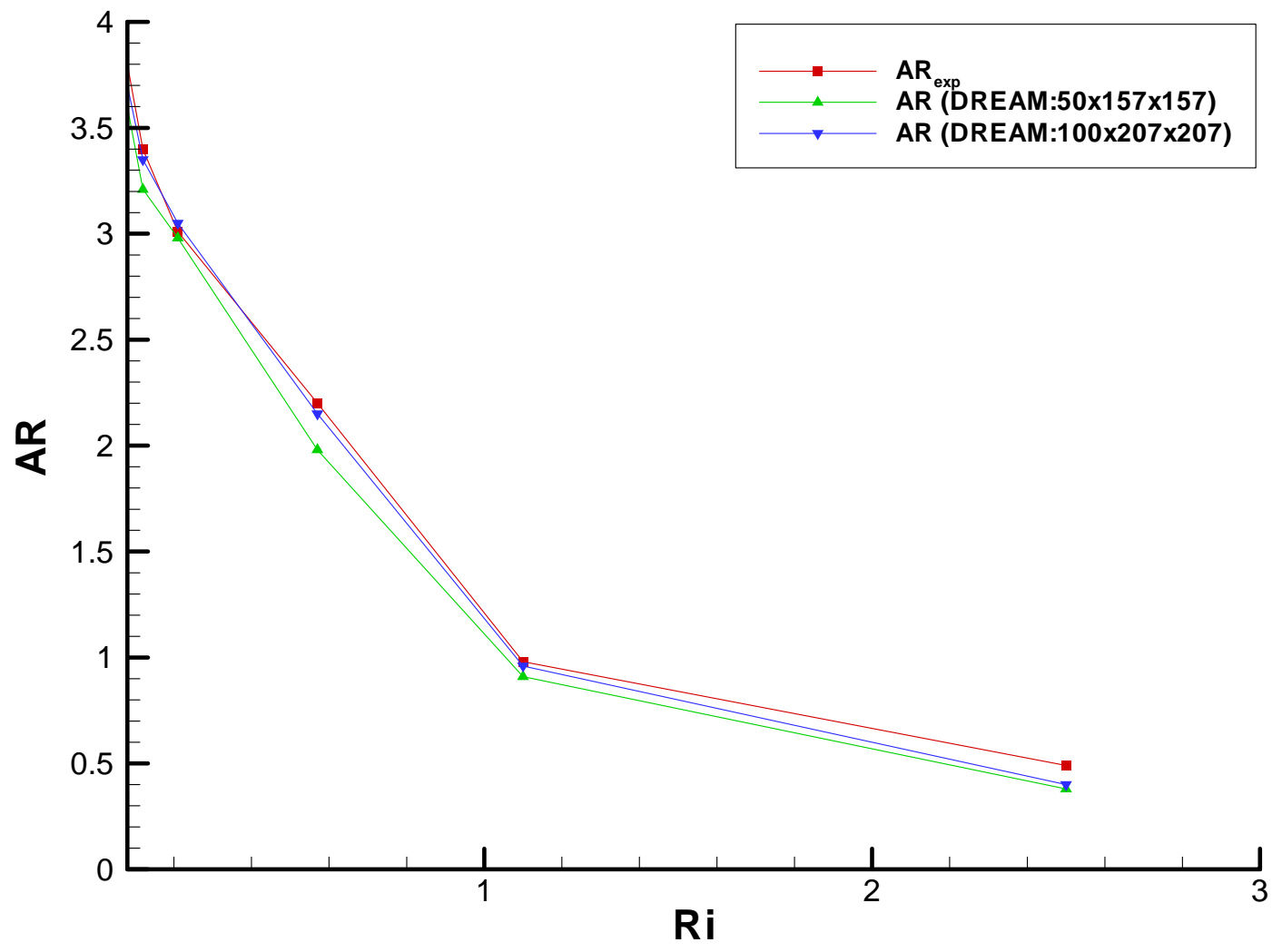

Figure 5.5 - Comparison of DREAM ${ }^{\circledR}$ simulations using 50x157x157 and $100 \times 213 \times 213$ grid sizes to experimental maximum penetration depth $(A R)$ data; $d t=$ 0.001 seconds. 


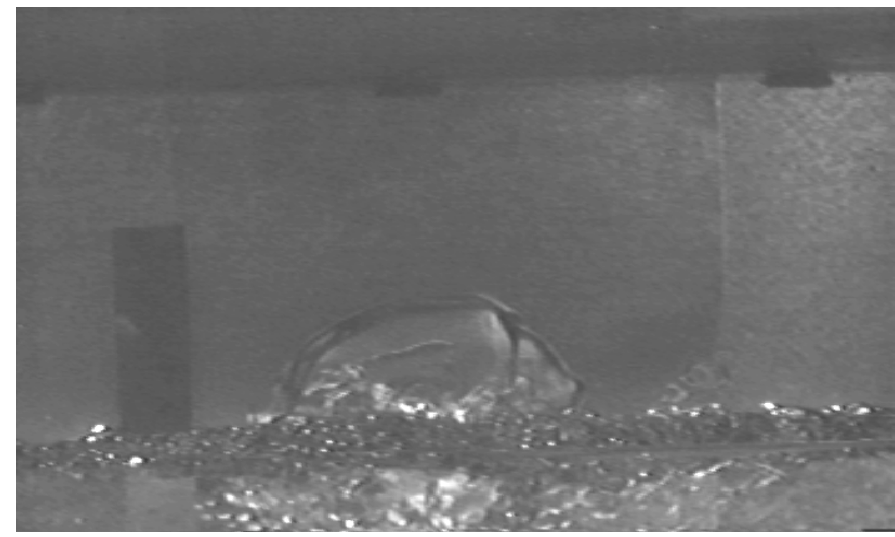

(a)

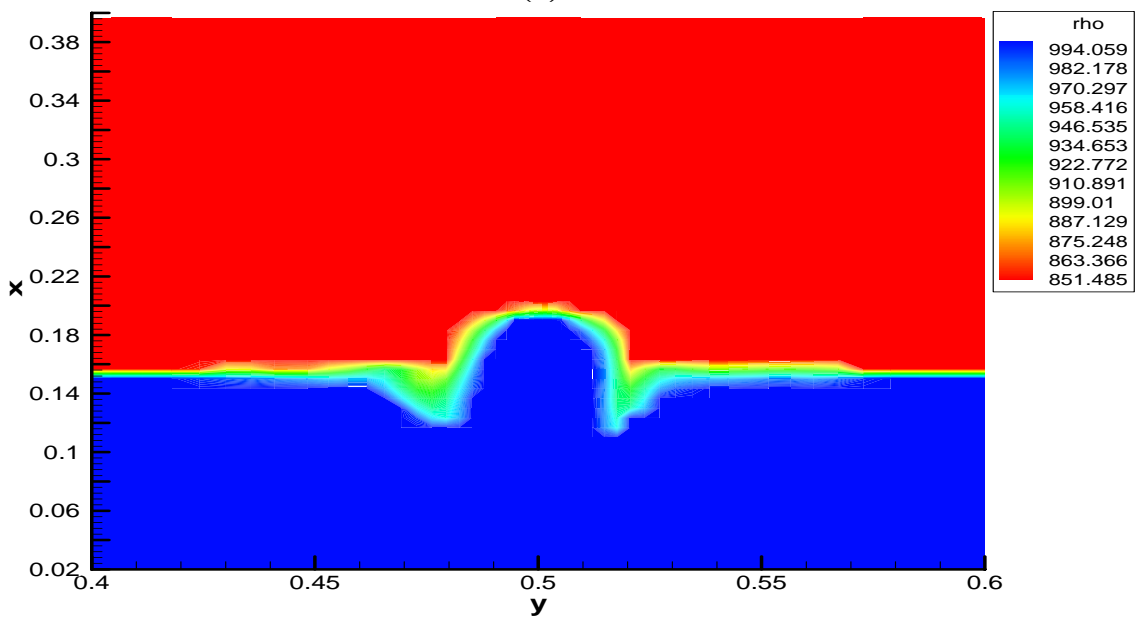

(b)

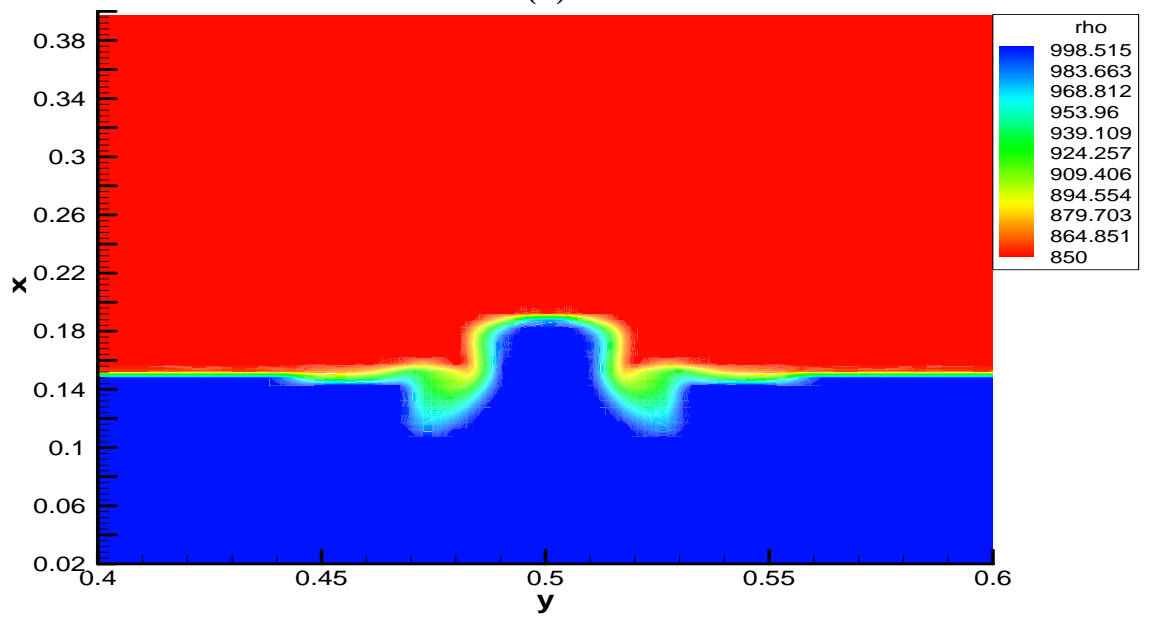

(c)

Figure 5.6 - (a) Experimental impinging jet experiment for Regime 3 showing onset of water jet instability for $R i=0.08, t=1.2$ seconds after initial jet impingement. DREAM $^{\circledR}$ simulations for $\mathrm{Ri}=\mathbf{0 . 0 8}$ using $(\mathrm{b}) 50 \times 157 \times 157, t=1.22$ seconds after initial jet impingement, and (c) $100 \times 213 \times 213$ grid size showing onset of water jet instability, $t=1.205$ seconds after initial jet impingement. 


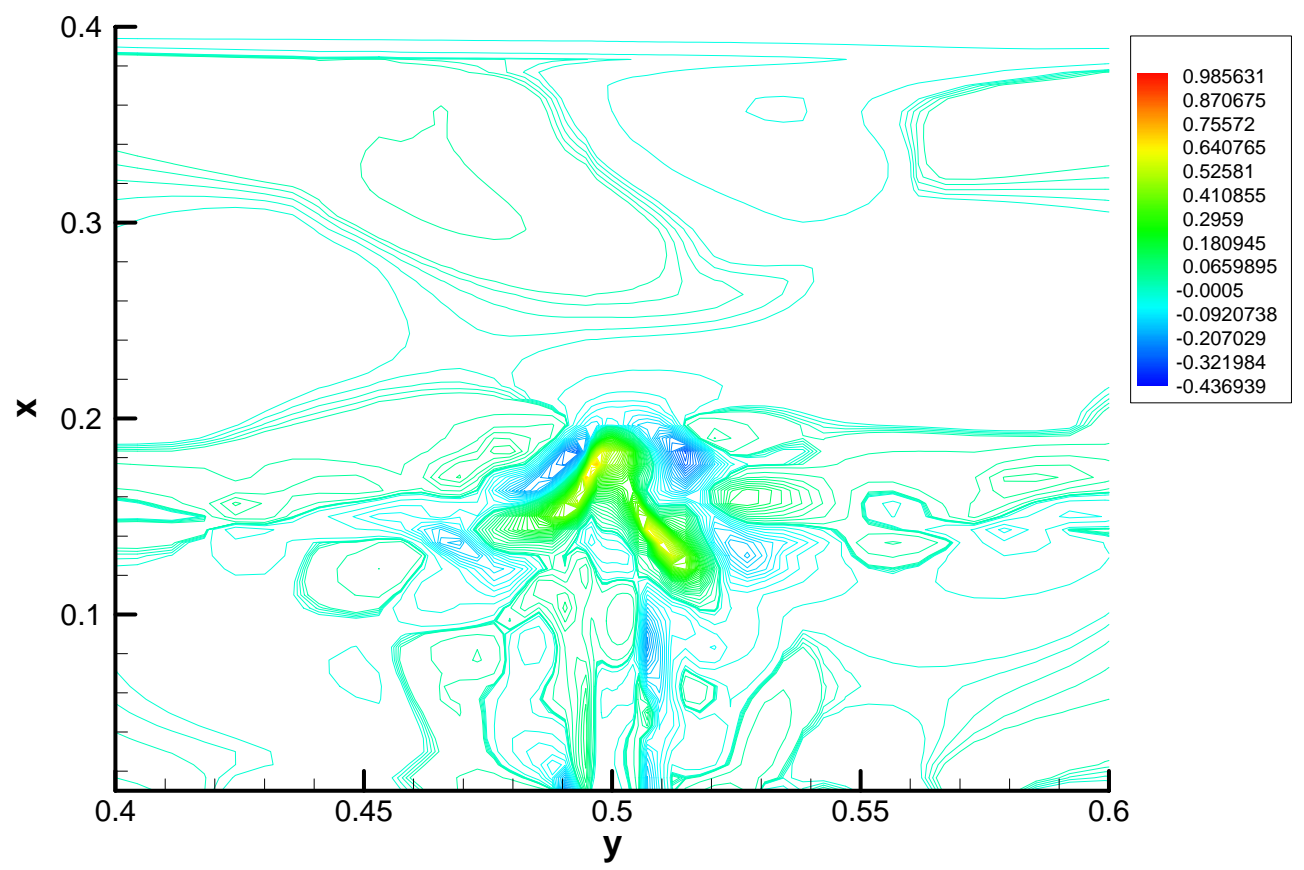

Figure 5.7 - Instantaneous normalized $z$ vorticity contours, with vectors, for $\mathbf{R i}=$ 0.08 using 50x157x157 grid size; $d t=0.001 ; t=1.2$ seconds after initial impact

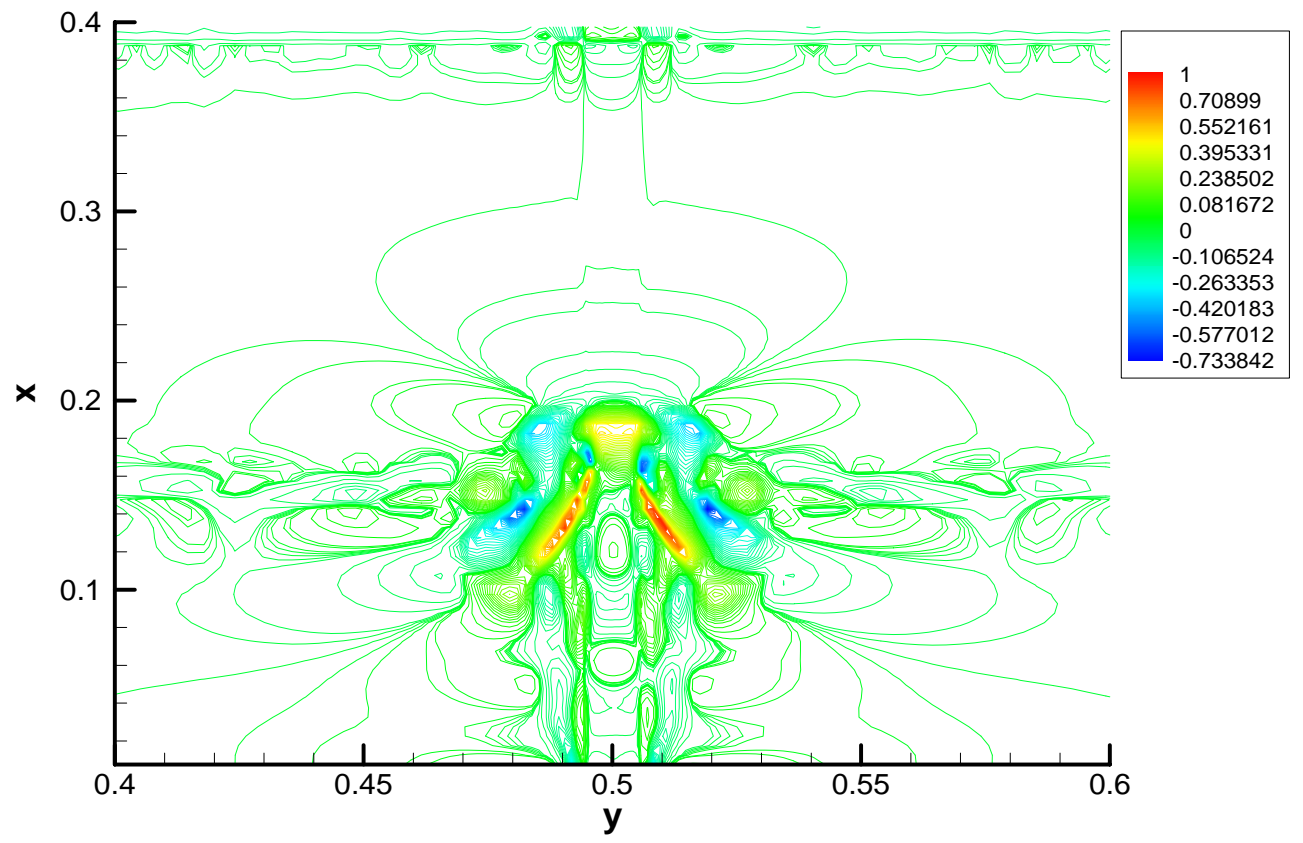

Figure 5.8 - Instantaneous normalized $\mathrm{z}$ vorticity contours, with vectors, for $\mathbf{R i}=$ 0.08 using $100 \times 207 \times 207$ grid size; $d t=0.001 ; t=1.2$ seconds after initial impact 


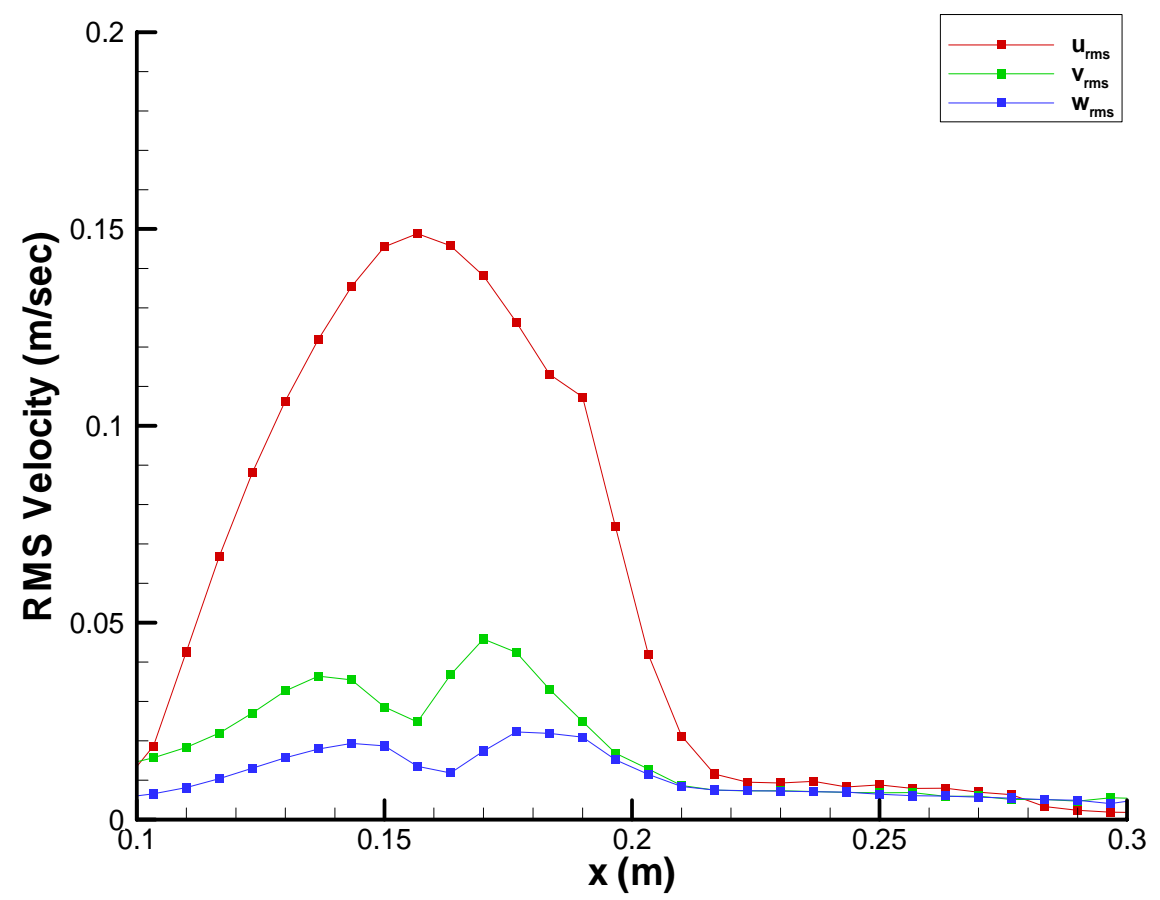

Figure 5.9 - Resolved RMS for $\mathrm{Ri}=0.08$ using $100 \times 213 \times 213$ grid size at $\mathrm{y}=0.5$; $\mathrm{dt}=$ 0.001 seconds.

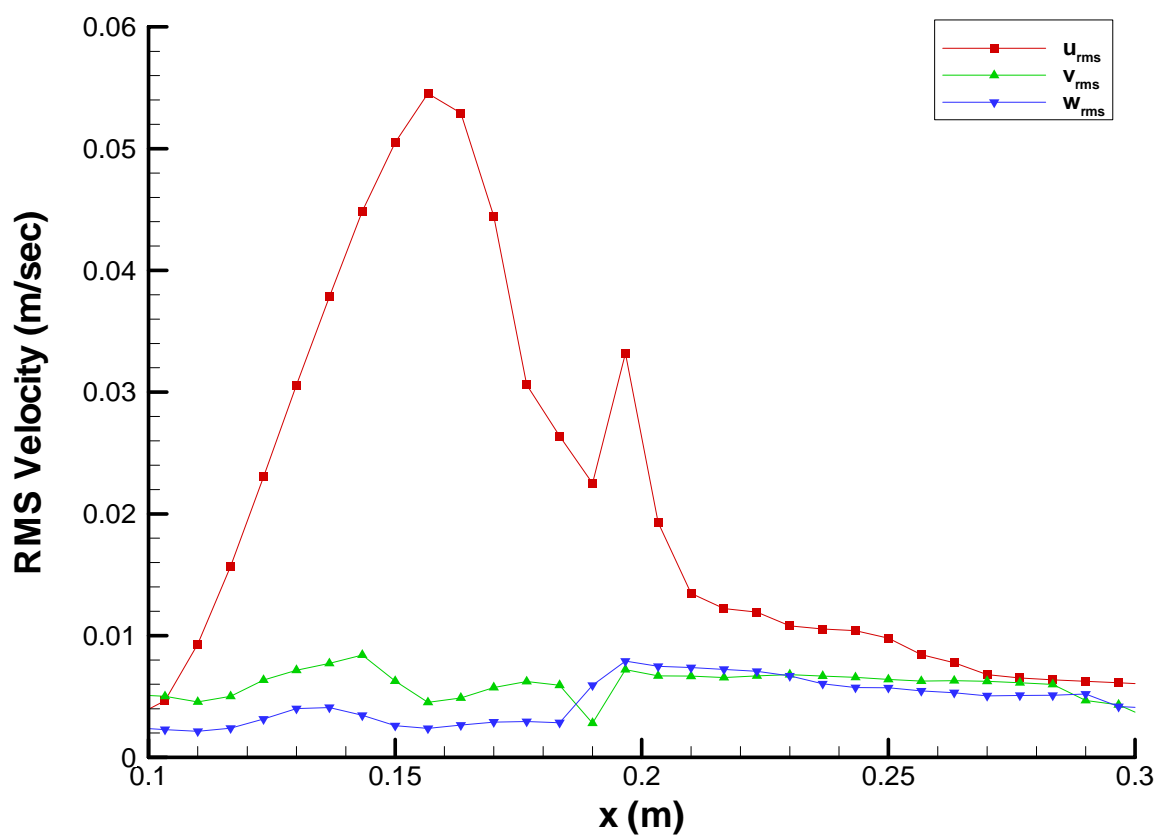

Figure 5.10 - Resolved RMS for $\mathbf{R i}=0.08$ using $100 \times 213 \times 213$ grid size at $\mathbf{y}=\mathbf{0 . 5 2 5}$; dt $=0.001$ seconds. 


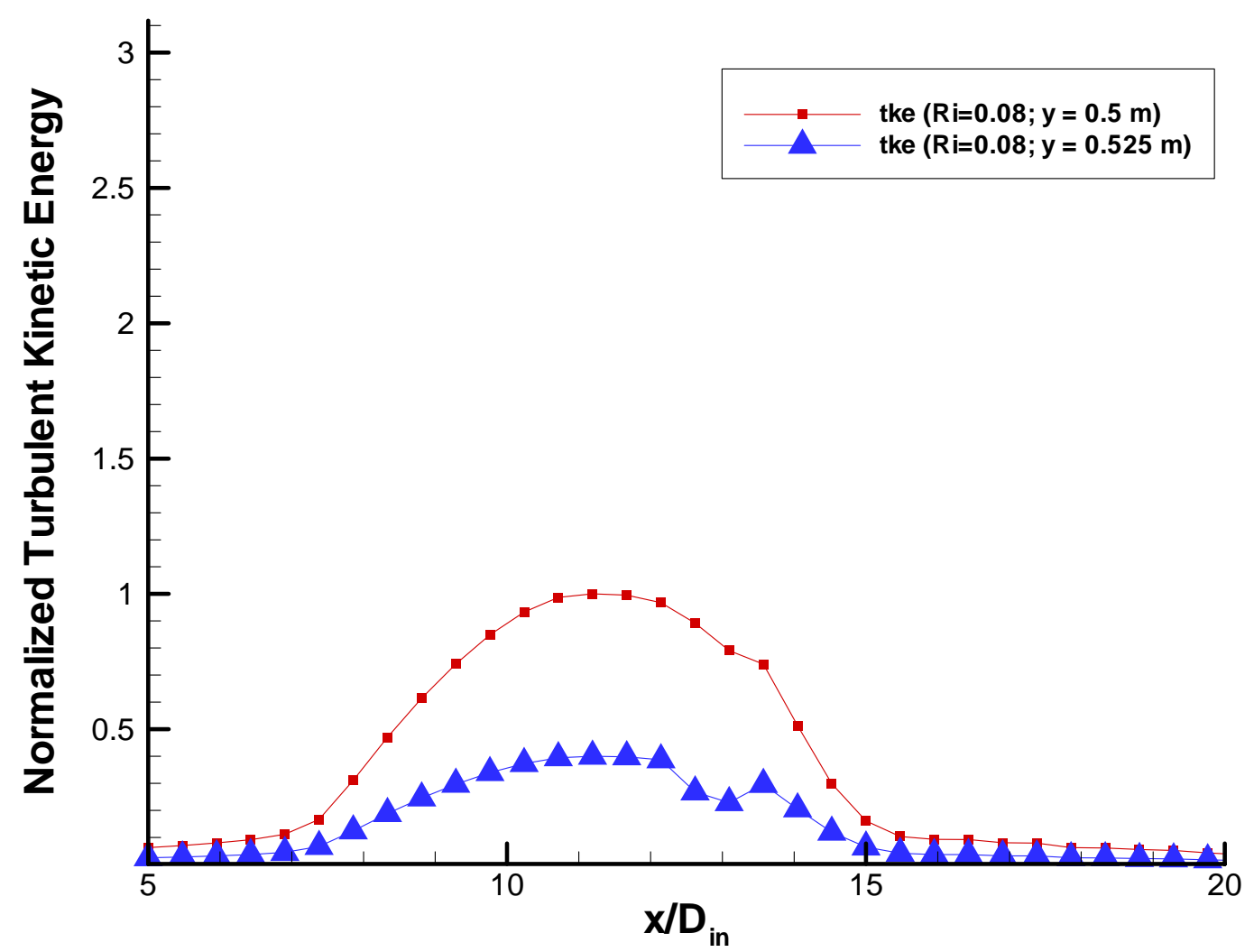

Figure 5.11 - Normalized resolved turbulent kinetic energy for $\mathbf{R i}=\mathbf{0 . 0 8}$ using $100 \times 213 \times 213$ grid size at $\mathrm{y}=0.5$ and $\mathrm{y}=0.525 ; \mathrm{dt}=0.001$ seconds. Jet inlet is located at $x / D_{\text {in }}=5$. 


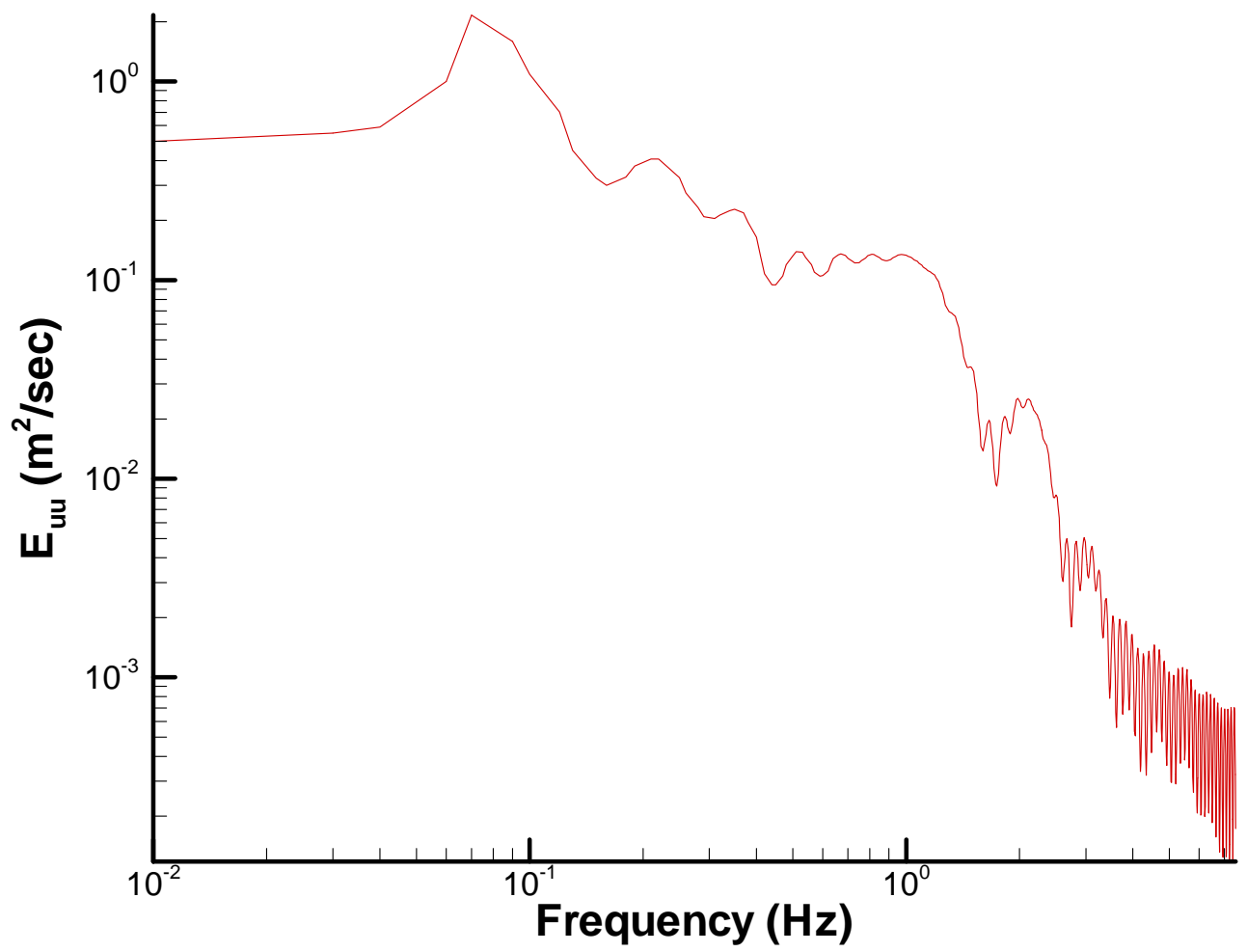

Figure 5.12 - Energy spectrum for $\mathrm{Ri}=0.08$ using $100 \times 213 \times 213$ grid size at $\mathrm{y}=0.5$; $\mathrm{dt}=\mathbf{0 . 0 0 1}$ seconds.

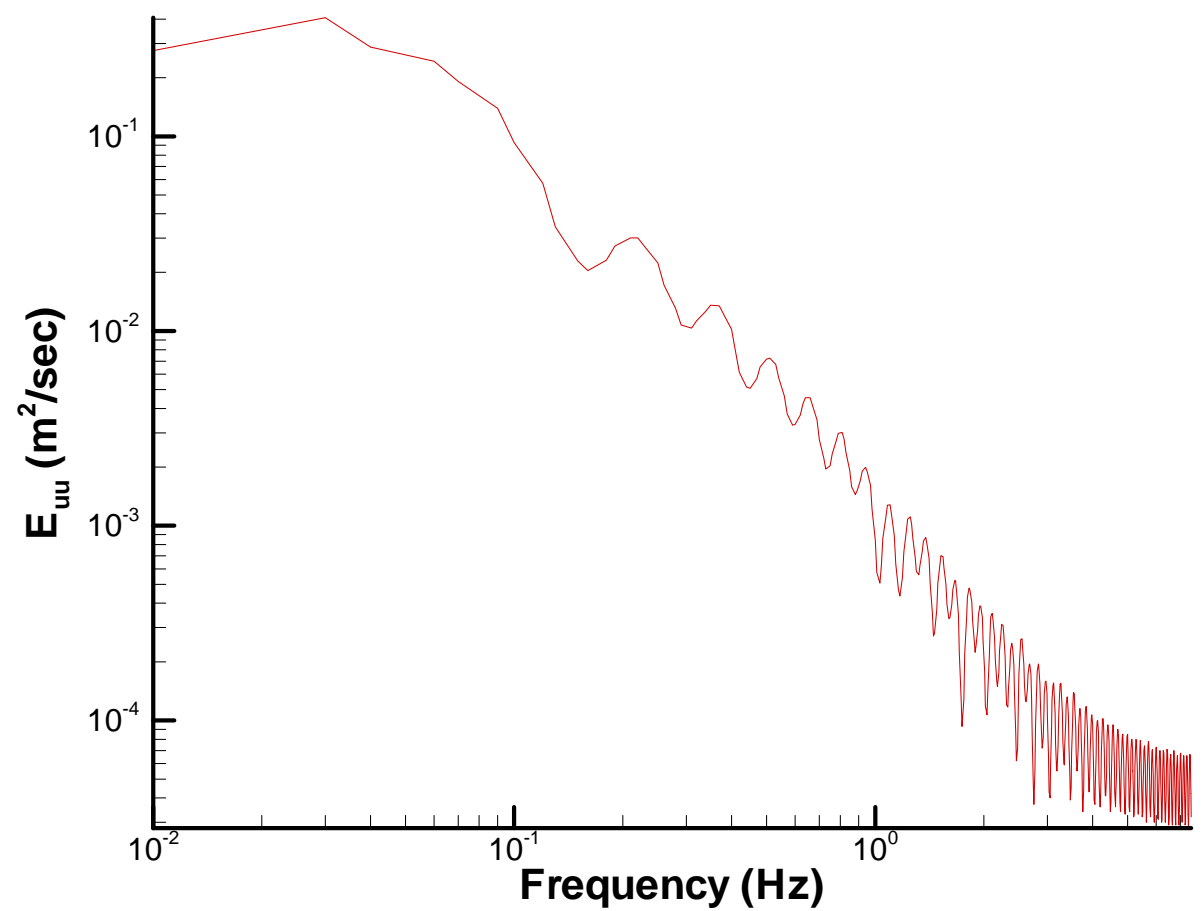

Figure 5.13 - Energy spectrum for $\mathrm{Ri}=0.08$ using $100 \times 213 \times 213$ grid size at $\mathrm{y}=$ $0.525 ; \mathrm{dt}=0.001$ seconds. 


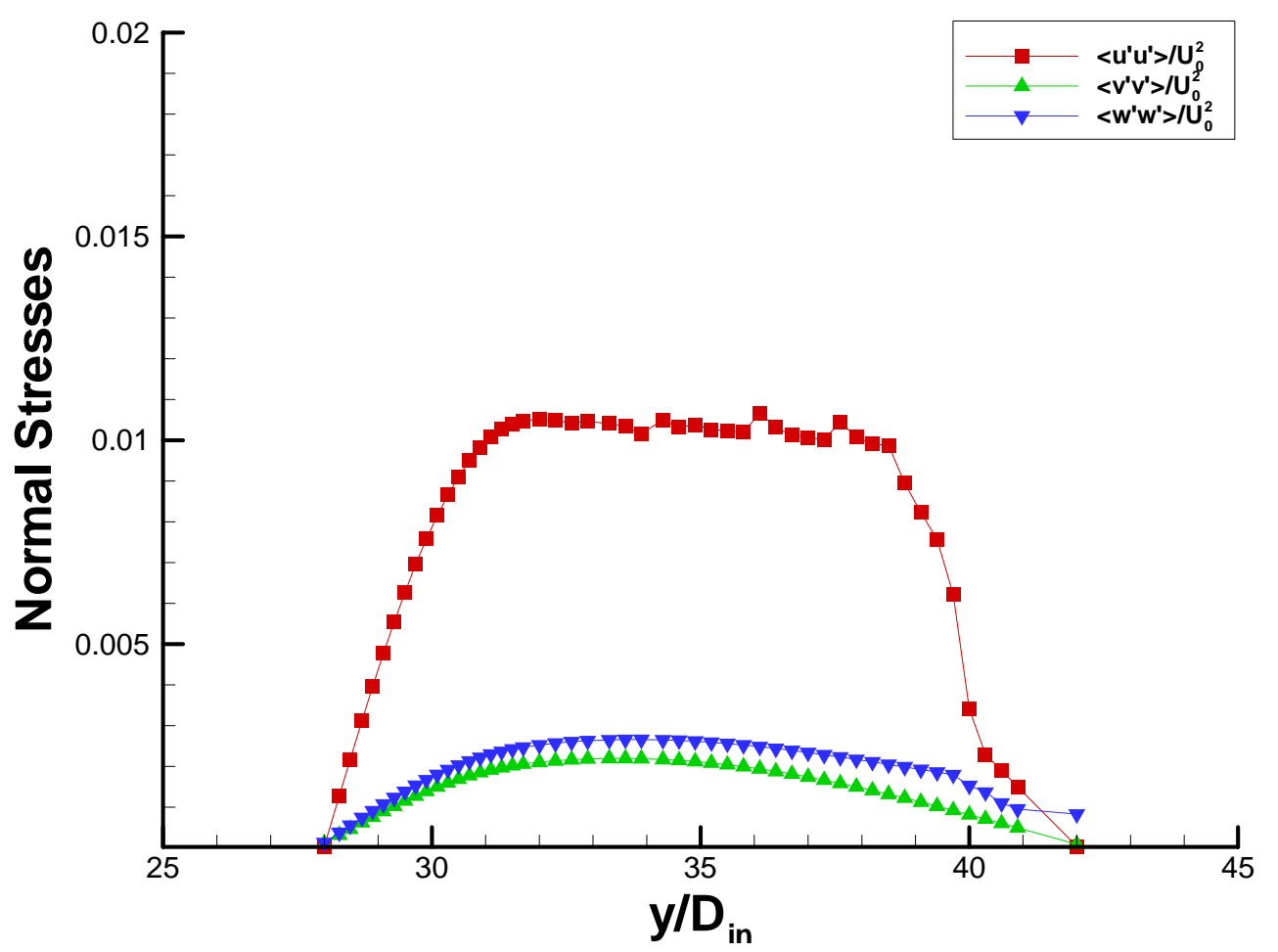

Figure $5.14-$ Normal stresses for $\mathrm{Ri}=\mathbf{0 . 0 8}$ using $100 \times 213 \times 213$ grid size at $\mathrm{x}=\mathbf{0 . 1 7 5}$ across the jet; $\mathrm{dt}=\mathbf{0 . 0 0 1}$ seconds. Averaging time $=\mathbf{1 8 . 0}$ seconds.

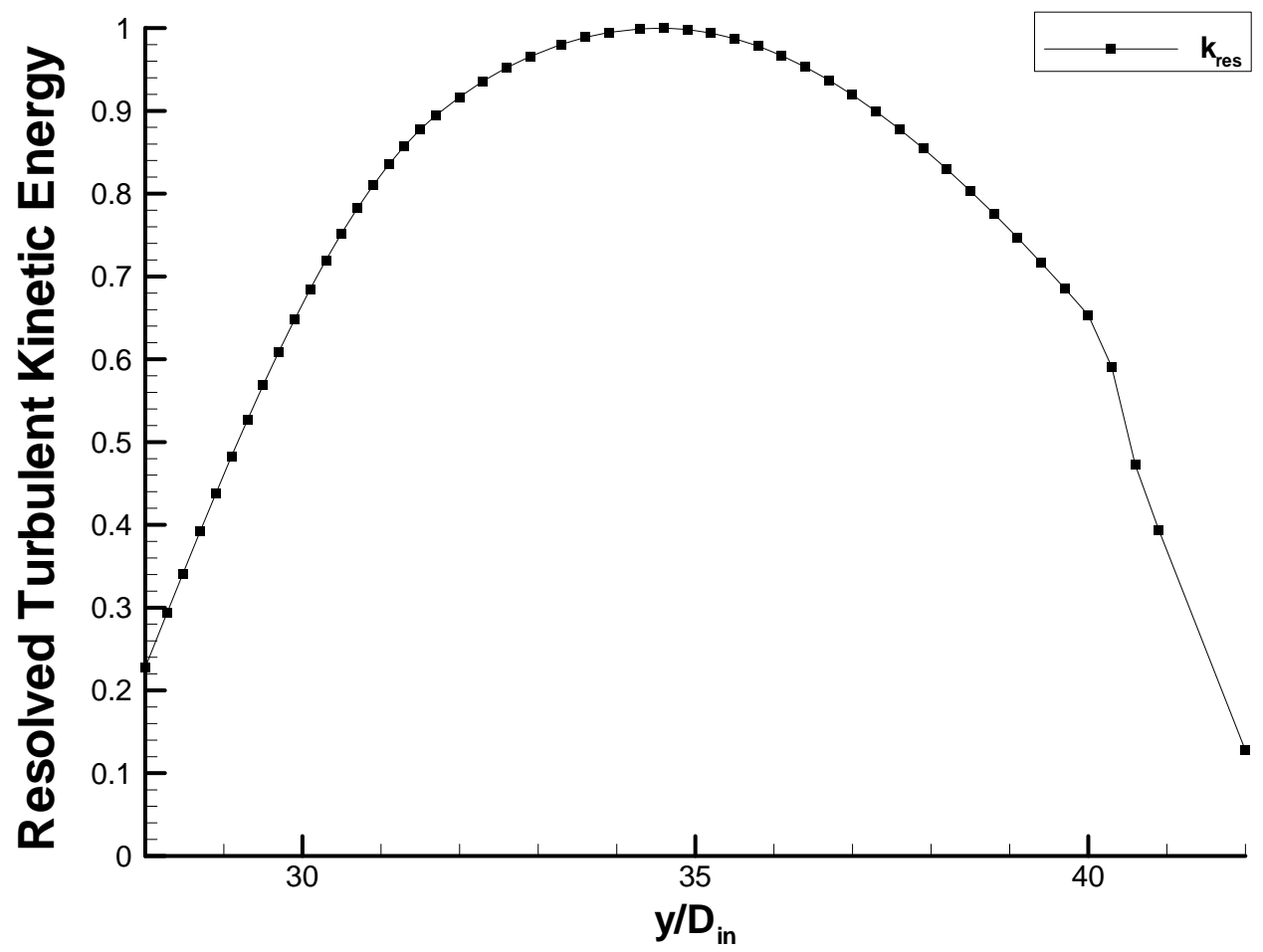

Figure 5.15 - Normalized resolved turbulent kinetic energy for $\mathrm{Ri}=\mathbf{0 . 0 8}$ using $100 \times 213 \times 213$ grid size at $x=0.175$ across the jet; $d t=0.001$ seconds. Total averaging time $=18.0$ seconds. 


\subsection{Vertical Buoyant Jet}

\subsection{Introduction}

Buoyant jets and plumes are of great interest due to their effect on mixing and entrainment. Plumes "arise" when buoyancy is supplied steadily and the buoyant region is continuous between the source and the level of interest (Turner, 1969). The main difference between a jet and plume is that a jet is momentum-driven, whereas a plume is buoyancy-dominated. A forced plume is a kind of flow between a pure jet and a pure plume and is driven by both buoyancy and momentum. This chapter, as with the previous ones, serves the purpose of validating the variable density implementation. Unlike the previous section, which has a negatively forced buoyant jet, this section is more applicable to the overall goal of this study, to investigate the behavior of a curved buoyant jet within an enclosure.

Generally, for the rest of this chapter, the term plume will mean a forced turbulent plume. In most plume experiments without a chemical reaction, fluid either of higher temperature or of lower density is injected into the ambient, resulting in density inhomogeneity, which can produce different types of phenomena. Forced jets and plumes are similar in many respects, such as transition from laminar to a turbulent state in the near-field, self-similarity in the far-field, spreading rate, etc (Zhou et. al., 2000). This chapter will examine some of these characteristics as simulated using DREAM ${ }^{\circledR}$ and compare the DREAM ${ }^{\circledR}$ simulations to the available experimental data for two different plumes, with and without an axisymmetric forcing function. In both studies, the jet is a confined, compounded jet.

\subsection{Description of Experiments}

\subsubsection{Vertical Buoyant Jet}

The behavior of a vertical jet within an enclosure is of considerable importance in the design of jet pumps, refueling of compensated fuel ballast tanks, the study of combustion, etc. The expansion of the jet within the tank is difficult to predict using CFD if improper grid resolution is used, as the interface behavior may be difficult to capture. Figure 5.2.1 shows a schematic representation of the enclosure with a jet of diameter $\mathrm{d}$, issuing axially with a uniform velocity into a duct of diameter $\mathrm{D}$. In these 
types of flows, the issuing jet is known as the primary stream. In the experimental case performed by Razinsky and Brighton (1971) and Chen and Rodi (1980), the surrounding fluid is moving in the same direction as the primary stream and is called the secondary stream. The boundary layer on the wall is neglected in the experiments and is assumed to be negligible, as the secondary stream effects near the wall are minimal. The investigation of the curved buoyant jet within an enclosure is investigated in Chapter 7; however, this section validates and investigates DREAM $^{\circledR}$ and the performance for buoyant flows. In the vertical buoyant jet flows, the buoyancy acts to accelerate the jet, which quickly turns the flow from laminar to turbulent.

Pure plumes are difficult to experimentally investigate due to the relatively small flow velocities within the plume, which is why a forced plume is investigated by most researchers. As with all turbulent flows, they generally start out as laminar flows, then transition to turbulence, which is why Anwar (1968) used a densimetric Froude number that was large enough to ensure that the transition to turbulence occurred near the inlet. This also made sure that the flow was fully turbulent in the range covered by the measurements. Anwar (1968) found that the jet spread linearly and the center-line velocity and density varied with $\mathrm{x}$ as predicted by similarity analysis. The measured profiles within the data acquisition area are closely Gaussian. The experiments used by Anwar (1968) ensured that the flow was turbulent after $x / D=5$, where $x$ is the axial distance and $\mathrm{D}$ is the inlet diameter of the jet. The inlet mass flux of the primary water jet equaled $20.37 \mathrm{~kg} / \mathrm{sec}^{*} \mathrm{~m}$, which yields an inlet Reynolds number of 1892 for the primary stream. The inlet densimetric Froude number was 2.8. The secondary velocity was 50 times less than that of the primary stream, which in the experiments was the ambient salt water, with a density of $1032 \mathrm{~kg} / \mathrm{m}^{3}$. The distance between the walls in the spanwise and transverse direction equaled $0.3 \mathrm{~m}$. These parameters are matched in the DREAM $^{\circledR}$ simulations, with the results and comparisons presented in section 6.4. The geometry used in the experiments is shown in Figure 6.1. 


\subsubsection{Vertical Buoyant Jet with Axisymmetric Forcing}

The turbulent jet has been a subject of investigation for the last century. Similarity within the jet has long been known, even for stratified environments, although measurements of the turbulence properties is still a fundamental calibration tool. For this reason, vertical forced plumes are used as a verification of $\mathrm{DREAM}^{\circledR}$ in this study.

George and Tamanini (1977) performed experiments to measure turbulence in an axisymmetric, forced buoyant plume. Their experiments were designed to ensure that the forced plume achieves its turbulent state within two inlet jet diameters. Other investigators; however, have shown that the potential core may be extended to 5-8 inlet jet diameters depending on the mode of fluctuation imposed on the jet at the inlet, as there are several ways to achieve an earlier spatial development of turbulence. Higher forcing of perturbations can also reduce the length of the laminar zone. For this reason, George and Tamanini (1977) adopted a higher level of forcing in the experiments. The inlet diameter used in the experiments was $0.018 \mathrm{~m}$, with the internal Froude number equaling 1.54. This value corresponds to a densimetric Froude number of 1.93, and a Reynolds number of 1273 . The average inlet velocity equaled $0.98 \mathrm{~m} / \mathrm{sec}$.

For these experiments, it was difficult to measure the velocity profile at the inlet, which is needed for any CFD simulation. Thus, to accurately determine this parameter, Michalke (1984) determined that the primary inlet velocity profile for the jet was that of a top-hat velocity profile, given by

$$
U_{o}(y)=0.5 * U_{o}[1-\tanh [12.5(2 y / D-D / 2 y)]]
$$

where $\mathrm{U}_{\mathrm{o}}=0.98 \mathrm{~m} / \mathrm{sec}, \mathrm{D}$ is the inlet diameter, and $\mathrm{y}$ is the vertical location within the jet, all of which are from the experiments by George and Tamanini (1977). Michalke (1984) states that the length of the potential core strongly depends on the disturbances added at the inlet. To excite the flow, a fluctuating axial velocity at the inflow takes the following form (Michalke (1984))

$$
u^{\prime}=A U_{o}(y) \sin (0.3 \pi t)
$$

where $\mathrm{A}$ is the amplitude of the forcing, which equaled 0.2 , and $\mathrm{t}$ is the time. The other velocity components are forced with smaller amplitudes of the mean axial velocity 
equaling $0.01 \mathrm{U}_{\mathrm{o}}$, which also corresponds to the secondary inlet velocity. The geometry of the experiments used by George and Tamanini (1977) are shown in Figure 6.2. These parameters are matched in the DREAM ${ }^{\circledR}$ simulations.

\subsection{Computational Details}

Simulations have been carried out for the two different vertical jet setups, as presented in Section 6.2. In all cases, the jet axis is aligned with the gravity vector and is subject to a positive buoyancy force. The inlet density equaled $920 \mathrm{~kg} / \mathrm{m}^{3}$ with the ambient density being $1000 \mathrm{~kg} / \mathrm{m}^{3}$, respectively. The dimensionless parameters used in the DREAM ${ }^{\circledR}$ simulations are set to be identical to those of the experiments. The Froude and Reynolds numbers were made similar through inlet jet diameter modification, which is taken to be $0.02 \mathrm{~m}$ for comparison to the Anwar (1969) experiments, and $0.018 \mathrm{~m}$ for comparison to the George and Tamanini (1977) experiments. All boundary conditions except at the inlet and outlet plumes are taken to be walls, which correspond to the confined vertical buoyant jet experiments. The total non-dimensional axial length equaled 24 inlet diameters for comparison to the Anwar (1969) experiments, and 32 inlet diameters for comparison to the George and Tamanini (1977) experiments.

For comparison to each experiment, two different non-uniform grids were used, being 75 x 57 x 57 and 150 x 107 x 107. These are shown in Figures 6.3 and 6.4 for the Anwar (1968) simulations; the geometry is similar to the George and Tamanini (1977) experiments. A uniform grid is used in the axial direction for all simulations, with the non-uniformity being imposed in the spanwise and transverse directions. The first grid size for the fine grid, away from $y=0$ and $z=0$, equaled 0.000369 meters for the Anwar (1969) simulations and 0.00031 meters for the George and Tamanini (1977) simulations. The coarse grid location equaled 0.000751 meters and 0.000669 meters for the Anwar (1969) and George and Tamanini (1977) simulations, respectively. In both cases, a uniform expansion ratio equaling two percent was applied. This yielded approximately 35 and 10 grids within the inlet jet region for the fine and coarse grid simulations, respectively. Since the current interpolation scheme does not fully take into account the nonuniformity in the mesh, this may have caused some additional errors in accuracy in the simulation results. The time-steps used in simulating the Anwar (1968) and George 
and Tamanini (1977) experiments equaled 0.00025 seconds. These time-steps were utilized to keep the Courant number less than approximately 0.2. The total real simulation time for the coarse and fine grids, ensuring four flow through times, equaled approximately sixteen hours and four days run on quad processor XEON workstations.

\subsection{Results and Conclusions}

In this section, the aim is to answer a variety of questions about the ability of DREAM $^{\circledR}$ to accurately simulate buoyant flow events, and particularly buoyant jets. As mentioned in Chapter 3, DREAM ${ }^{\circledR}$ uses a variation of the projection method, accompanied with a Crank-Nicholson discretization scheme. A comparison between the coarse and fine grid simulations are examined in this section, which will clearly show not only a time-step dependence, but also a grid size dependence of the results. This section clearly shows that "sufficient" grid resolution, accompanied with "adequate" time-steps, is crucial to success of the implicit turbulence methods. All simulations presented in this section have been run for approximately four flow-through times, which allows for an "adequate" statistical analysis of the simulation data. Also, it is important to note that in the statistical analysis, a combined spatial and time averaging approach is used in the analysis of the turbulence quantities.

\section{Uniform Inlet Velocity}

Figure 6.5 and 6.6 show the velocity magnitude contours for the coarse and fine grid simulations. Clearly, the results in Figure 6.5 exhibit too much dissipation, and not capable of resolving the small scale eddies. The jet boundaries are not engulfing the surrounding fluid to enable a suitable spreading rate of the jet. This phenomenon becomes clearer in comparison of Figures 6.7 and 6.8, which show the instantaneous velocity vectors for the coarse and fine grid simulations. Figure 6.7 appears laminar, with no vectors showing any type of mixing taking place. This is clearly not the case, in Figure 6.8. The velocity vectors appear more random and engulfing. Comparison of Figures 6.7 and 6.8 alone show the necessity of adequately resolving the jet. Again, the time-step was determined to ensure that the Courant number was under 0.2. For a flow 
field to be considered turbulent, small scale fluctuations of the mean must be evident. Figures 6.9 and 6.10 show the instantaneous streamwise velocity at a point within the jet, directly in the center of the computational domain $\left(\mathrm{x} / \mathrm{D}_{\text {in }}=12.5 ; \mathrm{y} / \mathrm{D}_{\text {in }}=0.0 ; \mathrm{z} / \mathrm{D}_{\text {in }}=0.0\right)$, versus time. The data shown in Figure 6.9 are the DREAM ${ }^{\circledR}$ simulations for the coarse grid simulations, and although they indicate some small fluctuations toward the end (which might have grown if the run was continued much longer) of the simulations, these results under-predict the amount of turbulence within the flow, which is clearly visible in Figure 6.10.

A clear indication of the turbulence present within the flow is the vorticity. Again, the coarse grid simulation, Figure 6.11, of the z-component vorticity contour is under-resolved and it is seen that runs with this grid are incapable of capturing the flow field physics. Figure 6.12 shows the finer grid simulations, and appears to be the minimum resolution capable of capturing the different sized eddies within the flow. Figures 6.13 and 6.14 show the vorticity magnitude contours, which yield a similar conclusion concerning the grid resolution.

The density contours (Figures 6.15 and 6.16) show a similar phenomenon. Figure 6.15 shows no mixing or entrainment at the boundaries, which is necessary to adequately predict the jet spreading rate. The reason is that the grid is too coarse at the boundaries, and consequently under-predicts the shear at those locations. This causes the flow field to appear laminar. As no entrainment function is used in the simulations, the mixing and spreading rate of the jet is dependent on the ability to appropriately capture the shear stress at the interface. When an appropriate grid resolution is used, DREAM ${ }^{\circledR}$ does appear to have the ability to accurately resolve the interface and appropriately predict the interface physics (Figure 6.16). Further study of this phenomena is indeed needed for quantification.

Figure 6.17 shows a comparison between the DREAM ${ }^{\circledR}$ simulations and Anwar (1964) experimental data for the normalized half-jet velocity at $\mathrm{x} / \mathrm{D}_{\text {in }}=15$ and 20 for the finer grid simulations. Since, the coarse grid simulations are too dissipative, they are not included in the analysis of the turbulence quantities. The tendency for a buoyant jet to become self-similar some distance downstream means that the turbulent flow depends only on the initial momentum. Similarity is visible in both the experiments and 
DREAM $^{\circledR}$ simulations, which lends weight to the validity of the present simulations with the fine grid. A similar trend is seen in analysis of the turbulence quantities as well. Figures $6.18-6.20$ show the $\mathrm{u}, \mathrm{v}$, and w r.m.s velocity components as compared to the experimental data. Not only do the DREAM ${ }^{\circledR}$ simulations compare reasonably well to the experiments by Anwar (1969), but the simulation also shows the principal of similarity, as expected. The data has been time averaged over two flow-through times, as well as spatially averaged over eight nearest neighbor adjacent cells on the centerlines. A comparison of the resolved turbulent kinetic energy from the DREAM ${ }^{\circledR}$ simulations to the experimental data is shown in Figure 6.21, which shows excellent agreement. Calculated normalized total energy spectra between the coarse and fine grid simulations at $\mathrm{x} / \mathrm{D}_{\mathrm{in}}=15$ and 20 , again clearly shows that the coarse grid doesn't adequately predict the trend of the Kolmogorov spectra, and further supports the omission of presentation within this section (Figures $6.22 \mathrm{a}$ and b). It is important to note that all statistical analysis uses the techniques as explained in Section A.5, including the calculation of the normalized total energy spectra, and will not be explained here. The reader is referred to Section A.5 for further details.

\section{Axisymmetric Forcing at the Inlet}

The previous section presented results showing the ability of DREAM $^{\circledR}$ using LES ITM type methods in the simulation of constant inlet velocity, buoyant flow situations. This section presents the results from using the forcing function, Eq. 6.2.1 and 6.2.2, as presented in Section 6.2. As the geometries between the two different experiments were similar, identical grid sizes were used, with a slightly increased grid resolution in these simulations. Figure 6.23 and 6.24 shows the coarse and fine grid resolutions used in the DREAM ${ }^{\circledR}$ simulations. Similar conclusions to those reached in the previous section may be drawn with regard to using a coarse or fine grid in the simulations, with the coarse grid simulations again being too dissipative. This can be seen for the velocity magnitudes (Figures 6.25 and 6.26), velocity vectors (6.27 and $6.28)$, z-component vorticity (6.29 and 6.30), the vorticity magnitude (6.31 and 6.32) and the density contours (6.33 and 6.34). 
As in the previous sub-section, if self-similarity is achieved, same shapes should be maintained at different downstream locations for the same mean velocity. Two axial positions ( $\mathrm{x} / \mathrm{Din}=8$ and 14) were chosen to examine the jet behavior. Figure 6.35 shows the normalized velocity across the jet half width. These results compare well with the experimental data by George and Tamanini (1977) and display the expected similarity properties. The normalized r.m.s of the $\mathrm{x}, \mathrm{y}$, and $\mathrm{z}$ velocity components are shown in Figures 6.36, 6.37, and 6.38. It is observed that the turbulence intensities in the $\mathrm{y}$ and $\mathrm{z}$ direction are smaller in the axial direction, which is expected and evident from numerous jet experiments. The peak is off-axis in the profile of axial velocity fluctuations and some shift in the experiments is also visible, which may be due to insufficient sampling. The radial profiles of the turbulent density fluctuations are shown in Figure 6.39, where it can be seen that the centerline value of the scalar intensity is approximately 0.40 . This is substantially higher than the $0.20-0.25$ velocity fluctuations in jets and is due to buoyancy intensifying the turbulence field. This is clear in vertical buoyant jet phenomena, but is less evident in the curved buoyant jet simulations presented in Chapter 7.0. Self similarity is not as evident in the r.m.s density fluctuations, which is more difficult to achieve. A comparison of the measured turbulent kinetic energy to the resolved turbulent kinetic energy is shown in figure 6.40 at the two axial locations. Again, the results correlate well with the observed experimental data. The normalized total energy spectrum is presented in Figure 6.41, with the fine grid simulations closely obeying the Kolmogorov spectra. However, it is important to note that although the slope of the Kolmogorov spectra is roughly observed, there appears to be no strong energy containing peak. This further indicates the need for increased resolution in the simulations.

Thus, in conclusion, adequate grid resolution and time step size are required to produce meaningful results using LES and ITM. Use of a coarse grid yields too much numerical diffusion, as does a larger time step size. Increasing the time step size by a factor of four also yielded too much diffusion in the fine grid simulations. Thus, it is necessary to use "sufficiently" small time step to prevent the projection method from filtering out too much of the lower frequency statistics, which is a characteristic of ITM methods. 


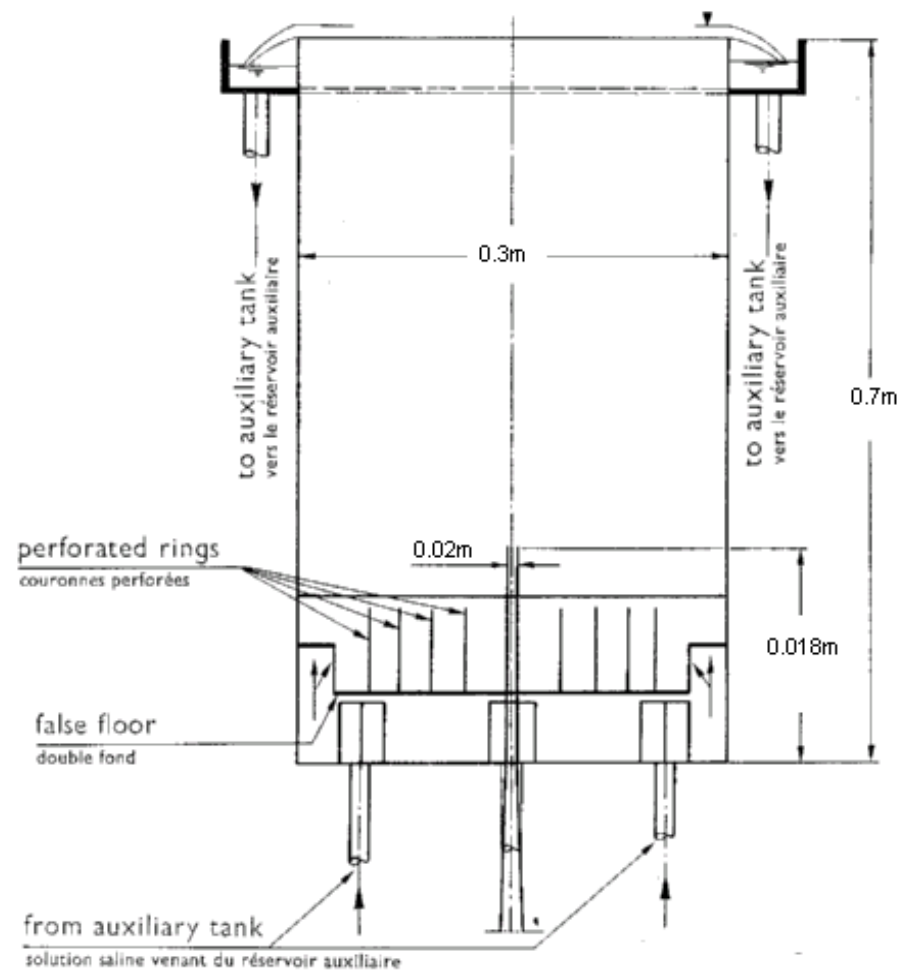

Figure 6.1 - Geometry used in Anwar (1968) experiments

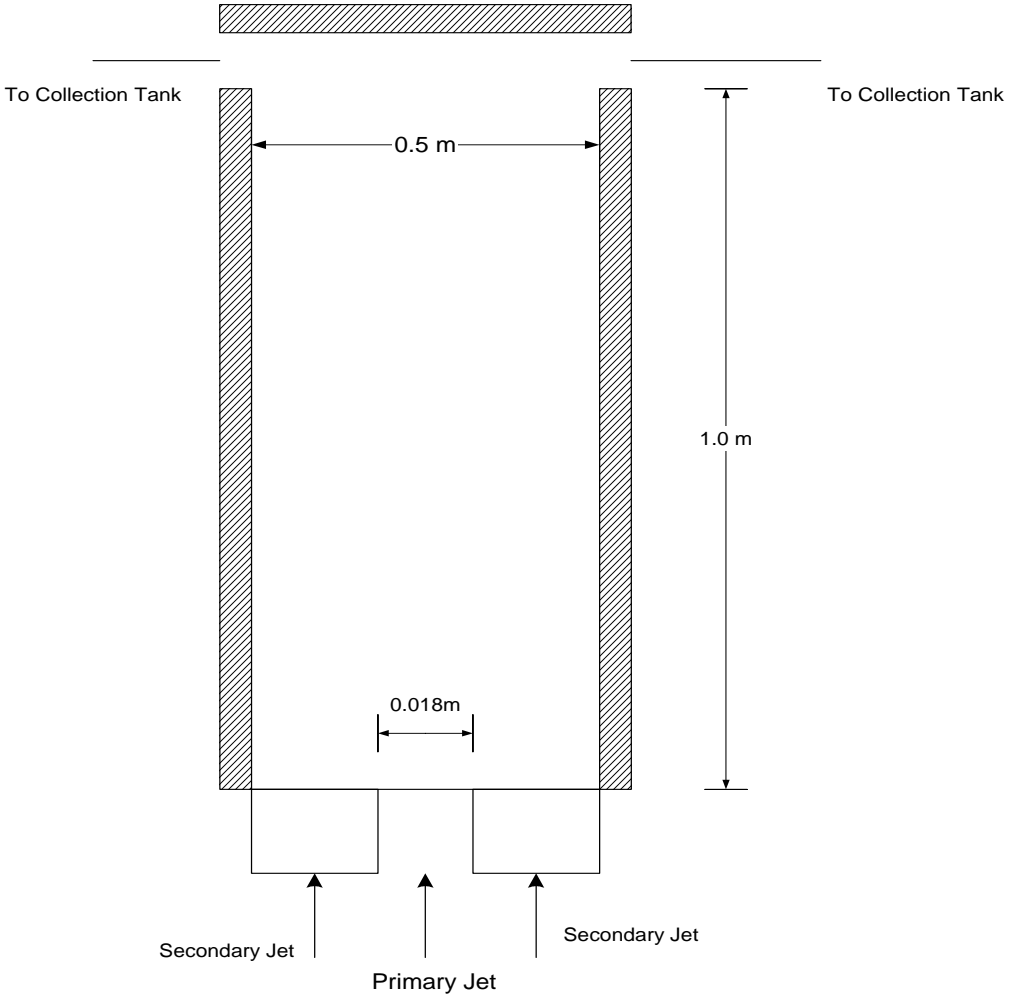

Figure 6.2 - Geometry used in the George and Tamanini (1977) experiments 


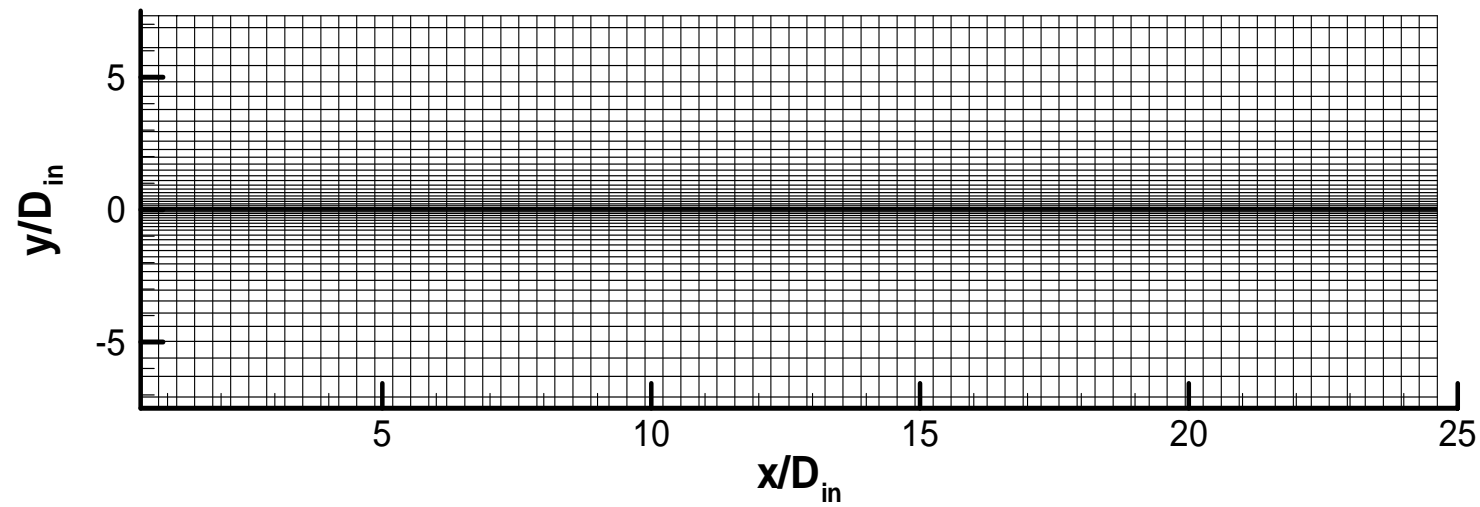

Figure 6.3 - Coarse grid used in the DREAM ${ }^{\circledR}$ simulations for Anwar (1964) experiments

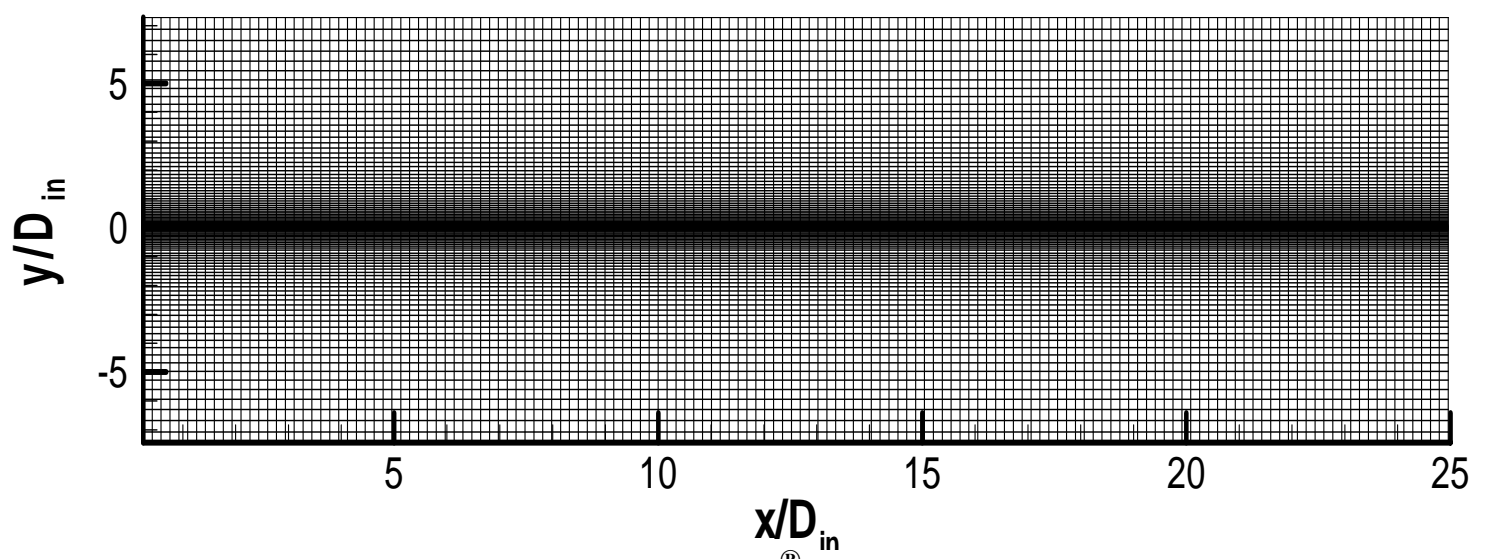

Figure 6.4 - Fine grid used in the DREAM ${ }^{\circledR}$ in ${ }^{\text {im }}$ (alations for comparison to Anwar (1964) experiments 


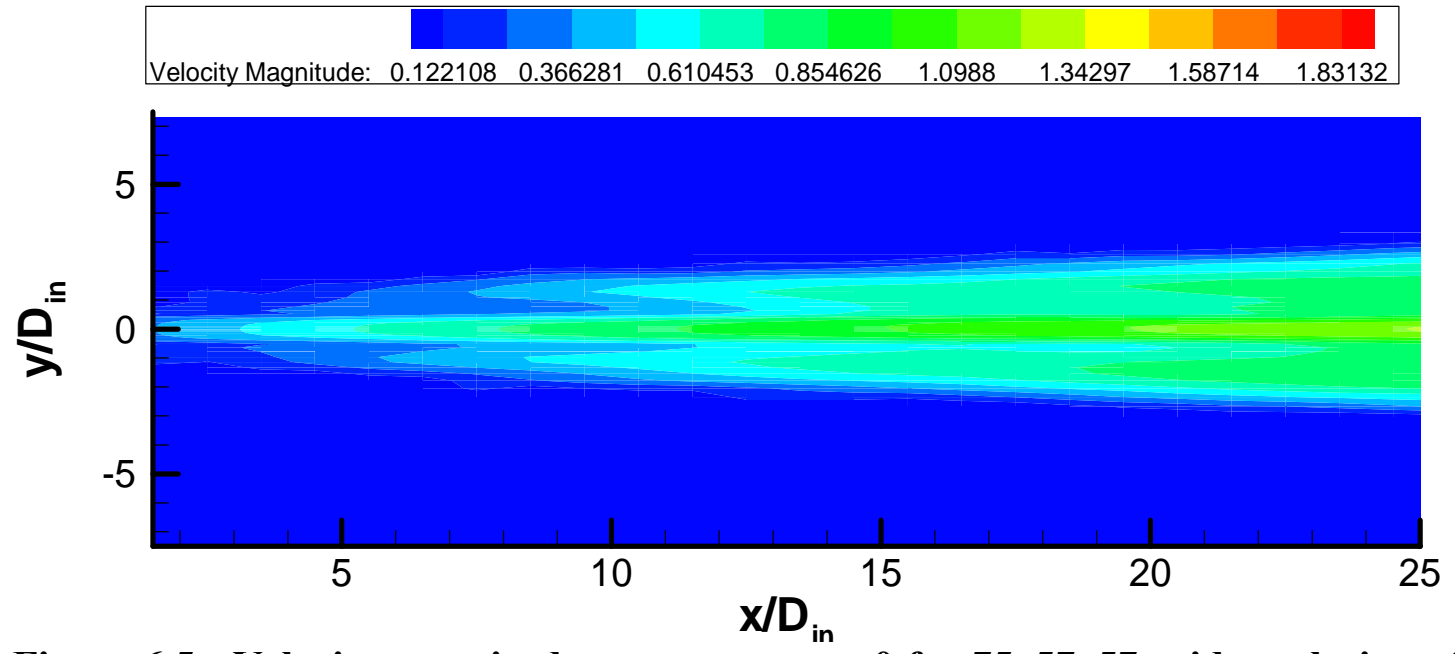

Figure 6.5 - Velocity magnitude contours at $\mathrm{z}=0$ for $75 \times 57 \times 57$ grid resolution after four flow through times; $\mathrm{dt}=\mathbf{0 . 0 0 0 2 5} ; \mathrm{Re}=1892 ; \mathrm{Fr}=2.8$

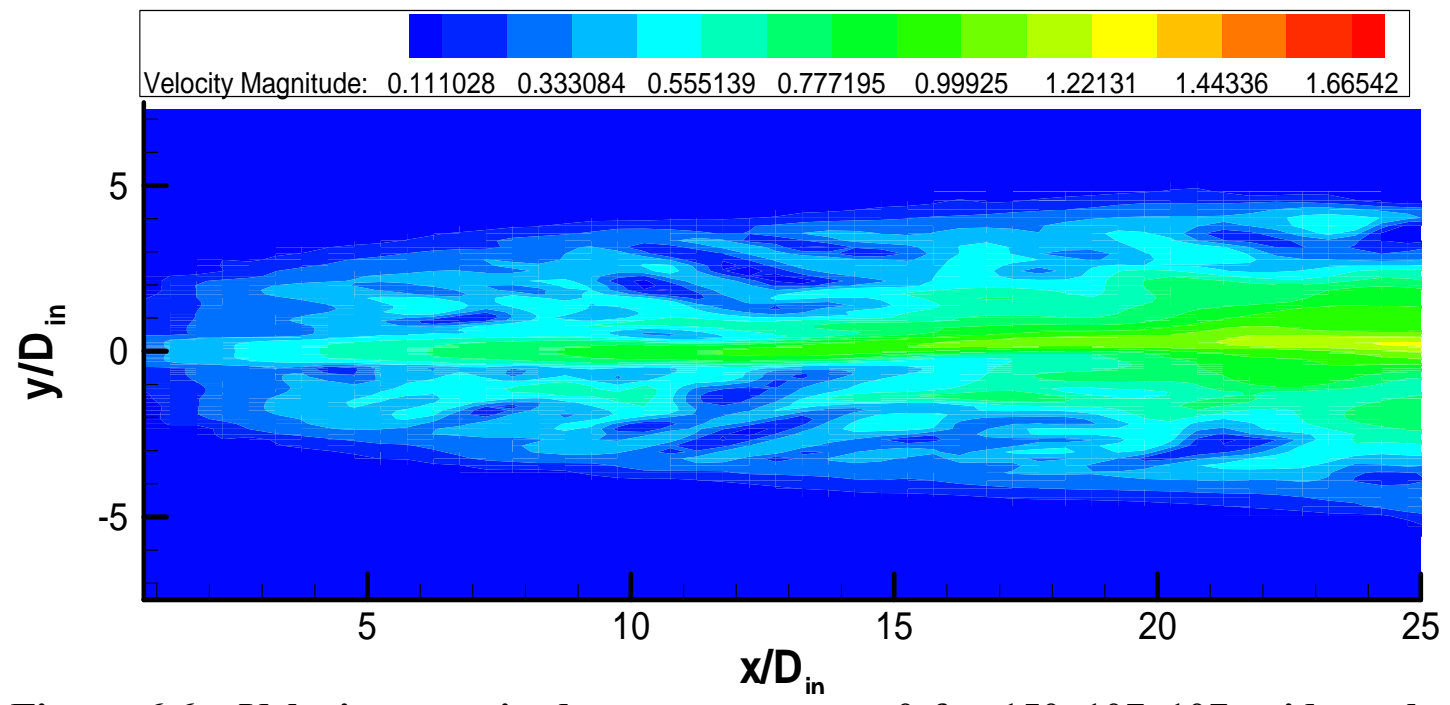

Figure 6.6 - Velocity magnitude contours at $\mathrm{z}=0$ for $150 \times 107 \times 107$ grid resolution after four flow through times; $\mathrm{dt}=\mathbf{0 . 0 0 0 2 5} ; \mathrm{Re}=1892 ; \mathrm{Fr}=2.8$ 


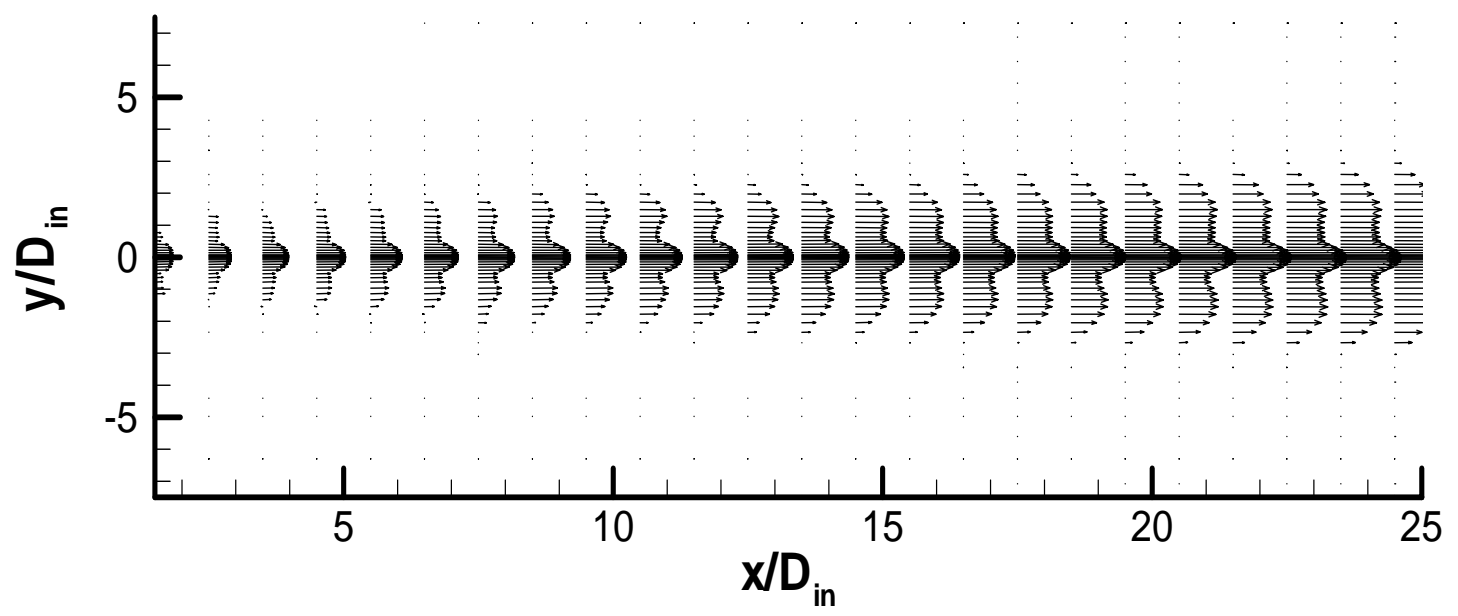

Figure 6.7 - Instantaneous velocity vectors at $\mathrm{z}=0$ for $75 \times 57 \times 57$ grid resolution after four flow through times; $\mathrm{dt}=0.00025 ; \operatorname{Re}=1892 ; \mathrm{Fr}=2.8$

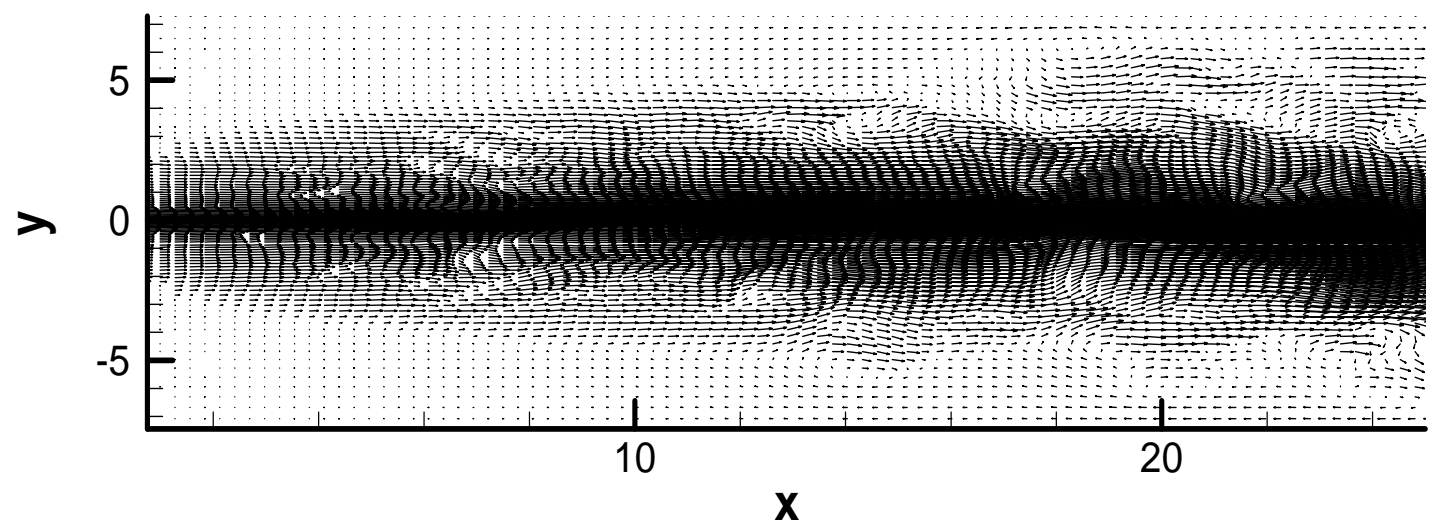

Figure 6.8 - Instantaneous velocity vectors at $z=0$ for $150 \times 107 \times 107$ grid resolution after four flow through times; $\mathrm{dt}=0.00025 ; \mathrm{Re}=1892 ; \mathrm{Fr}=2.8$ 


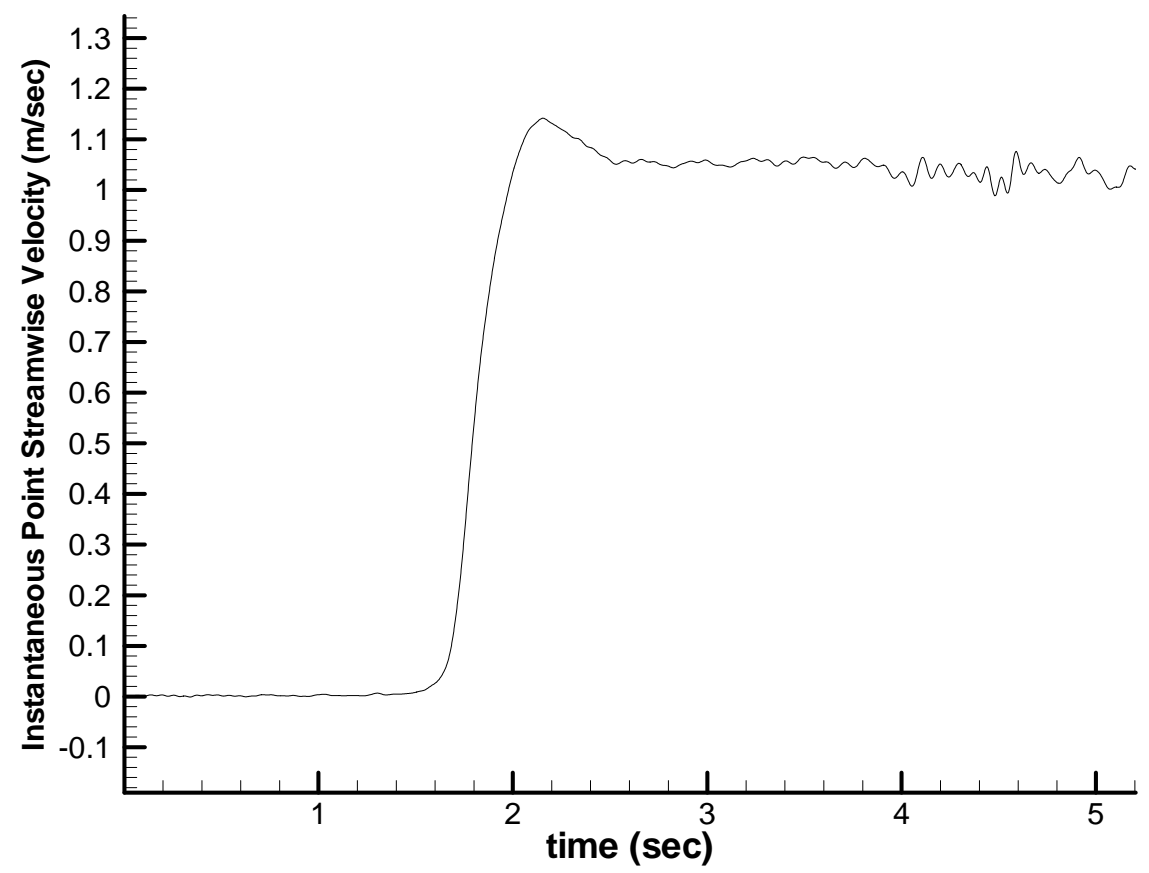

Figure 6.9 - Instantaneous point velocity component versus time for $75 \times 57 \times 57$ grid resolution; $\mathrm{dt}=\mathbf{0 . 0 0 0 2 5} ; \mathrm{Re}=1892 ; \mathrm{Fr}=2.8$

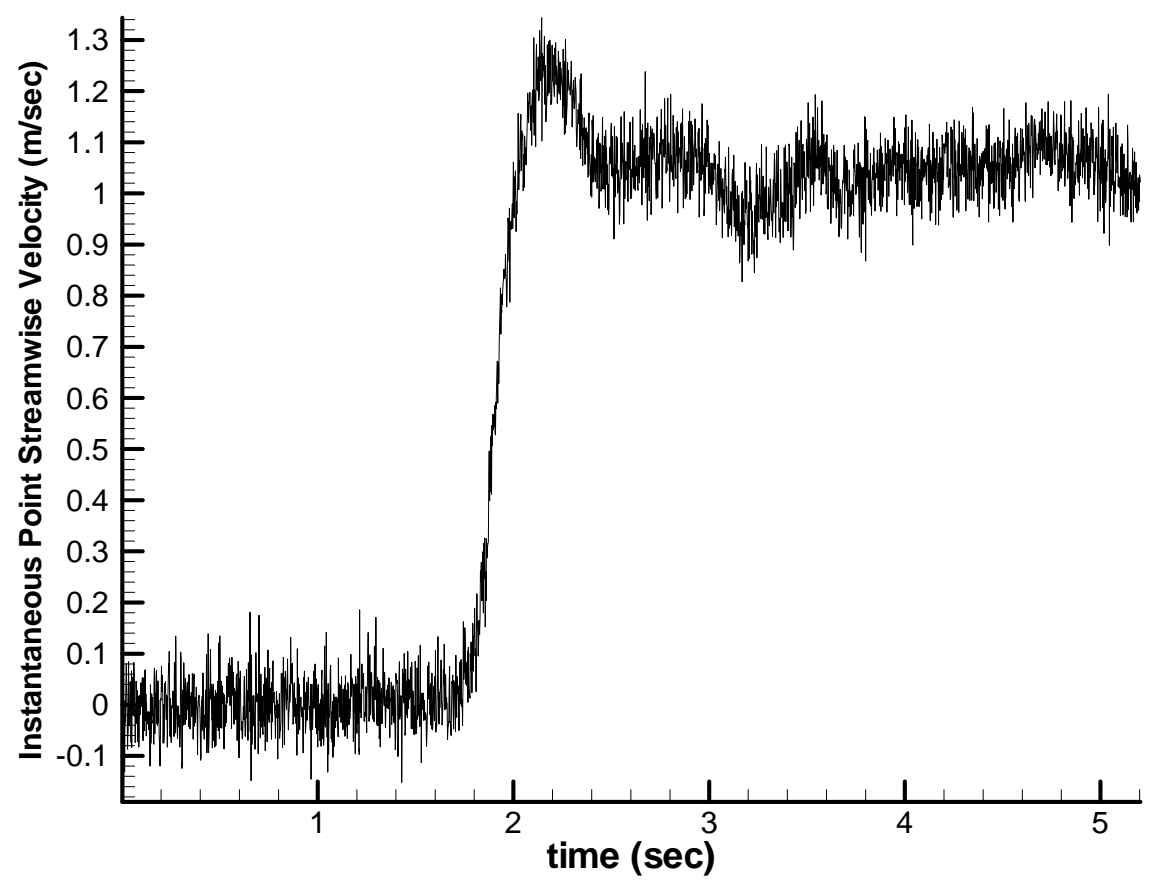

Figure 6.10 - Instantaneous point velocity component versus time for $150 \times 107 \times 107$ grid resolution; $\mathrm{dt}=\mathbf{0 . 0 0 0 2 5} ; \mathrm{Re}=1892 ; \mathrm{Fr}=2.8$ 


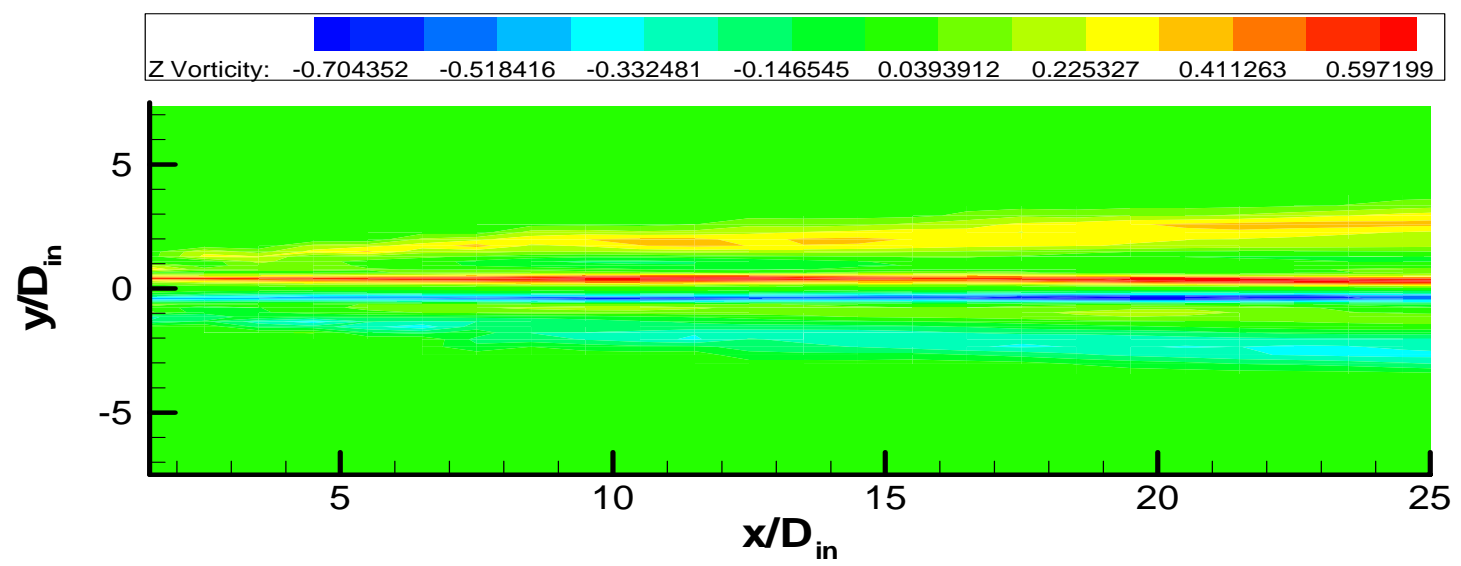

Figure 6.11 - $Z$ component vorticity contours at $z=0$ for $75 \times 57 \times 57$ grid resolution after four flow through times; $\mathrm{dt}=0.00025 ; \operatorname{Re}=1892 ; \mathrm{Fr}=2.8$

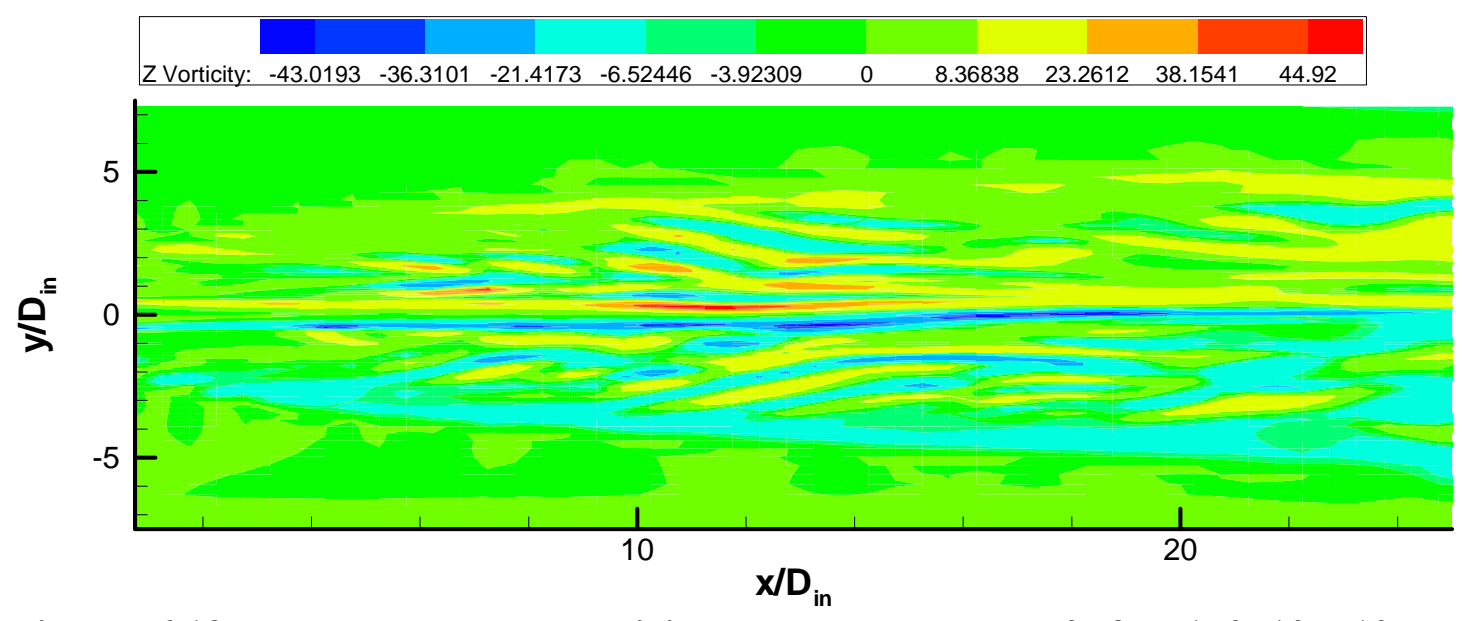

Figure 6.12 - $Z$ component vorticity contours at $z=0$ for $150 \times 107 \times 107$ grid resolution after four flow through times; $\mathrm{dt}=0.00025 ; \operatorname{Re}=1892 ; \mathrm{Fr}=2.8$ 


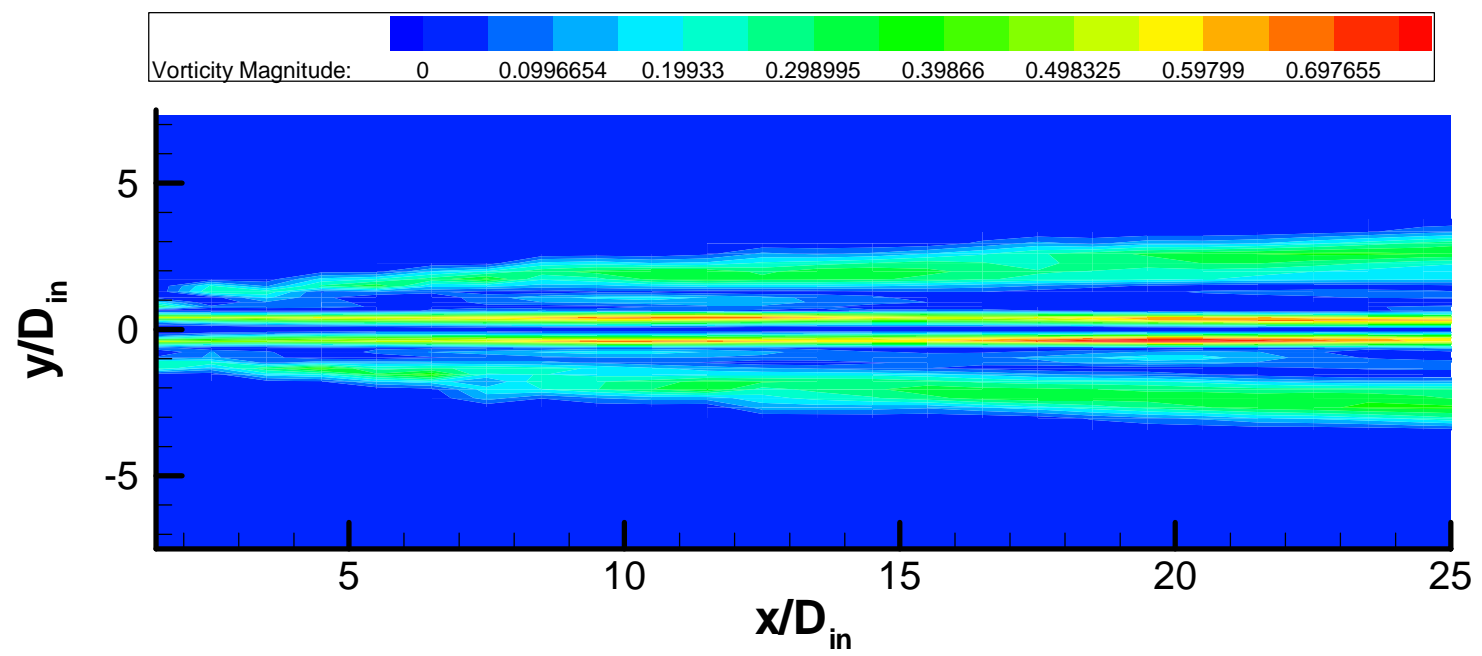

Figure 6.13 - Vorticity magnitude contours at $\mathrm{z}=0$ for $75 \times 57 \times 57$ grid resolution after four flow through times; $\mathrm{dt}=0.00025 ; \mathrm{Re}=1892 ; \mathrm{Fr}=2.8$

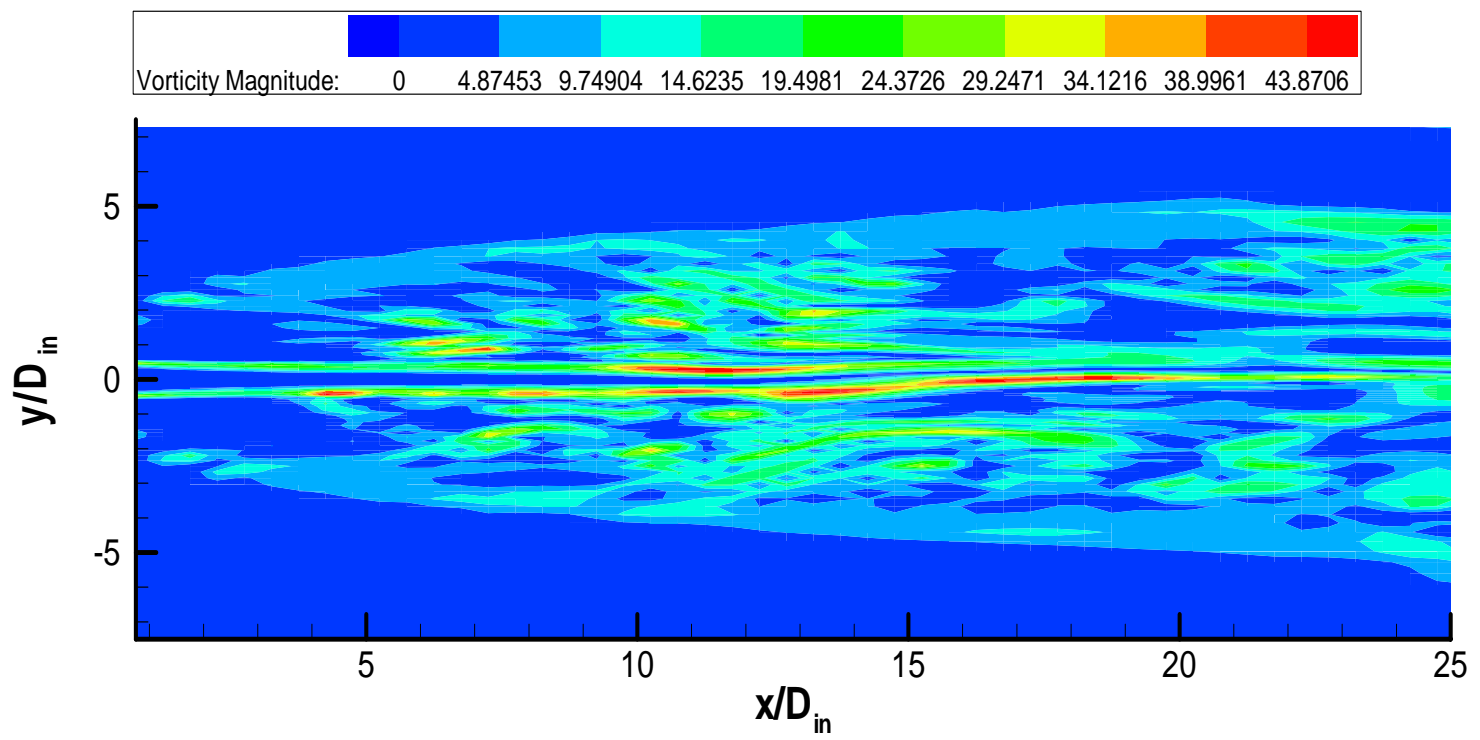

Figure 6.14 - Vorticity magnitude contours at $z=0$ for $150 \times 107 \times 107$ grid resolution after four flow through times; $\mathrm{dt}=0.00025 ; \operatorname{Re}=1892 ; \mathrm{Fr}=2.8$ 


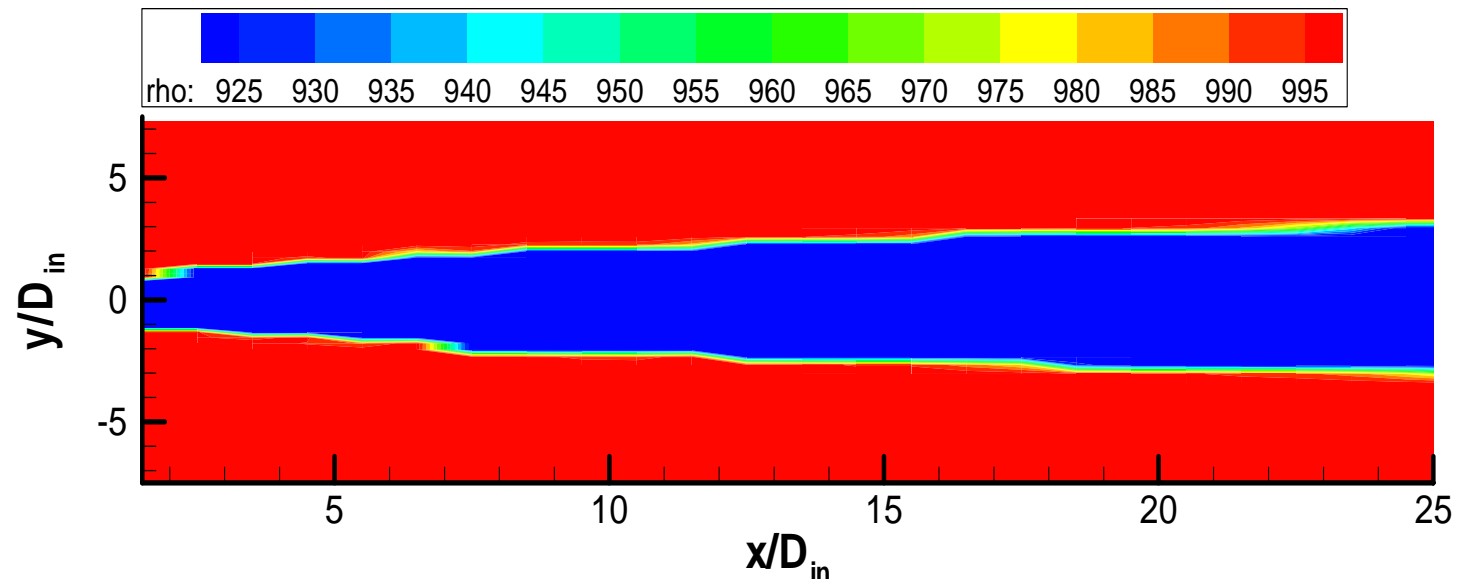

Figure 6.15 - Density contours at $\mathrm{z}=0$ for $75 \times 57 \times 57$ grid resolution after four flow through times; $\mathrm{dt}=0.00025 ; \operatorname{Re}=1892 ; \mathrm{Fr}=2.8$

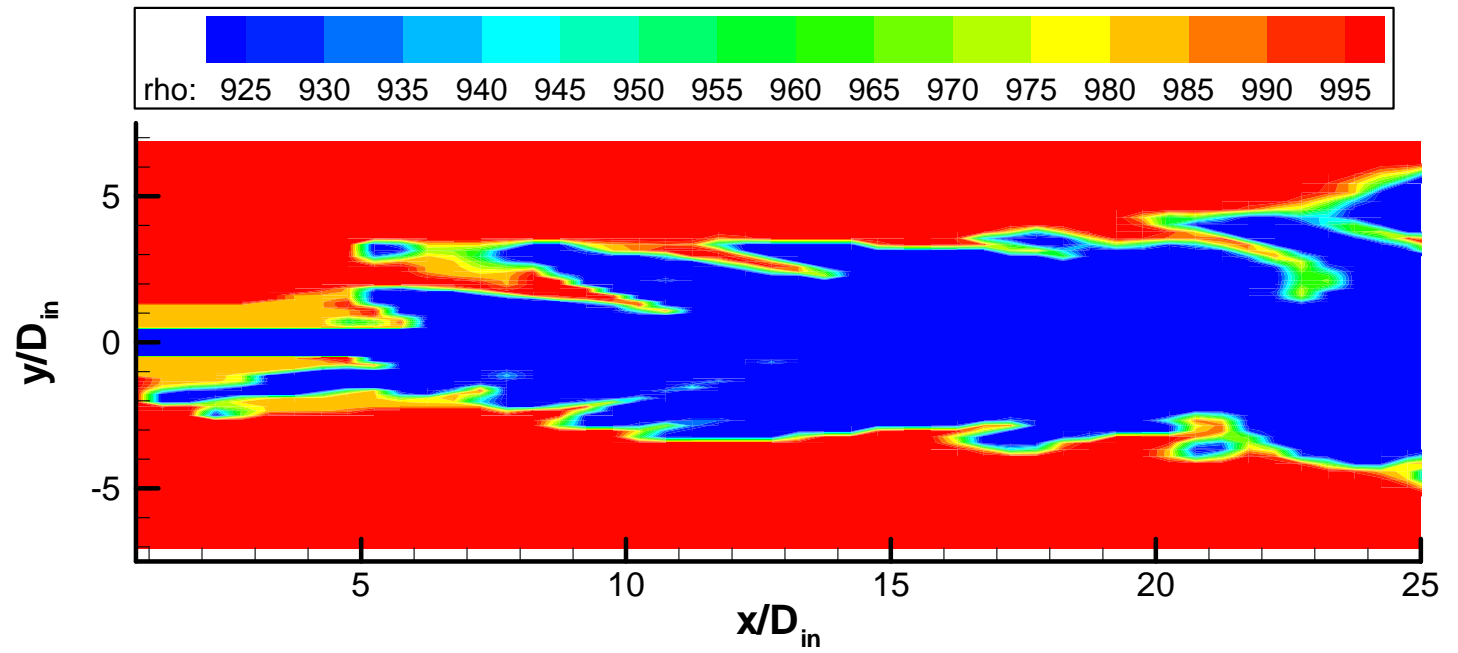

Figure 6.16 - Density contours at $z=0$ for $150 \times 107 \times 107$ grid resolution after four flow through times; $\mathrm{dt}=0.00025 ; \operatorname{Re}=1892 ; \mathrm{Fr}=2.8$ 


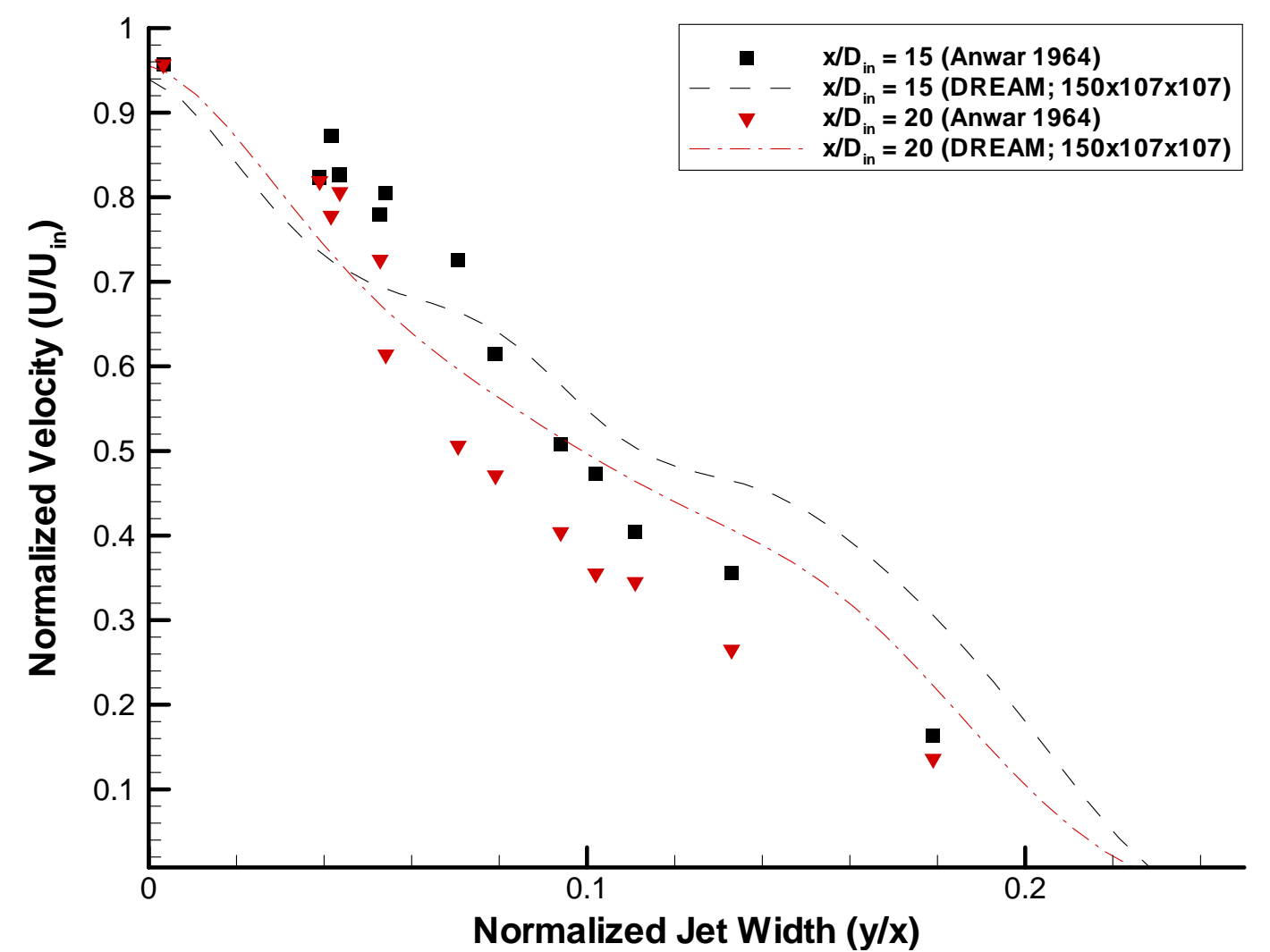

Figure 6.17 - Normalized axial velocity at $x / D_{\text {in }}=15$ and $x / D_{\text {in }}=20$ from DREAM ${ }^{\circledR}$ simulations to experiments; $\mathrm{dt}=0.00025 ; \mathrm{Re}=1892 ; \mathrm{Fr}=2.8$ 


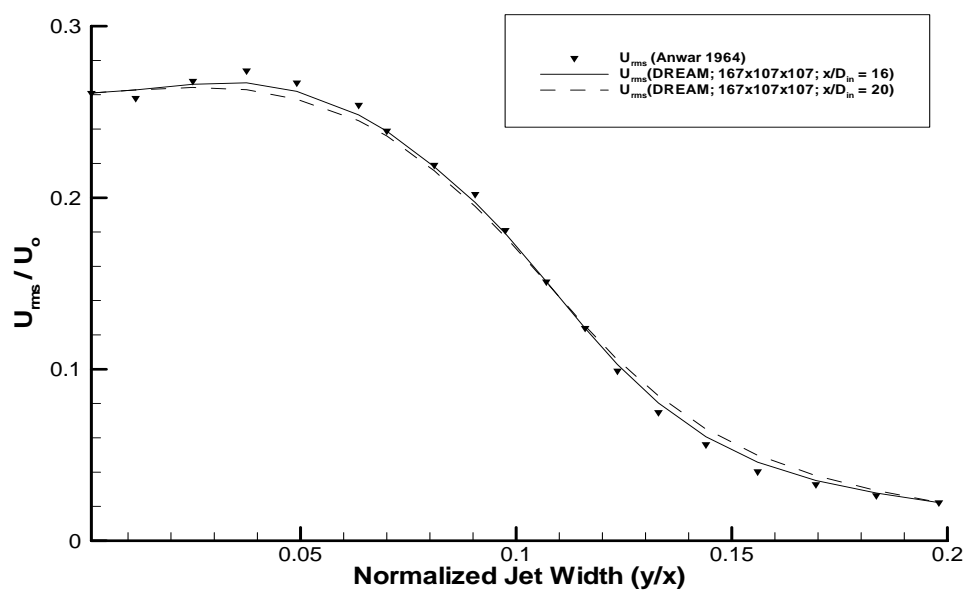

Figure 6.18 - Comparison of $U_{\text {rms }}$ from DREAM ${ }^{\circledR}$ simulations to experiments at $x / D_{\text {in }}=15$ and $x / D_{\text {in }}=20 ; \mathrm{dt}=0.00025 ; \operatorname{Re}=1892 ; \mathrm{Fr}=2.8$

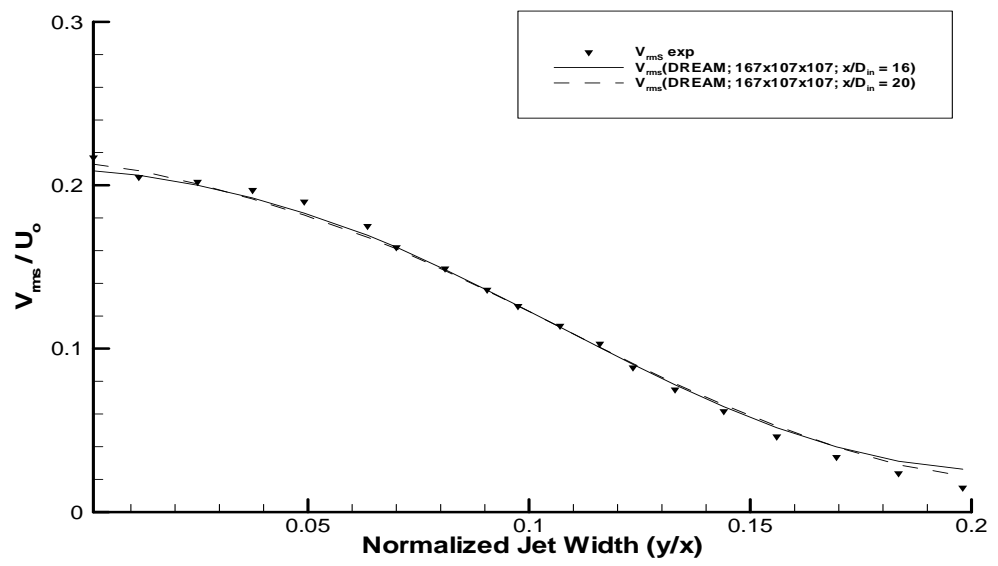

Figure 6.19 - Comparison of $V_{\text {rms }}$ from DREAM ${ }^{\circledR}$ simulations to experiments at $x / D_{\text {in }}=15$ and $x / D_{\text {in }}=20 ; d t=0.00025 ; \operatorname{Re}=1892 ; F r=2.8$

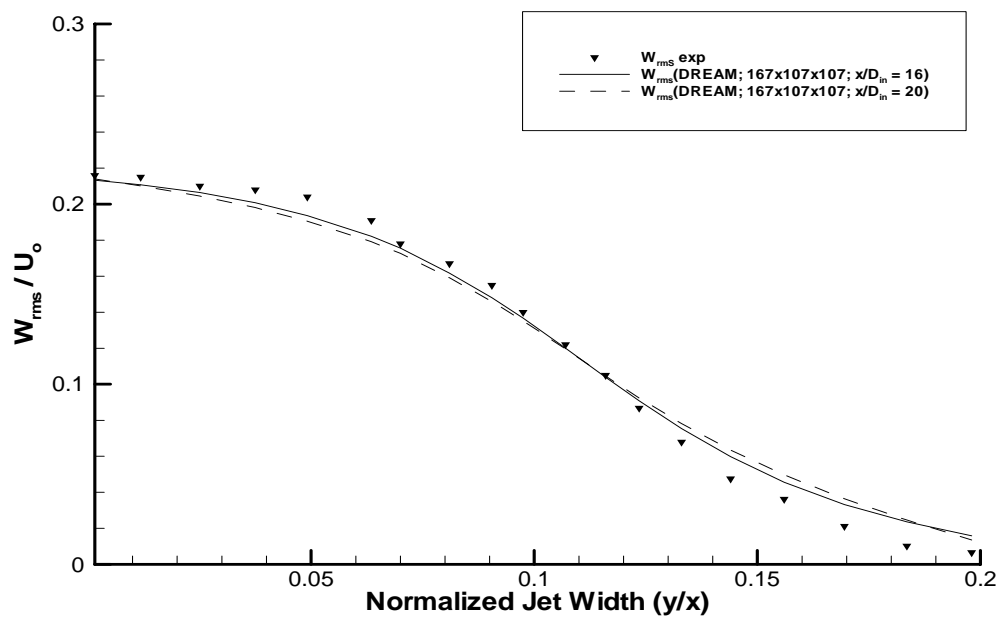

Figure 6.20 - Comparison of $W_{\text {rms }}$ from DREAM ${ }^{\circledR}$ simulations to experiments at $x / D_{\text {in }}=15$ and $x / D_{\text {in }}=20 ; \mathrm{dt}=0.00025 ; \operatorname{Re}=1892 ; \mathrm{Fr}=2.8$ 


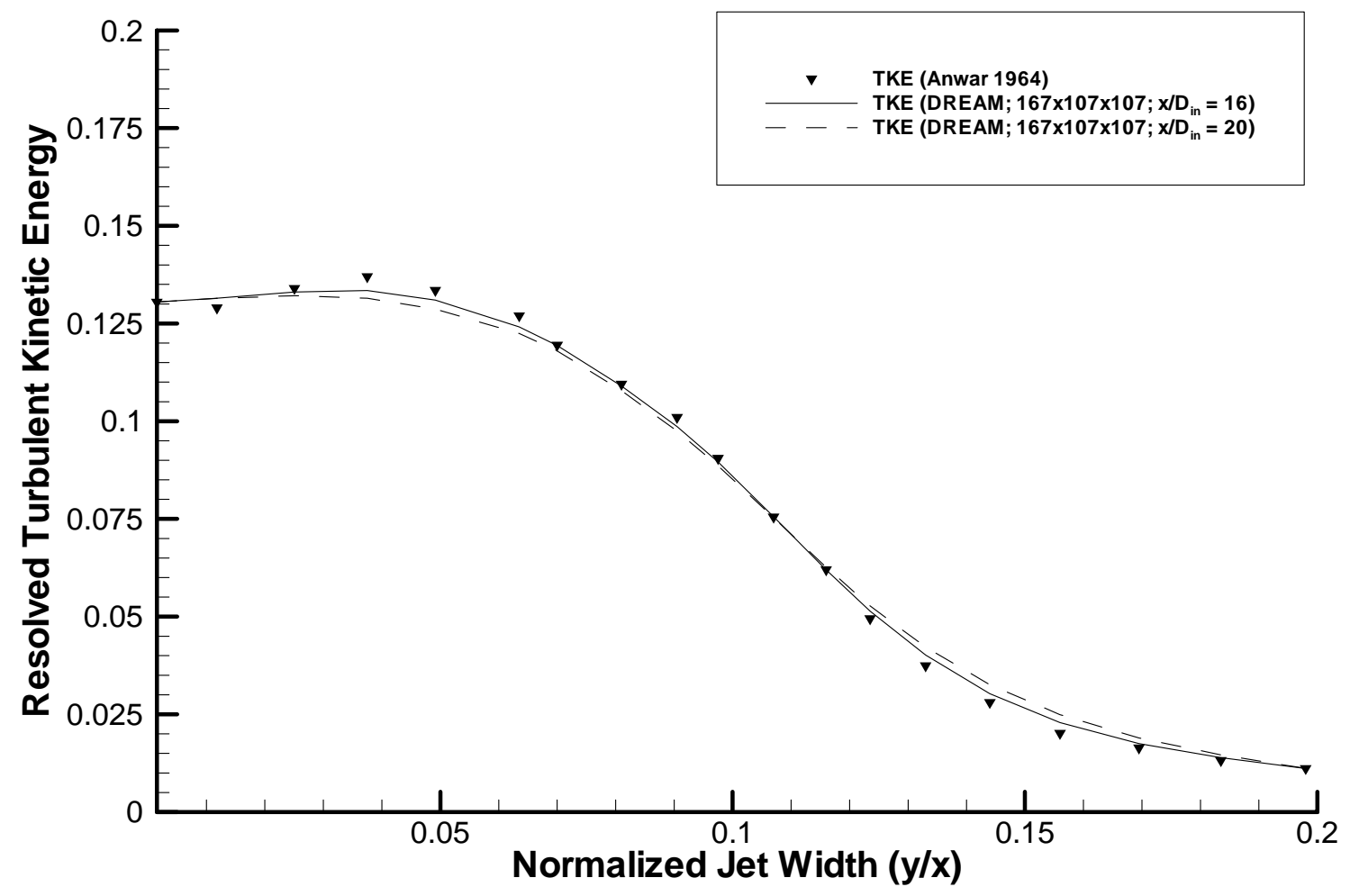

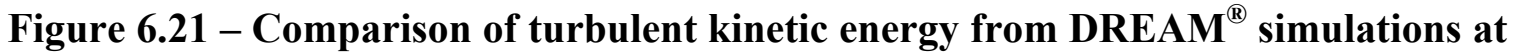
$x / D_{\text {in }}=15$ and 20 to experiments 


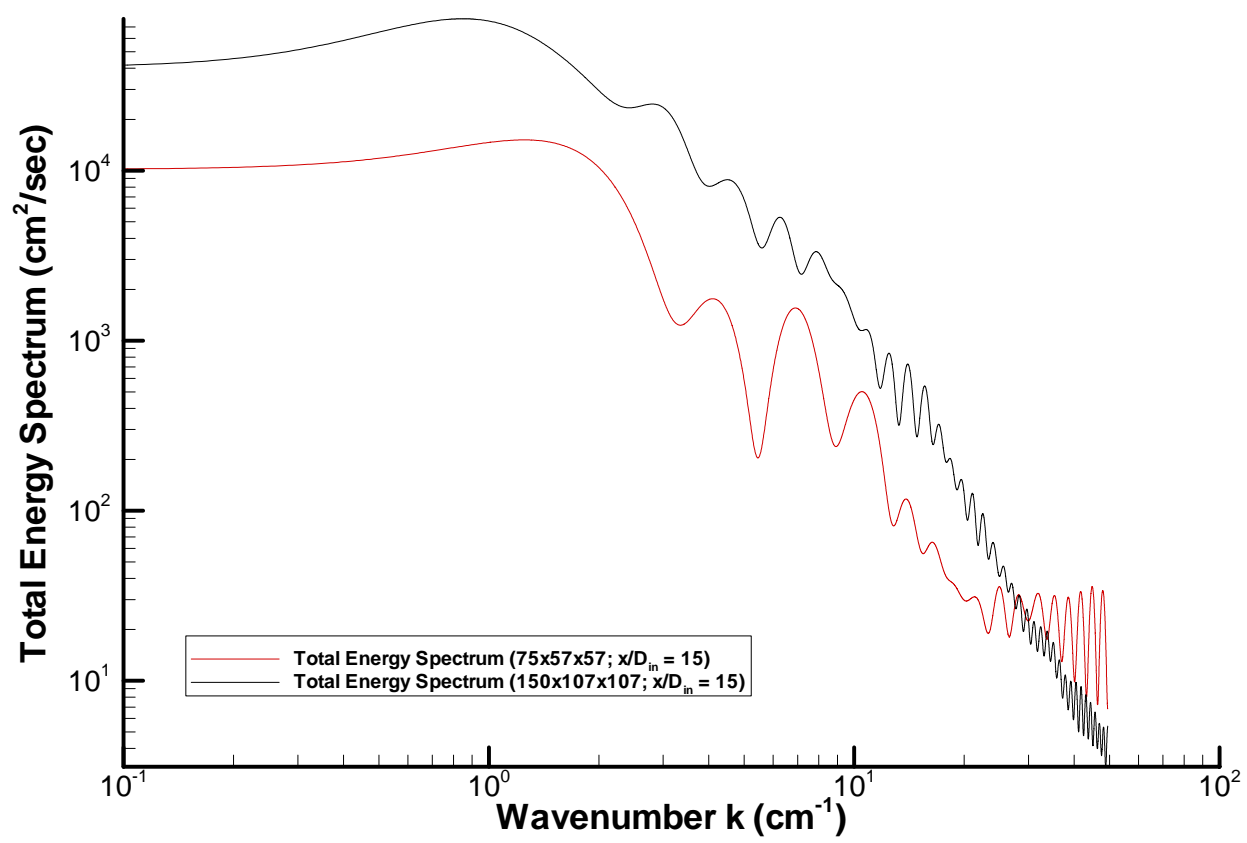

(a)

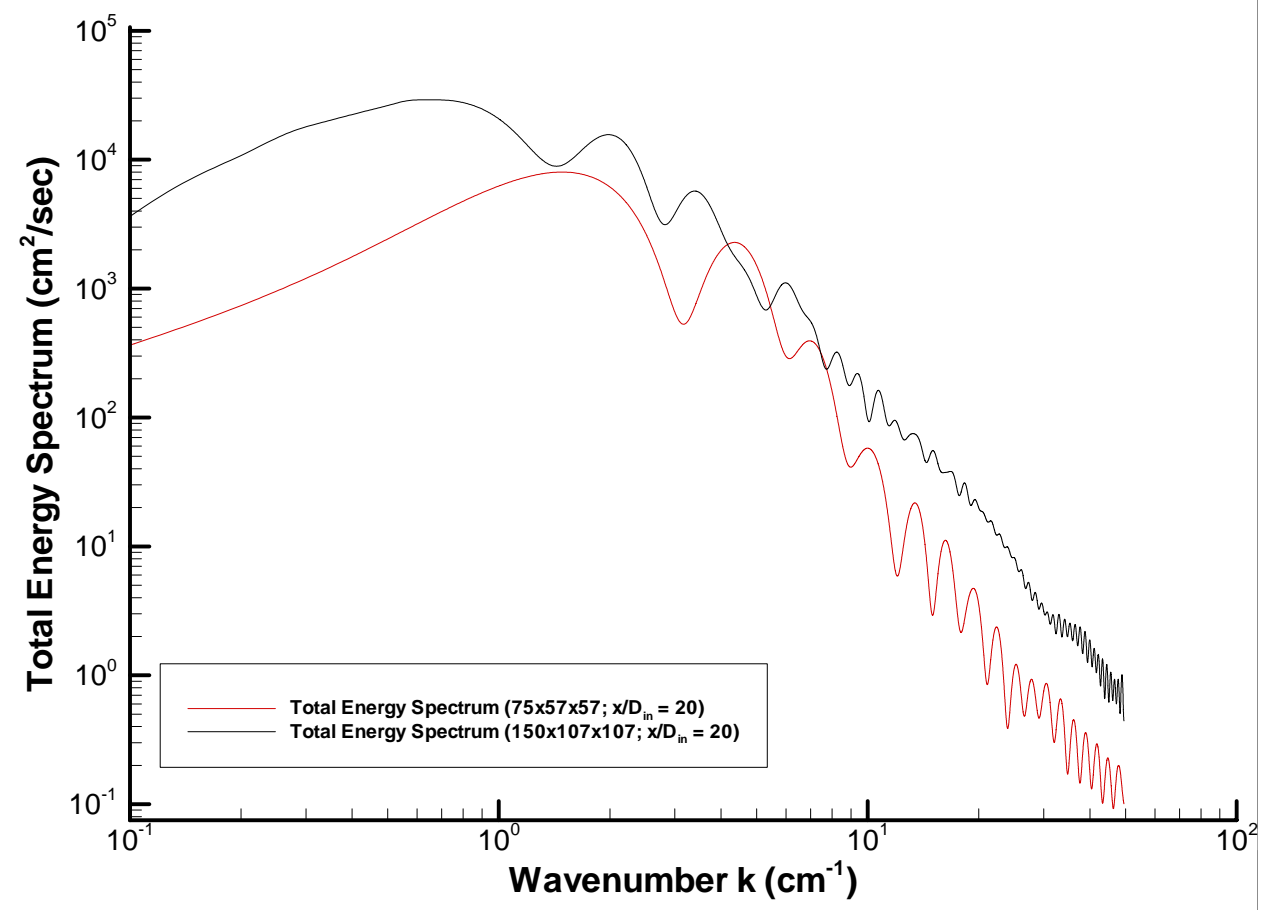

(b)

Figure 6.22 -a) Comparison of total energy spectrum at $\mathrm{x} / \mathrm{Din}=15$ from DREAM ${ }^{\circledR}$ simulations using coarse and fine grid simulations; b) Comparison of total energy spectrum at $x /$ Din $=20$ from DREAM ${ }^{\circledR}$ simulations using coarse and fine grid simulations 


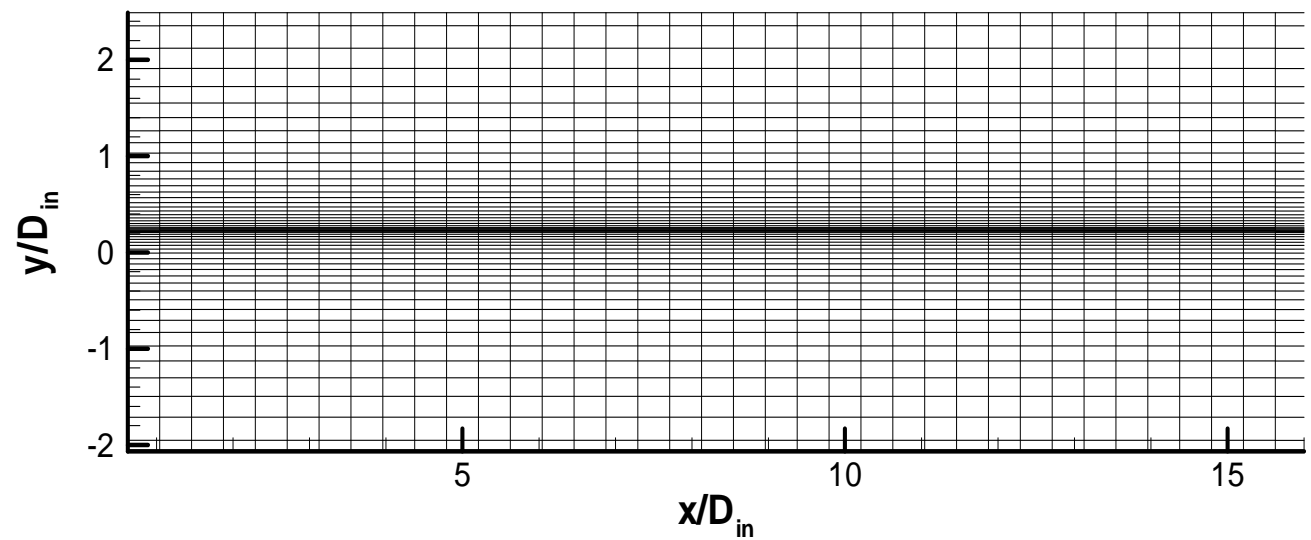

Figure 6.23 - Coarse grid used in DREAM ${ }^{\circledR}$ simulations for George and Tamanini (1977) experiments

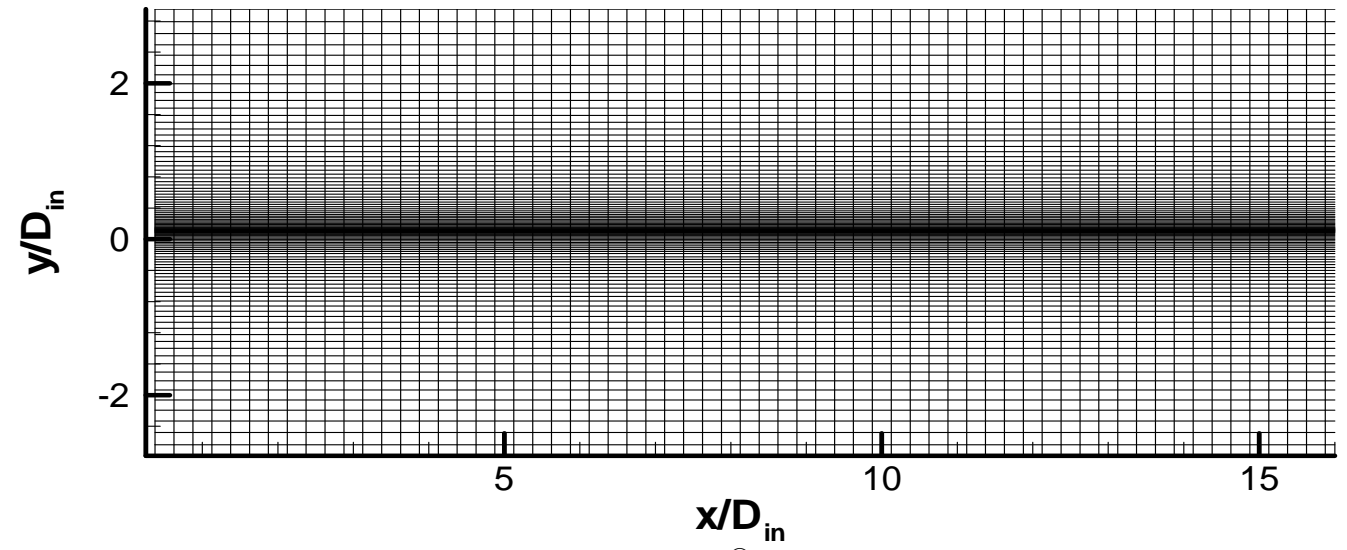

Figure 6.24 - Coarse grid used in DREAM ${ }^{\circledR}$ simulations for George and Tamanini (1977) experiments 


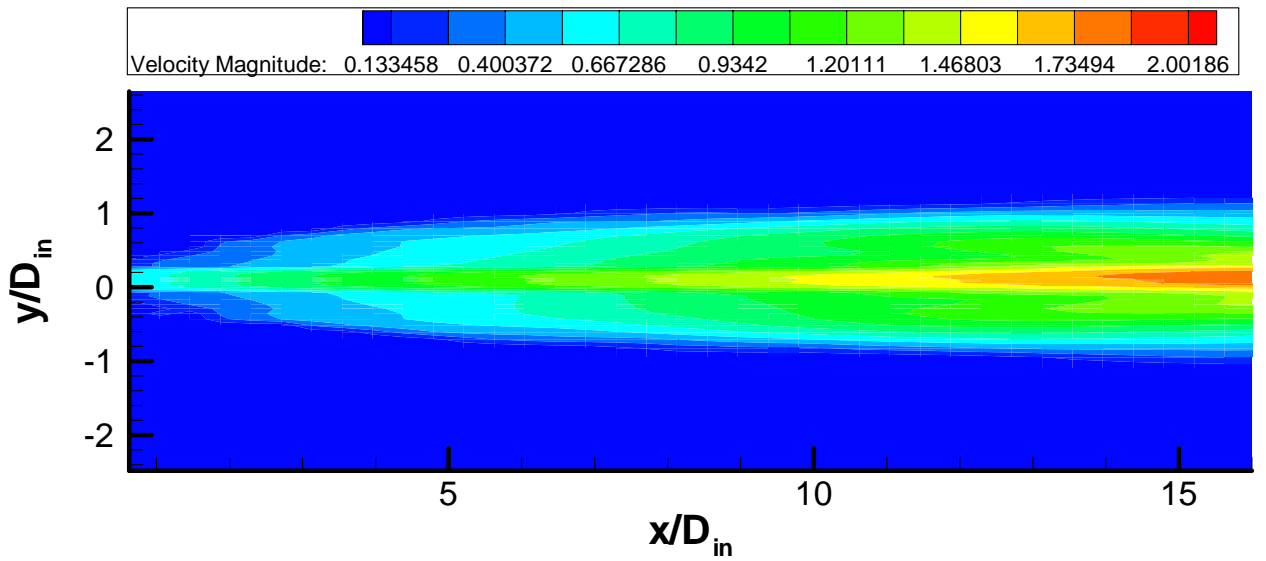

Figure 6.25 - Velocity magnitude contours at $\mathrm{z}=0$ for $75 \times 57 \times 57$ grid resolution after four flow through times; $\mathrm{dt}=0.00025 ; \operatorname{Re}=1273 ; \mathrm{Fr}=1.93$

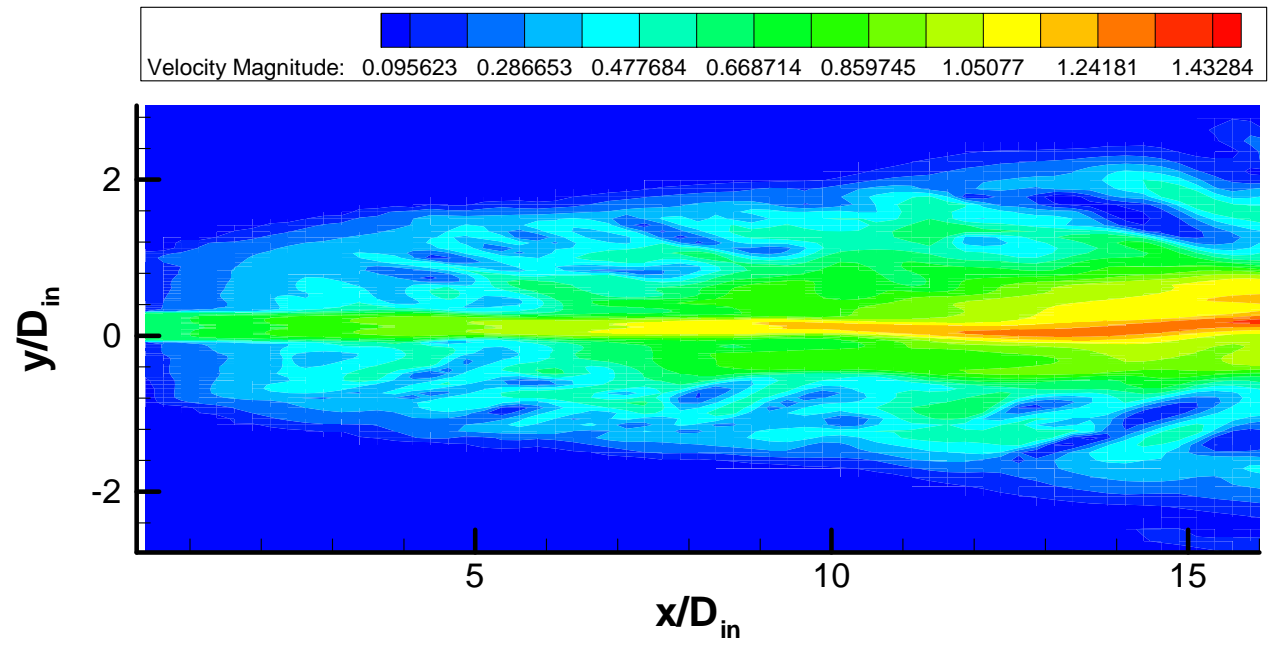

Figure 6.26 - Velocity magnitude contours at $z=0$ for $150 \times 107 \times 107$ grid resolution after four flow through times; $\mathrm{dt}=0.00025 ; \mathrm{Re}=1273 ; \mathrm{Fr}=1.93$ 


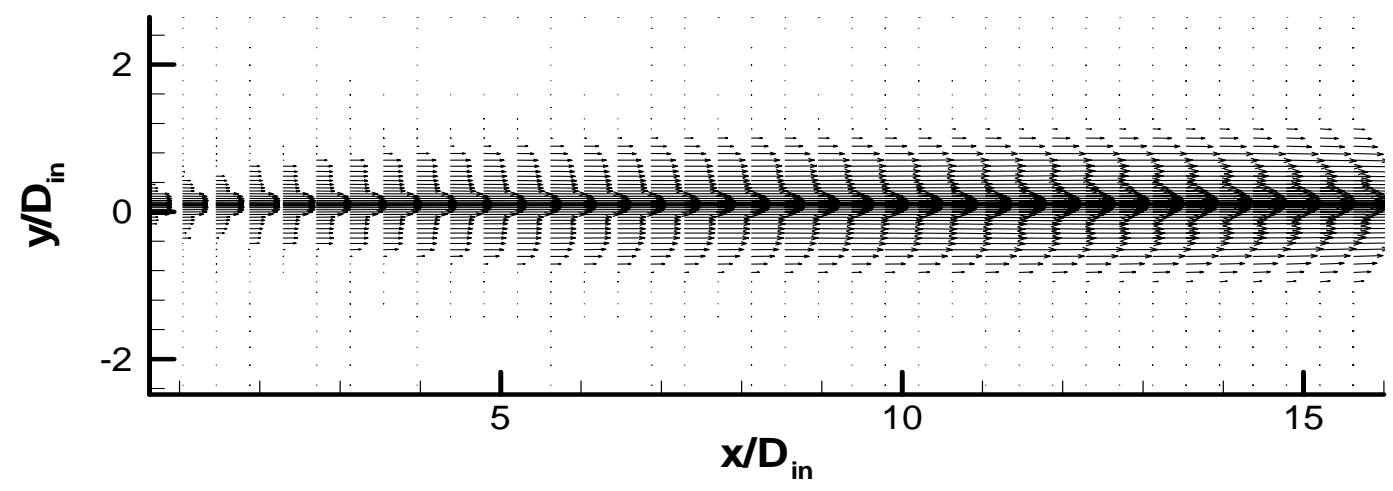

Figure 6.27 - Instantaneous velocity vectors at $\mathrm{z}=0$ for $75 \times 57 \times 57$ grid resolution after four flow through times; $\mathrm{dt}=0.00025 ; \operatorname{Re}=1273 ; \mathrm{Fr}=1.93$

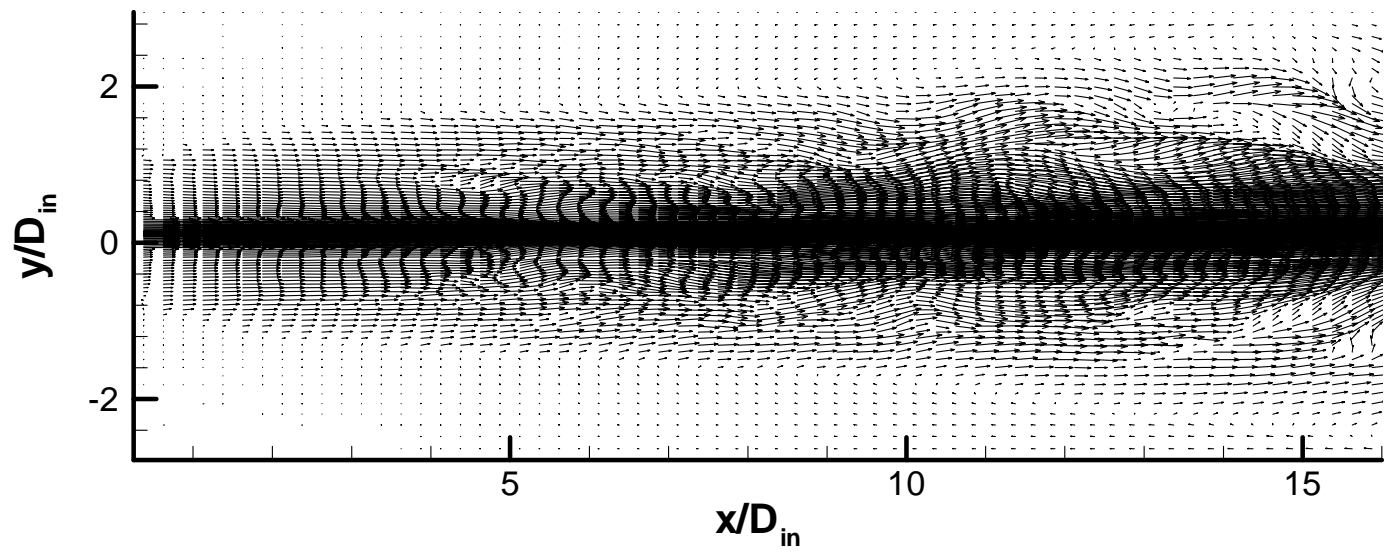

Figure 6.28 - Instantaneous velocity vectors at $\mathrm{z}=\mathbf{0}$ for $150 \times 107 \times 107$ grid resolution after four flow through times; $\mathrm{dt}=0.00025 ; \mathrm{Re}=1273 ; \mathrm{Fr}=1.93$ 


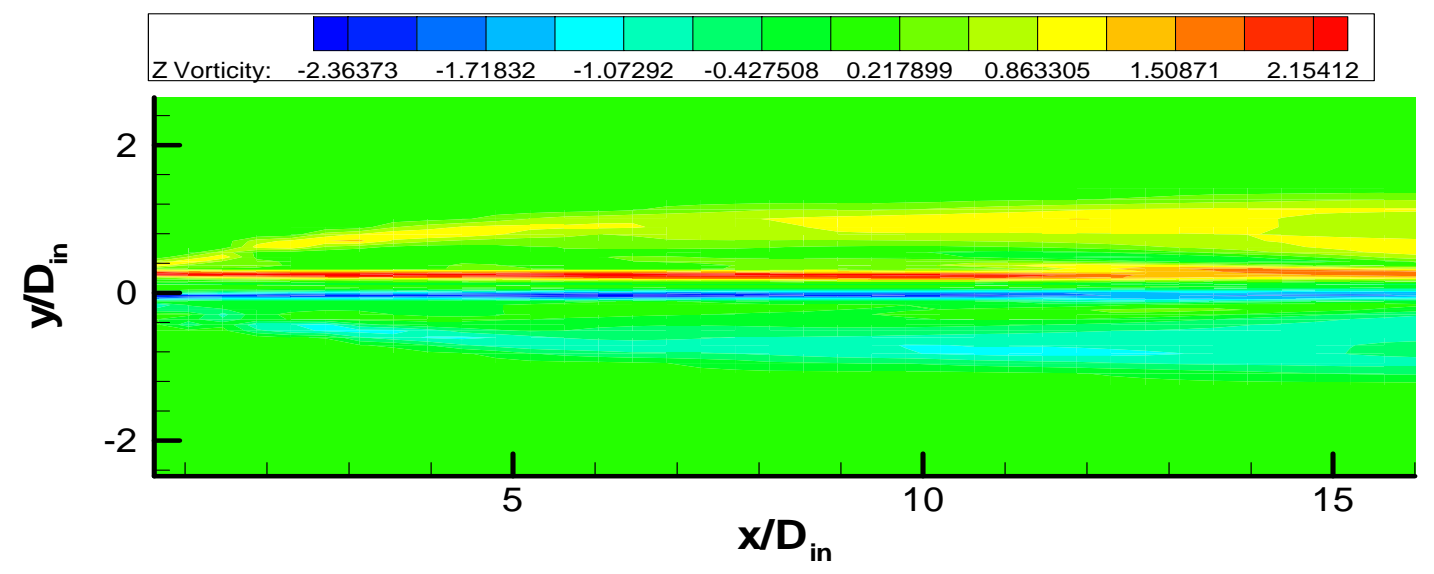

Figure $6.29-Z$ component vorticity contours at $z=0$ for $75 \times 57 \times 57$ grid resolution after four flow through times; $\mathrm{dt}=0.00025 ; \mathrm{Re}=1273 ; \mathrm{Fr}=1.93$

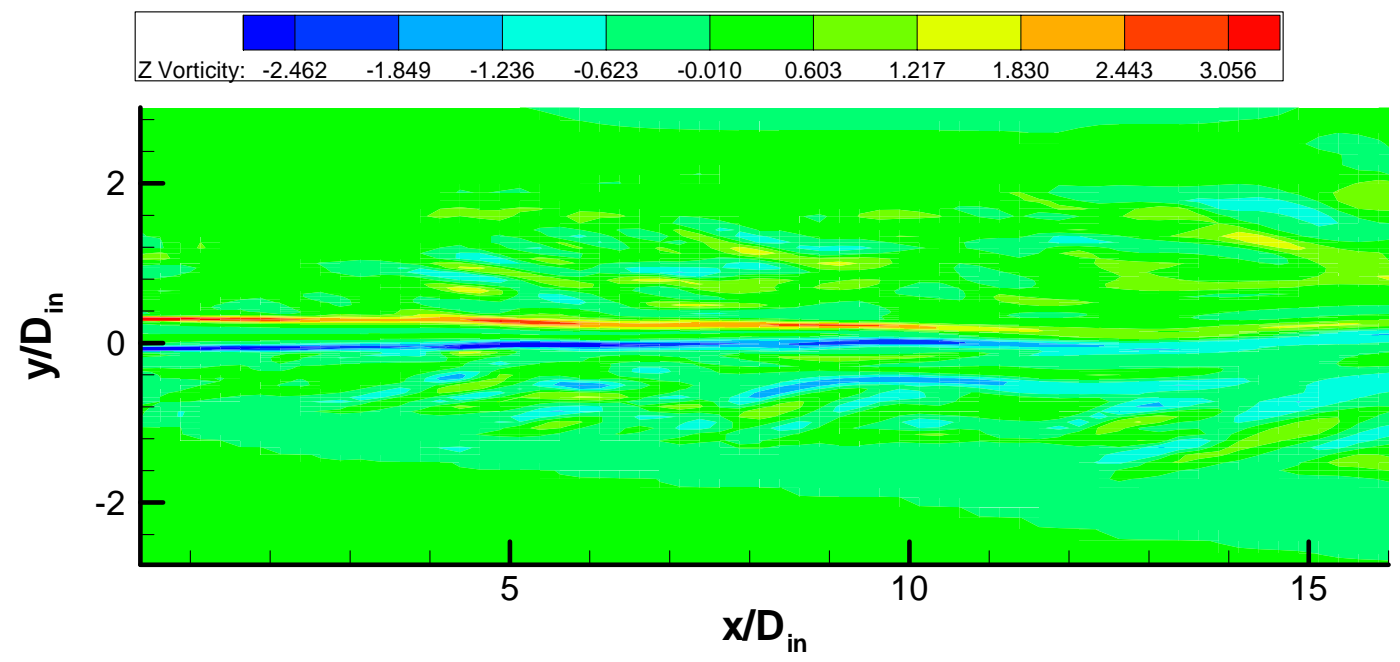

Figure 6.30 - $Z$ component vorticity contours at $z=0$ for $150 \times 107 \times 107$ grid resolution after four flow through times; $\mathrm{dt}=0.00025 ; \mathrm{Re}=1273 ; \mathrm{Fr}=1.93$ 


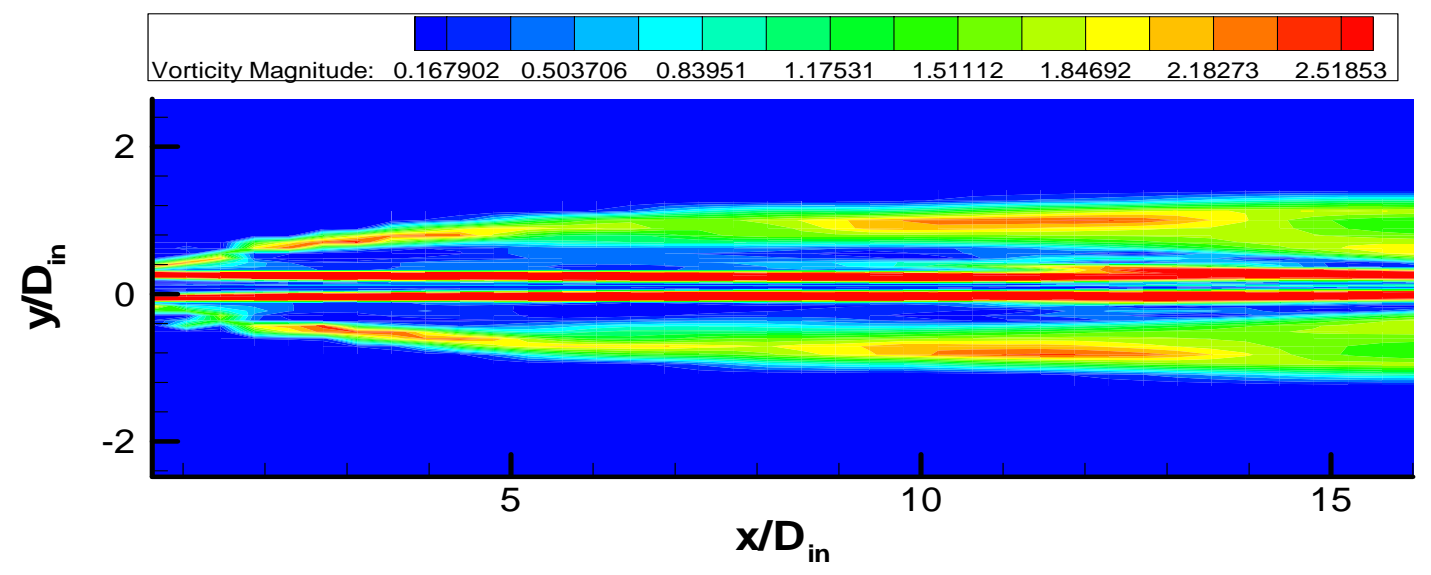

Figure 6.31 - Vorticity magnitude contours at $z=0$ for $75 \times 57 \times 57$ grid resolution after four flow through times; $\mathrm{dt}=0.00025 ; \mathrm{Re}=1273 ; \mathrm{Fr}=1.93$

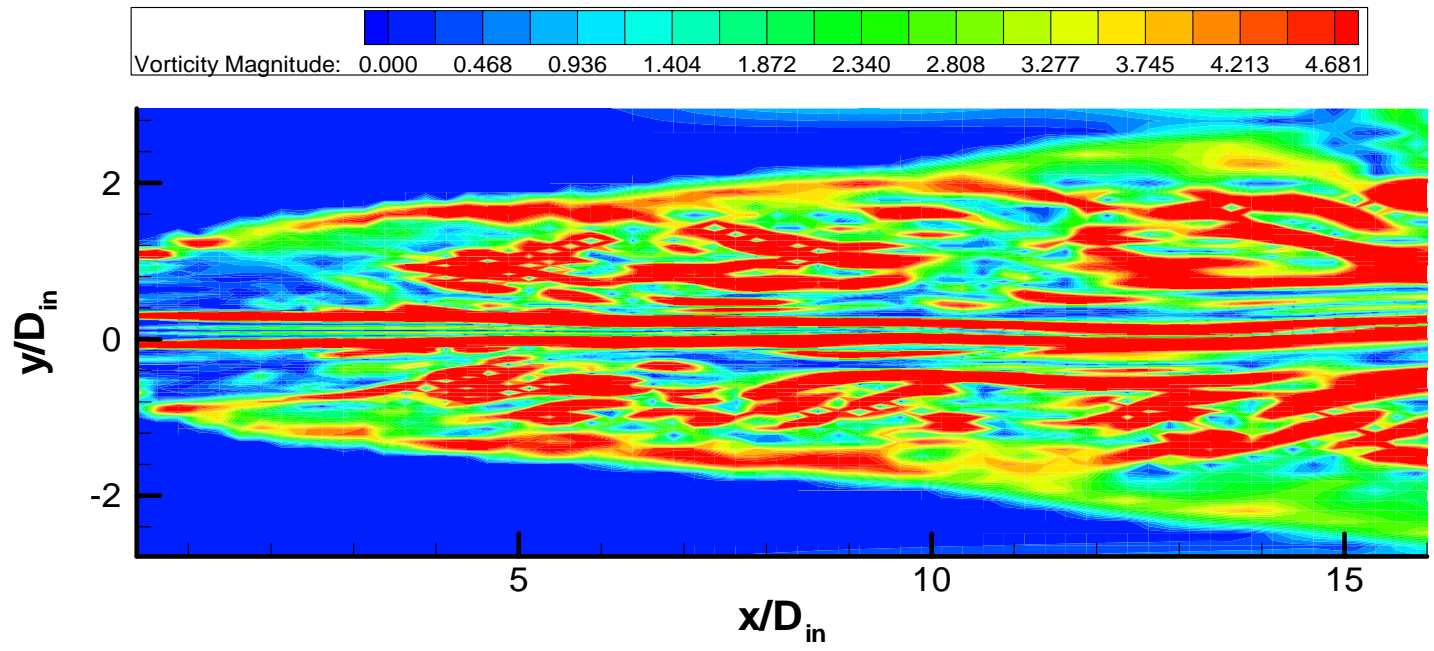

Figure 6.32 - Vorticity magnitude contours at $z=0$ for $150 \times 107 \times 107$ grid resolution after four flow through times; $d t=0.00025 ; \operatorname{Re}=1273 ; \mathrm{Fr}=1.93$ 


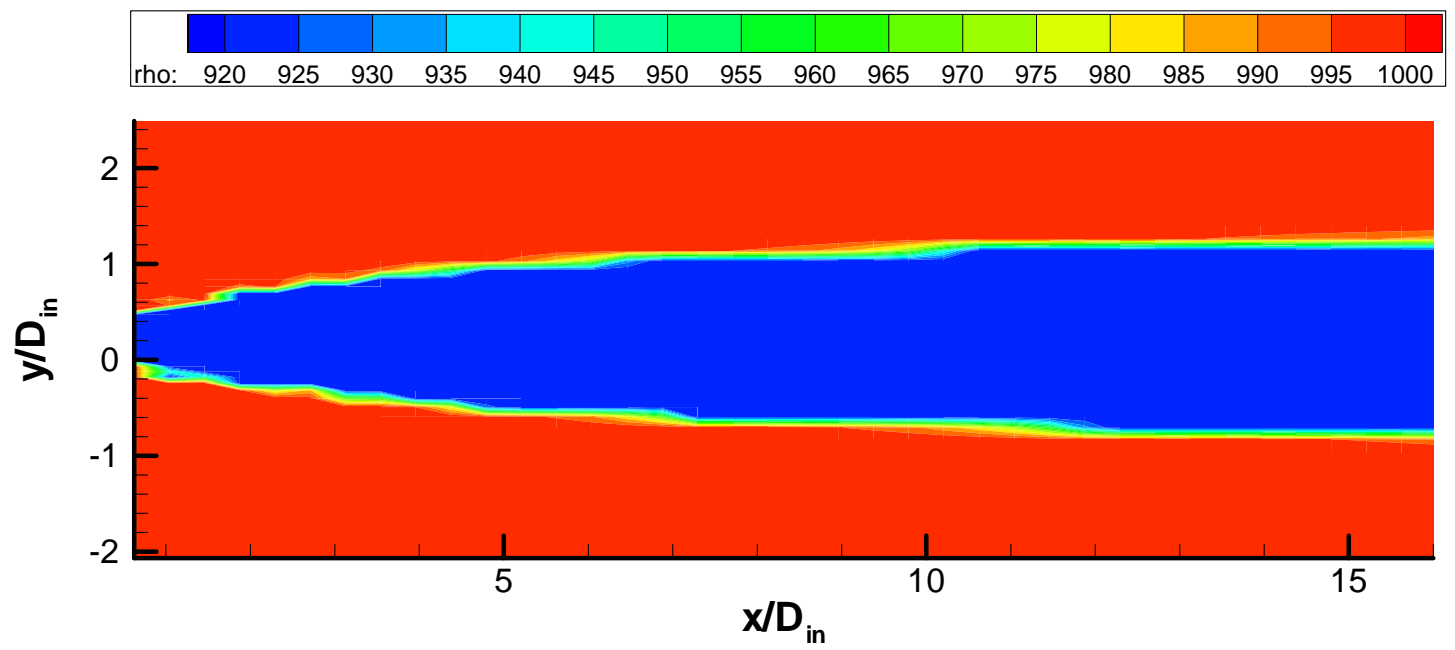

Figure 6.33 - Density contours at $\mathrm{z}=0$ for $75 \times 57 \times 57$ grid resolution after four flow through times; $\mathrm{dt}=0.00025 ; \operatorname{Re}=1273 ; \mathrm{Fr}=1.93$

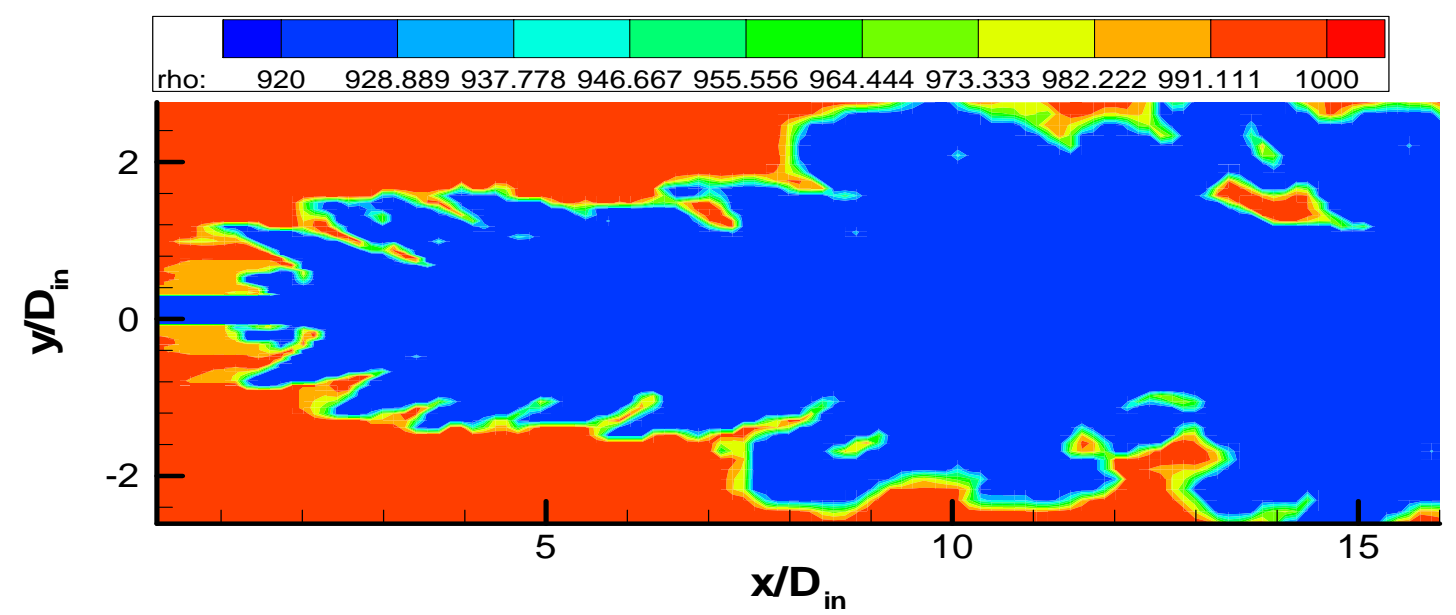

Figure 6.34 - Density contours at $z=0$ for $150 \times 107 \times 107$ grid resolution after four flow through times; $d t=0.00025 ; \operatorname{Re}=1273 ; \mathrm{Fr}=1.93$ 


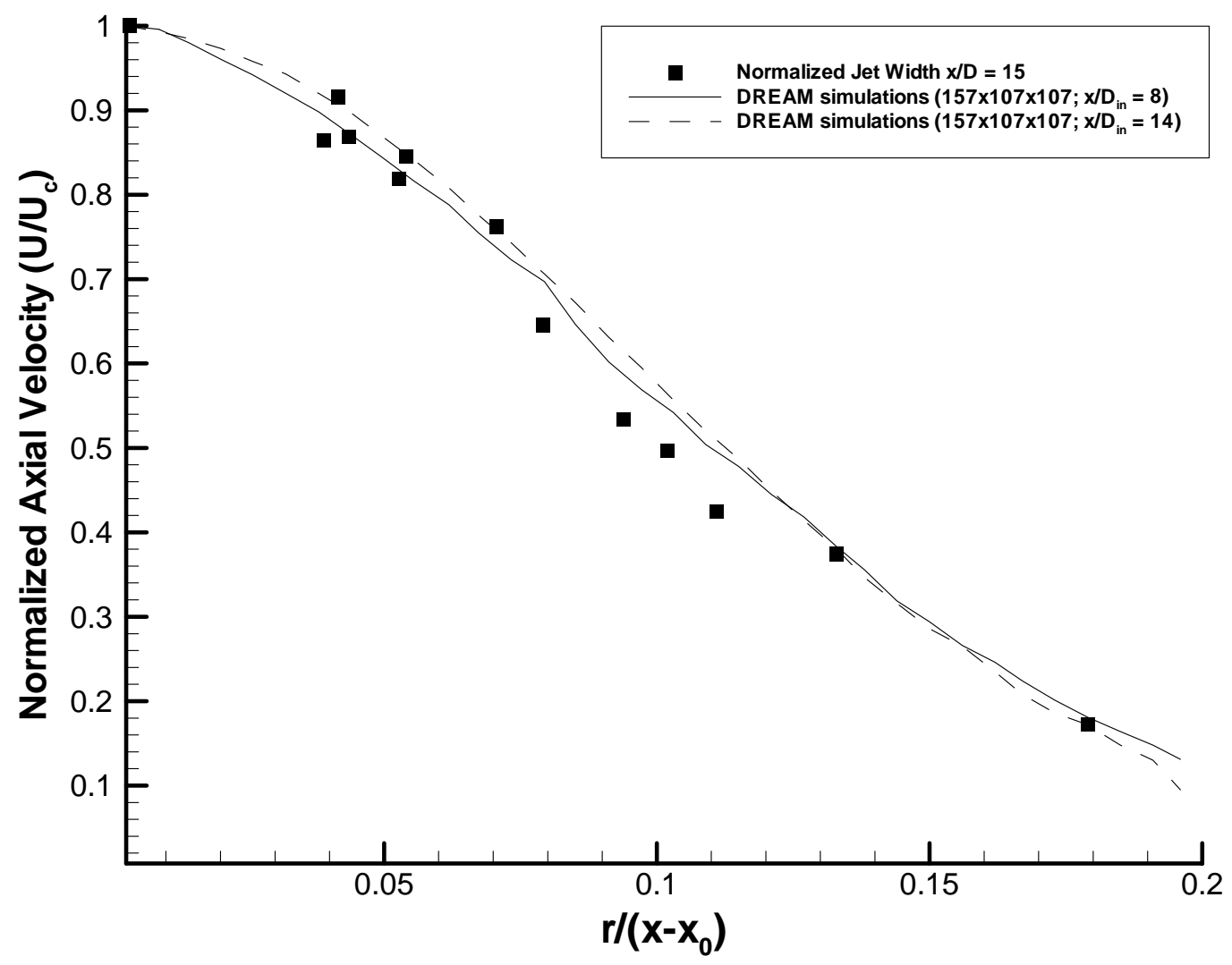

Figure 6.35 - Normalized axial velocity across the jet at $x / D_{\text {in }}=8$ and $x / D_{\text {in }}=14$ from DREAM ${ }^{\circledR}$ simulations as compared to experiments; $d t=0.00025 ; \operatorname{Re}=1273$; Fr $=1.93$ 


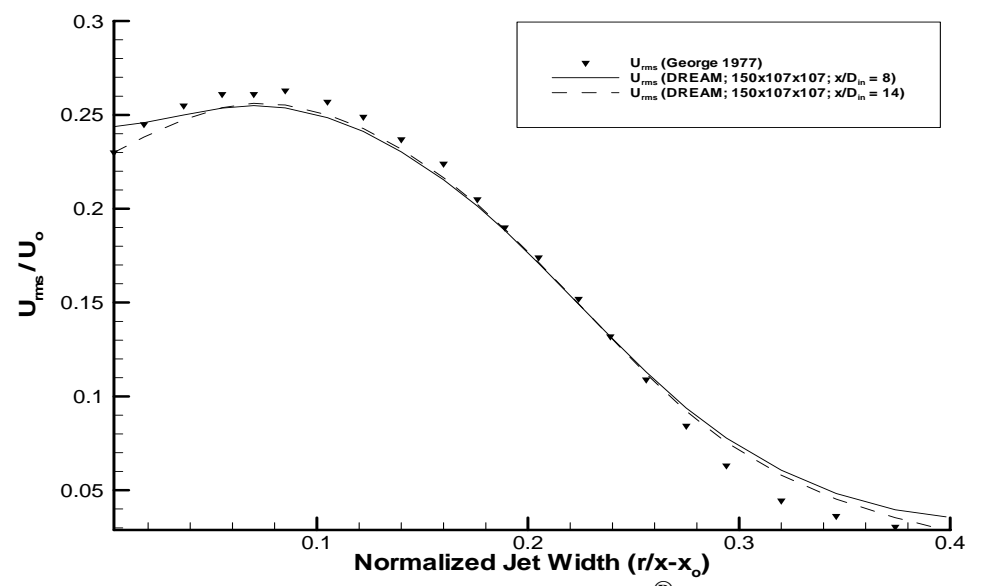

Figure 6.36 - Comparison of $U_{\text {rms }}$ from DREAM ${ }^{\circledR}$ simulations to experiments at $x / D_{\text {in }}=8$ and $x / D_{\text {in }}=14 ; d t=0.00025 ; \operatorname{Re}=1273 ; F r=1.93$

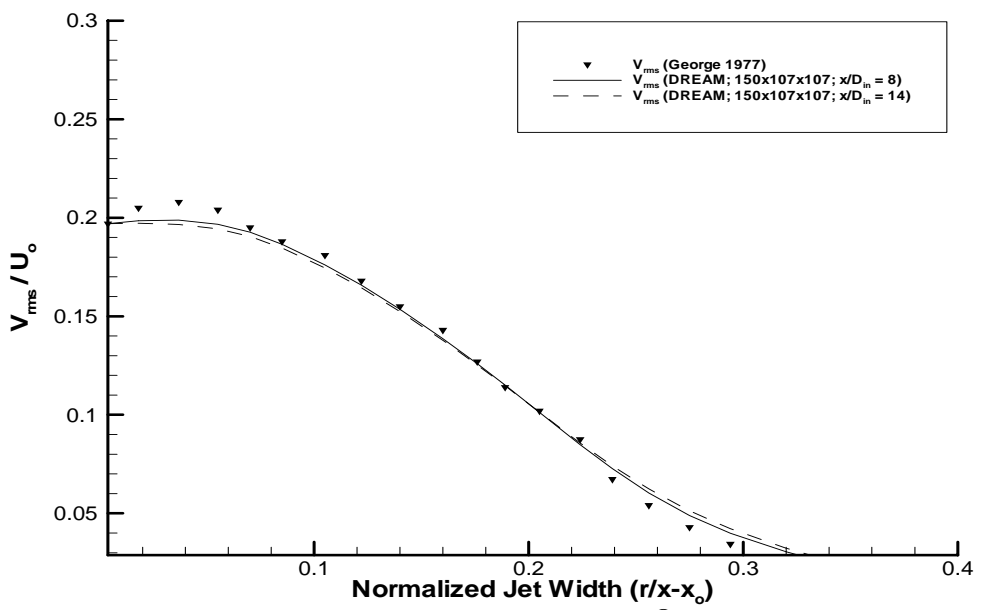

Figure 6.37 - Comparison of $\mathrm{V}_{\text {rms }}$ from DREAM ${ }^{\circledR}$ simulations to experiments at $x / D_{\text {in }}=8$ and $x / D_{\text {in }}=14 ; \mathrm{dt}=0.00025 ; \operatorname{Re}=1273 ; \mathrm{Fr}=1.93$

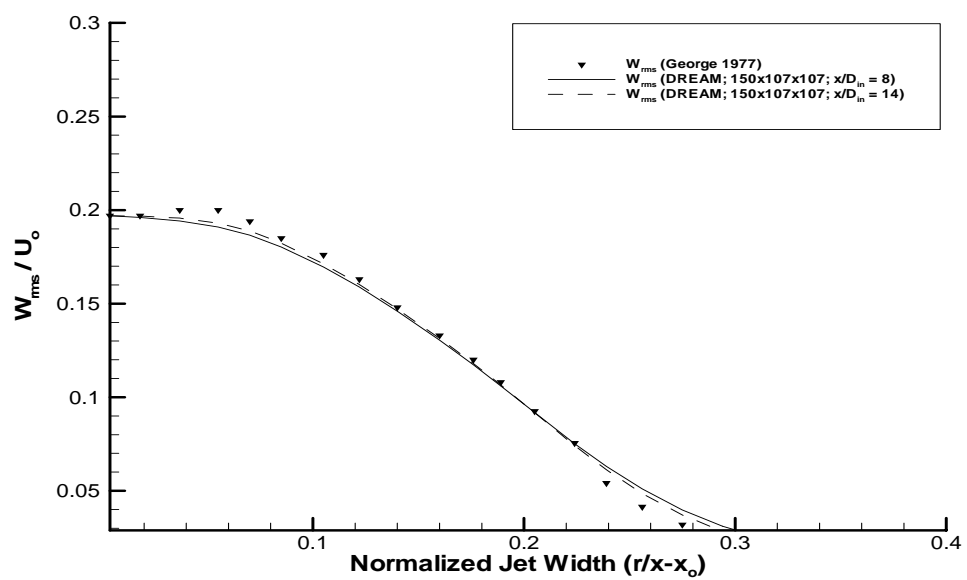

Figure 6.38 - Comparison of $W_{\text {rms }}$ from DREAM ${ }^{\circledR}$ simulations to experiments at $x / D_{\text {in }}=8$ and $x / D_{\text {in }}=14 ; \mathrm{dt}=0.00025 ; \operatorname{Re}=1273 ; \mathrm{Fr}=1.93$ 


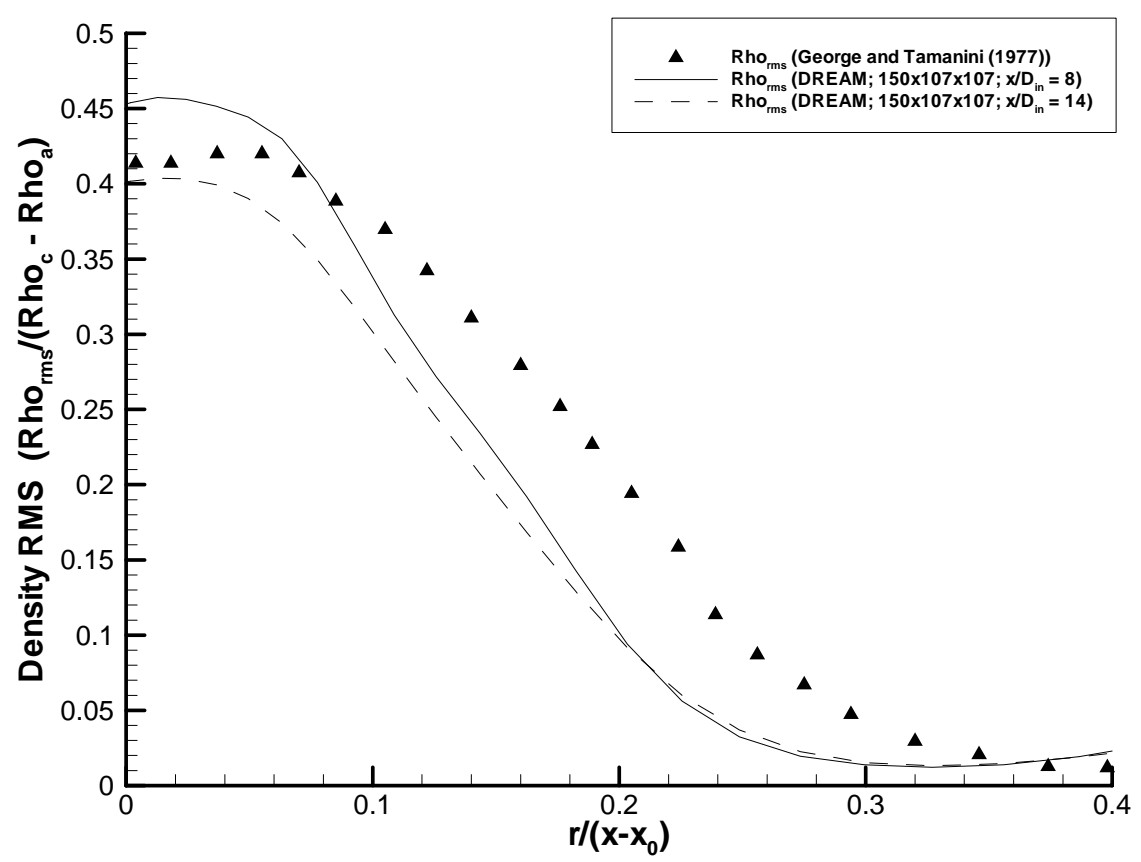

Figure 6.39 - Comparison of resolved density fluctuations from DREAM ${ }^{\circledR}$ simulations between to experimental data at $x / D_{\text {in }}=8$ and $x / D_{\text {in }}=14 ; \operatorname{Re}=1273 ; \mathrm{Fr}$ $=\mathbf{1 . 9 3}$

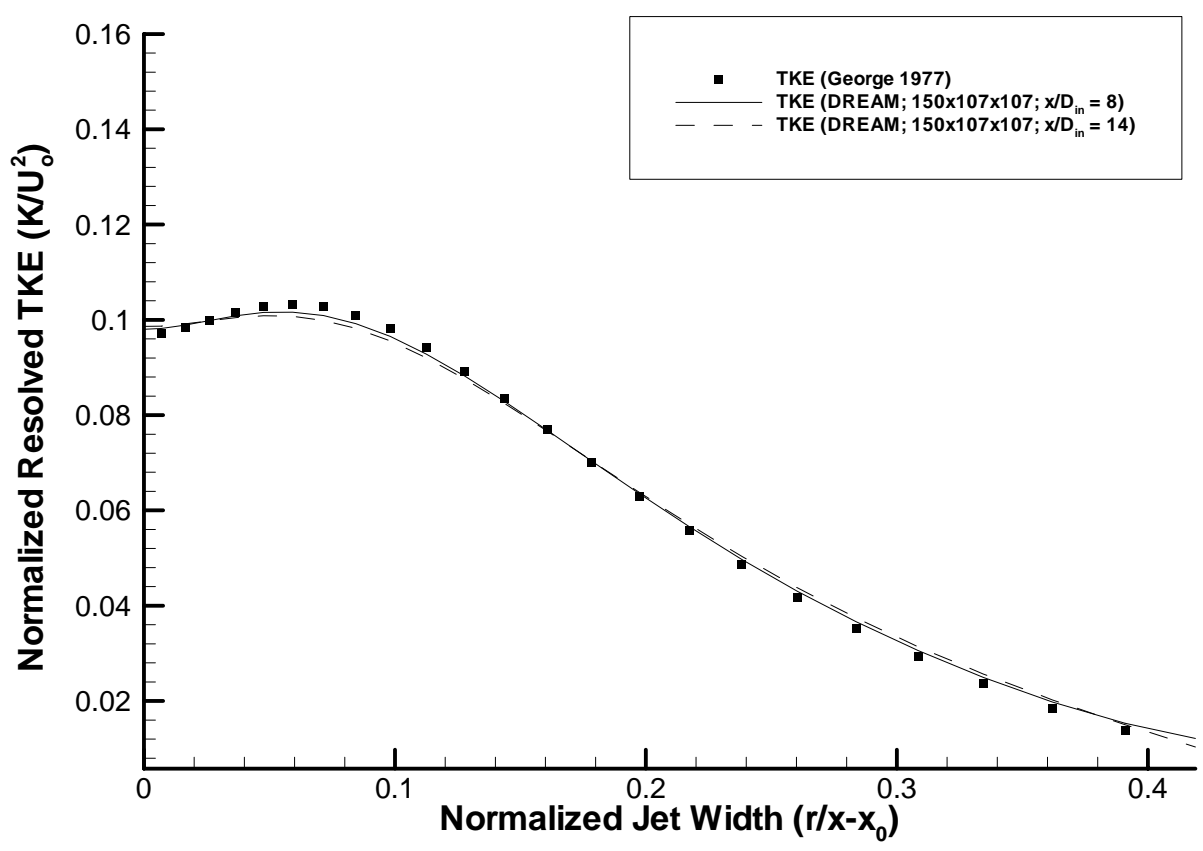

Figure 6.40 - Comparison of resolved turbulent kinetic energy from DREAM ${ }^{\circledR}$ simulations between to experimental data at $x / D_{\text {in }}=8$ and $x / D_{\text {in }}=14 ; \operatorname{Re}=1273 ; \mathrm{Fr}$ $=1.93$ 


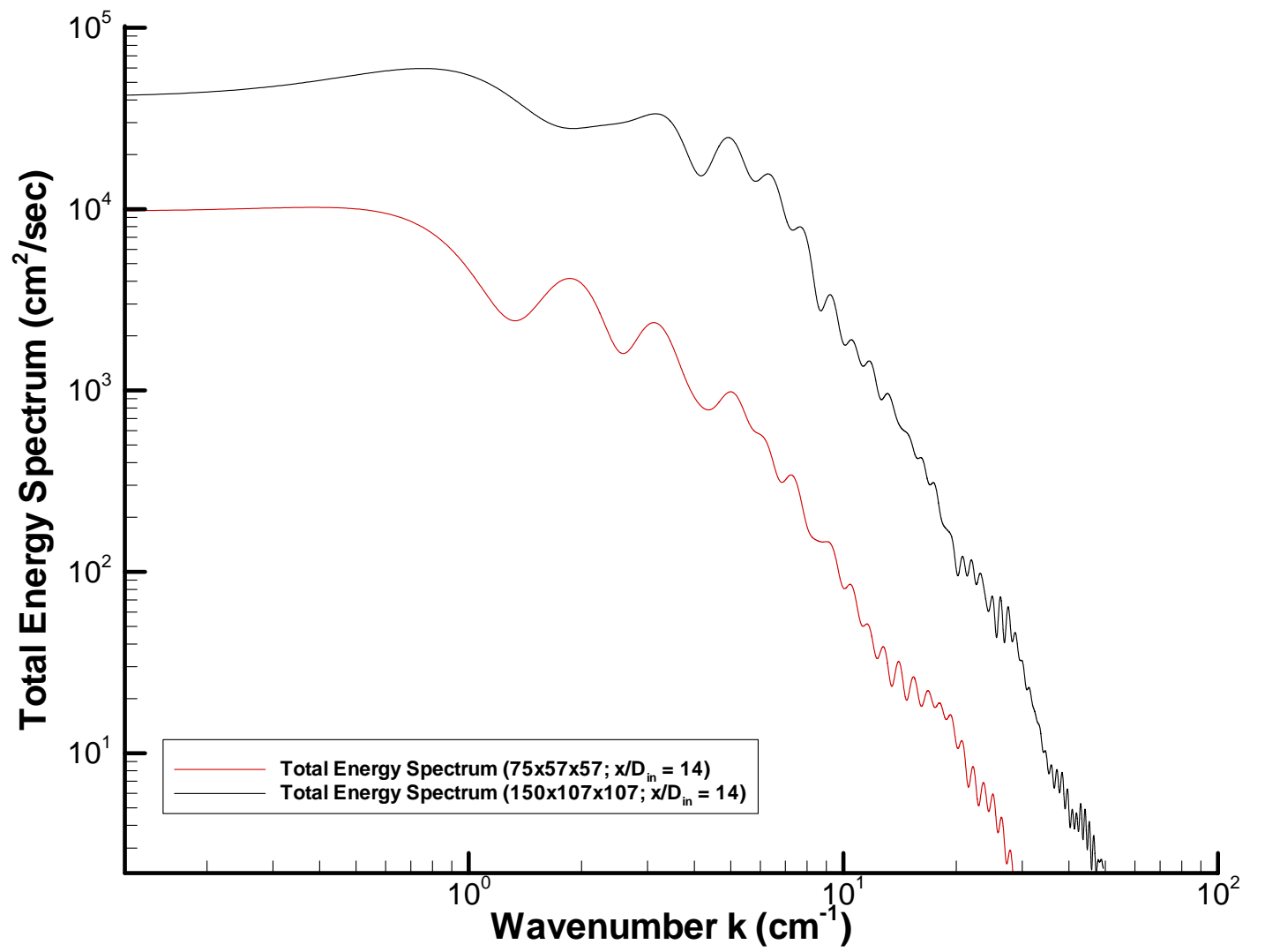

Figure 6.41 - Comparison of total energy spectrum at $x / D_{\text {in }}=14$ from DREAM ${ }^{\circledR}$ simulations using coarse and fine grid simulations; $\operatorname{Re}=1273 ; \mathrm{Fr}=1.93$ 


\subsection{CURVED BUOYANT JET}

\subsection{Introduction}

The problem of a sharp density interface impinged by a buoyant jet occurs in both natural and man-made situations. Examples include refueling of compensated fuel ballast tanks on naval vessels (Badeau, (2001); Wilson (1999)), aerosol injections in confined spaces, and ice covered thermal buoyancy driven flows. Again, it is important to note that the original reason for performing this study deals with the buoyant flow events that ensue during the refueling process within the compensated fuel ballast tanks. Impingement behavior also encompasses the impacting of buoyant jets onto solid surfaces, which still has received little attention, as most studies investigate the resulting flows after impaction. The behavior of a jet injected horizontally into a stagnant liquid of different density results in a path curvature, which is dependent on the momentum and buoyancy of the jet. Initially, the immiscible liquid impingement results in the development of a horizontal cavity, which travels based on buoyancy and momentum forces. As the flow phenomenon occurs within an enclosure, the behavior near the wall also effect the turbulence within the chamber, as well as the long term behavior of the jet. A variety of dimensionless parameters may be used to describe the ensuing flow field; however, a densimetric Froude number or Reynolds number based on the inlet properties are utilized for this study. Experiments have been performed in conjunction with the computational studies to help in the quantitative analysis of the $\operatorname{DREAM}^{\circledR}$ simulations. This chapter examines critical components of the curved buoyant jet and the long term flow field characteristics utilizing LES and ITM methods. The impingement of the curved buoyant jet onto the solid wall also is investigated.

\subsection{Experiments}

The experiments presented in this section were explicitly performed by the author of this study to determine the centerline path and jet width of the curved buoyant jet as a method of validation of the CFD simulations from DREAM ${ }^{\circledR}$. Although other simulations are similar in manner to the ones explained in this section, the available 
experimental data specifically dealing with only a curved buoyant jet flowing into a stagnant fluid were lacking. The experiments were conducted by the author over a onemonth time period, which does not included building and design of the experiment. The experimental setup, shown in Figure 7.1, consists of a water jet, injected horizontally at three different flow rates, into a stagnant corn oil. Tap water at city pressure and density of $999.91 \mathrm{~kg} / \mathrm{m}^{3}$ was utilized for the experiment, with generic corn oil, at a density of 920 $\mathrm{kg} / \mathrm{m}^{3}$. As the numerical simulations will have corn oil injected into water, densimetric Froude number similarity will be used to determine the appropriate water inlet velocities. The key parameters used in the experiments are summarized in Table 7.2.1. The exit densimetric Froude number based on the diameter of the inlet pipe was used to determine all densimetric Froude numbers.

The inner dimensions of the tank used in the experiments were $0.6 \times 0.4 \times 0.2 \mathrm{~m}$, with the inlet hole consisting of a square of sides 0.02 meters in length, located in the center of the inlet plane. The chamber is constructed of half-inch LEXAN $^{\odot}$, with the inner edges bonded using acetone weld. A thin right angle strip of $\operatorname{LEXAN}^{\odot}$ is placed on the outside edges to help in the prevention of leaking. The outlet is offset 0.16 meters from center, which prevents symmetry instabilities that often result when symmetric geometries are used. Another outlet is available for simulations; however, for the current research, it is only used as a water drain during the refilling process and is closed during all experiments. Shutoff valves are at all inlets and exits, as well as a plug being used for the refilling location of the oil. For all experiments, water fills the tank to the one-quarter capacity, with a collection tank being used for the exiting oil. The inlet water velocity is controlled using a Cole-Parmer ${ }^{\odot}$ flow meter, which was calibrated through water filling experiments to determine the appropriate velocities at the inlet.

The goal of the experiments is to help in the validation of the DREAM ${ }^{\circledR}$ simulations. The properties of the curved buoyant are investigated. These results are compared to the DREAM $^{\circledR}$ simulations in section 7.4. To acquire the data, two digital video cameras are placed at the center bottom and left center locations, being at ninety degree angles to the view planes. These locations are chosen to make the jet analysis easier. Also, background lighting from a standard flow lamp is utilized to help ensure 
that enough light is provided for the camera. The movies are imported into Adobe Premier $^{\odot}$, a movie rendering software package, which allows for instantaneous pictures to be extracted from the movies and converted to an appropriate format suitable for MATLAB $^{\odot}$ analysis, i.e. TIFF, JPEG, etc.

To determine the jet width and centerline locations, a MATLAB ${ }^{\odot}$ function called "edge (sobel)" was used, which allows the edges to be identified based on a given threshold, with an uncertainty of $0.2 \%$ due to pixel limitations. Unfortunately, because the curved jet is in the center of the tank to avoid wall effects and the cameras are outside of the enclosure, other flow field characteristics are difficult to analyze and are not presented in this study. The threshold was determined through trial and error and is varied to determine the jet width and centerline locations. The Sobel method finds edges using the Sobel approximation to the derivative. For details on the Sobel method, the reader is referred to Matlabs documentation on image processing, which may be found in MatlabV7 (2002). The Sobel method returns edges at those points where the gradient of I is maximum based on the defined threshold. An example of the coding used for this function is given by

$$
\text { jet }=\text { edge(I,'sobel',thresh) }
$$

\section{jet_center $=$ edge $(I$, 'sobel',thresh,direction $)$}

where the direction is an array keeping track of the $\mathrm{x}$ and $\mathrm{y}$ locations for the jet centerline tracking. This data is then exported to a data file for graphing. Results are presented in Section 7.4 for comparison to the DREAM ${ }^{\circledR}$ simulations. A total of 16 experiments were performed for each densimetric Froude number, and the results were averaged over all the runs to obtain the jet trajectory. It is important to note that the two lower Froude number experiments performed for this study have similar dimensionless quantity magnitudes as compared to the experiments pertaining to the compensated fuel ballast tank experiments (Wilson, 1999). The maximum variations in the experiments between each of the runs for each Froude number are also compared to the simulation results presented in Section 7.4. 


\begin{tabular}{|l|l|l|l|l|l|l|}
\hline Fluid & $\mathbf{D}(\mathbf{m})$ & $\mathbf{F r}$ & $\mathbf{1} / \mathbf{F r}$ & $\mathbf{R e}_{\text {jet }}$ & $\mathbf{U}_{\text {in }}(\mathbf{m} / \mathbf{s e c})$ & $\mathbf{t}_{\text {fill } 1 / 4}(\mathbf{s e c})$ \\
\hline water-oil & 0.02 & 1.91 & 0.52 & 4890 & 0.24 & 120 \\
\hline water-oil & 0.02 & 3.83 & 0.26 & 9902 & 0.48 & 60 \\
\hline water-oil & 0.02 & 7.66 & 0.13 & 19804 & 0.96 & 30 \\
\hline
\end{tabular}

Table 7.2.1 - Key parameters for buoyant jet experiments

\subsection{Computational Details}

The simulations of the curved buoyant jet, resulting from the injection of oil at a constant velocity into a stagnant water tank, are the primary focus of this study. The simulations are identical, in dimensions, to the experimental apparatus as described in section 7.2. densimetric Froude number similarity is maintained for all simulations.

For this study, three different grid resolutions were utilized, being 120x60x40, 180x120x60, and 240x160x80 in the x, y, and $\mathrm{z}$ directions, respectively. This yielded a total number of $0.288,1.296$, and 3.072 million grid cells, respectively. The coarsest grid is only used in the Richardson extrapolation analysis to determine approximate grid independence of the simulations, and other coarse grid results are not presented in this work. For all simulations, a constant averaged fuel droplet size of $0.5 \mathrm{~mm}$ was specified for the calculation of the slip velocity. A time step of $\mathrm{dt}=0.001$ was used for all simulations. A uniform grid was utilized for all simulations, with the grid sizes being approximately 300, 150 and 50 times the approximate viscous length scale. The coarsest grid is only used to test for an independent solution, and is not used in any other analysis.

The experiments use an inlet water jet for convenience, impinging onto a stagnant fuel layer, which causes a curved jet in the downward direction. To attain densimetric Froude number similarity, the inlet velocities are modified to ensure that the densimetric Froude numbers are equal in all cases studied. However, Reynolds number similarity is not maintained between the experiments and simulations. Thus, for the DREAM ${ }^{\circledR}$ simulations, the velocities used are given below in Table 7.3.1, along with the theoretical tank filling times. However experiments only last one-quarter of the theoretical tank 
filling time to conserve resources. For comparative purposes, a dimensionless time, $\mathrm{t}^{*}$, is utilized for characterization of the instantaneous profiles, which equals the instantaneous computational time divided by the total filling time.

\begin{tabular}{|l|l|l|l|l|l|l|}
\hline Fluid & $\mathbf{D}(\mathbf{m})$ & $\mathbf{F r}$ & $\mathbf{1 / F r}$ & $\mathbf{R e}_{\text {jet }}$ & $\mathbf{U}_{\text {in }}(\mathbf{m} / \mathbf{s e c})$ & $\mathbf{t}_{\text {fill }}(\mathbf{s e c})$ \\
\hline water-oil & 0.02 & 1.91 & 0.52 & 5000 & 0.25 & 480 \\
\hline water-oil & 0.02 & 3.83 & 0.26 & 10000 & 0.5 & 240 \\
\hline water-oil & 0.02 & 7.66 & 0.13 & 20000 & 1.0 & 120 \\
\hline
\end{tabular}

Table 7.3.1 - Key parameters for buoyant jet simulations

At the wall boundaries a no-slip velocity condition was applied. No wall functions are used in the simulations. In both experiments and the simulations, the inlet is a square, and analysis of the dimensionless parameters uses a hydraulic diameter. Since one of the key parameters in determining similarity is the location whereby the square jet resembles a circular jet, the inlet shape is of little importance for this analysis.

\subsection{Results and Conclusions}

This section shows the results from both the DREAM ${ }^{\circledR}$ simulations and the experiments. The curved buoyant jet properties are analyzed in Section 7.4.1. The similarity properties across the jet are examined, and compared to the available experimental data for non-buoyant jets. Other flow field properties within the jet region are also examined, such as the mean axial velocity across the jet, and the turbulent kinetic energy present within the jet. The jet centerline path for different densimetric Froude numbers is examined, as well as differences between the simulations and experiments for the highest Froude number case. In Section 7.4.2, the effects of the curved buoyant jet on the global flow field is studied to determine the flow field properties within the enclosure. The jet impingent on the solid wall, and the effects on the turbulence generation are also presented. 


\subsubsection{Curved Buoyant Jet Properties}

The most commonly studied turbulent free shear flows are jets. As implied, free jet implies that no walls effects are introduced, and the turbulence within the flow arises solely from the velocity fluctuations produced under the action of mean flow gradients. The flow is governed mostly by the Reynolds number based on the inlet velocity and inlet pipe diameter. For flows with a jet injected into an ambient, identical liquid at rest, usually a flat topped velocity profile is produced at the inlet. The flow is statistically stationary and axisymmetric (Pope, 2000). For these types of flows, buoyancy forces have no effect on the interactions between the two fluids, which is not the case for the simulations presented in this study; however, both types of flows exhibit similar characteristics within the jet region itself. This section serves to compare similar properties between non-buoyant and buoyant jets injected into a stationary ambient fluid.

The tendency for a buoyant jet to become self-similar means that the turbulent flow depends only on the buoyancy and weight deficit and that the overall properties obey the same rules over a wide range of scales (Zhou et. al. (2001)). Therefore, in the comparison to the available experimental data, the locations used in the analysis of the DREAM $^{\circledR}$ simulations are based on this observation.

For non-buoyant jets, the mean velocity development is in the axial direction, with the initial development region being between $0<\mathrm{x} / \mathrm{D}<15$, where $\mathrm{x}$ is the axial direction, which says that the profile changes from being approximately square to the normal circular shape within that period of time (Pope, 2000). For curved buoyant jets in

enclosures, the developmental region is not as clearly defined, as buoyancy forces tend to hinder or enhance the development of turbulences within the flow. Therefore, for the curved buoyant jet analysis, instead of using $x / D$, the coordinate direction of the jet development region, which in these simulations is approximately the vertical direction, is used. Thus, the development region is taken to be between $0<\mathrm{y} / \mathrm{D}<15$. The simulations presented in this work do in fact have certain wall effects present; however this fact doesn't prevent a comparison between the two cases. The reasons for this are that the jet will display similar properties as long as it is dominated mainly by the momentum flux. Also, the walls are sufficiently far from the inlet region to effect the development of the momentum driven part of the jet. It should be noted that only the 
inlet velocity of $0.25 \mathrm{~m} / \mathrm{sec}$ results are shown; however, the other inlet velocity flows exhibit similar self-similarity regions; however, because these locations are too close to the wall, the statistics begin to show wall effects.

In the buoyancy driven flows, the jet curves due to the density differences between the jet and the ambient fluid, thus for comparative purposes, the measurements must be normal to the jet itself. Pope (2000) defines the jets half-width $r_{1 / 2}$ to be the location where the jets velocity reaches exactly one-half of the centerline velocity. This definition is also used in the curved buoyant jet analysis.

Pope (2000) states that in non-buoyant jet flows, the jet decays and spreads with increasing axial distance, respectively. However, as the non-buoyant jet decays and spreads, the mean velocity profile changes, but the shape of the profile doesn't, and after $y / D=20$, with proper scaling, they collapse onto a single curve, indicating that the mean velocity profile is self-similar. Buoyant jets should display this property as well when the jet is predominantly momentum driven. The locations where the DREAM ${ }^{\circledR}$ simulations are examined are shown in Figure 7.2. These two locations have become predominantly buoyancy driven, and up to a certain time, wall effects are minimal on the behavior of the jet.

In the comparison between the non-buoyant and buoyant jet flows, the similarity variable is taken to be the jet distance divided by the half-width of the jet. The mean velocity profiles for the two buoyant jet locations, as compared to the Pope (2000) experimental data shows very good agreement in the self-similar regimes of the jet. This is evident in Figure 7.3.

Other important quantities must be determined to get a more accurate picture of the curved buoyant jet behavior within the similarity region. Pope (2000) shows that the Reynolds stresses become self-similar in the jet region. Hussein et. al. (1994) observed that on the centerline, the root mean square velocity is approximately one quarter of the mean in magnitude, and toward the edge of the jet, the ratio of the root mean square velocity fluctuations to the local mean velocity increases. A comparison between the available experimental data by Hussein et. al. (1994) to the DREAM ${ }^{\circledR}$ simulations within the similarity region is shown in Figure 7.4 as compared to the Hussein et. al. (1994) experiments. The DREAM ${ }^{\circledR}$ simulations appear to slightly under predict the magnitude 
of the stresses within the jet. The reason is most likely due to some influence of the wall on the jet, as well as the ever present implicit dissipation. Note that only the resolved stresses are depicted in Figure 7.3.

What is difficult to analyze and understand is the consequences of the turbulent kinetic energy within the jet, and the mechanisms that generate and dissipate turbulent kinetic energy. As no turbulent profile is specified at the inlet, all of the turbulence must come from the jet interactions with the surrounding fluid, i.e. initial jet inflow into stagnant environment and shear at the jet edge. The basic definitions of the Kolmogorov length and time scales allow some insight to be gained into both non-buoyant and curved buoyant jet flows. It is clear that the Reynolds number based on the Kolmogorov length scale is unity, indicating that the motions on these scales are viscosity driven. Therefore, the smallest motions decrease in size and timescale as the Reynolds number, or densimetric Froude number, increases. This indicates a fundamental difference in the energy budget between the non-buoyant and buoyancy driven jets, being that buoyancy tends to hinder turbulence (Pope, 2000), but in our case, buoyancy in fact enhances turbulence within the enclosure.

Due to the different mechanisms that govern the energy budget over the jet, i.e. dissipation, production, convection, and transport, a comparison of the scales present within the flow of non-buoyant and buoyant jets may be misleading. Throughout the non-buoyant jet flow, dissipation is the dominant term in the energy balance equation and the production goes to zero near the jet edge. Pope (2000) states that it takes a long time to dissipate an amount of energy $\mathrm{k}$, which is similar in time to produce $\mathrm{k}$ for non-buoyant jet flows. These time scales are approximately equal to the time it takes a particle released from the centerline of the inlet to travel from the origin to a certain point downstream of the inlet. Thus, the turbulence is found to be long lived within the jet center line region and as the flow becomes less turbulent within the jet, the smallest local scales become larger in size. Therefore, the resolvable scales are much greater than the Kolmogorov length scales and less important in the governing of the non-buoyant jet. However, for curved buoyancy driven flows, this effect is in fact magnified, as buoyancy forces in stably stratified environment hinder the development of turbulences toward the stably stratified side of the jet. However, in the non-stably stratified side of the jet, 
buoyancy acts to accelerate the flow, and enhances the turbulence within that region. Therefore, the smallest scales within the curved buoyant jet should be less important in the overall determination of the flow. This fact is supported by work performed by Zhou et. al. (2001), where a vertical buoyant jet was investigated using a Smagorinsky sub-grid scale model. In analysis of the Reynolds stresses, it was clear that the subgrid-scale stress contributed less to the total shear energy, indicating that the flow field is nearly fully resolved (Zhou et. al. (2001)). Thus, similar behavior may be deduced for the DREAM $^{\circledR}$ simulations, and an explicit sub-grid scale model may not be necessary for the analysis of curved buoyant jet flows.

The turbulent kinetic energy within the curved buoyant jet from the DREAM ${ }^{\circledR}$ simulations are presented in Figure 7.5. The turbulence is maximum near the jet centerline, and appears to decay outward. It is important to re-iterate that the wall effects have not been included in the analysis. However, it must be noted that across the mixing layer, the turbulence increases slightly and then decreases, which is because of the shear stress within the mixed layer. This shear is increases with increasing densimetric Froude number. Also, the axial component of the energy spectra is shown in Figure 7.6a, with the density fluctuations energy spectra within the similarity jet region shown in Figure 7.6b. Figure 7.6a exhibits the appropriate characteristics of the high-frequency inertial subrange, which again indicates an appropriate rate of energy transfer from the largest to the smallest resolved eddies. Again, the smallest resolvable eddies may be decreased in size with increasing grid resolution and decreasing time step. Figure 7.6b shows a similar trend in the inertial subrange, which is followed by a region that exhibits a decay of -3 power law. This is a special feature of some buoyant jet flows, whereby representing the inertial-diffusive subrange as suggested by List (1982). Because of this, it may be stated that buoyancy has a strong effect on the turbulence spectrum within the enclosure; being independent of whether or not turbulence generation is hindered or enforced. The jump between the $-5 / 3$ and -3 subrange may in fact be a result from the impact and energy feeding of impingement of the jet onto the wall. However, more study into this type of phenomena needs to be done. The energy spectrum observations from the velocity and density fluctuations further supports the use of an implicit turbulence model for curved 
buoyant jet simulations, as well as the accuracy of the simulations. These results are only valid up until the filling and the wall effects begin to effect the characteristics of the jet.

Figures $7.7-7.9$ show density field comparisons between the experiments and the DREAM $^{\circledR}$ simulations for the curved buoyant jet. In Figure 7.10 the centerline paths for the experiments and the fine grid simulations from DREAM ${ }^{\circledR}$ are presented. The densimetric Froude number dependence on the centerline trajectory is clearly visible. The fine grid simulations are able to accurately predict the centerline trajectories for all densimetric Froude numbers, which lends weight to the accuracy of the simulations. The error bars indicate maximum deviations from the mean centerline profile for the experiments, and the simulations fall within the error bars. One of the difficulties in comparing CFD simulations to experiments comes in the determination of the mixed layer thickness, especially for immiscible liquid-liquid flows. For the experiments, a three-dimensional picture is used, and since the data is taken from outside of the enclosure, it is difficult to compare to the centerline predictions from a two-dimensional planar view. Comparison of the DREAM ${ }^{\circledR}$ simulations, with the inclusion of the mixing layer assuming real mixing and not numerical diffusion, show that the results correlate well with the experimental data, although for the highest densimetric Froude number simulations, some deviations appear. Clearly, the amount of mixing predicted by the simulations is less than that collected through the experiments. This is most likely due to the time step used in the simulations. Since the projection method is used in all of the simulations, too large of a time step causes an increased amount of dissipation predicted and filters out too much of the small scale turbulence, which causes the shear at the edge of the oil/water layer. Thus, decreasing the time step will only enhance the codes ability to predict the features of the curved buoyant jet at high Reynolds numbers. Another difficulty in the simulations is that to accurately model a high Reynolds number and Froude number jet, some entrainment mechanism needs to be incorporated into the simulations to allow for an appropriate amount of shear and mixing to take place at the interface. The results of the high Froude number simulations clearly support this hypothesis, as the mixing and shear around the jet/ambient interface does not appropriately resolve the nature of the jet. However, this is not as important for the lower Reynolds number and Froude number simulations, whereby the mixed layer thickness is 
more accurately captured. Thus, for any entrainment mechanism to be accurately modeled, both Reynolds and Froude number dependence is needed, as well as accounting for the stably stratified interface.

However, much deeper insight may be gained from analyzing the DREAM ${ }^{\circledR}$ simulations for the jet behavior. With respect to the inner density boundary of pure fluid, it is clear that as the densimetric Froude number increases, the spreading of the jet inner boundary decreases, especially after the development region. This indicates that Reynolds number effects may be less subtle than what was initially thought. Clearly, the closer the density difference, the smaller in size the inner boundary is, as a larger density difference causes a widening of the inner jet widths. On the other hand, spreading of the outer boundary, where shear and mixing dominate the flow, increases with increasing densimetric Froude and Reynolds numbers. Thus, it may be concluded that the buoyancy effects in the outer boundary will dissipate, with momentum and shear forces governing the development with increasing densimetric Froude and Reynolds numbers. This fact only supports the conclusion for a need of some entrainment mechanism to model the physics at the boundary. Simulations show that the buoyancy effects on the inner jet region dominate as the densimetric Froude and Reynolds numbers are decreased.

Thus, in conclusion, in evaluation of curved buoyant jets, the momentum driven region exhibits similarity, which is identical in behavior to non-buoyant jet flows. The turbulent kinetic energy is maximum near the jet centerline within this inner region for both buoyant and non-buoyant flows. The buoyancy forces present within the flow both hinders and enhances the turbulent kinetic energy, which reduces the effects of the small scale structures away from the centerline toward a stably stratified interface, but enhances the turbulence near the non-stably stratified locations. This in turn indicates that for small Reynolds and densimetric Froude numbers, the inner jet region is larger when compared to simulations at higher dimensionless parameters. The opposite behavior is expected for the outer jet region.

\subsubsection{Global Effects of the Buoyant Jet}

Buoyancy effects arise in a group of problems that include atmospheric and oceanic boundary layers, refueling processes and discharge. Apart from generating 
turbulence anisotropy, buoyancy gives rise to changes of turbulence structure and intensity in much the same way as streamline curvature does, allowing either enhancement or suppression of turbulence. Unfortunately, the behavior of buoyancy driven jets impinging on density interfaces or wall is not a well understood phenomenon. For the flows described in this study, the fuel is injected at the center of the domain, which means that as the fuel enters the domain, buoyancy forces cause the jet to become curved and rise upwards. Thus, as the density increases in the direction of gravity, which is downward in the vertical direction, is stably stratified, and thus the turbulent energy is suppressed. The reason is that in general, for the turbulent energy to be generated, the shear production must exceed that of the buoyancy suppression to survive, and also, stabilization alters the Reynolds shear stress (Durbin and Reif (2001)).

The buoyant forces cause variations in the density field due to the differences in the scalar field concentration, giving rise to a fluctuating body force in the vertical momentum direction, effecting both the mean flow field and the turbulence present within the enclosure. In general, experiments have found that turbulence can't be sustained under the stabilizing effect if the local densimetric Froude number is less than 0.5. This local densimetric Froude number is an inner Froude number, where density differences are not included. This fact explains some of the behavior that is viewed in the analysis of the jet behavior, as well as in the effects on the overall domain after wall impingement.

Figures $7.10-7.12$ show instantaneous velocity magnitude contours for the three different densimetric Froude numbers for the fine grid simulations. Clearly, after the impaction of the jet onto the upper boundary, the energy loss and transfer of the curved buoyant jet results in a production of turbulence, which is transferred to the flow field, resulting in smaller vortical structures. Figures $7.13-7.15$ show the instantaneous contour plots of the normalized vorticity magnitude after impaction. There is clearly more structures within the flow field after jet impaction, indicating the generation of turbulence throughout the effected regions. This type of phenomenon increases with densimetric Froude number and is less visible for under-resolved flow fields, such as the coarser grid simulations, which utilize a 180x120x60 grid size. The instantaneous velocity vectors are shown in Figures 7.16 - 7.21, with Figures 7.16, 18, and 20 being 
colored by their span wise velocity component, which are shown at dimensionless times after jet impaction. These figures clearly show not only the planar direction of the flows, but also the three-dimensional turbulence effects throughout the flow field. Figures 7.17, 19, and 21 are at dimensionless times just after the curved buoyant jet impact with the top boundary, which produces large vortices within the chamber. The instantaneous density contours for the three densimetric Froude numbers are shown in Figures 7.22 - 7.24, again showing the turbulent nature of the flow field.

To further examine the results of the curved buoyant jet after impaction on the global flow field within the enclosure, the averaged turbulent flow characteristics are examined for the coarse and fine grid simulations. However, only the Fr $=7.66$ data is presented. It is noted in advance that a similar trend is observed for the other densimetric Froude number simulations.

Figures $7.25 \mathrm{a}, \mathrm{b}$, and c show the average velocity axial, vertical, and spanwise profiles at $\mathrm{x}=0.15 \mathrm{~m}$ and $0.3 \mathrm{~m}$, downstream of the jet inlet. It is interesting to note that the curved jet path is clearly visible in Figure 7.25a, as the maximum velocity components are at the jet crossing locations. Small differences are present between the two grid simulations; however, the fine grid resolution clearly predicts the velocity profiles more accurately outside of the jet. One of the difficulties in performing LES simulations, especially with ITM methods, is the ability to achieve grid independent results. Analysis of the mean streamwise components in Figure 7.25 indicates that this is not achieved with the finest grid resolution. To test this, the 120x80x40 simulations were utilized, which gives axial velocity data for three different grids. Richardson extrapolation is performed extrapolate for a grid independent solution. Figure 7.26 shows these results, with error bars being plotted on the extrapolated solution. The error bars, for the most part, are within 5\% of the fine grid solution, which allows for the conclusion of an approximate grid independent solution for the fine grid simulations. However, it must be noted that with an increase in the grid resolution, the ability to resolved the fine scales within the flow field increases. Thus, to achieve the extrapolated solution, the grid size increase may be double of the current fine grid results. The axial behavior of the flow after jet impaction is examined, at $\mathrm{y}=0.2 \mathrm{~m}$ and $\mathrm{y}=0.4 \mathrm{~m}$, or in the jet center. The 
mean velocity profiles are shown in Figure 7.27 a, b, and c for the axial, vertical and spanwise components.

The mean velocity r.m.s. quantities are examined at the two axial and vertical locations within the enclosure for the two different grids in Figures 7.28 (a,b, and c) and 7.29 (a,b, and c). Again, the curved jet crossing is clearly visible, as well as the fact that after impaction, the velocity fluctuations increase in magnitude, which lends weight to the conclusion that there is a generation of smaller vortices throughout the flow. The square of the turbulence intensities, as shown in Figures 7.30 (a,b, and c) and 7.31 (a,b and c) show similar behavior, with the mean axial components being the largest for all simulations. This indicates that even at impaction, the jet still maintains considerable amounts of its initial momentum.

For comparative purposes, the resolved turbulent kinetic energies within the enclosure are examined for the two grid simulations, as well as for Fr $=7.65$ and 1.9, at the four locations in Figures 7.32 - 7.35. The influence of the jet impaction is clearly visible, with the kinetic energies going to zero below the jet inlet height. Generally, the impact location has a large influence on the amount of kinetic energy within the enclosure, as closer to the impact region results in higher amounts of turbulence.

The total energy spectra are examined in Figures 7.36 - 7.39. Again, as with the analysis within the similarity region, the higher frequency inertial subrange behavior is captured, with some of the lower frequency energy being capture. This indicates that again, the largest vortical structures are at or near the jet/jet interface regions and an appropriate amount of energy is cascaded to the smaller vortical structures at an appropriate rate, particularly after jet impaction. However, with the other simulations presented in this work, the amount of dissipation seen in the simulations may be decreases through decreasing of the time step used in the simulations.

Therefore, the application of LES and ITM methods to variable density, curved buoyant jet flows shows that there is a generation of turbulence within the flow after impact onto the solid wall, a fact seen even with the amount of dissipation predicted in the simulations with a large time step. The effects of buoyancy both enhance and damp the turbulence presented in the flow, which is dependent on the stratification of the liquid-liquid interface. The location of this impact clearly affects the turbulent flow field 
quantities. Resolution of the flow field allows for the prediction of the turbulence, with the high frequency inertial subrange being resolved to an acceptable level. As with all ITM methods, the simulations within this section also show the difficulty in predicting the low frequency turbulence. These facts lend weight to the conclusion that DREAM ${ }^{\circledR}$ is capable of solving for curved buoyant jet flows, and appropriately predicting those quantities with a high degree of success using appropriate grid resolution. Decreasing of the time step in the simulation will reduce the amount of dissipation seen in the Quasisecond order upwind scheme utilized in the scalar solver, as well as the velocity components, which utilize a power-law scheme in the predictor-corrector method utilized in DREAM ${ }^{\circledR}$. This was evident in Chapter 6.0; however, resource limitation forced the use of the parameters as described in this section.

It is also useful to compare the behavior of the vertical buoyant jet to the curved buoyant jet simulations with comparable dimensionless parameters and inlet velocity distribution. Unfortunately, as indicated in Chapter 6, timestep and grid resolution are crucial, and because of the lack of computational resources, similar time-steps and resolutions are not available for the curved buoyant jet domain. However, some useful information may still be gained from a comparison. As shown in Chapter 7, the turbulent kinetic energy within the curved buoyant jet region, $\mathrm{U}_{\mathrm{in}}=0.25 \mathrm{~m} / \mathrm{sec}$ and a densimetric Froude number equaling 1.91 before wall impact, is difficult to quantify based on the experiment. However, utilizing the vertical buoyant jet simulations, $\mathrm{U}_{\mathrm{in}}=0.25 \mathrm{~m} / \mathrm{sec}$ and a densimetric Froude number equaling 2.8, in Chapter 6, a rough comparison may be made. Figure 8.1 shows a comparison between the curved buoyant jet and vertical buoyant jet simulations from DREAM. Clearly, the resolved turbulent kinetic energy of the curved buoyant jet is larger near the jet center, and this may indicate that the acceleration of the jet due to density stratification is not the leading cause of the increased amount of turbulence, as this is present within the vertical buoyant jet as well. Thus, although great care was taken to ensure that wall effects were not included in the analysis, both the impact of the jet on the upper boundary and the near wall behavior of the jet after it enters the tank indicates that wall effects within the curved buoyant jet domain can't be easily eliminated. Figure 8.1 confirms the expectation that under similar conditions, the curved buoyant jet seems to be more unstable. 


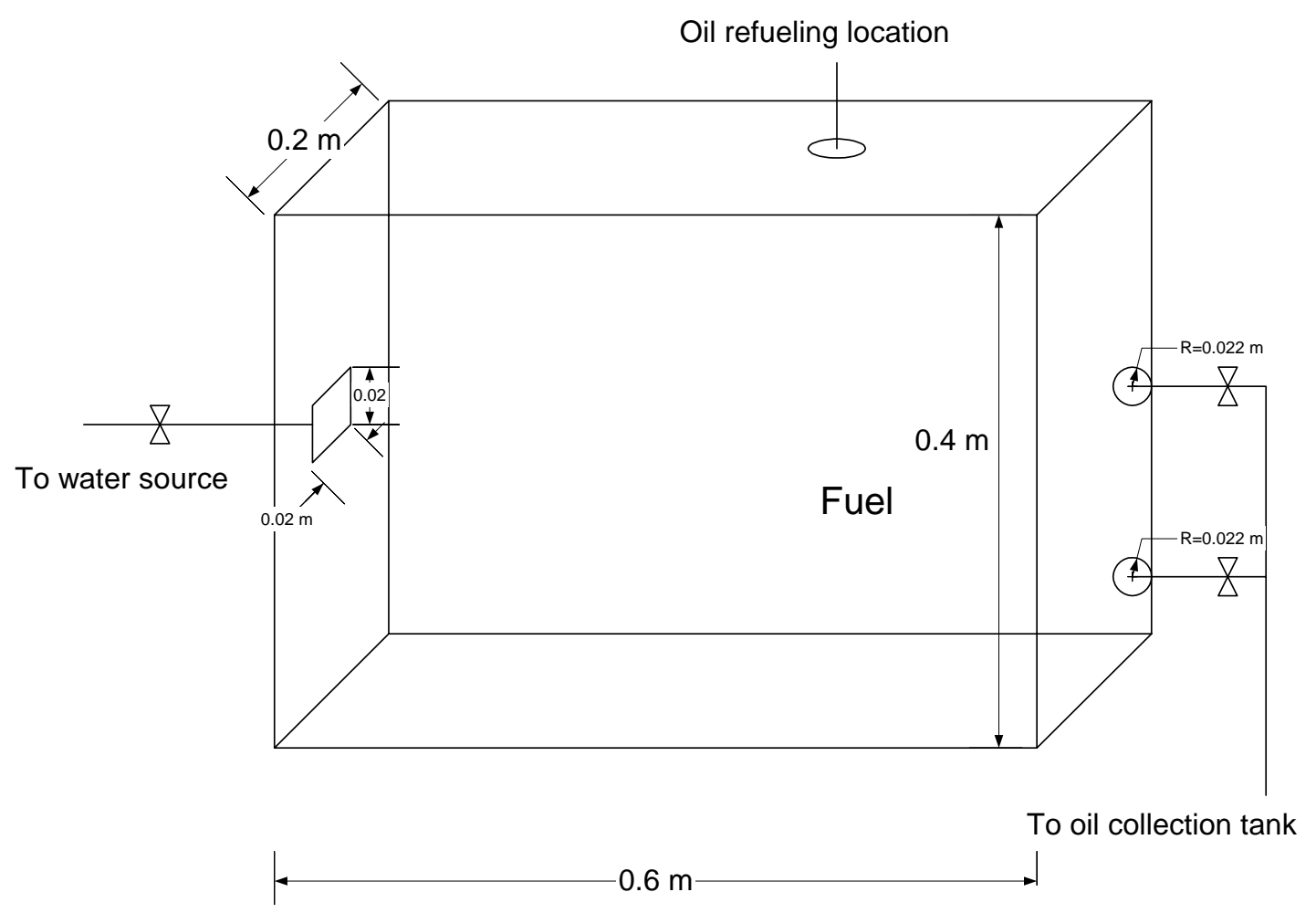

Figure 7.1 - Experimental setup used in the validation of DREAM ${ }^{\circledR}$ code 


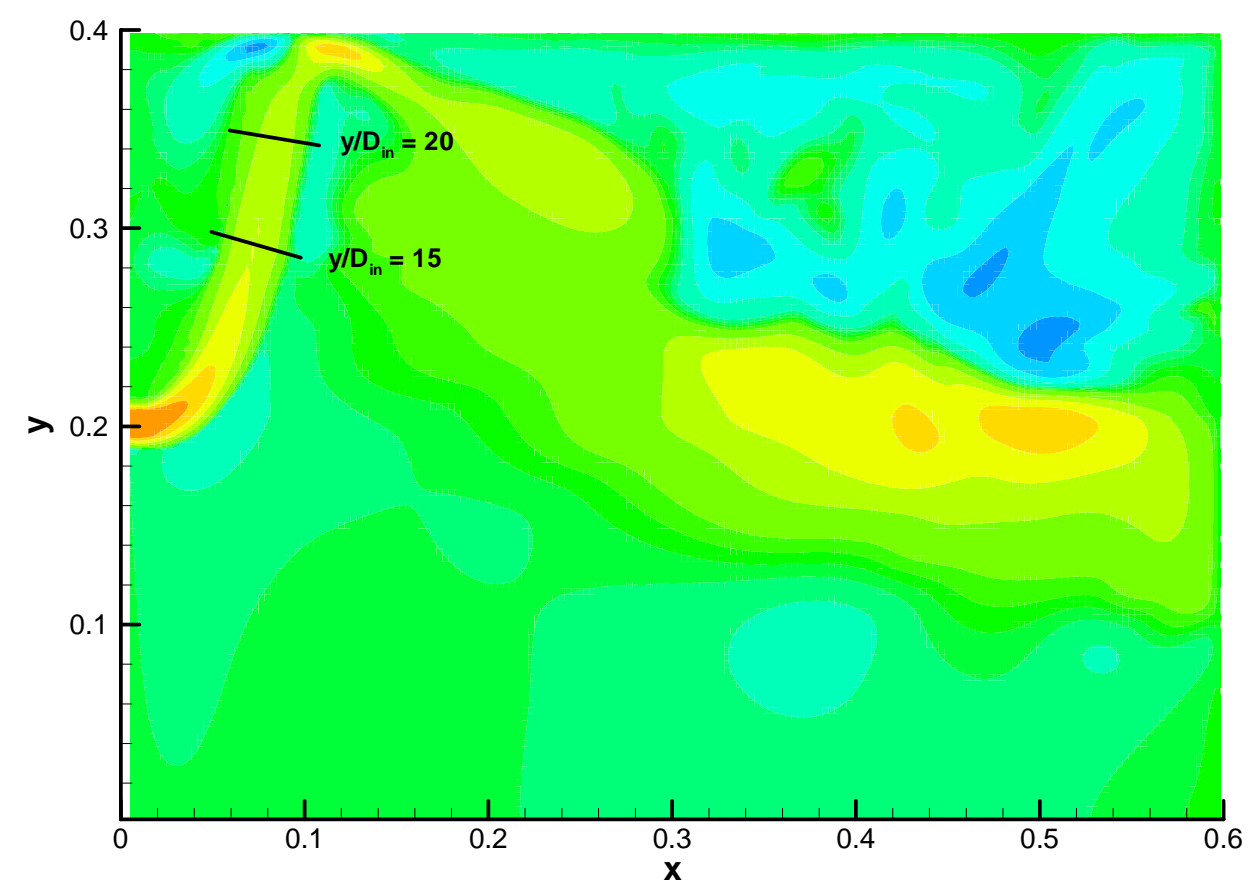

Figure 7.2 - Locations where jet data was obtained for similarity analysis

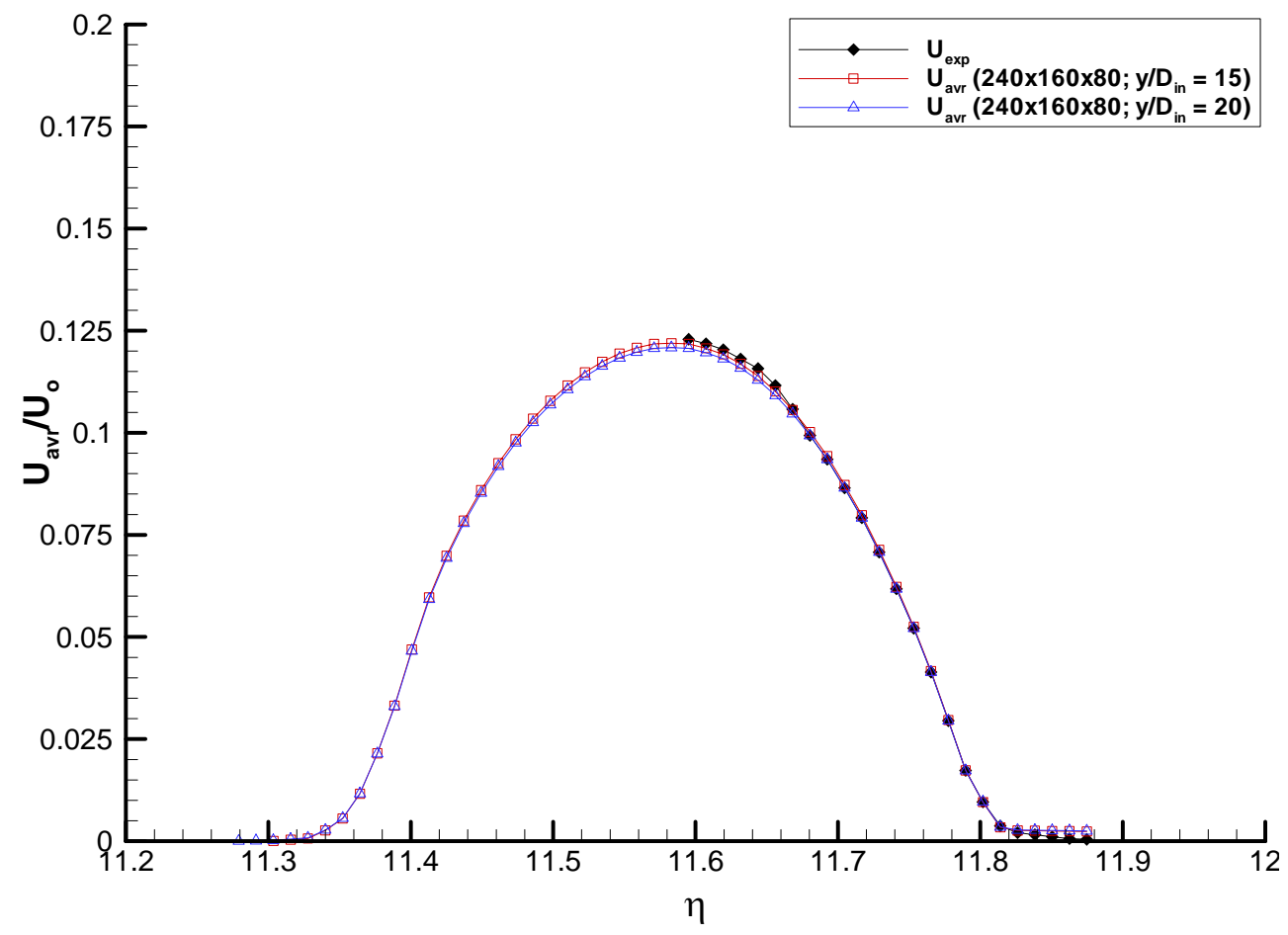

Figure 7.3 - Mean velocity profile within jet region at $y / D_{\text {in }}=15.0$ and 20.0 within the similarity regions of jet compared to experimental data of Hussein et. al. (1994) (from Pope, 2000). $U_{0}$ is the inlet velocity. 


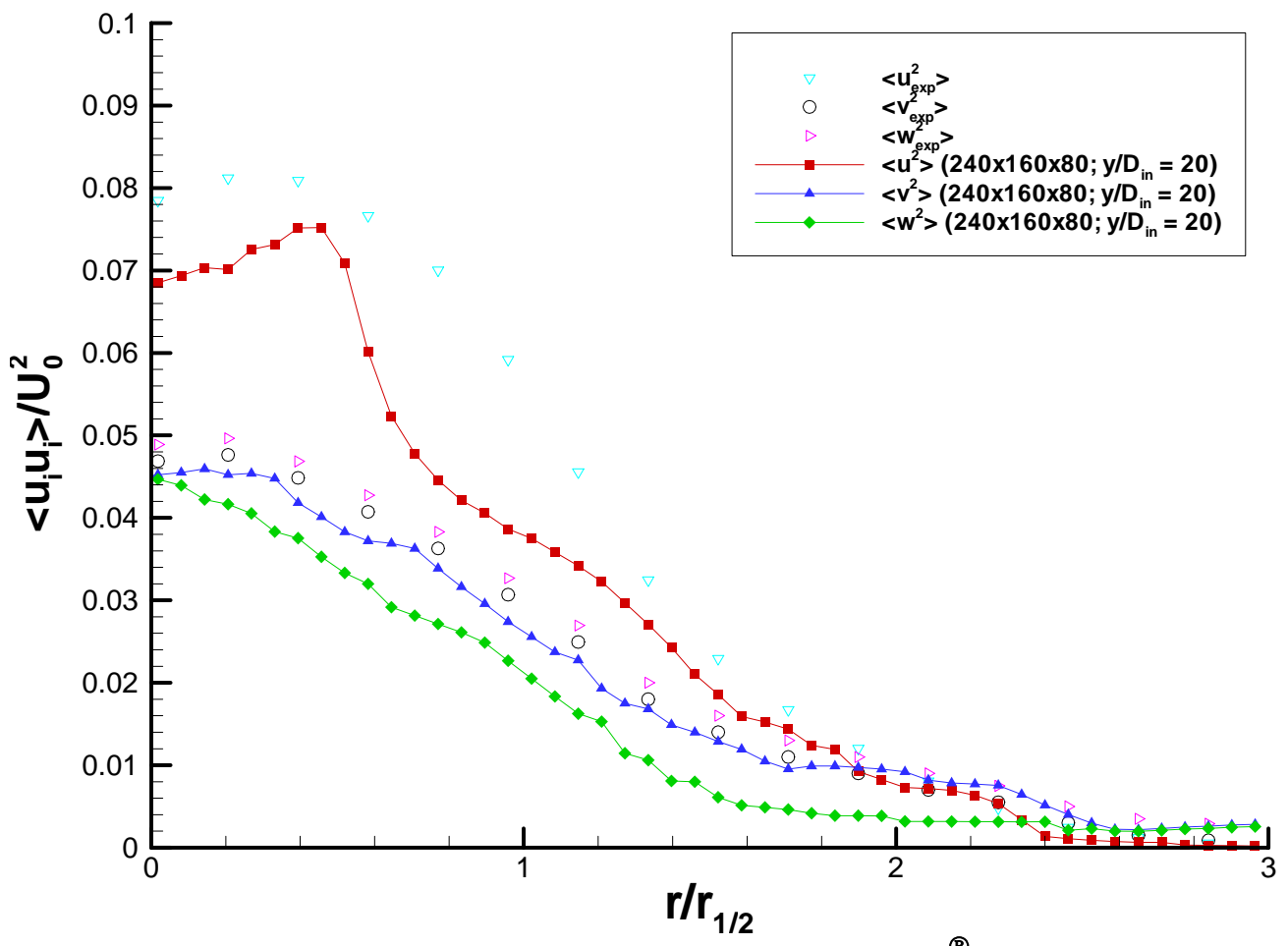

Figure 7.4 - Turbulence intensity squared from DREAM ${ }^{\circledR}$ simulations within jet region at $\mathrm{x} / \mathrm{Din}=20$ for $240 \times 160 \times 80$ grid compared to experimental data from Hussein et. al. (1994).

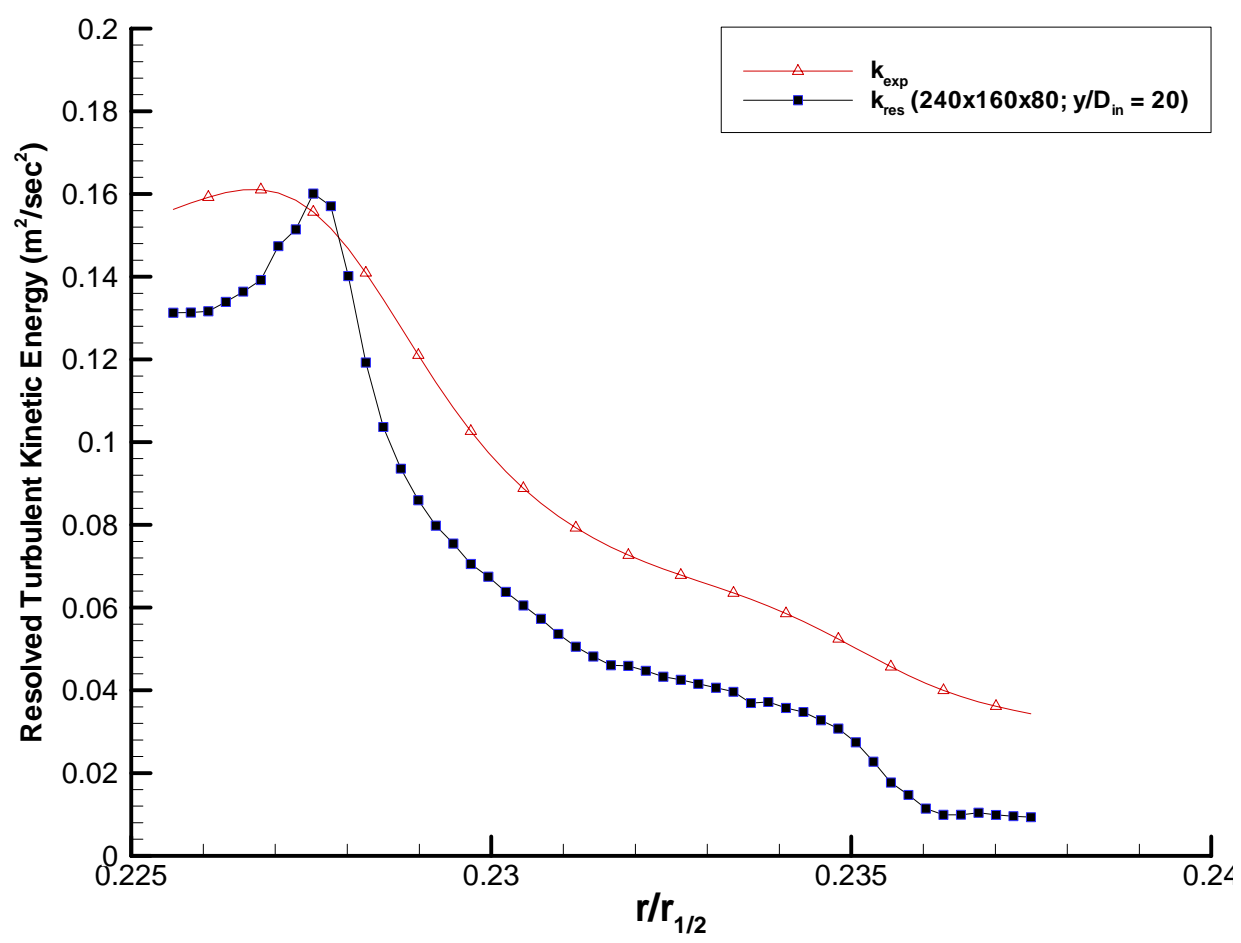

Figure 7.5 - Normalized resolved turbulent kinetic energy in half jet within the selfsimilar region of $y / D_{i n}=20$ for $240 \times 160 \times 80$ grid; $d t=0.001$ compared to experimental data from Hussein et. al. (1994). 


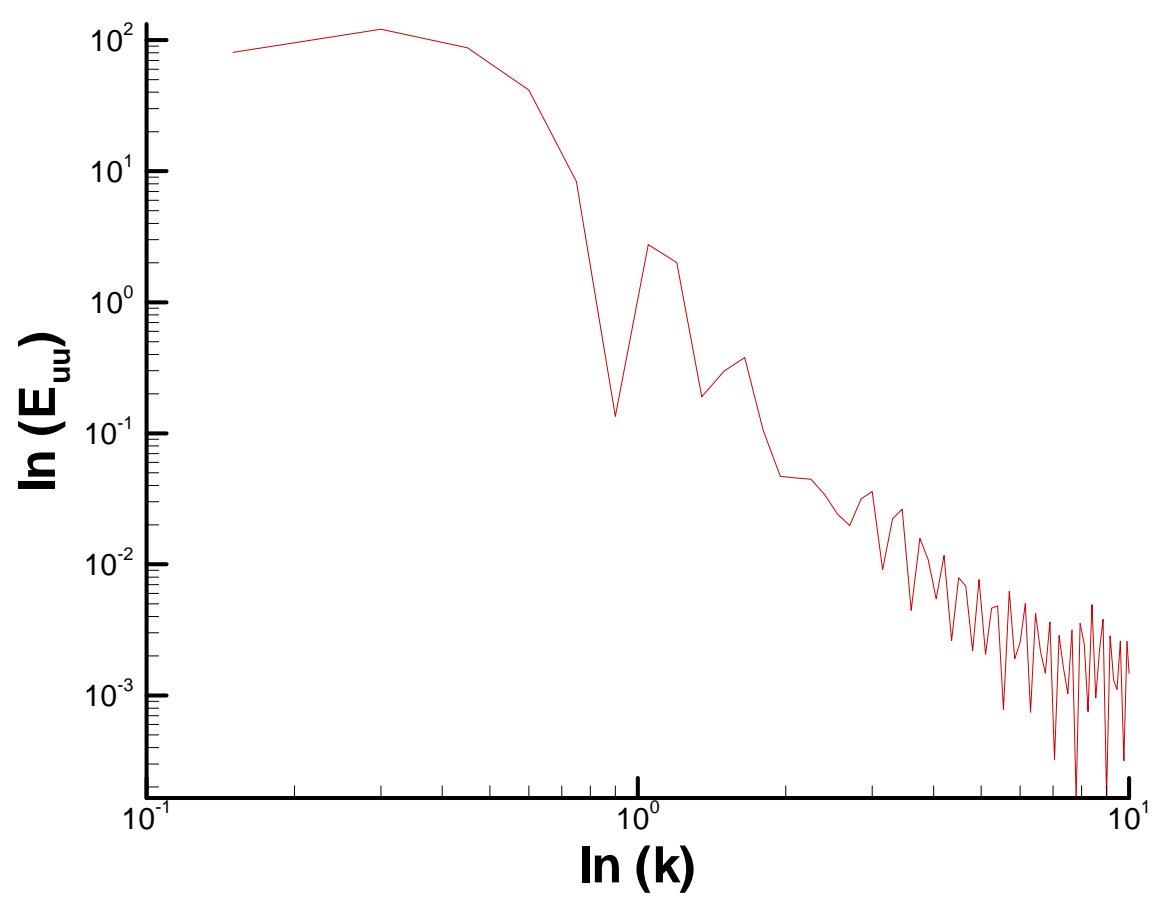

(a)

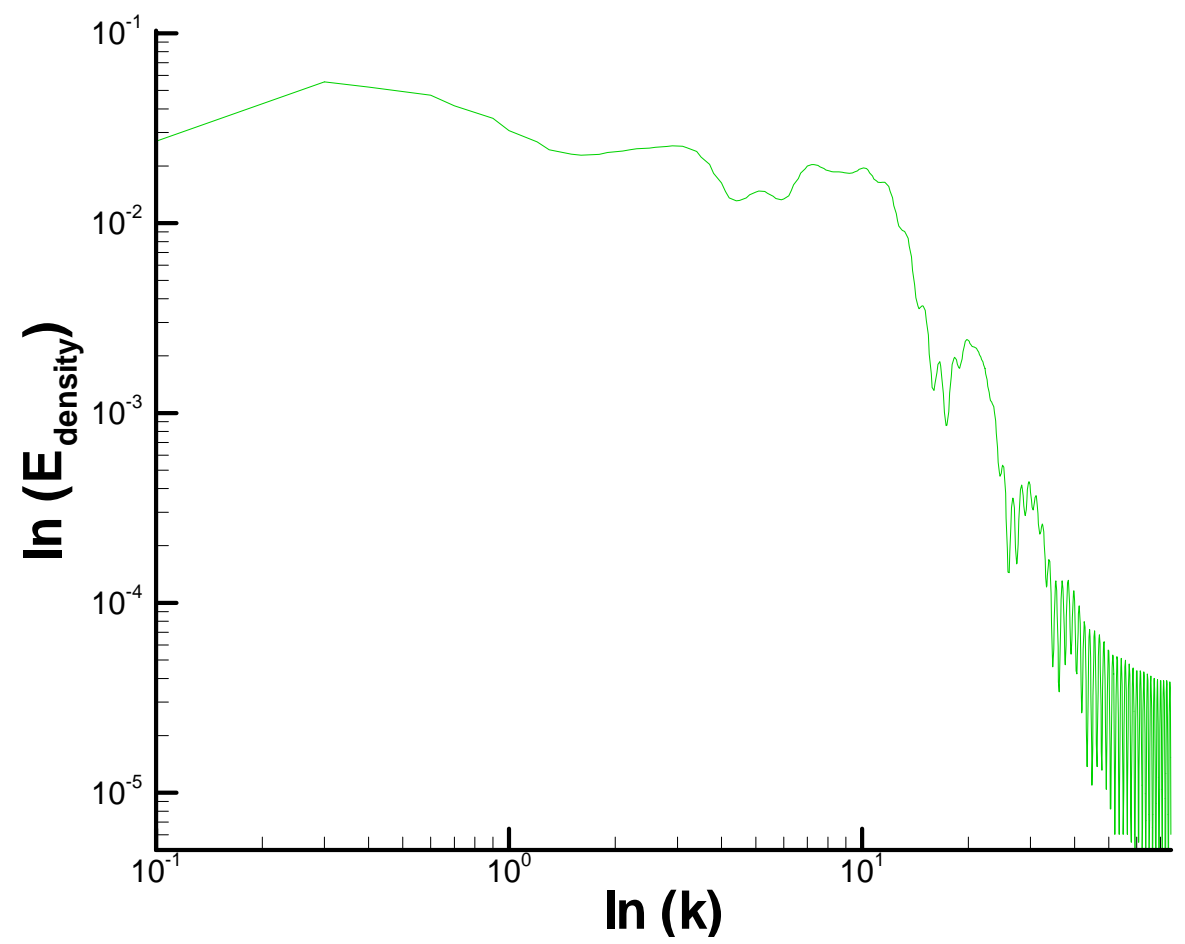

(b)

Figure 7.6 - Energy spectra for (a) the streamwise component and (b) density fluctuations in half jet within the similitude region of $y / D_{i n}=20$ for $240 \times 160 \times 80$ grid; $\mathbf{d t}=\mathbf{0 . 0 0 1}$ sec. 


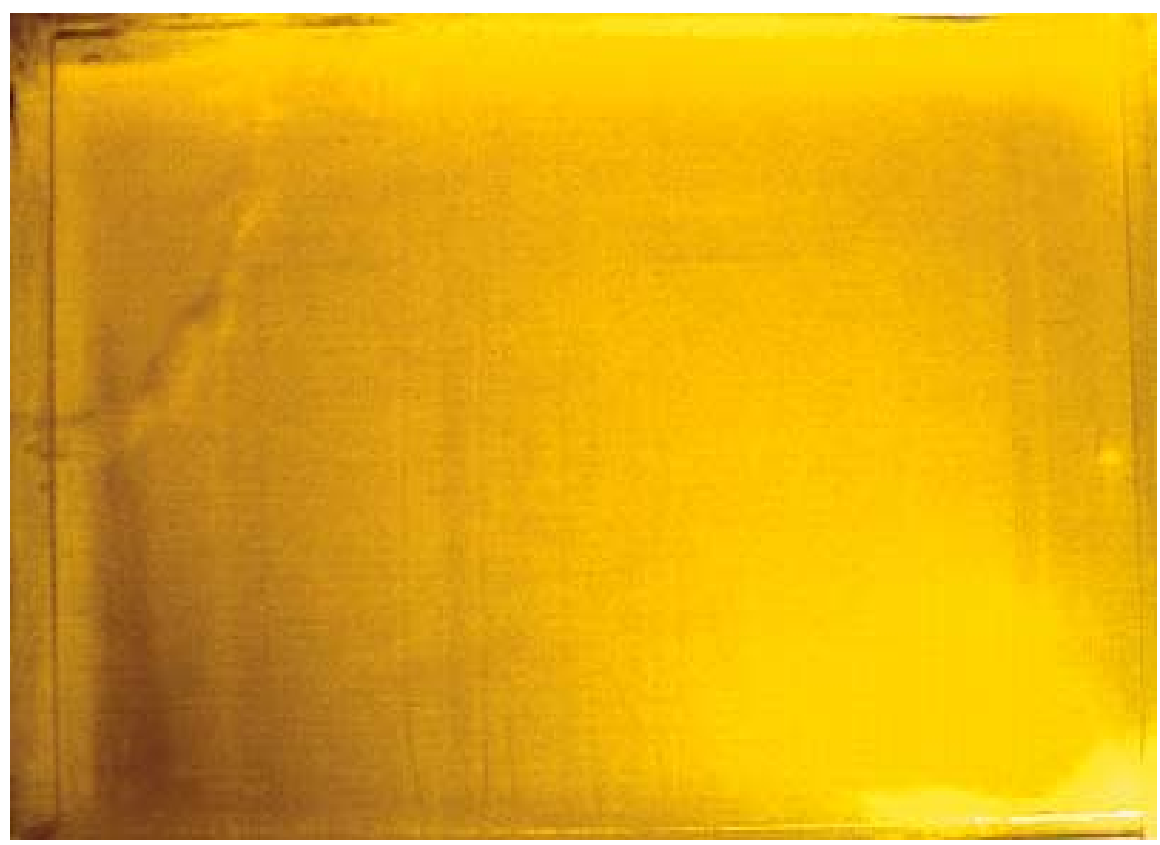

(a)

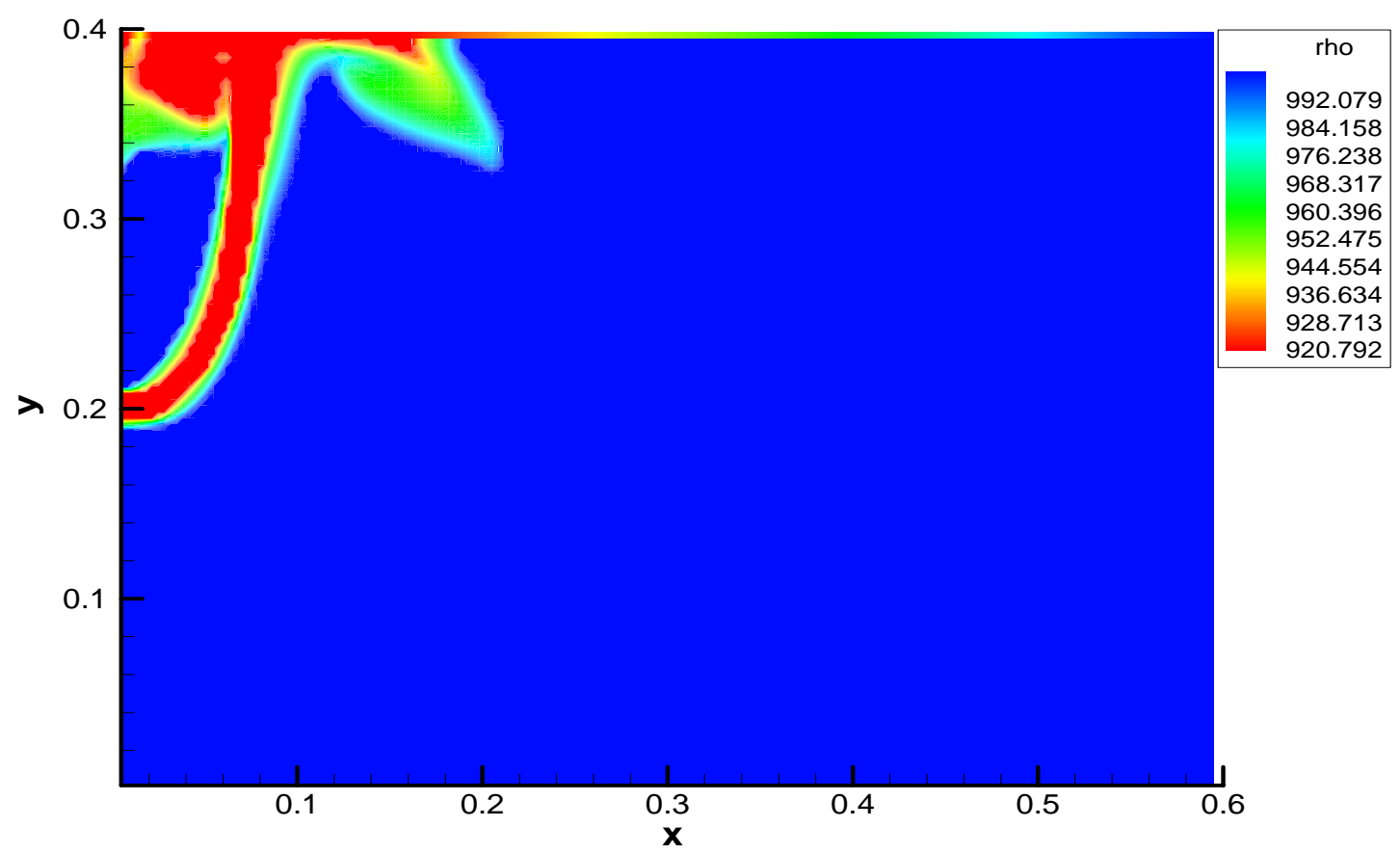

(b)

Figure 7.7 - (a) Experimental picture of inverted curved buoyant jet for $U_{\text {inlet }}=0.25$ $\mathrm{m} / \mathrm{sec} ; \mathrm{t}=18.0$ seconds, (b) DREAM ${ }^{\circledR}$ density contour for $240 \times 160 \times 80$ grid for $U_{\text {inlet }}$ $=0.25 \mathrm{~m} /$ sec. $; \mathrm{dt}=0.001 ; \mathrm{t}=18.0$ seconds; $\mathrm{Fr}=1.91$. 


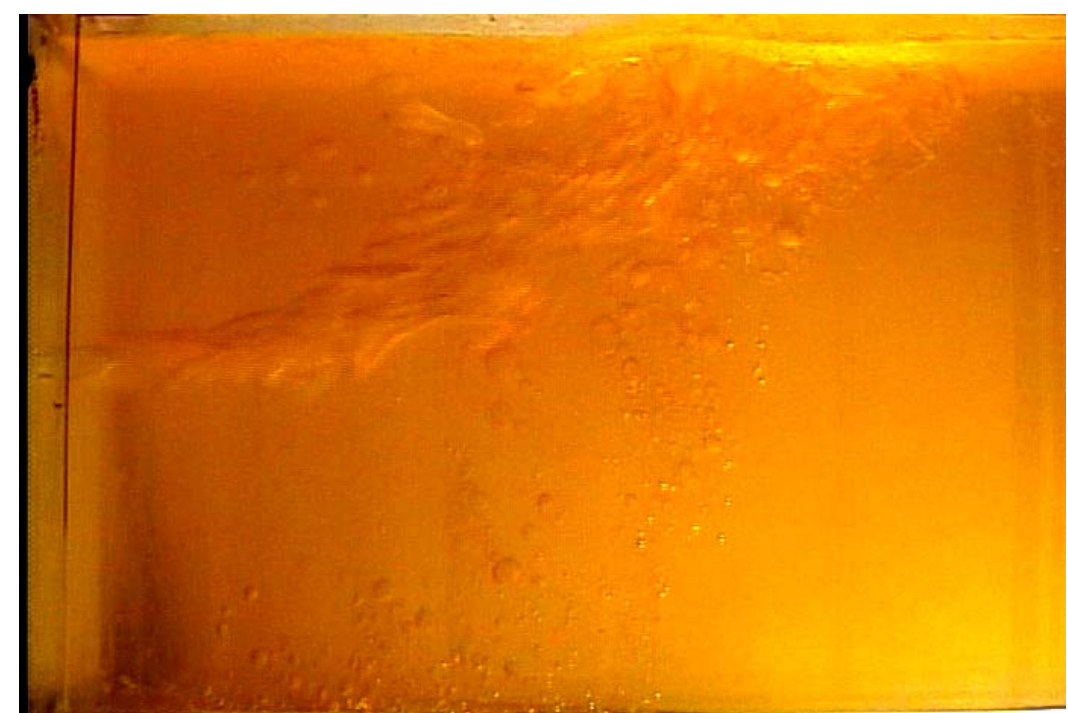

(a)

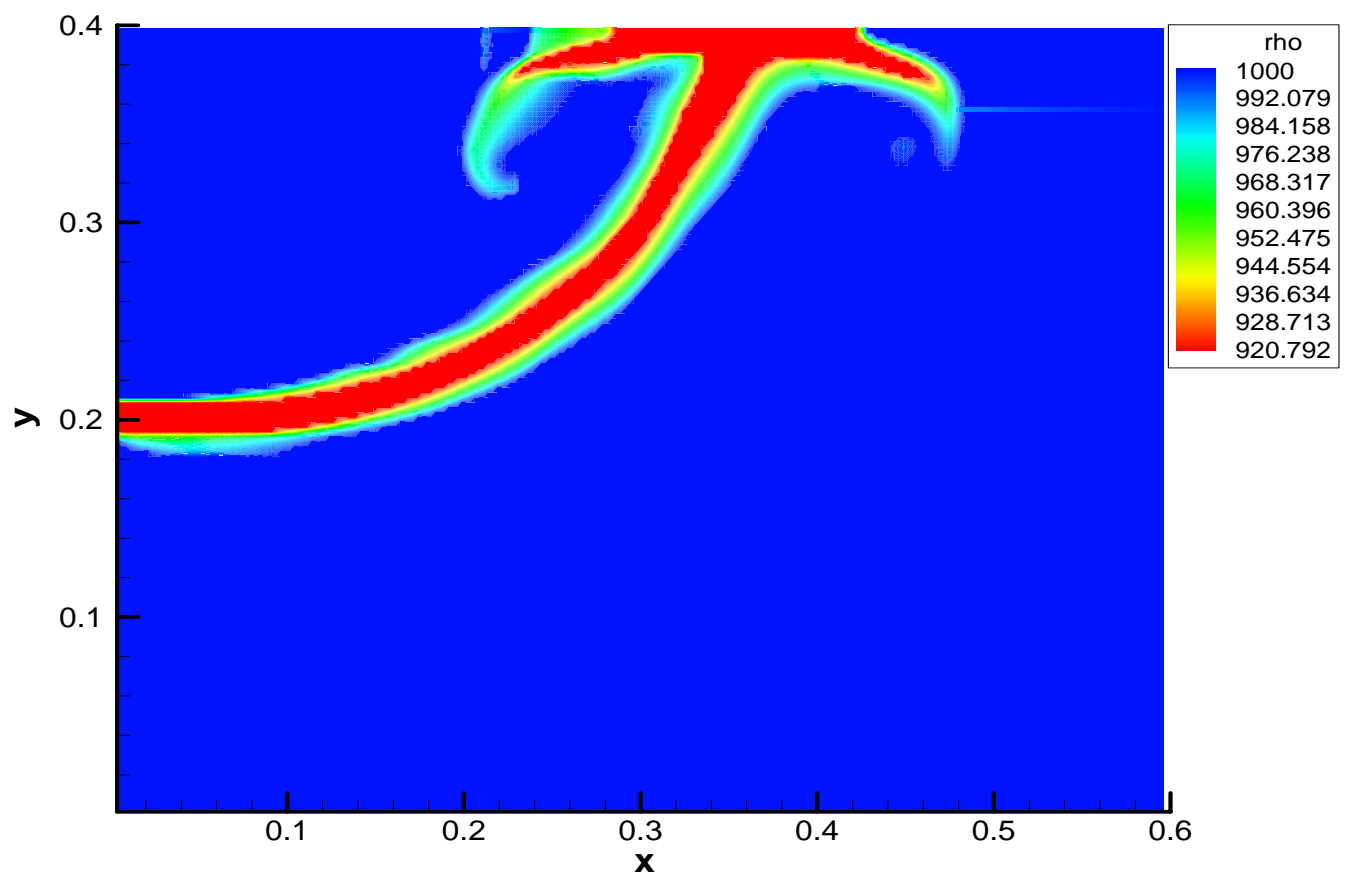

(b)

Figure 7.8 - a) Experimental picture of curved buoyant jet for $U_{\text {inlet }}=1.0 \mathrm{~m} / \mathrm{sec}$ (inverted for comparative purposes). b) DREAM ${ }^{\circledR}$ density centerline contour for $240 \times 160 \times 80$ grid for $U_{\text {inlet }}=1.0 \mathrm{~m} / \mathrm{sec}$.; $\mathrm{dt}=0.001 ; \mathrm{t}=5.0$ seconds; $\mathrm{Fr}=7.97$. 


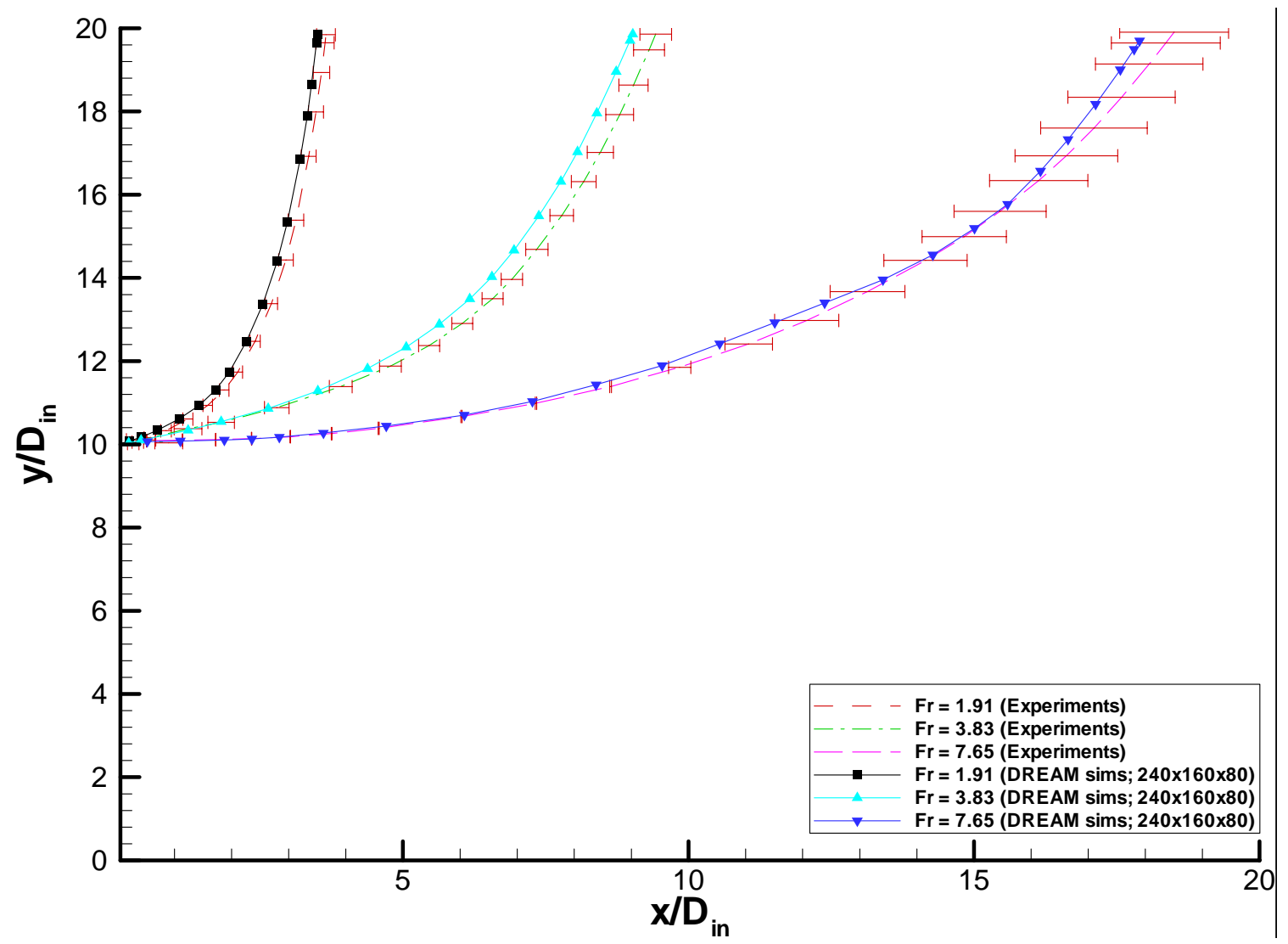

Figure 7.9 - Comparison of densimetric Froude number dependence for curved buoyant jet centerline path between experiments and DREAM ${ }^{\circledR}$ simulations for 240x160x80 grids; dt $=0.001$ sec. Error bars represent maximum variations between the sixteen different experiments. 


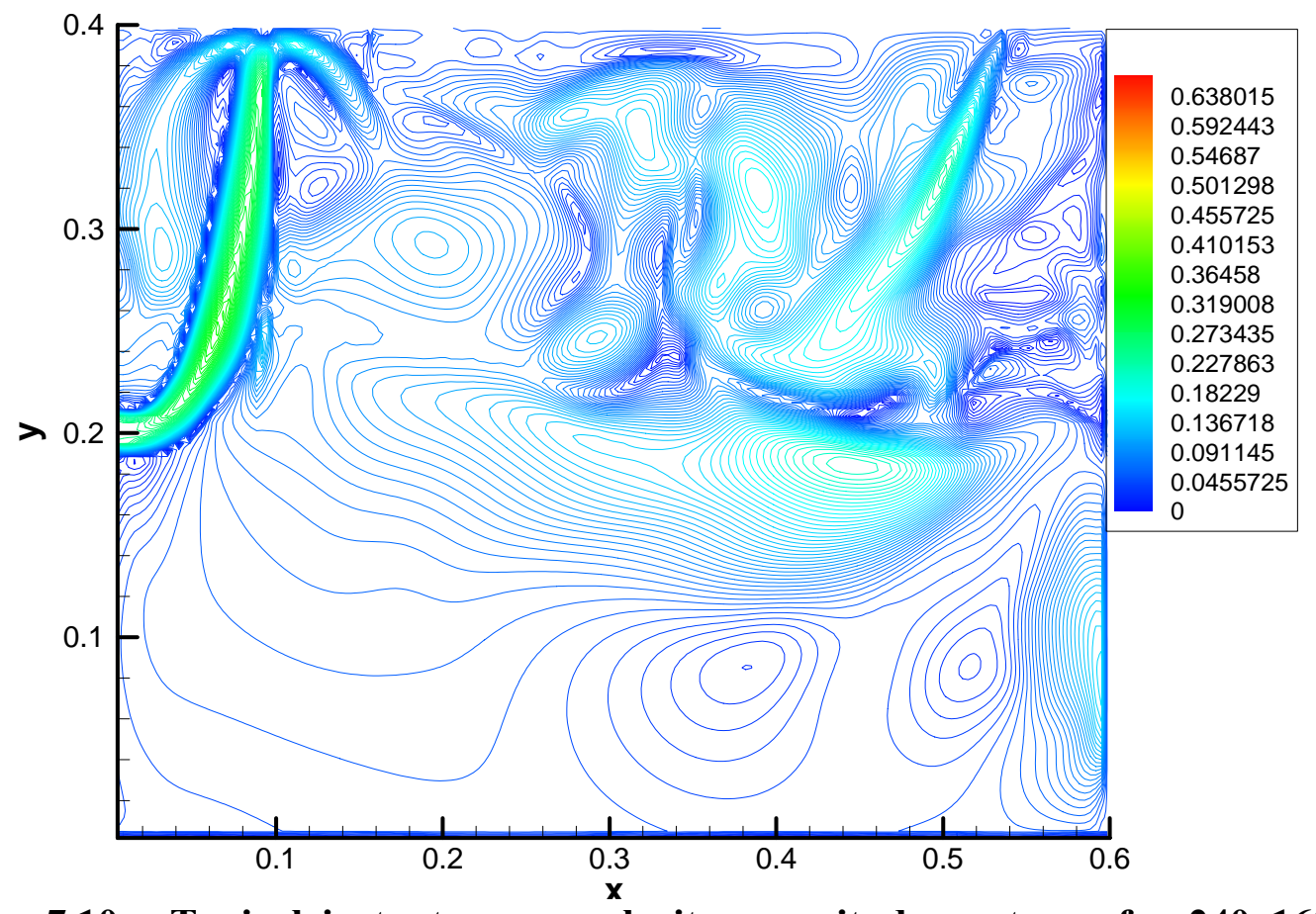

Figure 7.10 - Typical instantaneous velocity magnitude contours for $240 \times 160 \times 80$ grid; $d t=0.001 ; U_{\text {inlet }}=0.25 \mathrm{~m} / \mathrm{sec} ; \mathrm{t}^{*}=0.1875 ; \mathrm{Fr}=1.91$

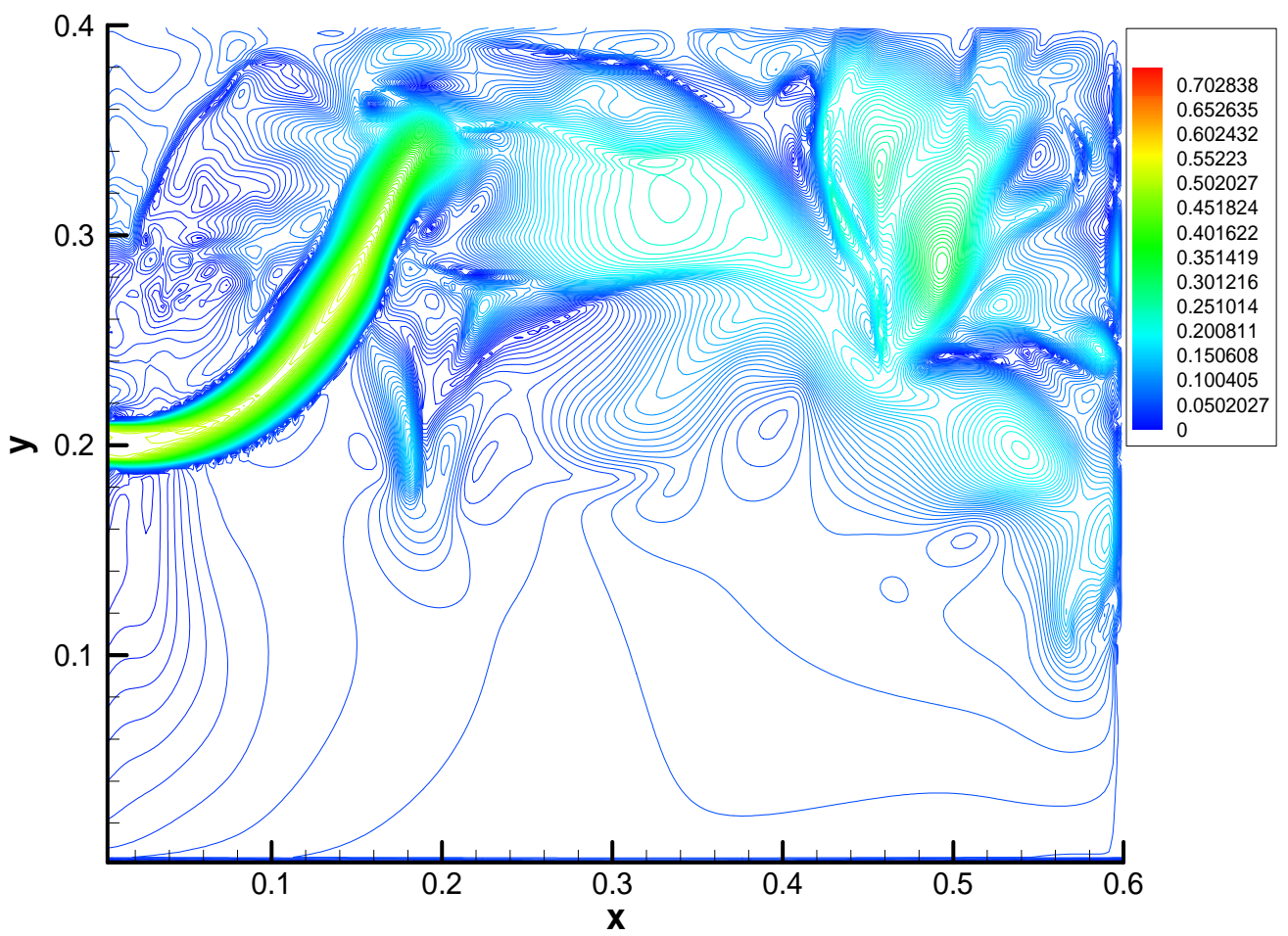

Figure 7.11 - Typical instantaneous axial velocity contours for $240 \times 160 \times 80$ grid; $d t=$ $0.001 ; U_{\text {inlet }}=0.5 \mathrm{~m} / \mathrm{sec} ; \mathrm{t}^{*}=0.25 ; \mathrm{Fr}=3.83$ 


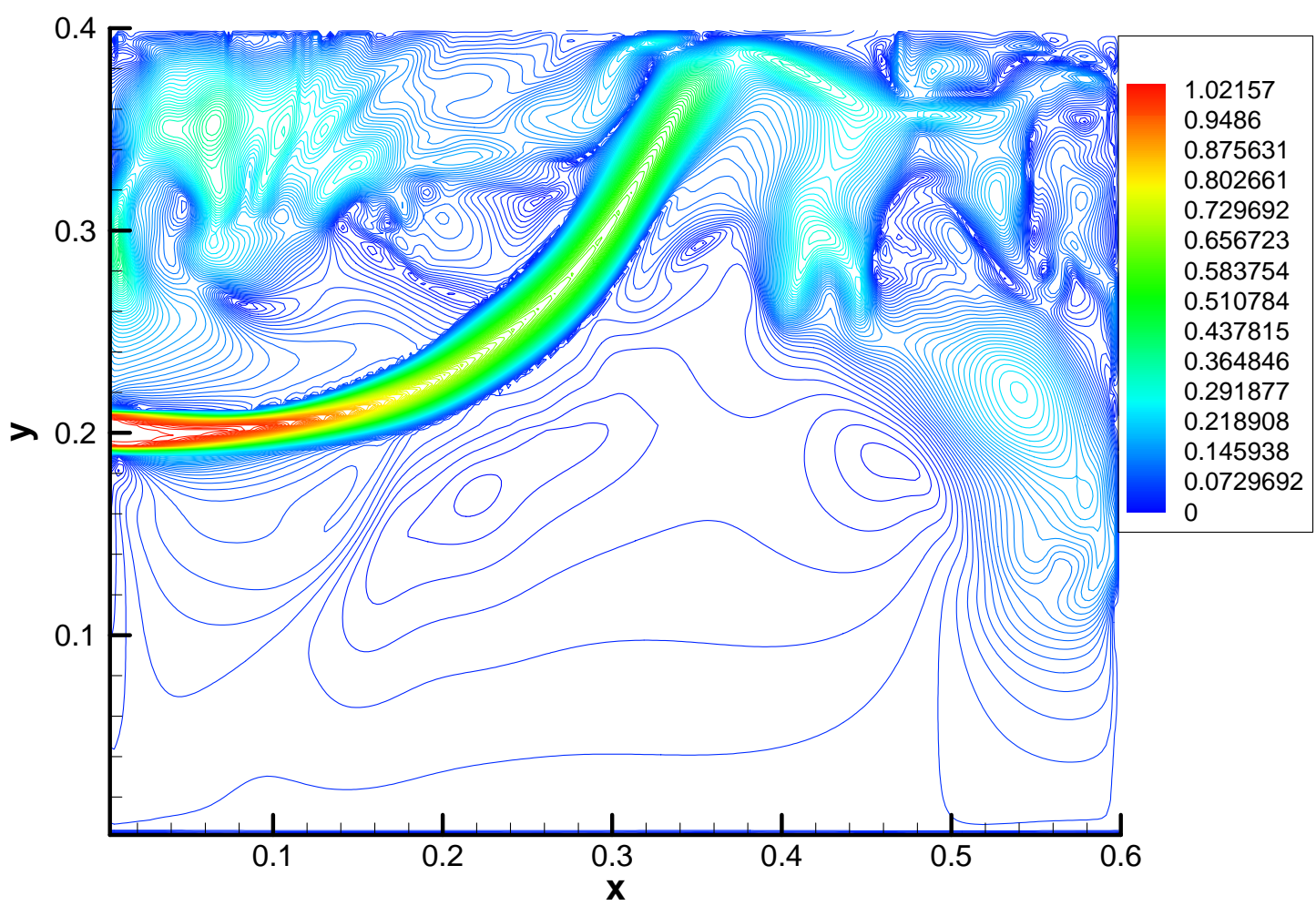

Figure 7.12 - Typical instantaneous velocity magnitude contours for $240 \times 160 \times 80$ grid; $\mathrm{dt}=0.001 ; \mathrm{U}_{\text {inlet }}=1.0 \mathrm{~m} / \mathrm{sec} ; \mathrm{t}^{*}=0.15 ; \mathrm{Fr}=7.65$

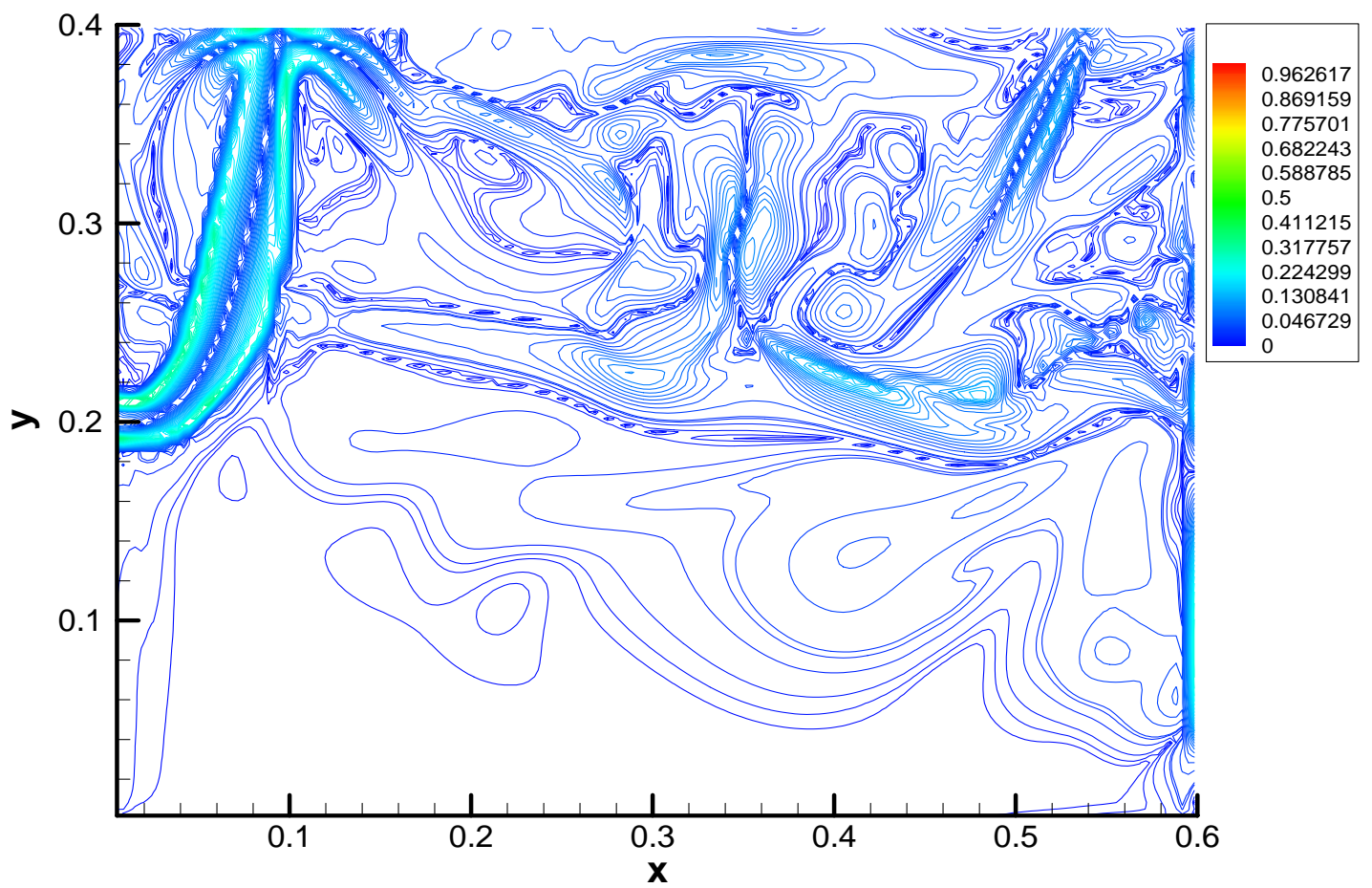

Figure 7.13 - Typical instantaneous vorticity magnitude contours for $240 \times 160 \times 80$ grid; $\mathrm{dt}=0.001 ; \mathrm{U}_{\text {inlet }}=0.25 \mathrm{~m} / \mathrm{sec} ; \mathrm{t}^{*}=0.1875 ; \mathrm{Fr}=1.91$ 


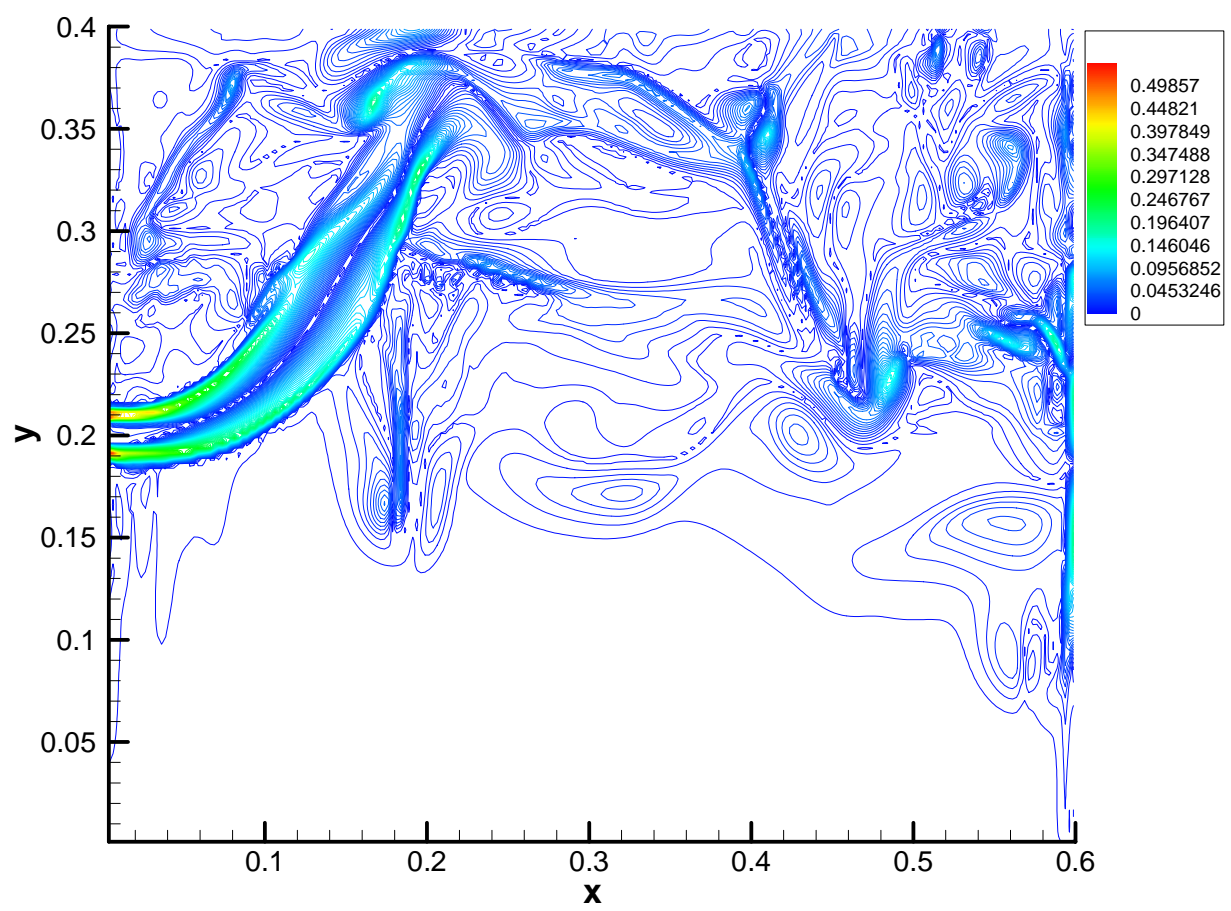

Figure 7.14 - Typical instantaneous vorticity magnitude contours for $240 \times 160 \times 80$ grid; $\mathrm{dt}=0.001 ; \mathrm{U}_{\text {inlet }}=0.5 \mathrm{~m} / \mathrm{sec} ; \mathrm{t}^{*}=0.25 ; \mathrm{Fr}=3.83$

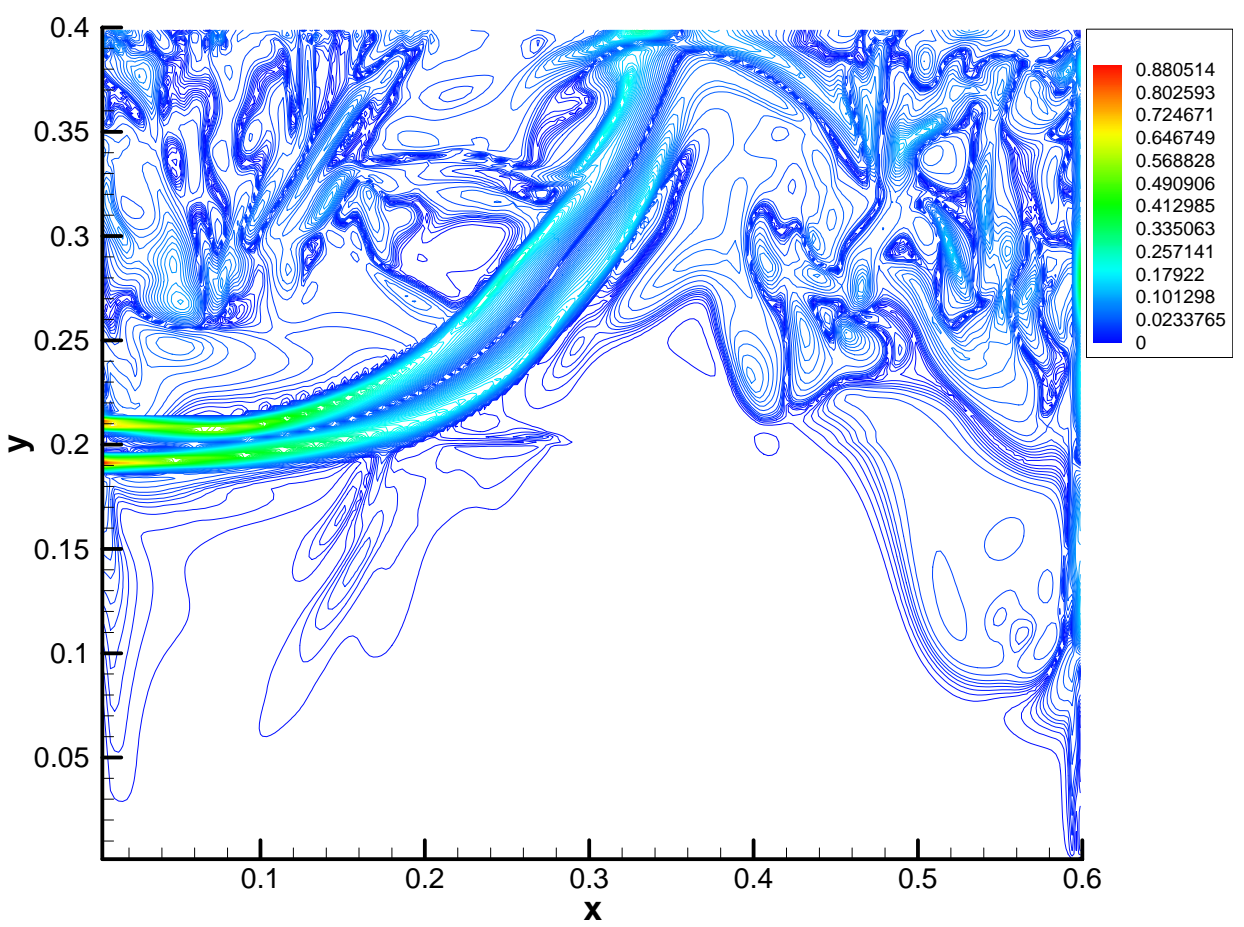

Figure 7.15 - Typical instantaneous vorticity magnitude contours for $240 \times 160 x 80$ grid; $\mathrm{dt}=0.001 ; \mathrm{U}_{\text {inlet }}=1.0 \mathrm{~m} / \mathrm{sec} ; \mathrm{t}^{*}=0.15 ; \mathrm{Fr}=7.65$ 


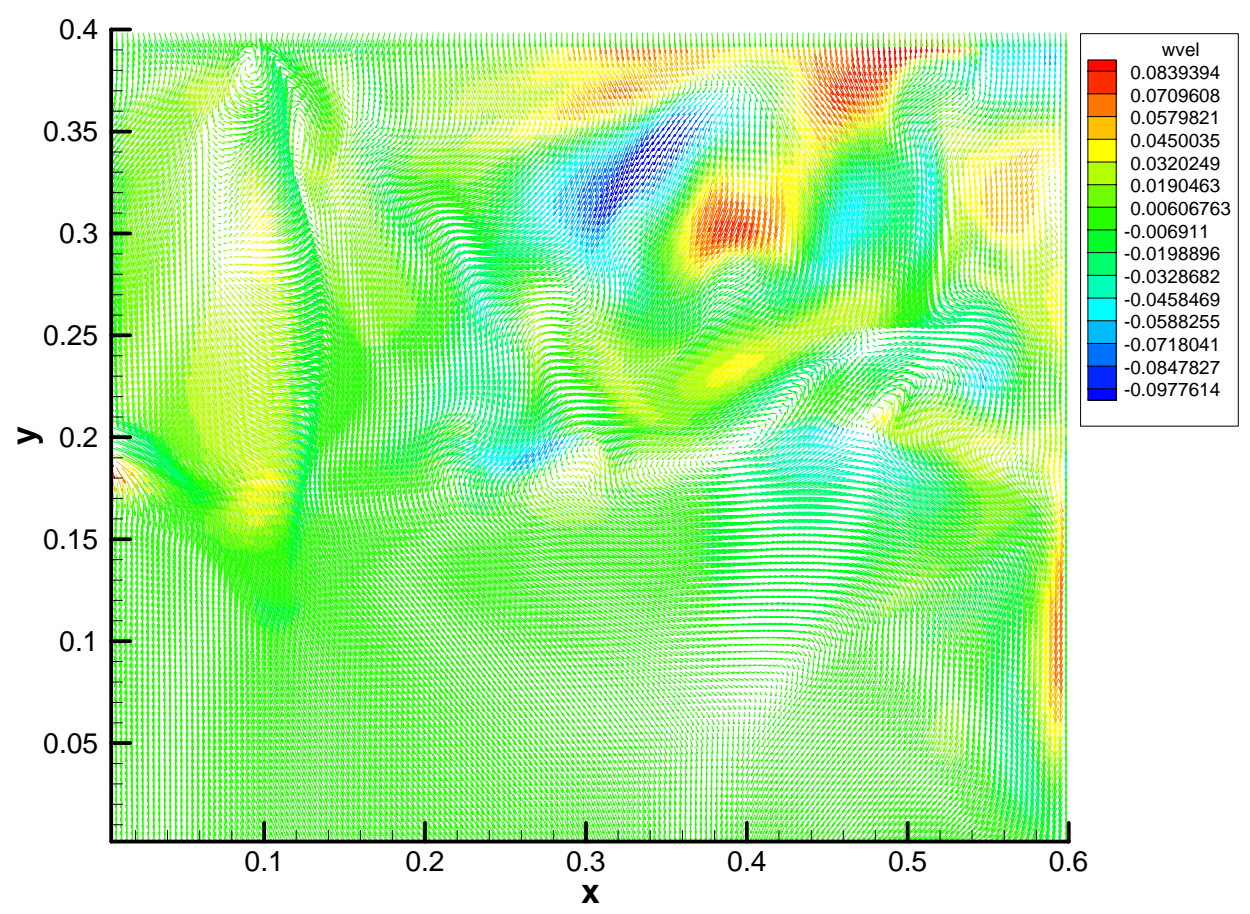

Figure 7.16 - Typical instantaneous velocity vectors (colored by span wise velocity component) for $240 \times 160 \times 80$ grid; $d t=0.001 ; U_{\text {inlet }}=0.25 \mathrm{~m} / \mathrm{sec} ; \mathrm{t}^{*}=0.2975 ; \mathrm{Fr}=$ 1.91

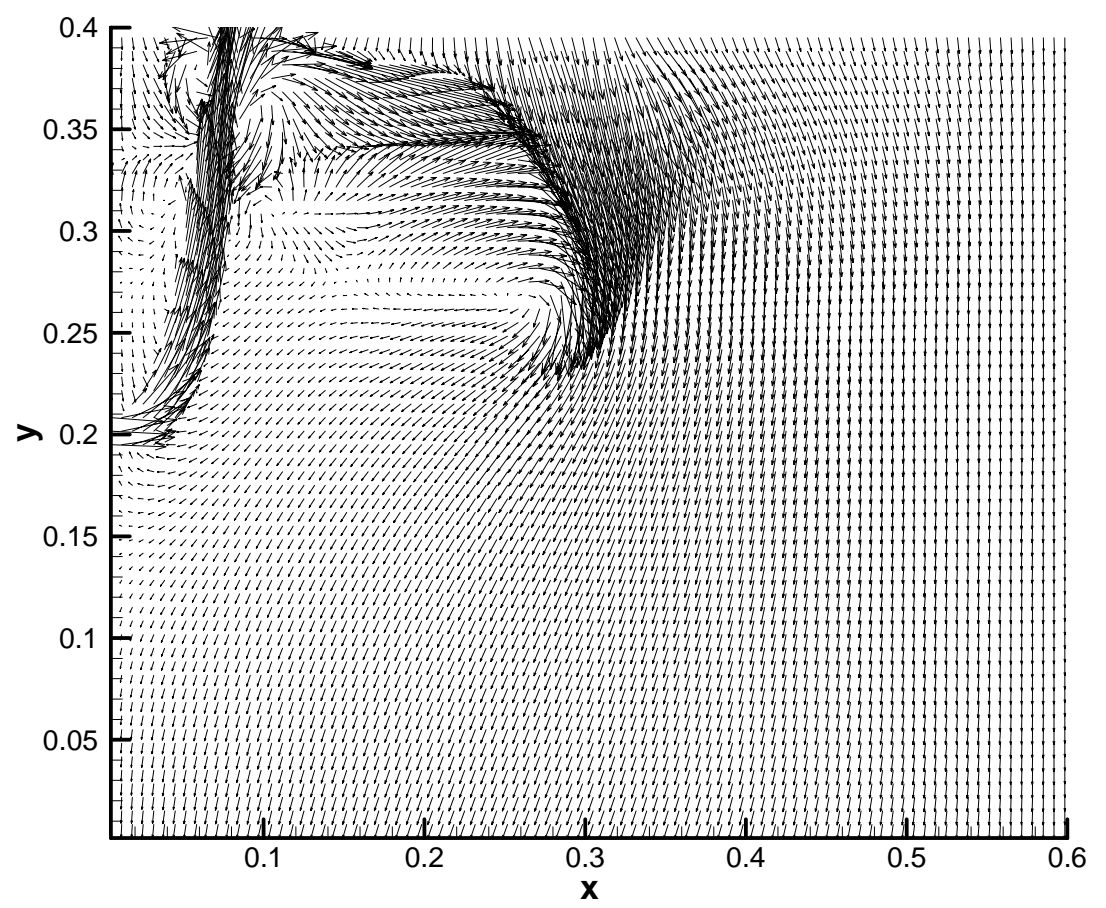

Figure 7.17 - Typical instantaneous velocity vectors for $240 \times 160 \times 80$ grid; $d t=0.001$; $U_{\text {inlet }}=0.25 \mathrm{~m} / \mathrm{sec} ; t^{*}=0.0982 ; \mathrm{Fr}=1.91$ 


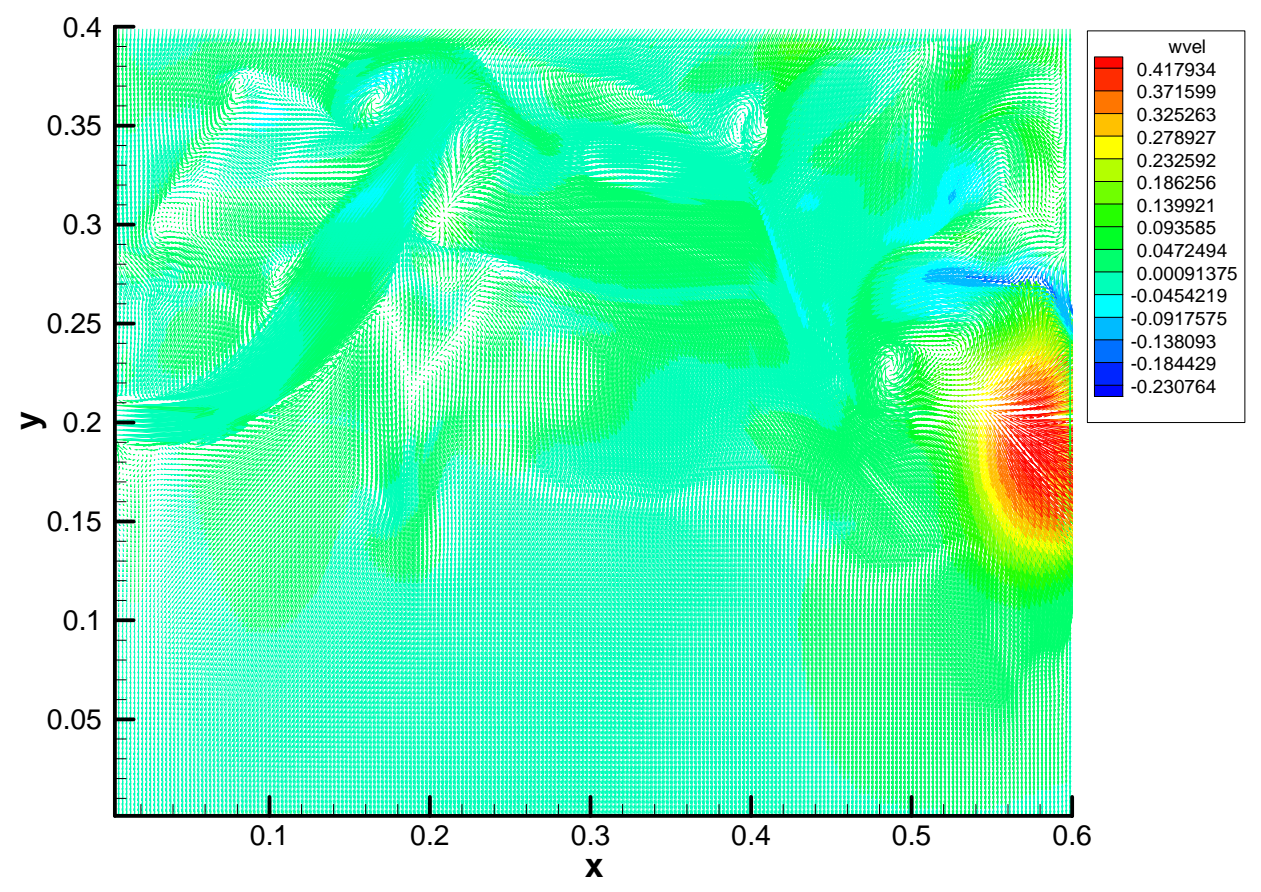

Figure 7.18 - Typical instantaneous velocity vectors (colored by span wise velocity component) for $240 \times 160 \times 80$ grid; $d t=0.001 ; U_{\text {inlet }}=0.5 \mathrm{~m} / \mathrm{sec} ; \mathrm{t}^{*}=0.3875 ; \mathrm{Fr}=3.83$

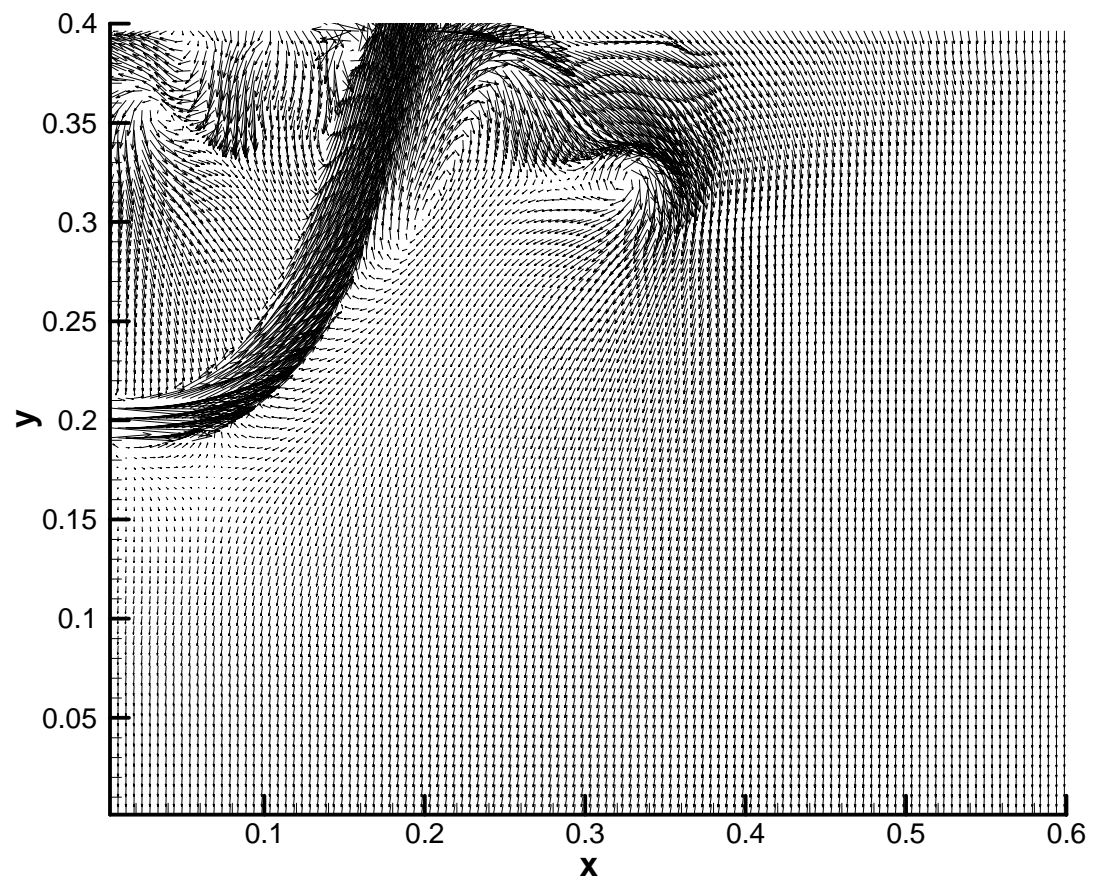

Figure 7.19 - Typical instantaneous velocity vectors for $240 \times 160 \times 80$ grid; $d t=0.001$; $U_{\text {inlet }}=0.5 \mathrm{~m} / \mathrm{sec} ; \mathrm{t}^{*}=\mathbf{0 . 1 0 7 5} ; \mathrm{Fr}=3.83$ 


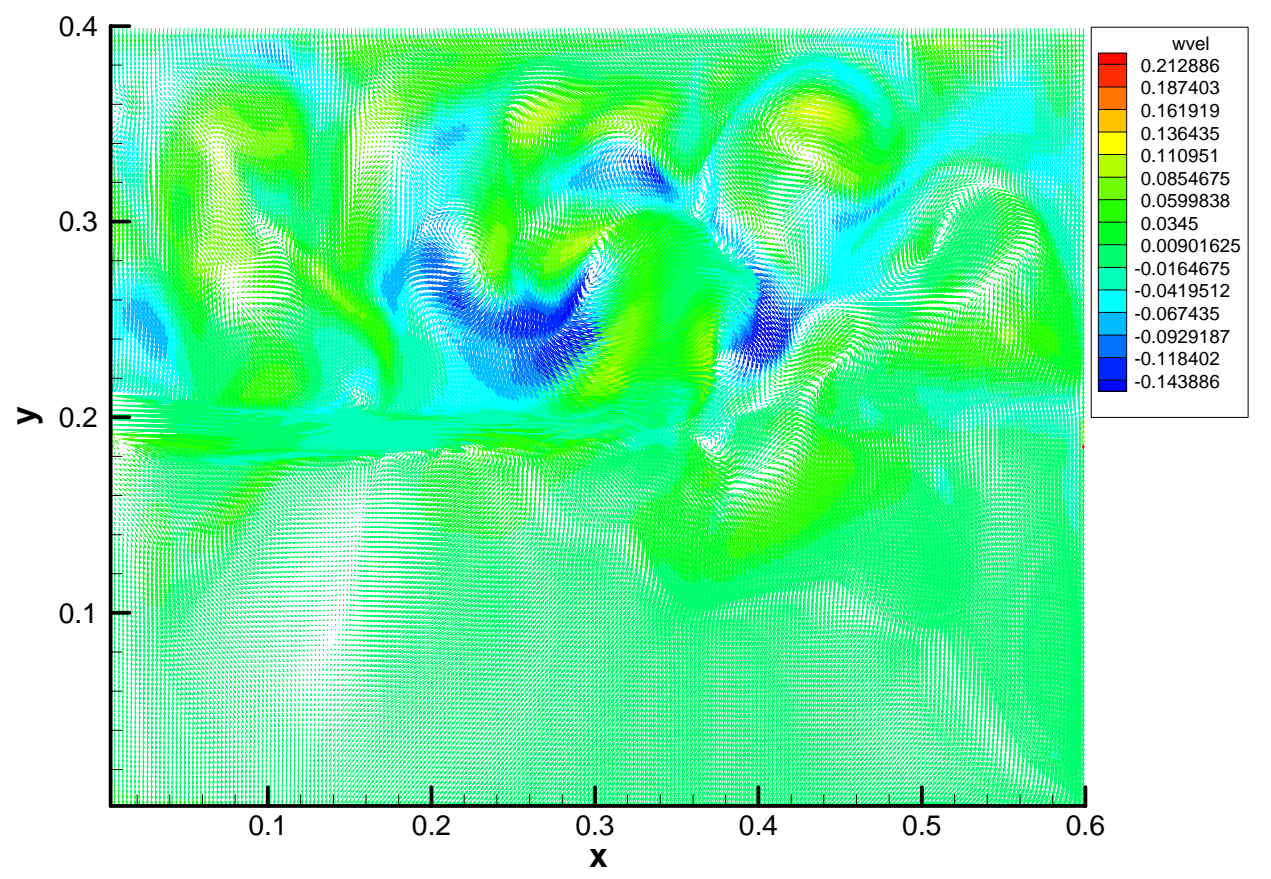

Figure 7.20 - Typical instantaneous velocity vectors (colored by span wise velocity component) for $240 \times 160 \times 80$ grid; $d t=0.001 ; U_{\text {inlet }}=1.0 \mathrm{~m} / \mathrm{sec} ; \mathrm{t}^{*}=0.15 ; \mathrm{Fr}=7.65$

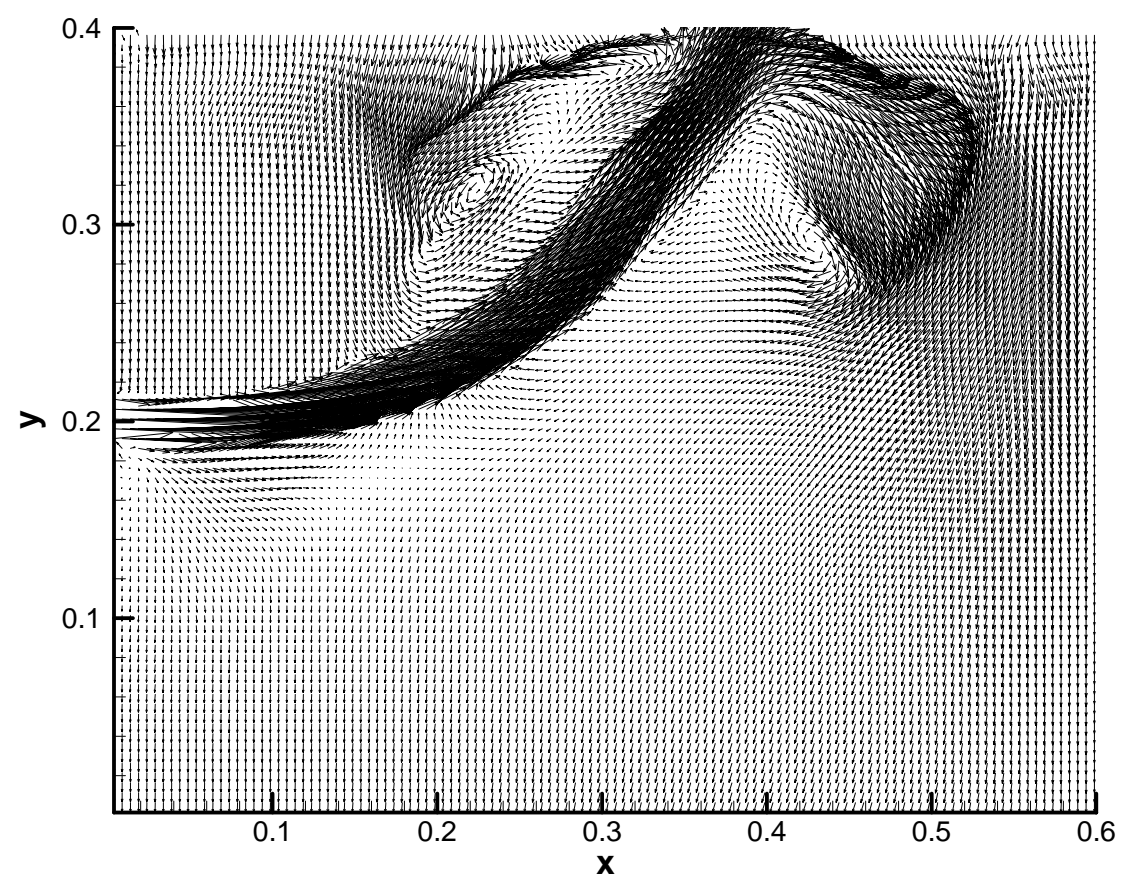

Figure 7.21 - Typical instantaneous velocity vectors for $240 \times 160 \times 80$ grid; $\mathrm{dt}=0.001$; $U_{\text {inlet }}=1.0 \mathrm{~m} / \mathrm{sec} ; \mathrm{t}^{*}=0.085 ; \mathrm{Fr}=7.65$ 


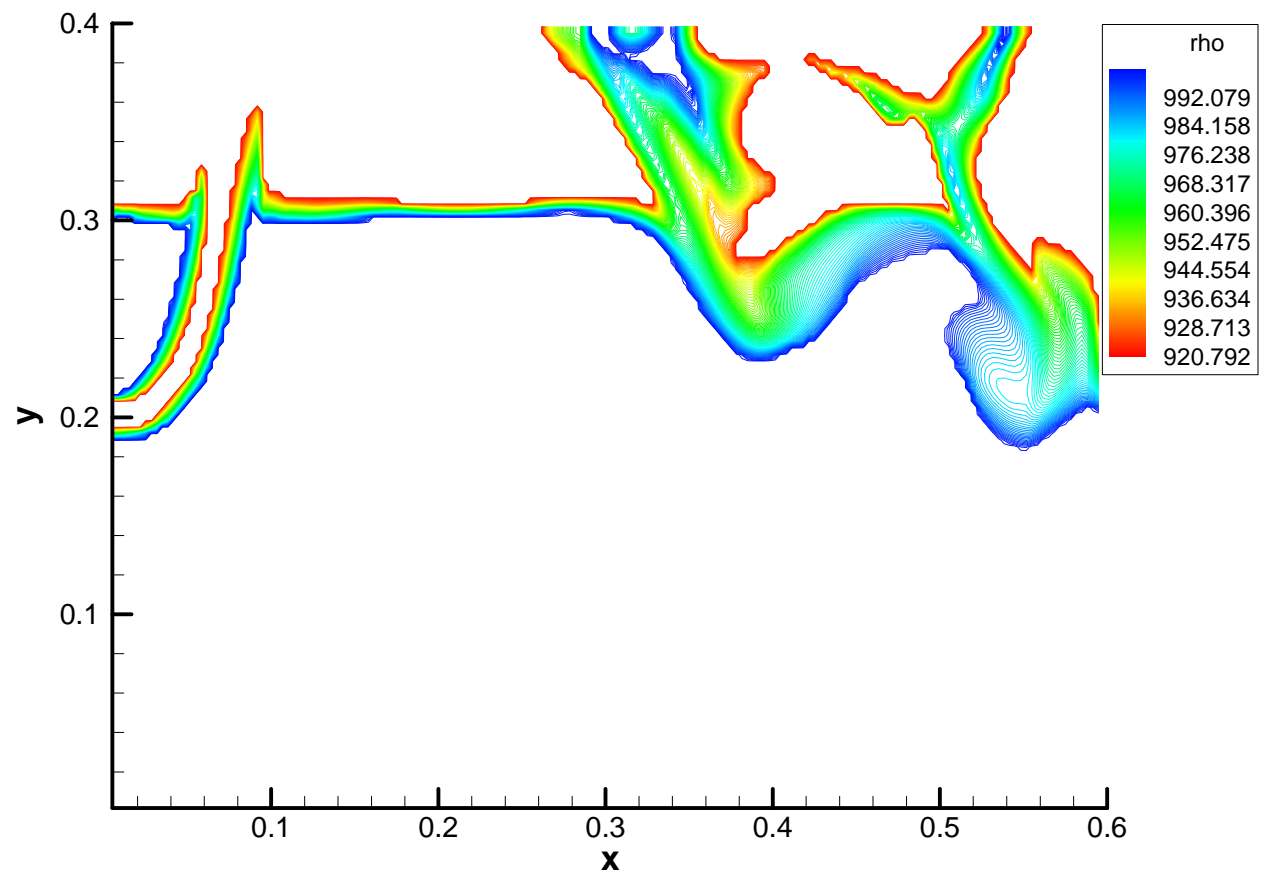

Figure 7.22 - Typical instantaneous density contours for 240x160x80 grid; $\mathrm{dt}=$ $0.001 ; U_{\text {inlet }}=0.25 \mathrm{~m} / \mathrm{sec} ; \mathrm{t}^{*}=0.1085 ; \mathrm{Fr}=1.91$

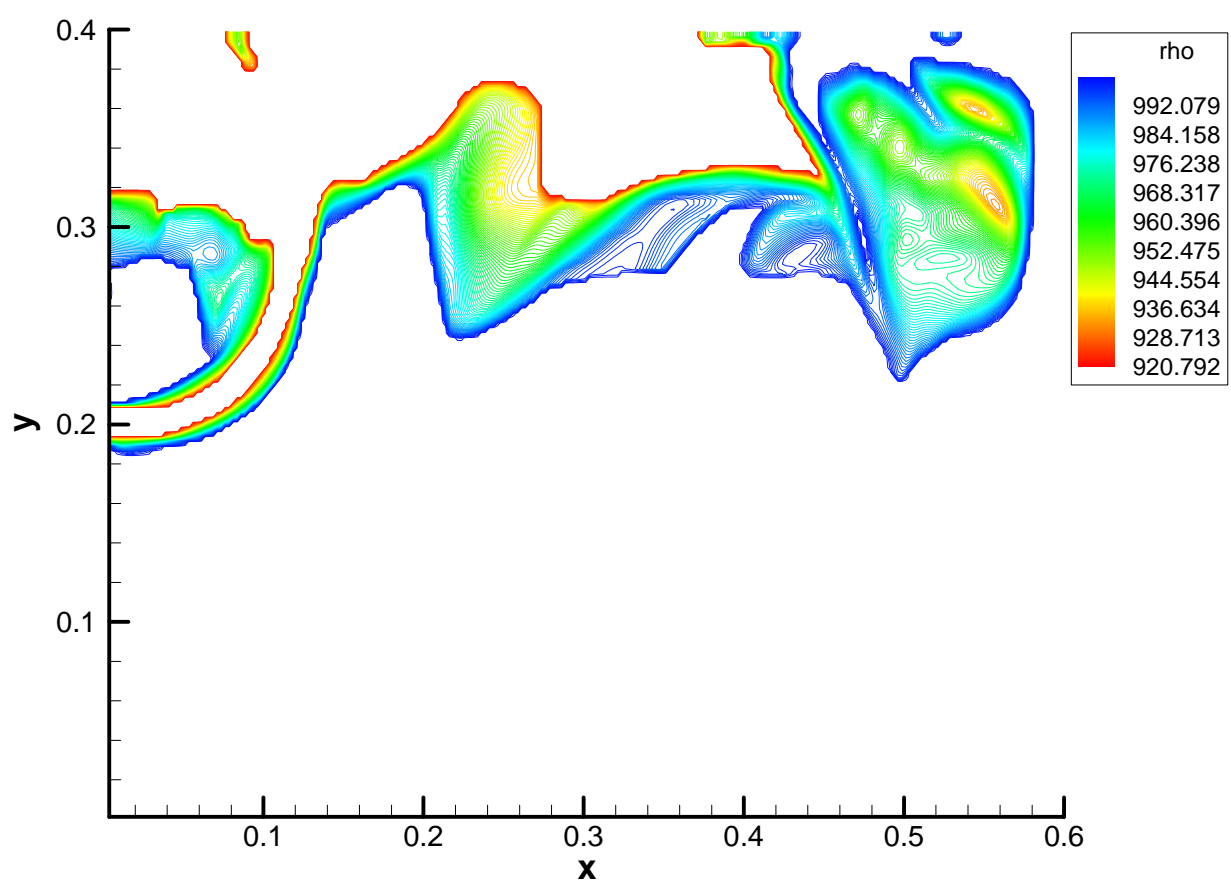

Figure 7.23 - Typical instantaneous density contours for 240x160x80 grid; $\mathrm{dt}=$ $0.001 ; U_{\text {inlet }}=0.5 \mathrm{~m} / \mathrm{sec} ; \mathrm{t}^{*}=0.275 ; \mathrm{Fr}=3.63$ 


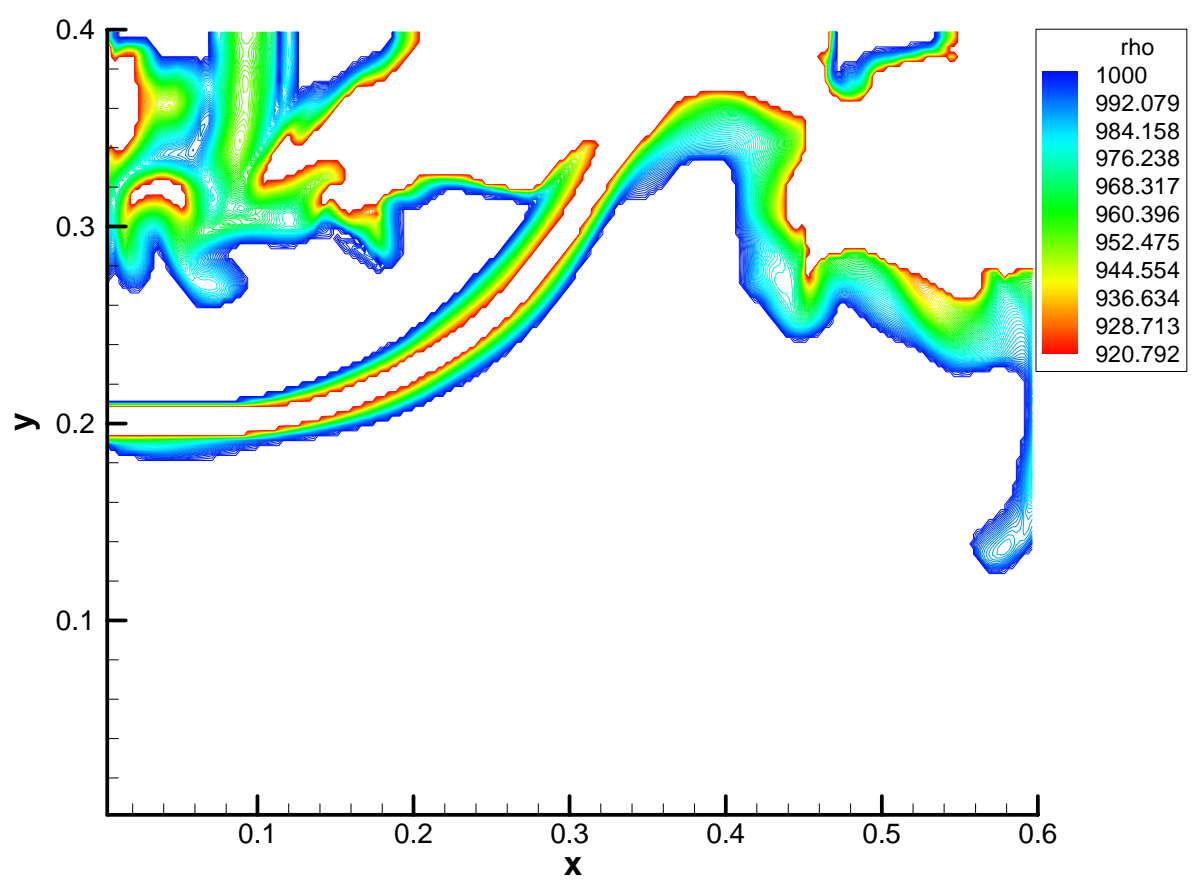

Figure 7.24 - Typical instantaneous density contours for 240x160x80 grid; $\mathrm{dt}=$ $0.001 ; U_{\text {inlet }}=1.0 \mathrm{~m} / \mathrm{sec} ; t^{*}=0.263$ seconds; $F r=7.65$ 


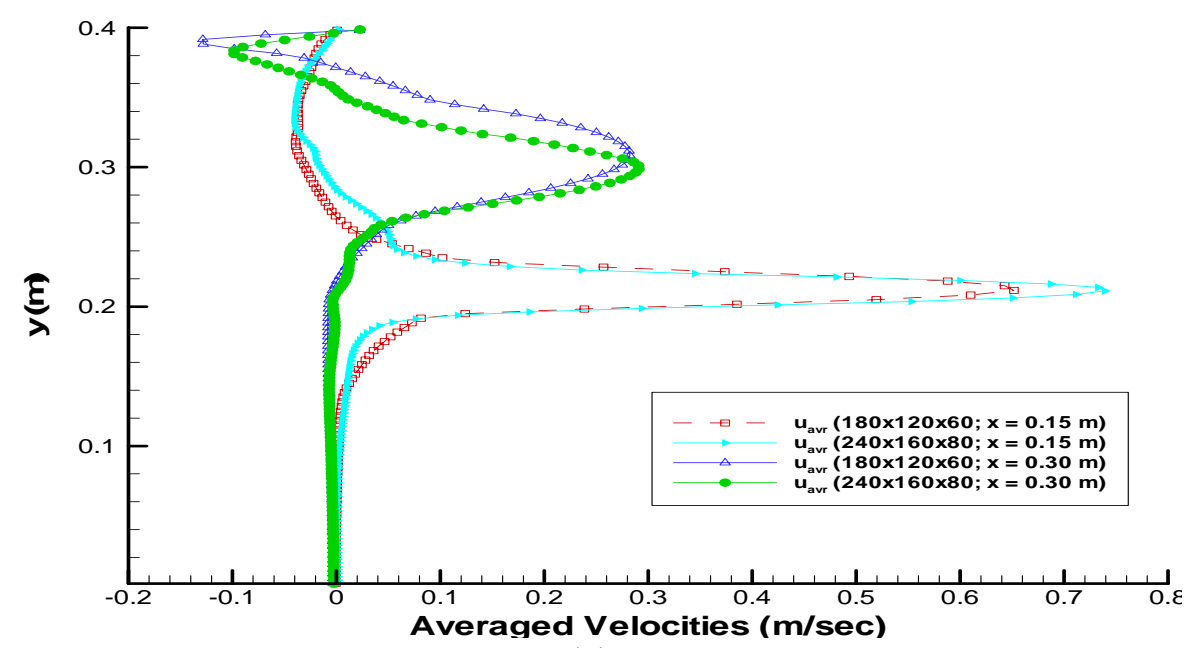

(a)

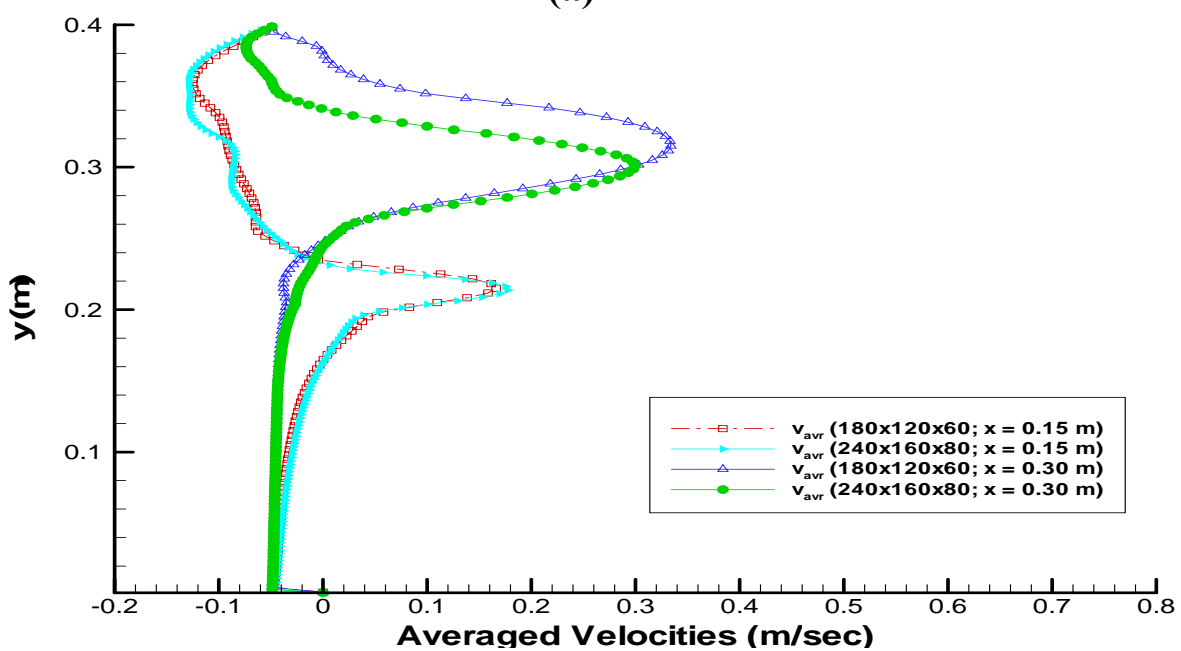

(b)

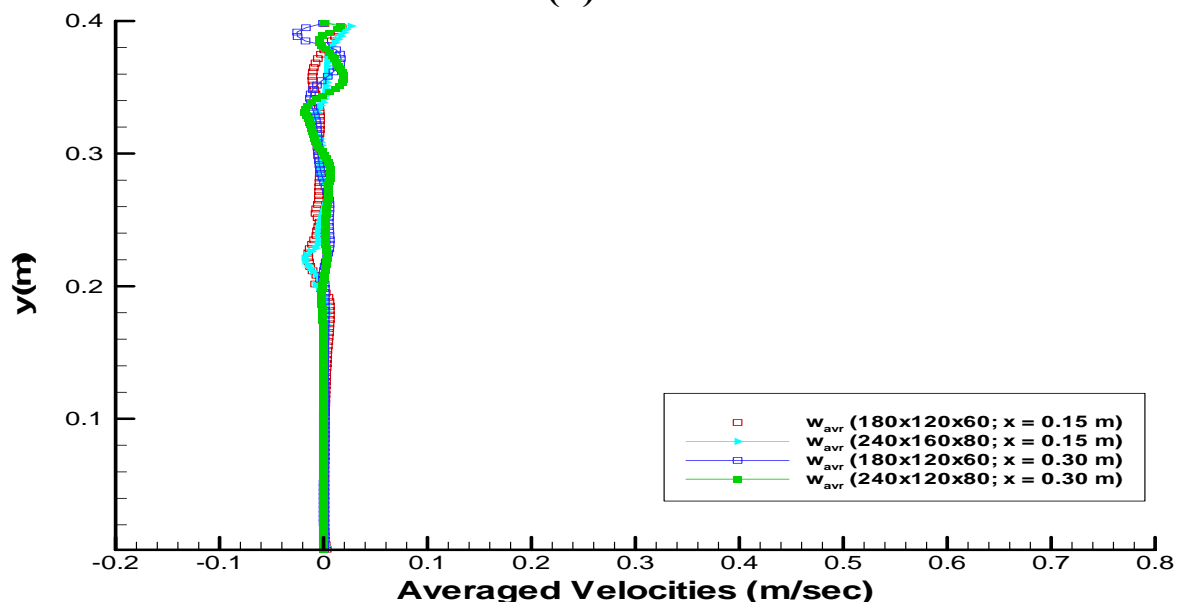

(c)

Figure 7.25 - Average velocity profiles from DREAM ${ }^{\circledR}$ simulations at $x=0.15 \mathrm{~m}$ and $0.30 \mathrm{~m}$ using $180 \times 120 \times 60$ and $240 \times 160 \times 80$ for a) axial velocity component, b) vertical velocity component, and c) spanwise velocity component; $d t=0.001 ; U_{\text {inlet }}=1.0$ $\mathrm{m} / \mathrm{sec} ; \mathrm{Fr}=\mathbf{7 . 6 5}$ 


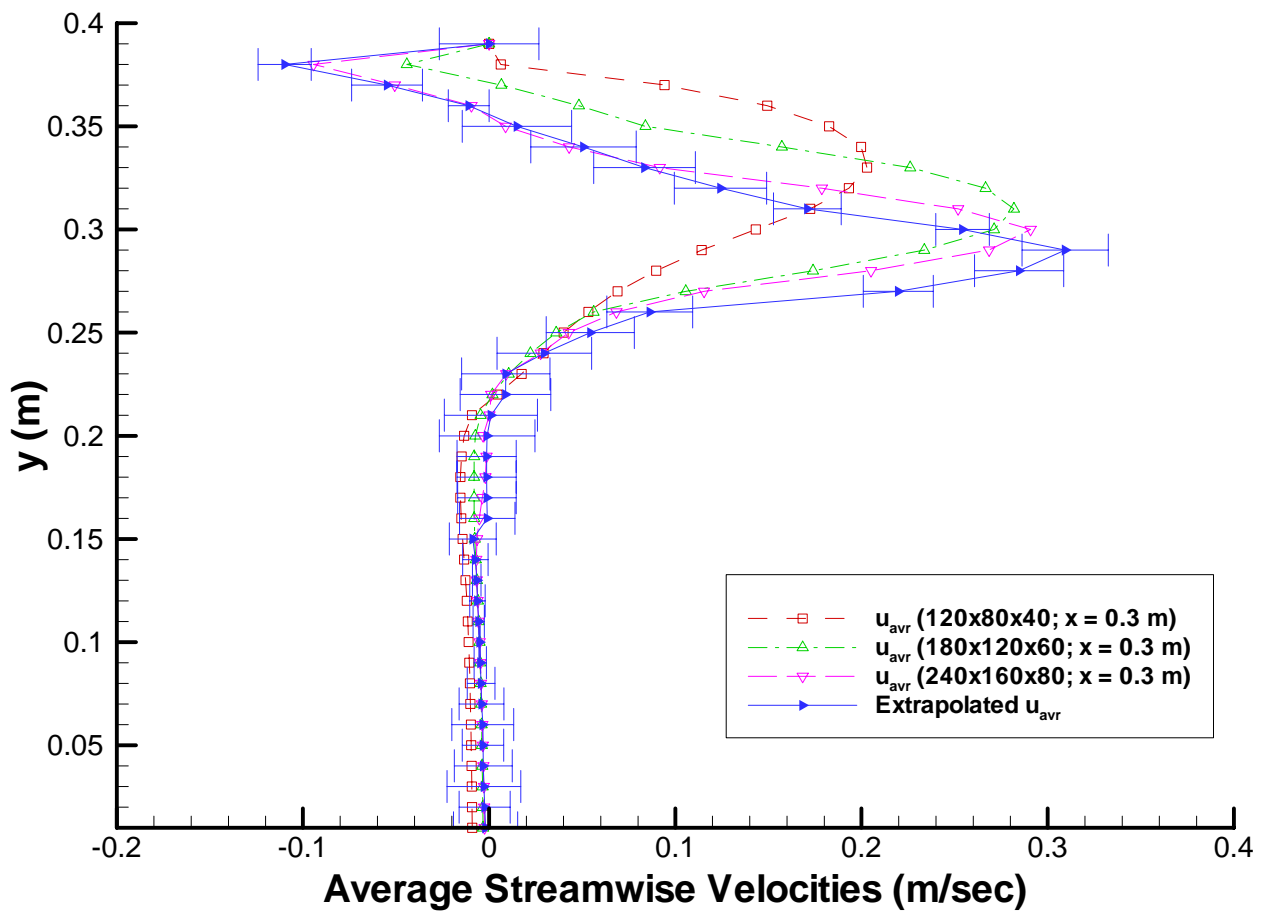

Figure 7.26 - Richardson extrapolation for average axial velocity profiles at $x=0.30$ $m$ for $120 \times 80 \times 40,180 \times 120 \times 60$ and $240 \times 160 \times 80$ grids; $d t=0.001 ; U_{\text {inlet }}=1.0 \mathrm{~m} / \mathrm{sec}$; $\mathrm{Fr}=7.65$ 


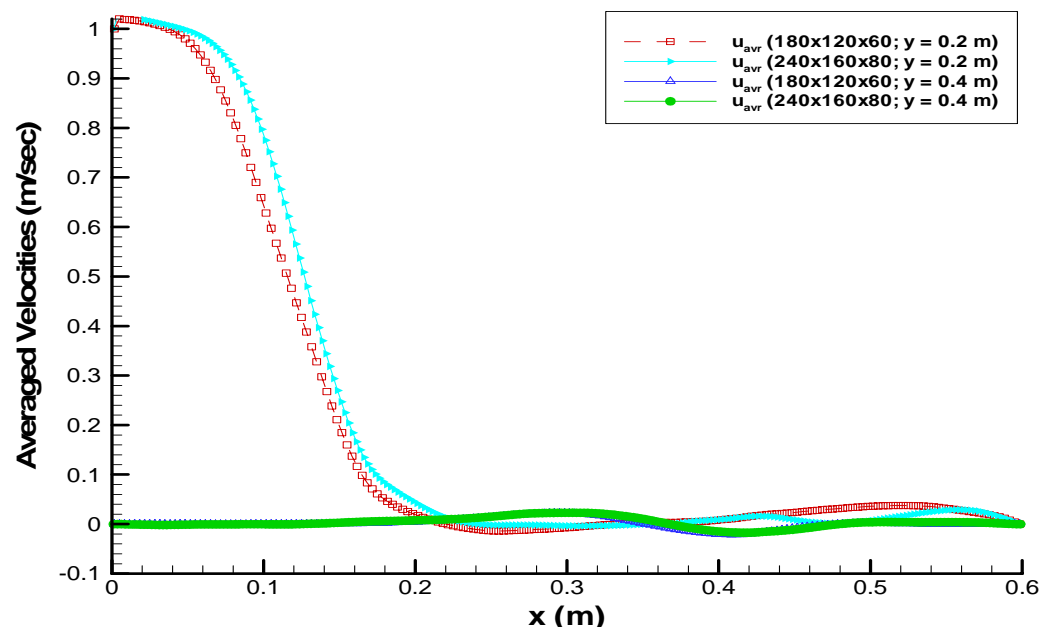

(a)

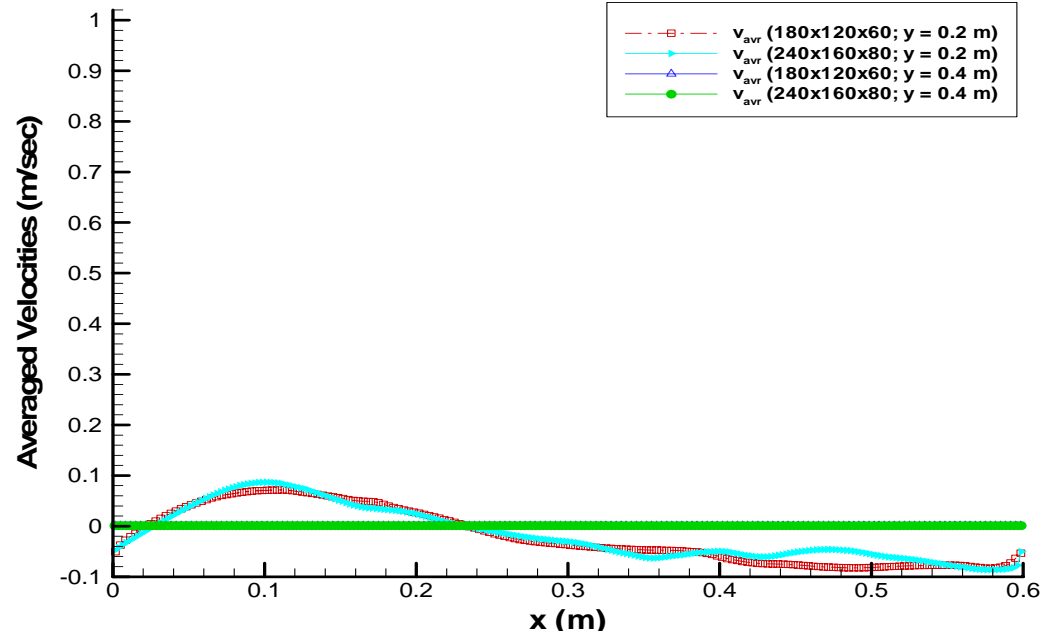

(b)

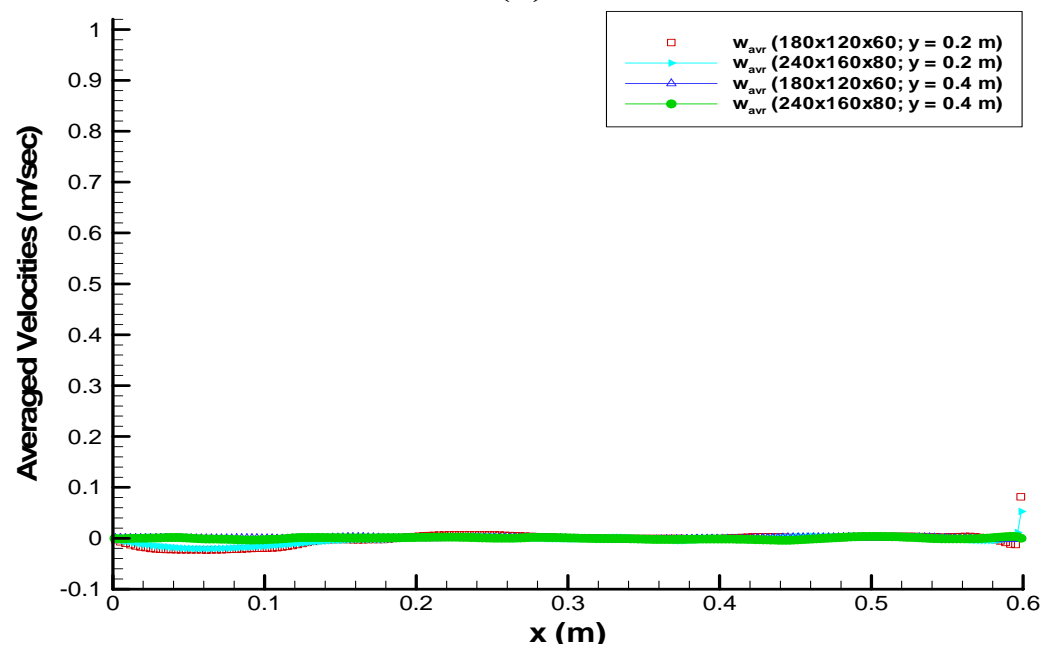

(c)

Figure 7.27 - Average velocity profiles from DREAM ${ }^{\circledR}$ simulations at $\mathbf{y}=0.2 \mathrm{~m}$ and $0.4 \mathrm{~m}$ using $180 \times 120 \times 60$ and $240 \times 160 \times 80$ for a) axial velocity component, b) vertical velocity component, and c) spanwise velocity component; $d t=0.001 ; U_{\text {inlet }}=1.0$ $\mathrm{m} / \mathrm{sec} ; \mathrm{Fr}=7.65$ 


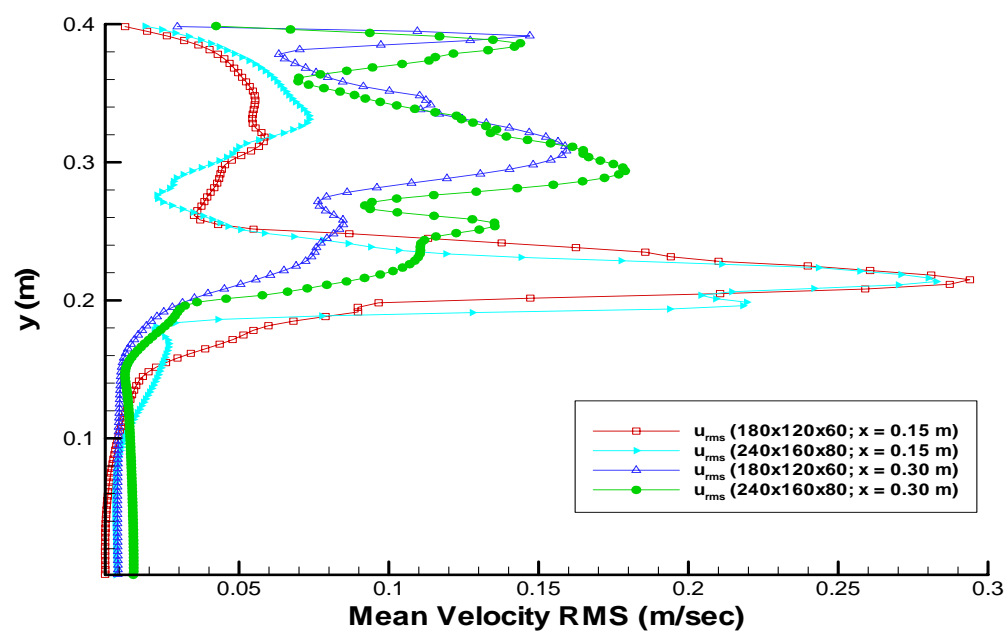

(a)

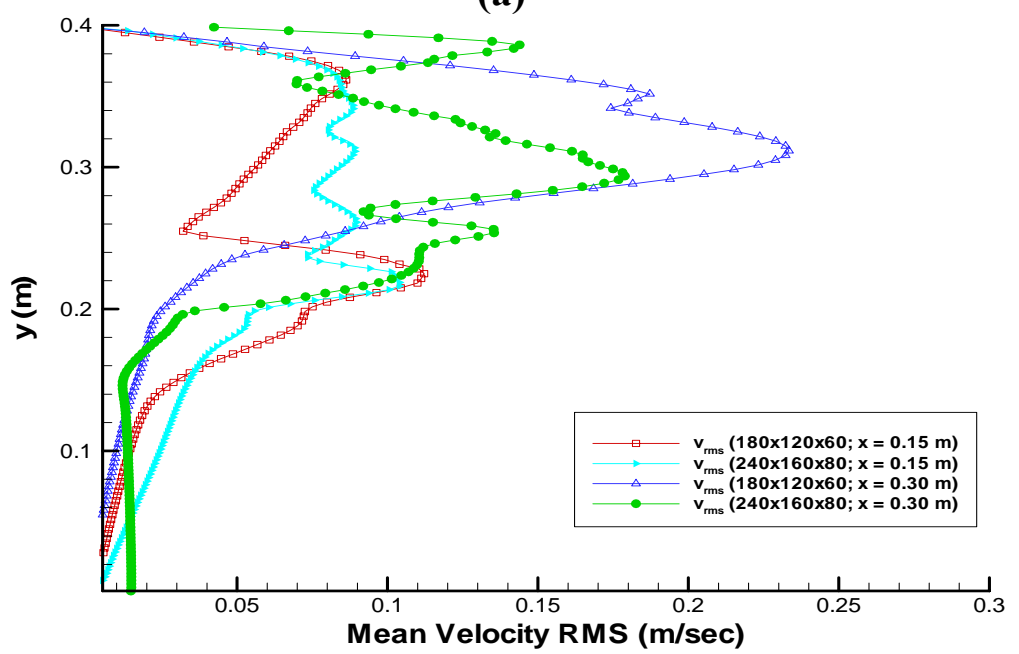

(b)

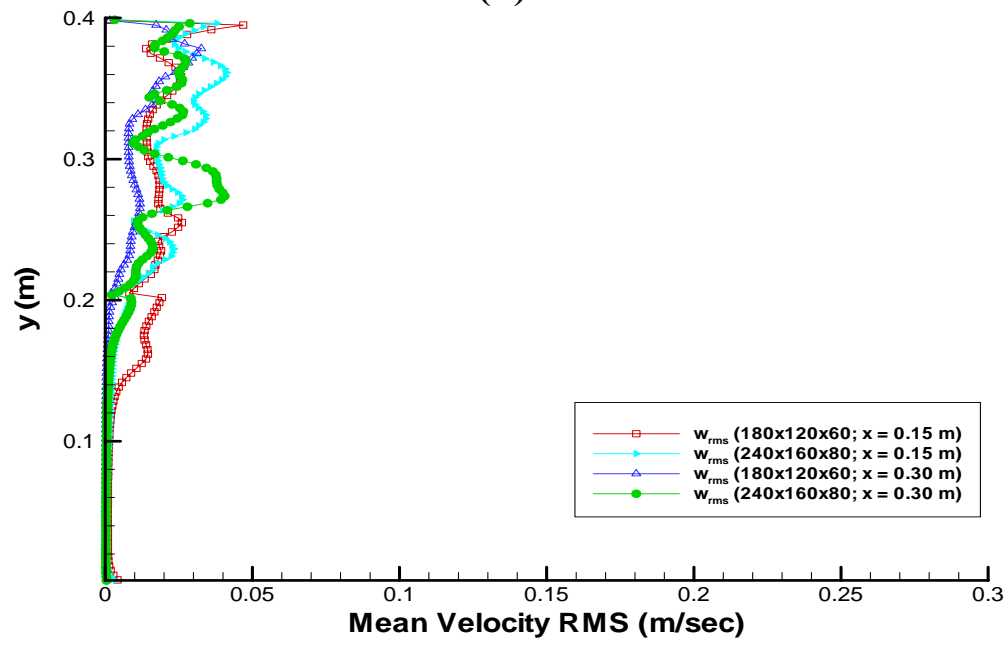

(c)

Figure 7.28 - Mean RMS velocity profiles from DREAM ${ }^{\circledR}$ simulations at $x=0.15$ m and $0.30 \mathrm{~m}$ using $180 \times 120 \times 60$ and $240 \times 160 \times 80$ for a) axial velocity component, b) vertical velocity component, and c) spanwise velocity component; $\mathrm{dt}=0.001 ; \mathrm{U}_{\text {inlet }}=$ $1.0 \mathrm{~m} / \mathrm{sec} ; \mathrm{Fr}=7.65$ 


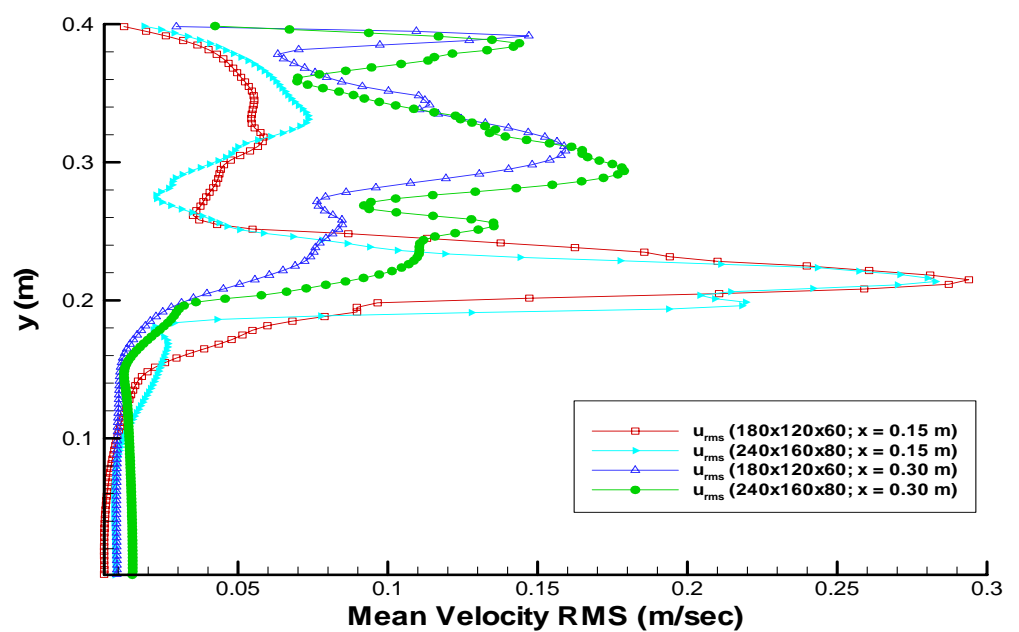

(a)

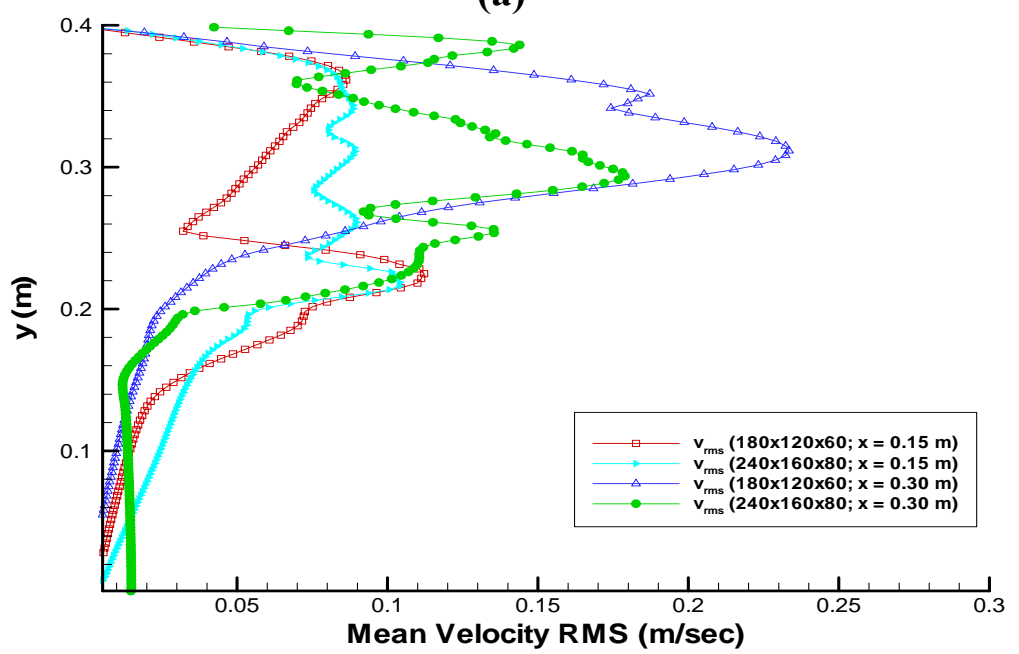

(b)

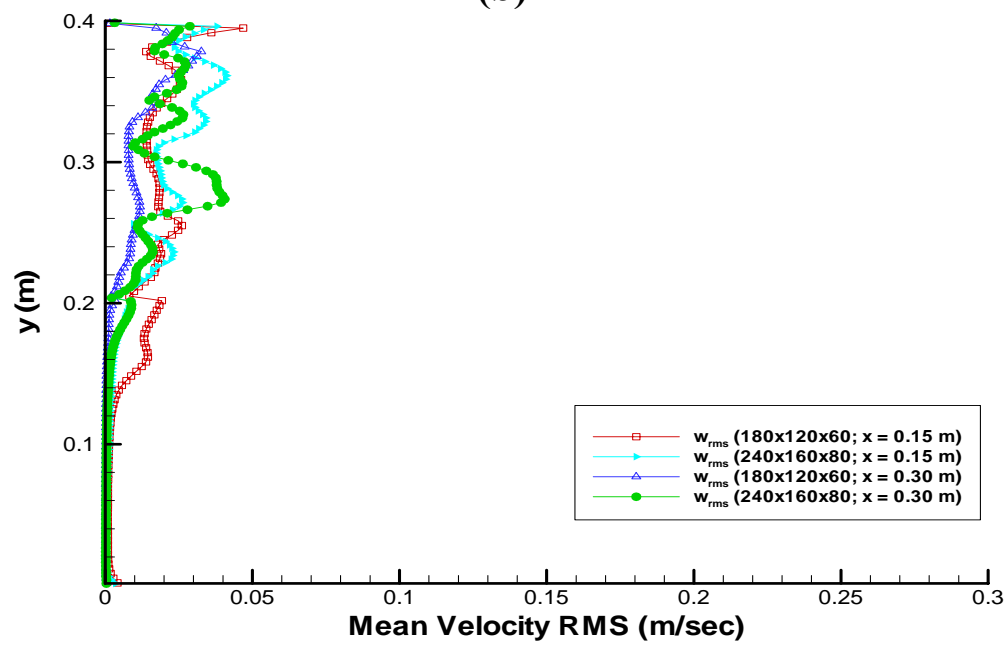

(c)

Figure 7.29 - Mean RMS velocity profiles from DREAM ${ }^{\circledR}$ simulations at $y=0.2 \mathrm{~m}$ and $0.40 \mathrm{~m}$ using $180 \times 120 \times 60$ and $240 \times 160 \times 80$ for a) axial velocity component, b) vertical velocity component, and c) spanwise velocity component; $\mathrm{dt}=0.001 ; \mathrm{U}_{\text {inlet }}=$ $1.0 \mathrm{~m} / \mathrm{sec} ; \mathrm{Fr}=7.65$ 


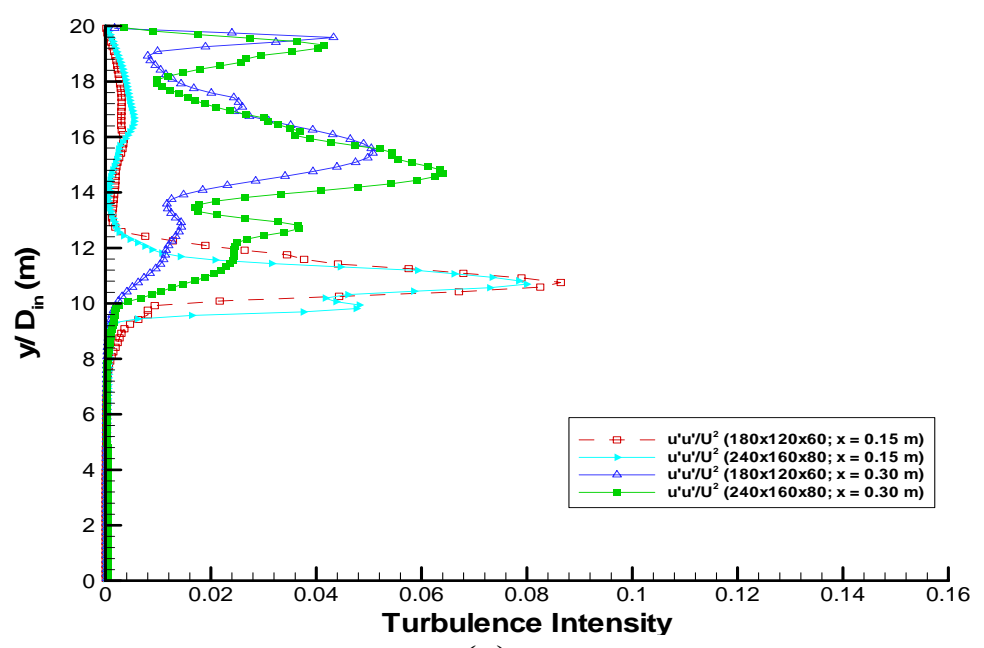

(a)

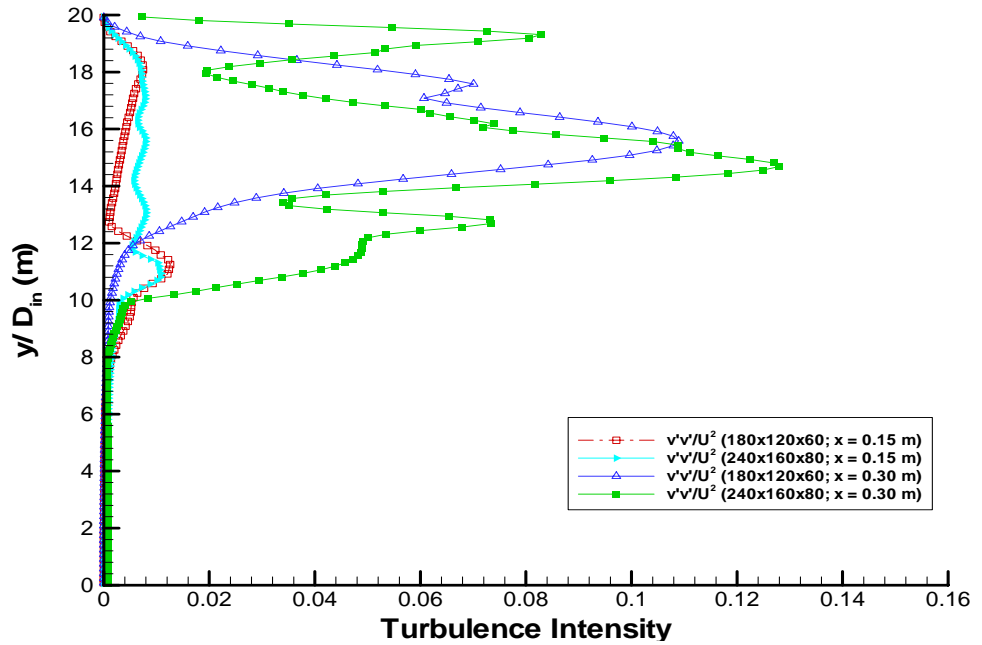

(b)

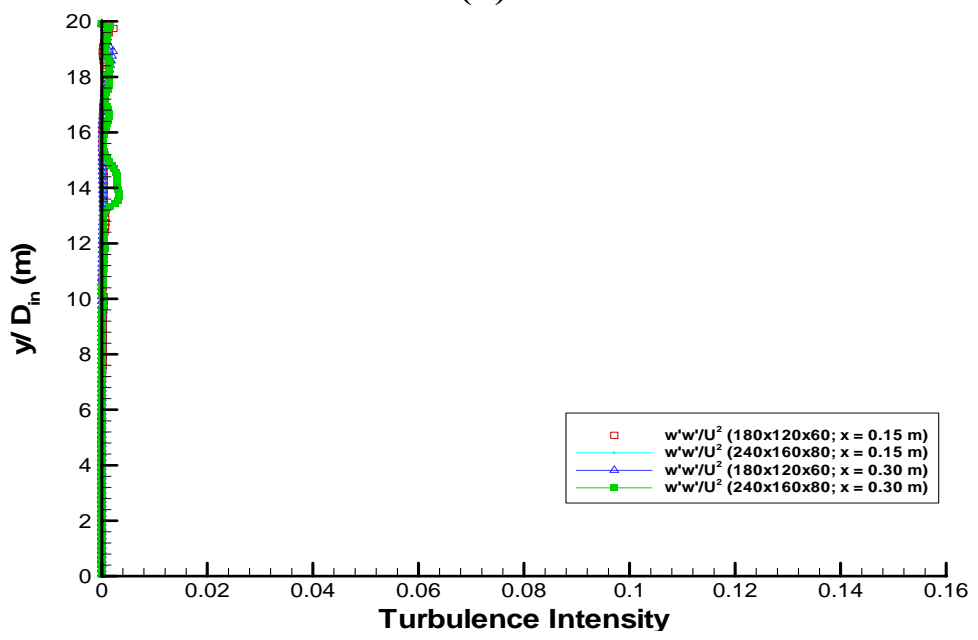

(c)

Figure 7.30 - Square of the turbulence intensity from DREAM ${ }^{\circledR}$ simulations at $x=$ $0.15 \mathrm{~m}$ and $0.30 \mathrm{~m}$ using $180 \times 120 \times 60$ and $240 \times 160 \times 80$ for a) axial velocity component, b) vertical velocity component, and c) spanwise velocity component; $d t$ $=0.001 ; U_{\text {inlet }}=1.0 \mathrm{~m} / \mathrm{sec} ; \mathrm{Fr}=7.65$ 


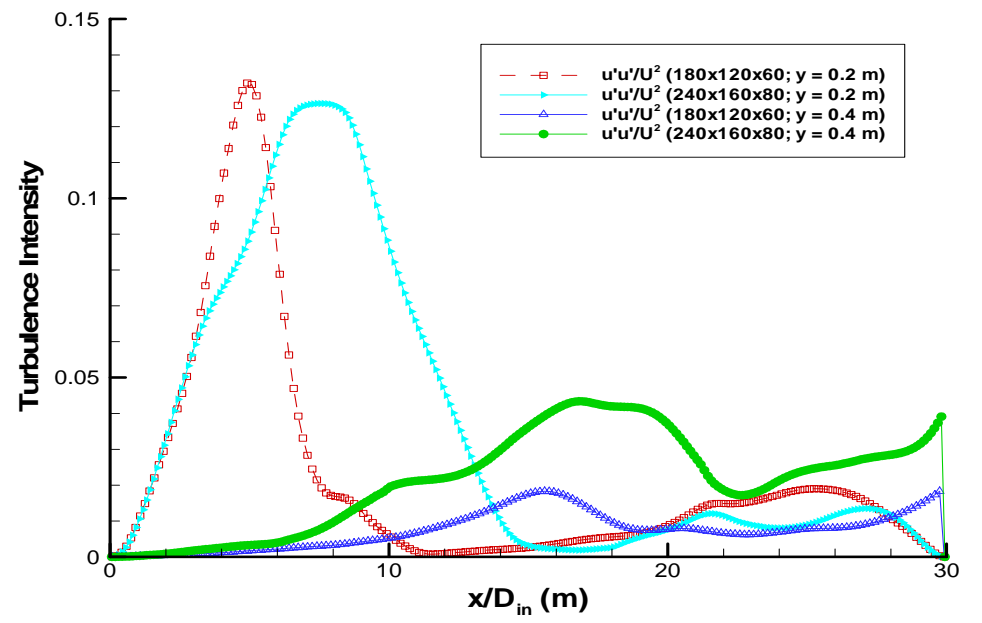

(a)

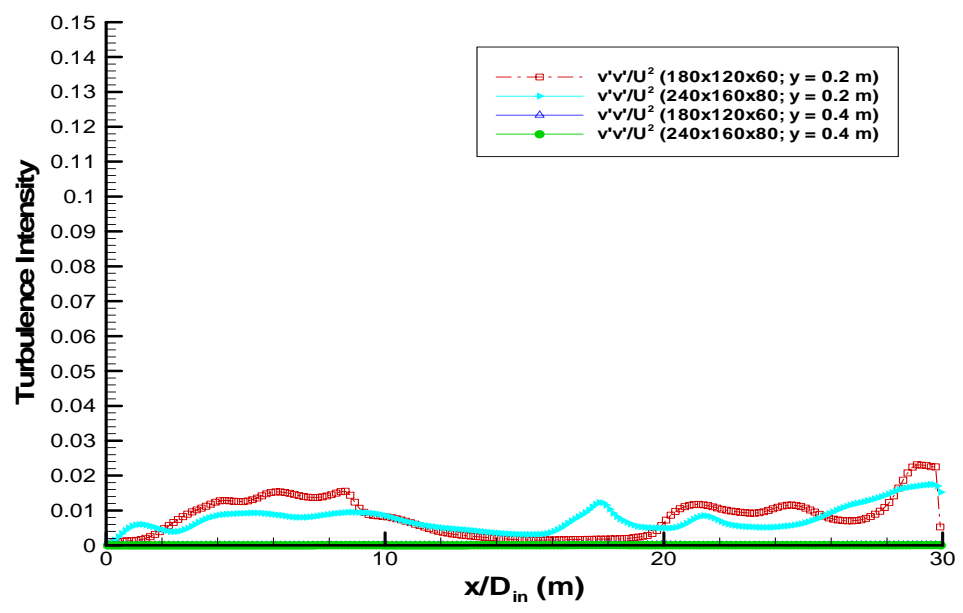

(b)

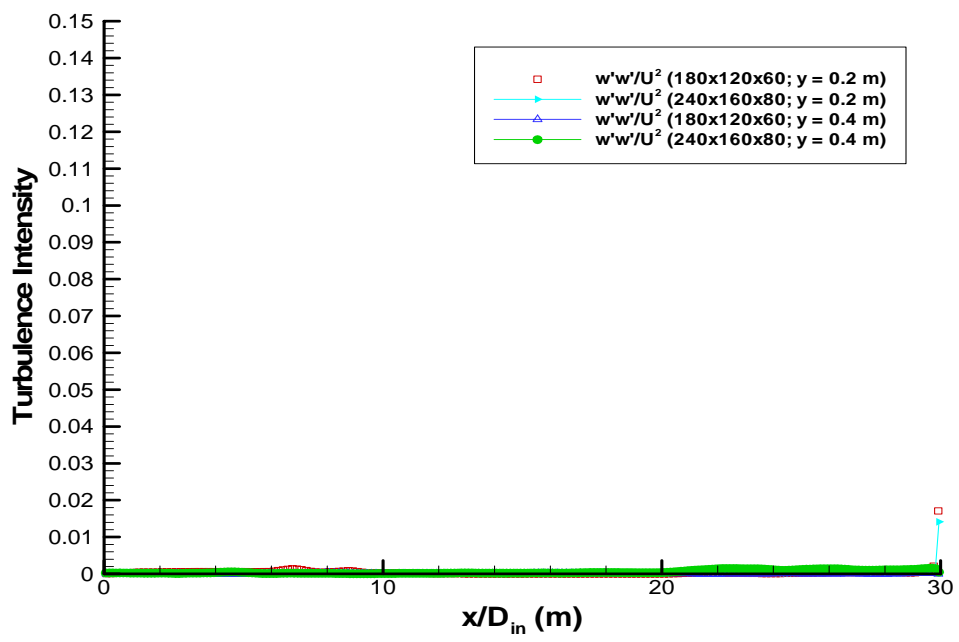

(c)

Figure 7.31 - Square of the turbulence intensity from DREAM ${ }^{\circledR}$ simulations at $y=$ $0.2 \mathrm{~m}$ and $0.40 \mathrm{~m}$ using $180 \times 120 \times 60$ and $240 \times 160 \times 80$ for a) axial velocity component, b) vertical velocity component, and c) spanwise velocity component; $d t=0.001 ; U_{\text {inlet }}$ $=1.0 \mathrm{~m} / \mathrm{sec} ; \mathrm{Fr}=7.65$ 


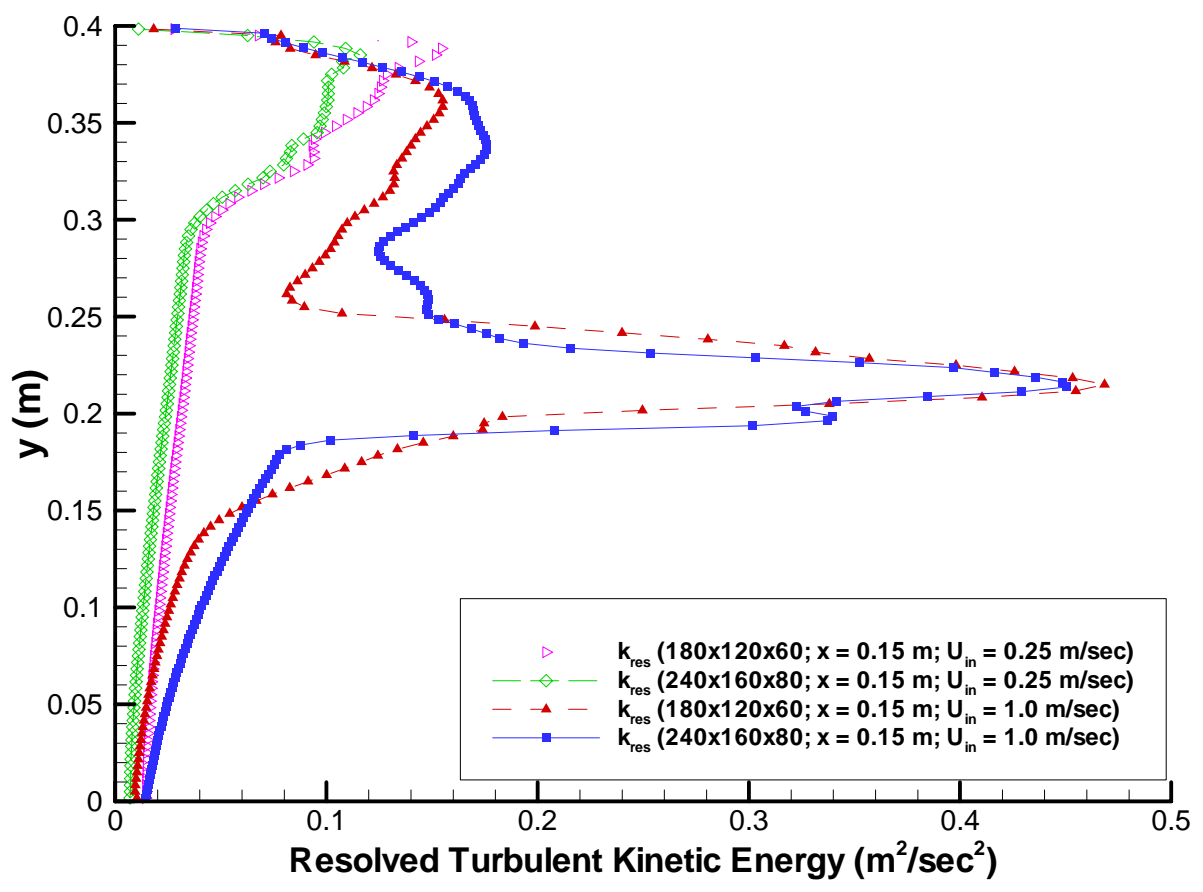

Figure 7.32 - Resolved turbulent kinetic energy at $x=0.15 \mathrm{~m}$ for 180x120x60 and $240 \times 160 \times 80$ grids; $d t=0.001 ; U_{\text {inlet }}=0.25$ and $1.0 \mathrm{~m} / \mathrm{sec} ; \mathrm{Fr}=1.91$ and 7.65

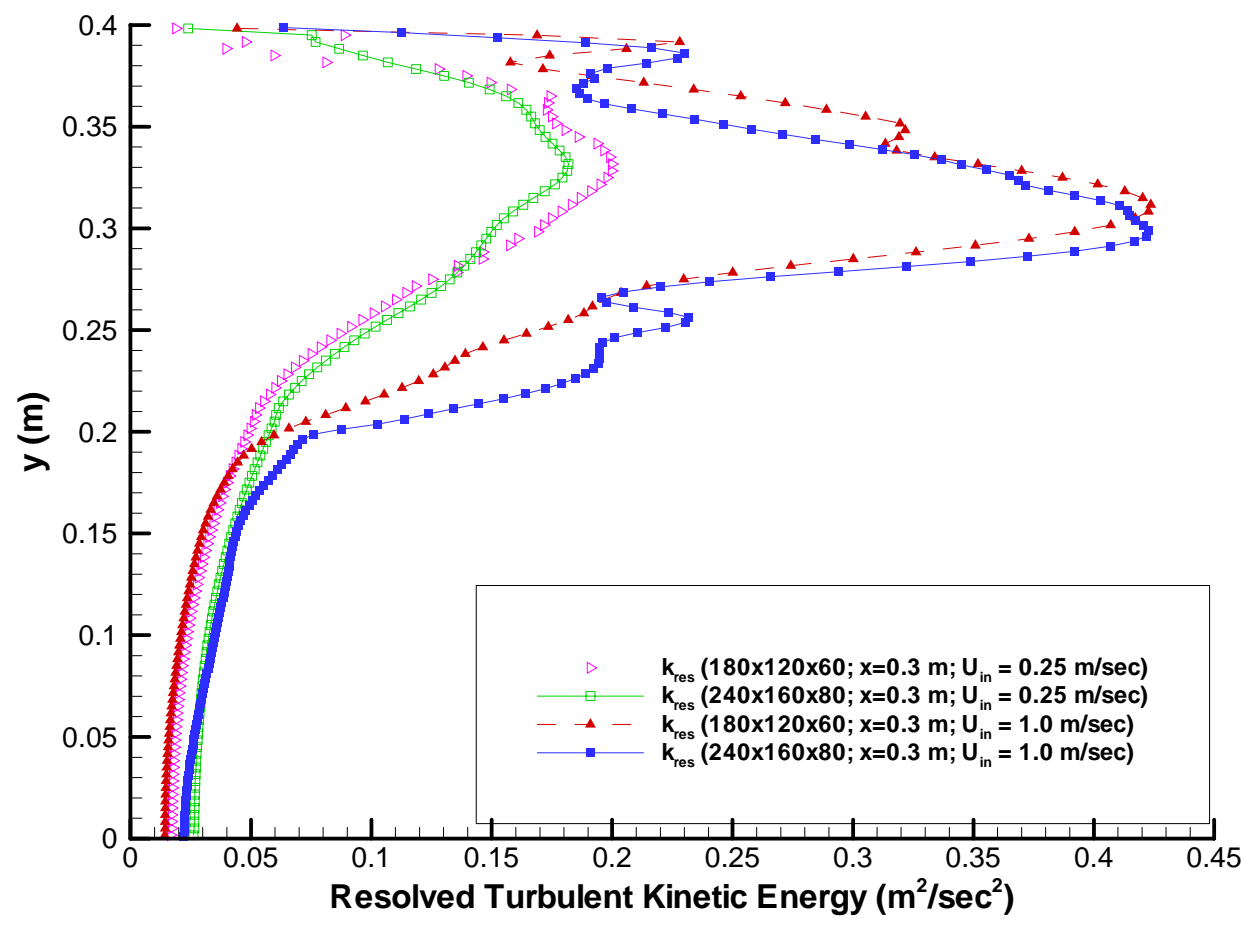

Figure 7.33 - Resolved turbulent kinetic energy at $x=0.30 \mathrm{~m}$ for $180 \times 120 \times 60$ and $240 \times 160 \times 80$ grids; $d t=0.001 ; U_{\text {inlet }}=0.25$ and $1.0 \mathrm{~m} / \mathrm{sec} ; \mathrm{Fr}=1.91$ and 7.65 


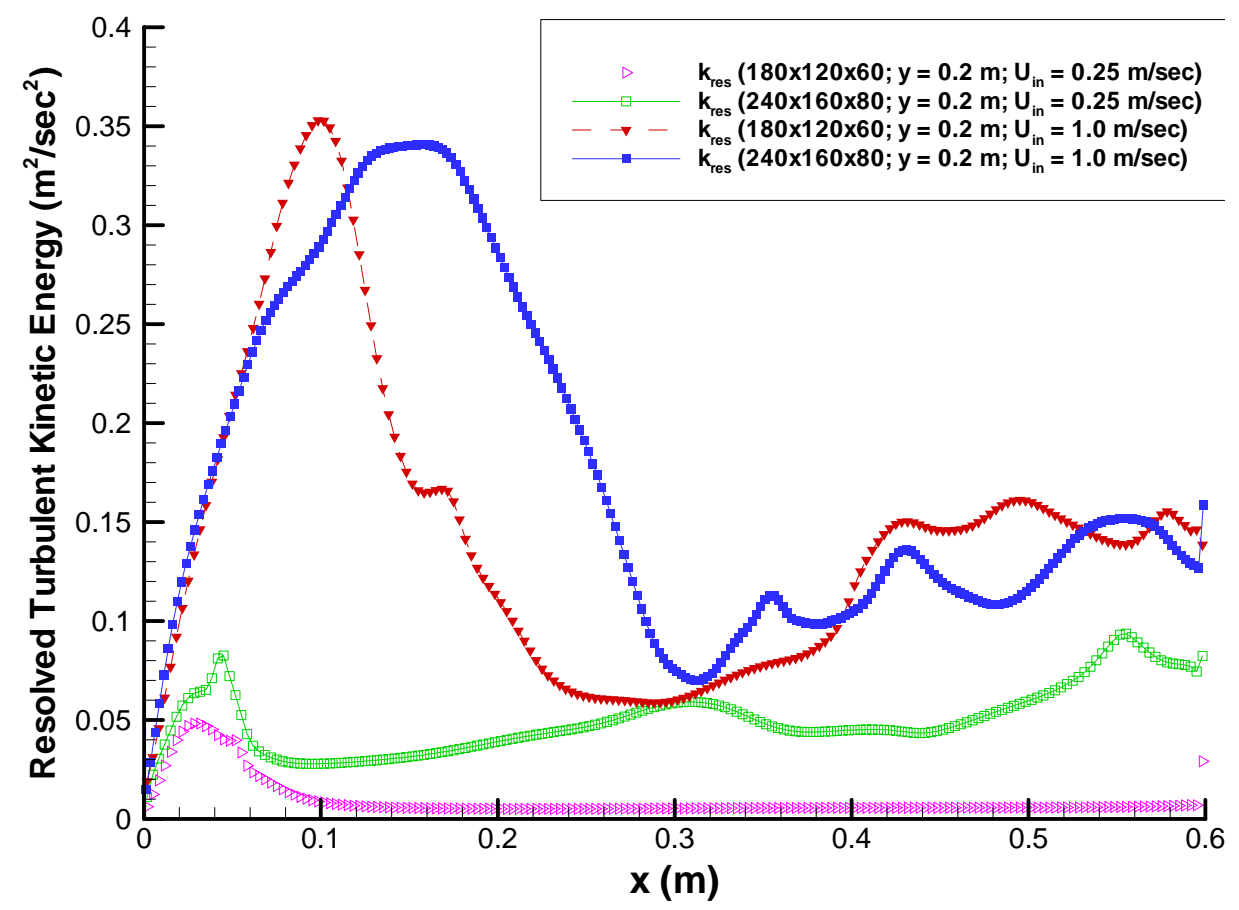

Figure 7.34 - Resolved turbulent kinetic energy at $y=0.20 \mathrm{~m}$ for $180 \times 120 \times 60$ and $240 \times 160 \times 80$ grids; $d t=0.001 ; U_{\text {inlet }}=0.25$ and $1.0 \mathrm{~m} / \mathrm{sec} ; \mathrm{Fr}=1.91$ and 7.65 .

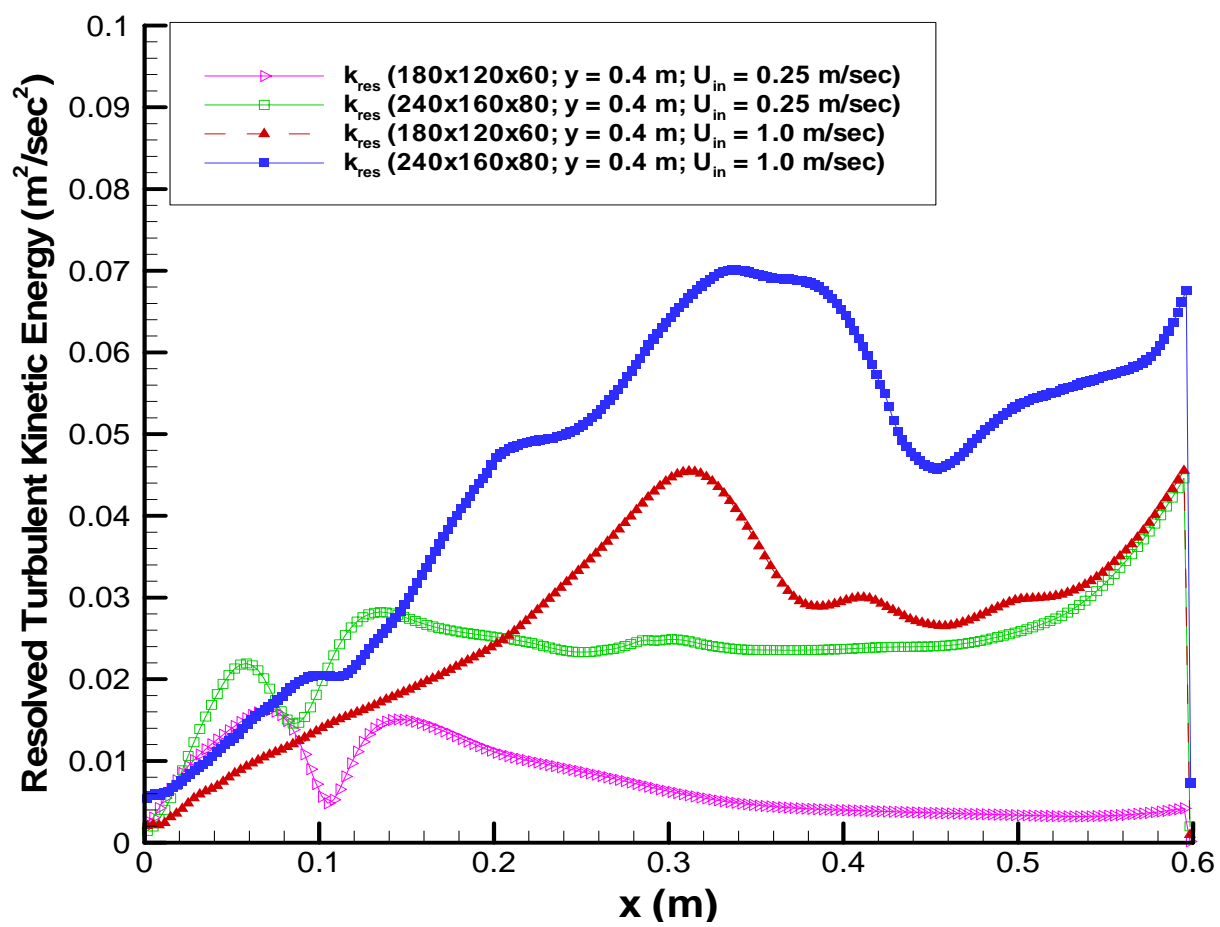

Figure 7.35 - Resolved turbulent kinetic energy at $y=0.40 \mathrm{~m}$ for $180 \times 120 \times 60$ and $240 \times 160 \times 80$ grids; $d t=0.001 ; U_{\text {inlet }}=0.25$ and $1.0 \mathrm{~m} / \mathrm{sec} ; \mathrm{Fr}=1.91$ and 7.65 


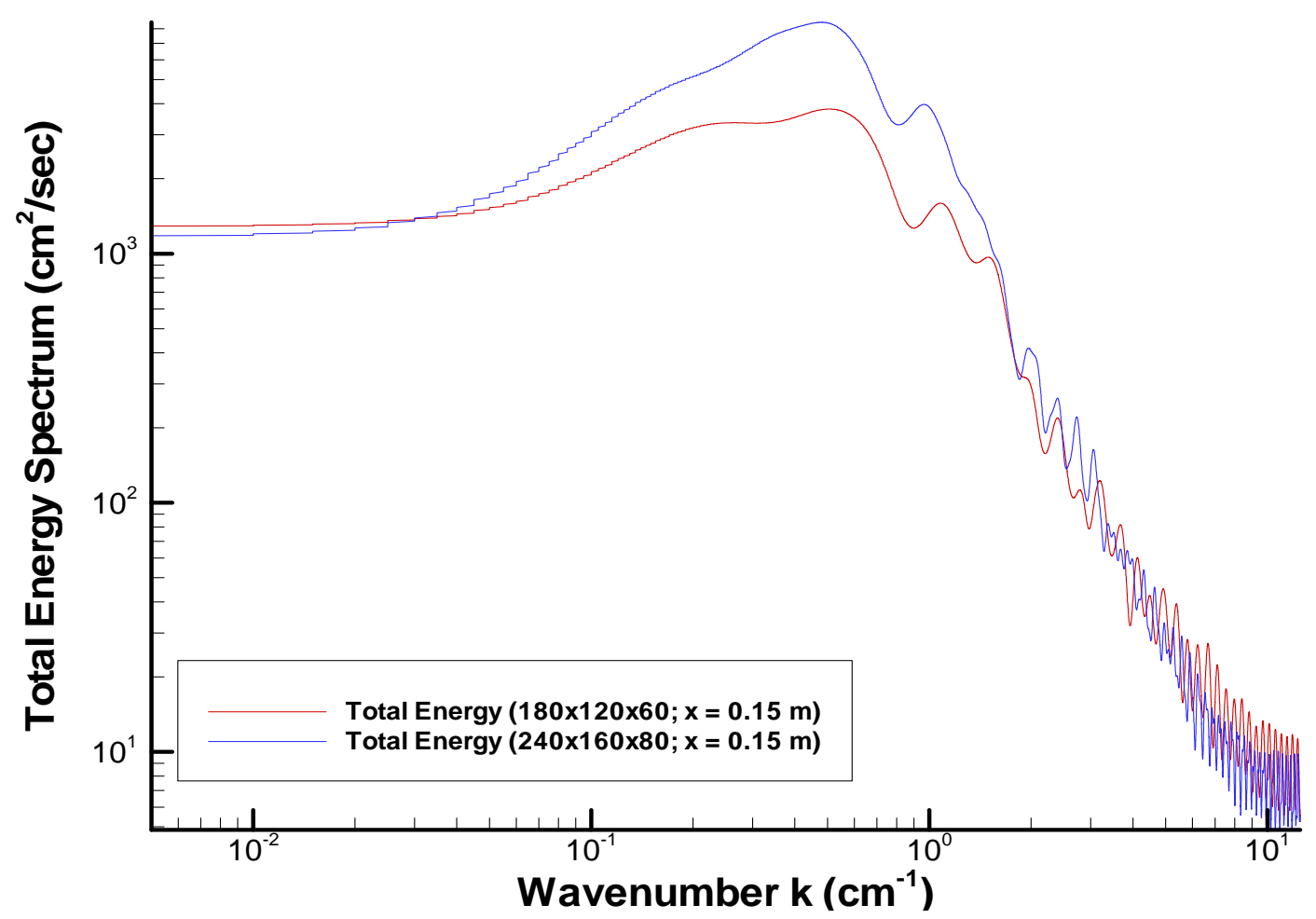

Figure 7.36 - Total averaged energy spectrum at $x=0.15 \mathrm{~m}$ for $180 \times 120 \times 60$ and $240 \times 160 \times 80$ grids; $d t=0.001 ; U_{\text {inlet }}=1.0 \mathrm{~m} / \mathrm{sec} ; \mathrm{Fr}=7.65$

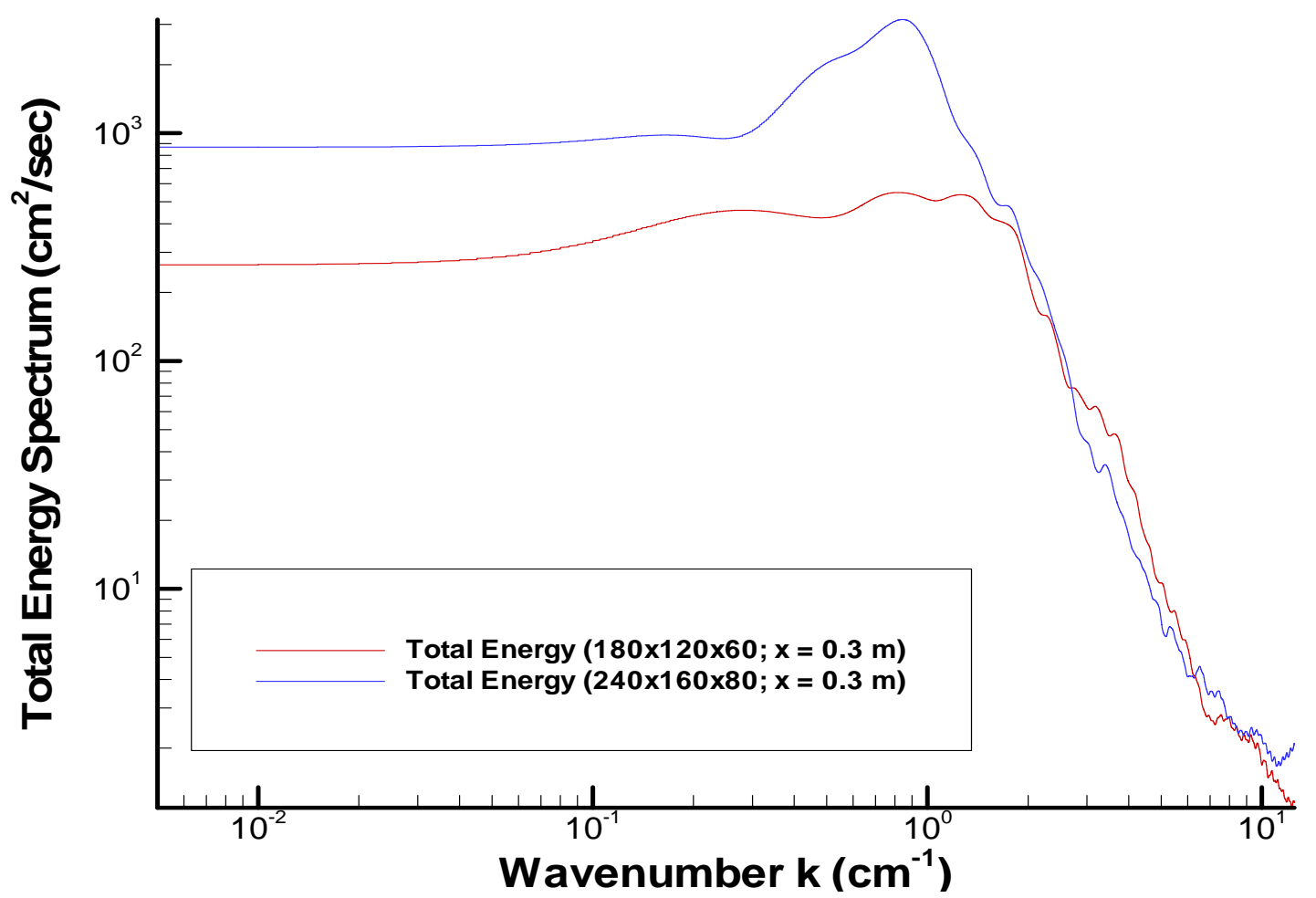

Figure 7.37 - Total averaged energy spectrum at $x=0.30 \mathrm{~m}$ for $180 \times 120 \times 60$ and $240 \times 160 \times 80$ grids; $d t=0.001 ; U_{\text {inlet }}=1.0 \mathrm{~m} / \mathrm{sec} ; \mathrm{Fr}=7.65$ 


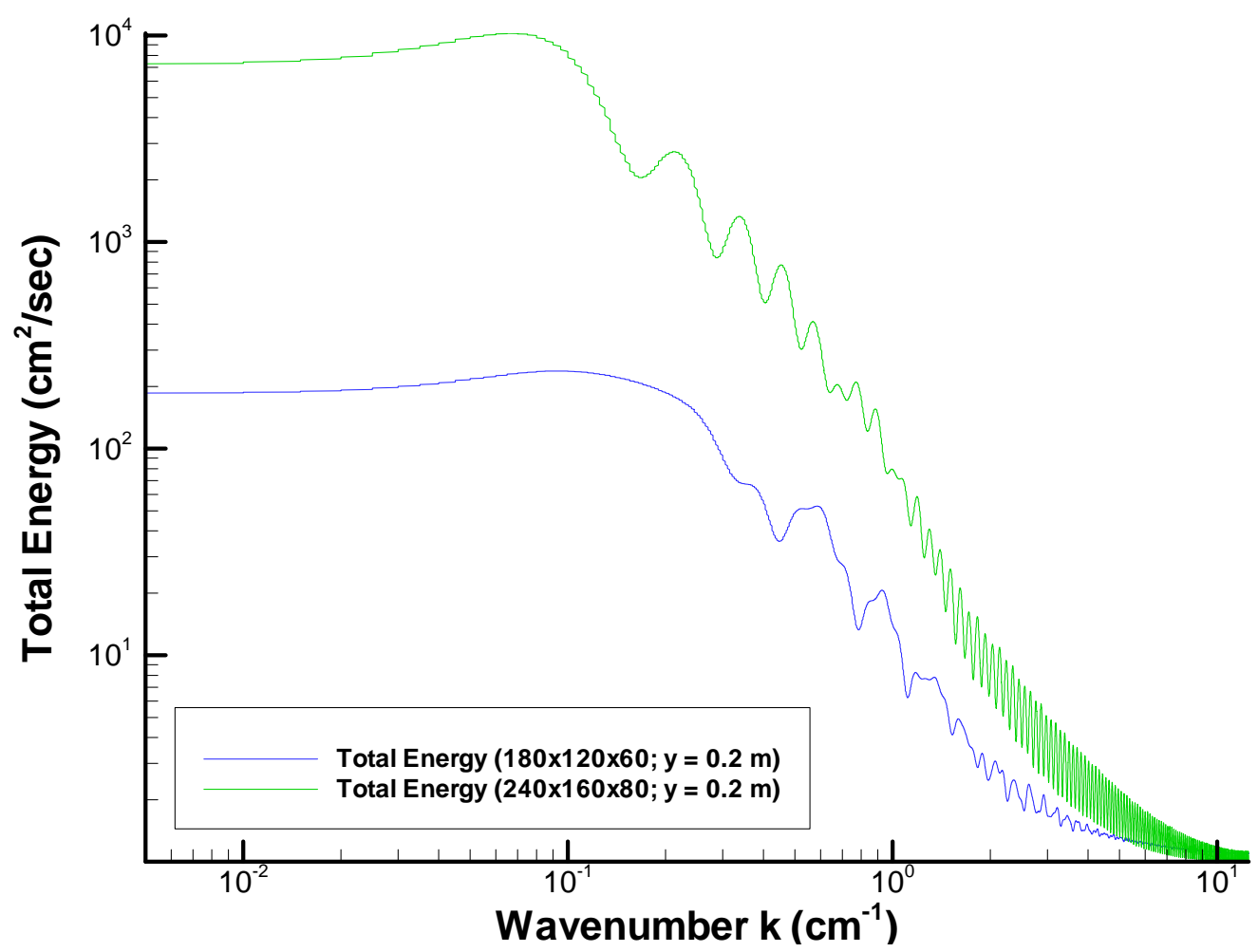

Figure 7.38 - Total averaged energy spectrum at $y=0.20 \mathrm{~m}$ for $180 \times 120 \times 60$ and $240 \times 160 \times 80$ grids; $d t=0.001 ; U_{\text {inlet }}=1.0 \mathrm{~m} / \mathrm{sec} ; \mathrm{Fr}=7.65$

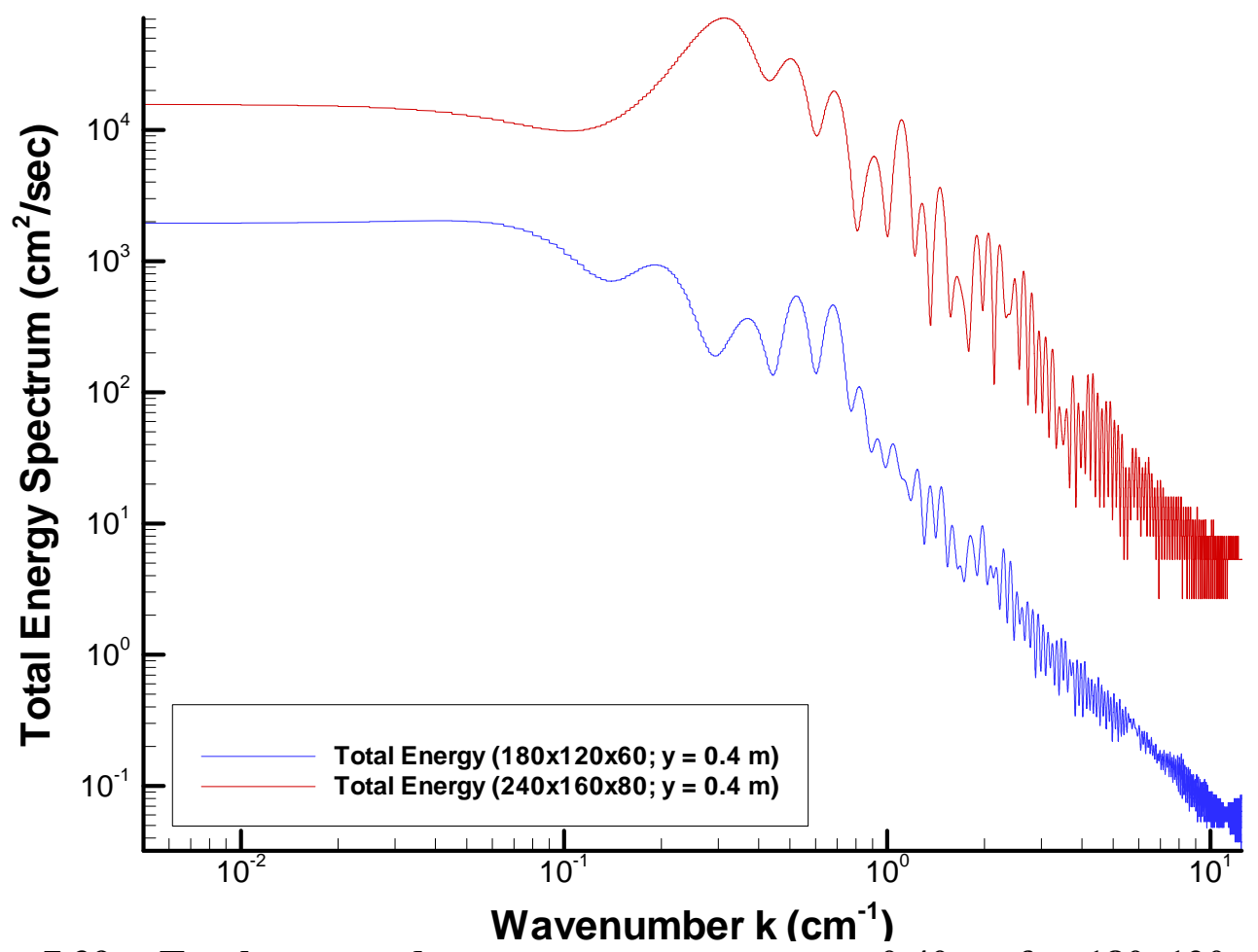

Figure 7.39 - Total averaged energy spectrum at $y=0.40 \mathrm{~m}$ for $180 \times 120 \times 60$ and $240 \times 160 \times 80$ grids; $d t=0.001 ; U_{\text {inlet }}=1.0 \mathrm{~m} / \mathrm{sec} ; \mathrm{Fr}=7.65$ 


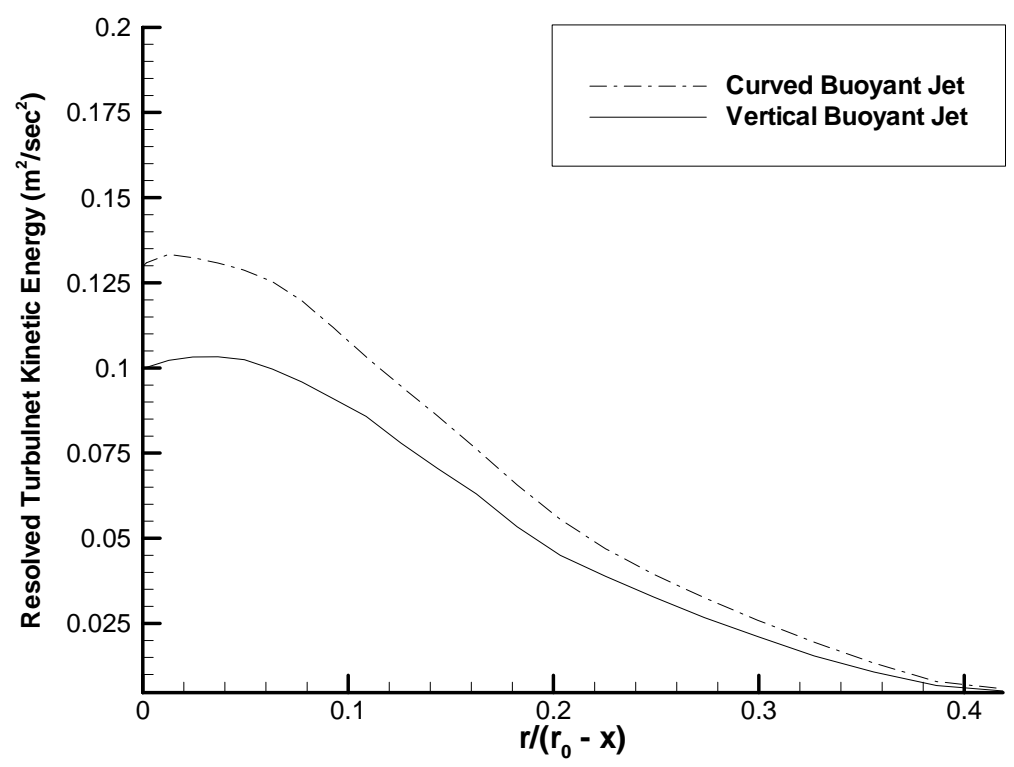

Figure 7.40 - Comparison of the resolved turbulent kinetic energy from the curved buoayant jet $\left(U_{\text {in }}=0.25 \mathrm{~m} / \mathrm{sec} ; \mathrm{Fr}=1.91\right)$ and vertical buoyant jet $\left(U_{\text {in }}=0.25 \mathrm{~m} / \mathrm{sec}\right.$; Fr $=2.8)$ DREAM ${ }^{\circledR}$ simulations. 


\subsection{CONCLUSIONS AND RECOMMENDATIONS}

\subsection{Conclusions}

As computer resources continue to develop, computational and numerical solution of complex turbulent flows becomes more feasible. In this journey, new numerical models will be developed, which will accurately solve complex flow phenomena within acceptable turn-around time. Although large eddy simulations (LES) have been around for many years, the ability to apply these methods to high-Reynolds number, complex geometries has been difficult at times because of the high demand on the computational resources. Application of a high degree of resolution, as well as subgrid-scale modeling further exacerbates the demands. To ensure the quality of the LES, efficient and accurate numerical methods must be applied. Recently new way of presentation of ITM methods has come around, whereby no subgrid-scale model is used and only the grid resolution, in cooperation with monotonic schemes or finite volumes, has been used to resolve the flow field. This type of large eddy simulation is known as implicit turbulence modeling (ITM), and has been utilized within this study.

The solution methodologies for the three-dimensional, unsteady, variable density, incompressible Navier-Stokes equations have been verified by applying them to four benchmarks: flow past a square cylinder, shear layer, impingement of a water jet onto a stably stratified fuel/water environment, and a vertical buoyant jet. Through comparison of the simulations from the DREAM ${ }^{\circledR}$ code for a variety of different grid sizes with the available experimental data, it has been shown that the solution methodology as implemented into DREAM ${ }^{\circledR}$ is capable of yielding reasonable results. Unfortunately, the efficiency of DREAM $^{\circledR}$ is limited by the time step size and grid resolution. A comparison of the ITM methods to Smagorinsky type subgrid-scale (SGS) models shows that in some simulations, with an appropriate flow field resolution, ITM methods outperform SGS models in the capturing of the physics of the flow field. However, for

under-resolved simulations, SGS models outperform LES ITM methods and may be more efficient if the time-step size and grid resolutions can be reduced using the SGS model. 
LES and ITM methods of turbulent flows are still in their infancy, as many scientists maintain the desire to use explicit subgrid-scale models. The use of ITM methods still requires a high degree of grid resolution to capture the physics of the flow field. For the simulations presented in this study, application of LES and ITM methods to the study of curved buoyant jets of oil in water within an enclosure has been performed, oil is injected at a specified flow rate into a water filled tank, initially taken to be stagnant. Grid resolution and time-step size has been investigated, and found to be highly important in ITM methods. Although the results appear to be too diffusive, much information from the simulations may still be determined. Buoyant forces cause the oil to rise as it flows downstream; the penetration distance is proportional to the initial momentum of the jet. The impingement of the oil onto the water results in a development of a plume, which later impinges on the vertical wall. Results show that even though the density stratification tends to damp the amount of turbulence present within the jet near the stably stratified interface, the ITM method as implemented into DREAM ${ }^{\circledR}$, seems capable of predicting a reasonable amount of turbulence. The resulting contours appear to be too dissipative, but they are statistically relevant and do predict some turbulence within the jet. The impingement of the jet on the walls results in a generation of turbulence within the enclosure. Analysis of the curved buoyant jet also shows that at an appropriate downstream location, similarity is achieved. Comparison between the curved buoyant jet simulations to the available experimental data shows good agreement for the centerline path of the jet based on the densimetric Froude number. Unfortunately, due to inadequate grid resolution, the jet spreading is under-predicted and the corresponding Reynolds stresses were not resolved at the liquid-liquid interface. It may be concluded that near the jet centerline, the turbulence quantities are resolved with some degree of accuracy, indicating that if a coarse grid is used for the simulations, as with those presented in this study, some empirical entrainment function may be necessary. A finer grid resolution run is currently under way. The preliminary results of this run will be published in a forth coming symposium paper.

The physics of the curved buoyant jet exhibits behavior much like the nonbuoyant and buoyant vertical jets by displaying similarity for some of the cases studied. Comparison to available experimental data correlates well with the simulations. 
Investigation of the turbulent characteristics, i.e. turbulence intensity and resolved turbulent kinetic energy flow field, may be deemed similar at best through this observation. Thus, it may be concluded that the application of these methods to immiscible fluids shows a new dimension to ITM and allows prediction of the turbulent flow field without the need for an explicit SGS model, albeit at a somewhat increased cost.

\subsection{Recommendations}

Accuracy and efficiency are the primary issues in large eddy simulations of high Reynolds number flows. In the past, most would suggest extension of the code to a higher order numerical scheme, such as fourth order, etc., since DREAM ${ }^{\circledR}$ is second order accurate in space and time. However, as ITM methods have been shown to accurately resolve flows with first order schemes, it is the grid resolution that needs to be investigated in conjunction with the possible use of higher-order schemes. It must be noted for future reference that doing so may make it difficult to ensure monotonicity and positivity, so this is where the future lies.

All of the simulations presented in this work were performed on a single, dual, or quad processor; however, no parallelization of the code was performed. The highest flow field resolution simulations ran for about one month to produce some meaningful results. The simulations used a grid resolution of approximately 50 to 100 times the Kolmogorov length scale. To truly investigate the behavior of ITM methods on variable density flows, it would be appropriate to try to reduce that resolution to 20 - 40 times the Kolmogorov length scale. Thus, parallel computation with a domain decomposition technique is desirable. Also, as a non-uniform grid was used in the vertical buoyant jet simulations and accurate results were obtained, it would seem reasonable to determine an appropriate non-uniform grid applicable to the curved buoyant jet flow. Investigation into how to relax the small time step requirement is also recommended.

Investigation of different types of interface tracking numerical schemes would also benefit future research. The scheme applied in this study utilizes only the grid 
resolution to track the liquid-liquid interface. A more appropriate method may be to use a conservative interface-reconstruction scheme, where the high resolution of the flow field would ensure capturing of more of the interface phenomena. Many different techniques are available; however, to use in conjunction with ITM methods, it is important to maintain the positivity and monotonicity of the scheme.

It may also be useful to implement the Droplet Formation Model, as described in Kandil (2001). A constant droplet size was utilized in the slip-velocity relationship, and previous studies have shown this was not adequate. The Droplet Formation Model would enhance the results shown in this study. Accounting for the surface tension near the water-fuel interface would clearly allow for this phenomenon to be better solved for higher densimetric Froude number flows. Implementation of an entrainment model would also allow the use of a coarser grid resolution in solving for the curved buoyant jet flows. 


\section{BIBLIOGRAPHY}

Amsden, A., O'Rourke, P., and James, T. (1989). "Three Dimensional Numerical Simulations of the UPS-292 Stratified Charge Engine." SAE Technical Paper Series: 18 - 24.

Anwar, H. (1969) "Experiment on an Effluent Discharging from a Slot into Stationary or Slow Moving Fluid of Greater Density." Journal of Hydraulic Research 7(4) 411 -430 .

Auerbach, D. (1991). "Entrainment and Ejection in Vortex Ring Flow." Zeitschrift fur Angewandte Mathematick und Mechanik 71: 506-507.

Baines, W. (1985). "Entrainment by a Buoyant Jet Flowing Along Vertical Walls." Journal of Hydraulic Research 23(3): 221 - 228.

Baines, W., Turner, J., and Campbell, I. (1990) "Turbulent Fountains in an Open Chamber." Journal of Fluid Mechanics 212: 557 - 592.

Boris, J. (1993). On Large Eddy Simulation using Sub-Grid Turbulence Models. Whither Turbulence? Turbulence at a Crossroads. New York City, Springer: 344 - 353.

Breuer, M. (1998). "Numerical and Modeling Influences on Large Eddy Simulations for the Flow Past a Circular Cylinder." The International Journal of Heat and Fluid Flow 19(5): 512 - 521.

Cavalletti, A., and Davies, P. (2003). Impact of Vertical, Turbulent, Planar, Negatively Buoyant Jet with Rigid Horizontal Bottom Boundary. Journal of Hydraulic Engineering. 129: 54 - 62.

Cehreli, Z. (2003). Personal Communication.

Chang, H. (1995). "Numerical Method of Unsteady Incompressible Navier-Stokes Equations for Simulation of Vortex Formation Around an Oscillating Body." American Society of Mechanical Engineers, Fluids Engineering Division (Publication) FED - Unsteady Flows 216: 97-102.

Cooper, L. (1989). "Heat Transfer in Compartment Fires Near Regions of Ceiling-Jet Impingement on a Wall." Journal of Heat Transfer, Transactions ASME 111(2): $455-460$.

Durbin, P., and Petterson Reif, B. (2001). Statistical Theory and Modeling for Turbulent Flows. West Sussex, England, John Wiley and Sons, Ltd.

Friedman, P. D., and Katz, J. (1999). "Flow and Mixing Mechanisms Caused by the Impingement of an Immiscible Interface with a Vertical Jet." Physics of Fluids 11(9): 2598 - 2612.

Friedman, P. D., Winthrop, A.L., and Katz, J. (2001). "Droplet Formation and Size Distributions from an Immiscible Interface Impinged with a Vertical Negatively Buoyant Jet." Atomization and Sprays 11: 269 - 290.

Fureby, C. (1999). "Large Eddy Simulation of Rearward Facing Step." AIAA Journal 37(11): 1401 - 1410.

Fureby, C., and Grinstein, F. (1999). "Monotonically Integrated Large Eddy Simulation of Free Shear Flows." AIAA Journal 37(5): 544 - 556.

Gel, A. (1999). A Distributed-Memory Implementation of KIVA-3 with Refinements for Large Eddy SImulation. Mechanical and Aerospace Engineering. Morgantown, West Virginia University: 259.

George, W. and Tamanini F. (1977) “Turbulence Measurements in an Axisymmetric 
Buoyant Plume." Int. J. Heat and Mass Trans. 20 : 1145 - 1154.

Gu, R. (1998). "Numerical Simulation of 2D Buoyant Jets in Ice-Covered and Temperature Stratified Water." Advances in Water Resources 21(5): 363 - 373.

Harten, A. (1983). "Nonoscillatory Second-Order Accurate Shock Capturing Schemes." Pluralis: 35-43.

Hu, G. (2002). Uncertainty Assessment For CFD Using Error Transport Equation. Mechanical and Aerospace Engineering. Morgantown, West Virginia, West Virginia University.

Hussein, H. J., Capp, S., and George, W. (1994). "Velocity Measurements in a HighReynolds Number, Momentum-Conserving, Axisymmetric, Turbulent Jet." Journal of Fluid Mechanics 258: 31-75.

Hwang, R., and Chiang, T. (1995). "Numerical Simulation of Vertical Forced Plume in a Crossflow of Stably Stratified Fluid." Journal of Fluids Engineering 117: 696 705.

Kandil, S. (2001). A Computational Study of Mixing in a Liquid Jet Impinging on an Immiscible Liquid Layer. Mechanical and Aerospace Engineering. Morgantown, West Virginia, West Virginia University.

Kang, S., and Greif, R. (1992). "Flow and Heat Transfer to a Circular Cylinder with a Hot Impinging Air Jet." International Journal of Heat and Mass Transfer 35(9): 2173 - 2183.

Knight, D., Zhou, G., Okong'o, N., and Shukla, V. (1998). "Compressible Large Eddy Simulation using Unstructured Grids." AIAA Journal 98: 522 - 539.

List, E. (1982). "Turbulent Jets and Plumes." Annual Review of Fluid Mechanics 14: $189-212$.

Lyn, D., Einav, S., Rodi, W., and Park, J. (1995). "A Laser Doppler Velocimetry Study of Ensemble-Averaged Characteristics of the Turbulent Near Wake of a Square Cylinder." Journal of Fluid Mechanics 304: 285 - 319.

Lyn, D., Einav, S., Rodi, W., and Park, J. (1995). "A Laser-Doppler Velocimetry Study of Ensemble-Averaged Characteristics of the Turbulent Wake of a Square Cylinder." Journal of Fluid Mechanics 304: 285 - 319.

Margolin, L., and Rider, W. (2001). "A Rationale for Implicit Turbulence Modeling." International Journal for Numerical Methods in Fluids 39: 821 - 841.

Margolin, L., Smolarkiewicz, P., and Wyszogrodzki, A. (2002). "Implicit Turbulence Modeling for High Reynolds Number Flows." Transactions of the ASME 124(December): 862 - 867.

Mathew, J., and Basu, A. (1994). The Mechanism of Entrainment in Circular Jets. Fourteenth International Conference on Numerical Methods in Fluids, Bangalore, India.

Mathworks Incoporated (2002). Image Processing Toolbox. Natick, Massachusetts, The Mathworks, Inc.

Noutsopoulos, G., Demetriou, J., Gini, M., and Stamou, A. (1979). "Flow Geometry of a Vertical Buoyant Jet Striking a Concentric Disc." Scientific Papers 3(3): 1 - 12.

Okong'o, N., and Knight, D. (1998). "Compressible Large Eddy Simulation using 
Unstructured Grids: Channel and Boundary Layer Flows." AIAA Journal 98(3315): 623 - 628.

Oran, E., and Boris, J. (1993). "Computing Turbulent Shear Flows - A Convenient

Conspiracy." Computers in Fluids 7(5): 523 - 533.

Patankar, S. (1980). Numerical Heat Transfer and Fluid Flow. New York City, Hemisphere Publishing.

Pope, S. (2000). Turbulent Flows. Cambridge, UK, Cambridge University Press.

Porter, D., Pouquet, A., and Woodward, P. (1994). "Kolmogorov-like Spectra in Decaying Three-Dimensional Supersonic Flows." Physics of Fluids 6(6): 2133 2142.

Rai, M., and Moin, P. (1991). "Direct Simulation of Turbulent Flows using Finite Difference Schemes.” Journal of Computational Physics. 109: 169 - 182.

Railston, W. (1954) "The Decay Law of a Naturally Convected Air Stream." Proceedings of the Physical Society. 67B: $42-51$.

Razinsky, E. and Brighton, J. (1971) "Confined Jet Mixing for Non-Separating Conditions." Trans. Of the A.S.M.E. Journal of Basic Engineering. 333 - 349.

Rightley, P. (1995). Bubble Dispersion and Interphase Coupling in a Free Shear Flow. Mechanical Engineering. San Diego, California, University of California.

Rodi, W. (1997). Large Eddy Simulation of the Flow Past Bluff Bodies: State of the Art. Proceedings from the International Conference of Fluid Engineering; JSME Centennial Grand Congress, Tokyo, Japan.

Sangras, R., Dai, Z., and Faeth, G. (2000). "Velocity Statistics of Plane Self-Preserving Buoyant Turbulent Adiabatic Wall Plumes." Journal of Heat Transfer 122: 693 700.

Shi, S. (2001). Large Eddy Simulation of Ship Wakes. Mechanical and Aerospace Engineering. Morgantown, West Virginia, West Virginia University.

Shy, S. (1995). "Mixing Dynamics of Jet Interaction with a Sharp Density Interface." Experimental Thermal and Fluid Science 10(3): 355 - 340.

Smith, J. (1996). An Accurate Navier-Stokes Solver with an Application to Unsteady Flows. Mechanical and Aerospace Engineering. Morgantown, West Virginia University: 413.

Smolarkiewicz, P., and Margolin, L. (1998). "MPDATA: A Finite-Difference Solver for Geophysical Flows." Journal of Computational Physics 140: 459 - 480.

Sohankar, A., Davidson, L., and Norberg, C. (2000). "Large Eddy Simulation of Flow Past a Square Cylinder: Comparison of Different Subgrid-scale Models." Journal of Fluids Engineering 122: 39 - 52.

Stone, C., and Kwan, E. (1969). "Simultaneous Solution of Multiphase Reservoir Flow Equations." Society of Petroleum Engineering 10(2): 99 - 110.

Tamura, T., and Kuwahara, K. (1989). "Numerical Analysis on Aerodynamic Characterstics of an Inclined Square Cylinder." AIAA Journal 89(1805): 129 137.

Tennekes, H., and Lumley, J. (1999). A First Course in Turbulence. Cambridge, Massachusetts, The MIT Press.

Turner, J.S. (1986) "Turbulent Entrainment: The Development of the Entrainment 
Assumption, and its Application to Geophysical Flows." Journal of Fluid Mechanics 173: 431 - 471.

Urbin, G., and Knight, D. (2001). "Large Eddy Simulation of Supersonic Boundary Layer Using an Unstructured Grid." AIAA Journal 39(7): 1288 - 1295.

Vreman, B., Geurts, B., and Kuerten, H. (1997). "Large Eddy Simulation of the Turbulent Mixing Layer." Journal of Fluid Mechanics 339: 357 - 390.

Yee, H. C., and Harten, A. (1987). "Implicit TVD Schemes for Hyperbolic Conservation Laws in Curvilinear Coordinates." AIAA Journal 25(2): 266-274.

Yee, H. C., Harten, A., and Yuan, T. (1985). "Implicit TVD Schemes for Hyperbolic Conservation Laws in Curvilinear Coordinates." AIAA Journal: 228 - 241.

Yee, H. C., Harten, A., and Yuan, T. (1986). "Linearized form of Implicit TVD Schemes for the Multidimensional Euler and Navier-Stokes Equations." Computers and Mathematics with Applications 12A(4-5): 413 - 432.

Yi, T., and Baldacchino, F. (1995). "Computer Simulation of Diffusion and Adsorption in Pillared Clays." Materials Research Society Symposium - Proceedings Synthesis and Properties of Advanced Catalytic Materials 368: 357 - 362.

Zhou, X., Luo, K., and Williams, J. (2001). "Large-Eddy Simulation of Turbulent Forced Plume." European Journal of Mechanics B - Fluids 20: 233 - 254. 


\section{APPENDIX A - VERIFICATION OF SCALAR SOLVER}

\section{A.1 Introduction}

This section, although not explicitly part of the overall goal of this study, is important because it introduces and verifies the Quasi-Second Order Upwinding (QSOU) scheme implemented into DREAM ${ }^{\circledR}$ and used for the determination of the variable density flows. Thus, although intended to be brief in nature, a literature review of the scheme is presented, as well as an alternative method to solving for the density interface in multiphase flows. This section will end with a small study showing that after a variable density scheme is implemented, that it satisfies the conservation of mass.

\section{A.2 Literature Review of QSOU}

The difficulty in Computational Fluid Dynamics arises when trying to accurately solve for the convection of some scalar quantity, i.e. concentration, volume fraction, etc., as the accuracy is highly coupled with the numerical scheme. For accuracy, a second order scheme is generally required, as this order tends to limit the amount of numerical diffusion present in the calculations. One should never achieve negative scalar values, i.e. negative quantities of fuel in water, and proper steps need to be taken to ensure this. First order schemes, and certain hybrid schemes, are mostly used to prevent "wiggles" introduced through using central differencing schemes when the local cell Peclet number is larger than a certain value. However, these schemes do in fact introduce dissipation of the numerical code, which may in fact exceed physical limits. A drawback to using a second order scheme arises in the computational cost, as first order schemes run faster. This section focuses on the second order numerical scheme used in DREAM ${ }^{\circledR}$ to solve the generic scalar transport equation, which is the quasi-second order upwinding scheme, with a short literature review of TVD, total variation diminishing, schemes at the end of this section. These two numerical schemes are reviewed simply because as mentioned previously, application of some second order schemes leads to wiggles; however, the Quasi-second order scheme, as well as the TVD scheme, exhibit a strong monotone 
property, which eliminates these wiggles from the solution, eliminating the need to using a larger number of grid nodes to refine and eliminate the wiggles (Yi and Baldacchino, 1995).

The quasi-second order upwind scheme, QSOU, is a numerical method used to solve the convection of a quantity based on the local flow direction, which then utilizes either a forward or backward differencing scheme to discretize the convection terms in the Navier-Stokes equation. Generally speaking, the QSOU scheme utilizes the minimum gradient at three cell locations, then adds this term to the convection based on the flow direction. It is important to note that negative scalar values must be eliminated and prevented by ensuring that cells can't flux more scalar than they have, and it can't accept more scalar than it has space for. This is one problem with the second order scheme used in Flow3D, as the cell may accept more scalar that is has room for, leading to negative values; however, a limiting routine does in fact solve this discrepancy. The general formulation to solve the scalar transport equation in DREAM $^{\circledR}$ is given below:

The general, three-dimensional scalar transport equation is given as

$$
\frac{\partial(\rho \phi)}{\partial t}+\frac{\partial}{\partial x_{j}}\left(\rho u_{j} \phi\right)=\frac{\partial}{\partial x_{j}}\left(\Gamma \frac{\partial \phi}{\partial x_{j}}\right)
$$

where $\Gamma$ represents the diffusion, and it is zero in only pure convection cases, which are not studied in this dissertation, but is used to verify this method in Section A.4. However, as QSOU is only applied to the convection terms, the diffusion component of Eq. (A.2.1) may be dropped for derivation purposes only, thus the discretization of the convection term in three dimensions is developed through integration of Eq. (A.2.1), which gives

$$
\frac{1}{\Delta t}\left[\left(\rho \phi_{P}\right)-\left(\rho \phi_{P}\right)^{o}\right] \Delta x \Delta y \Delta z=\left\{\begin{array}{l}
{\left[(\rho \mathrm{d} \phi)_{e}-(\rho \phi P)_{w}\right] \Delta y \Delta z+} \\
{\left[(\rho \mathrm{g} \phi)_{n}-(\rho \phi P)_{s}\right] \Delta x \Delta z+} \\
{\left[(\rho \mathrm{g} \phi)_{t}-(\rho \phi P)_{b}\right] \Delta x \Delta y}
\end{array}\right\}
$$


where the superscript represents the old time level and the subscript P represents cell centers. Thus, when applying this type of formulation to the east cell, the gradient at the east cell face is given as

$$
\left(\frac{\partial \phi}{\partial x}\right)_{e}=\operatorname{sign}\left(\phi_{E}-\phi_{P}\right) \min \left(\left|\frac{\phi_{E}-\phi_{P}}{\Delta x_{e}}\right|,\left|\frac{\phi_{P}-\phi_{W}}{\Delta x_{e}}\right|\right)
$$

which may be applied in all directions. Then, Eq. (A.2.3) is added or subtracted to the east cell face, yielding

$$
\phi_{e}= \begin{cases}\phi_{E}-\left(\frac{\partial \phi}{\partial x}\right)_{e}\left|\frac{\Delta x_{e}}{2}\right| & \mathrm{u}<0 \\ \phi_{P}+\left(\frac{\partial \phi}{\partial x}\right)_{P}\left|\frac{\Delta x_{e}}{2}\right| & \mathrm{u}>0\end{cases}
$$

The discretized equation becomes

$$
a_{P} \phi_{P}=a_{E} \phi_{E}+a_{W} \phi_{W}+a_{N} \phi_{N}+a_{S} \phi_{S}+a_{T} \phi_{T}+a_{B} \phi_{B}
$$

Substitution of Eqs. (A.2.3) and (A.2.4) into Eq. (A.2.5) yields

$$
\phi_{P}=\frac{\sum a_{n b} \phi_{n b}}{a_{P}}
$$

where $n_{b}$ denotes neighboring cells (Cehreli, (2003) and Amsden et. al. (1989)). It is important to note that there are a variety of ways to solve these equations, and three solvers are available in DREAM ${ }^{\circledR}$, being the Stone Implicit three dimensional solver, an incomplete conjugate gradient solver, and a conjugate gradient stabilized solver.

The conclusion of this section deals with another family of upwinding secondorder accurate schemes being TVD (total variation diminishing) schemes. As with QSOU schemes, TVD schemes are strongly monotonic as no spurious oscillations are 
generated. The notion of TVD schemes was first introduced by Harten (1983) and there are many variations of the schemes. Some of these methods can also be viewed as threepoint central differencing schemes with a "smart" numerical diffusion mechanism. The term "smart" means that there is an automatic feedback mechanism to control the amount of numerical dissipation for nonlinear problems. In general, TVD schemes can be divided into two categories, being upwind or symmetric TVD schemes. A way of distinguishing an upwind from a symmetric TVD scheme is that the numerical dissipation term corresponding to an upwind TVD scheme is upwind weighted as opposed to the numerical dissipation term corresponding to a symmetric TVD scheme that is cell centered (Yee and Harten, 1987).

Harten's TVD scheme is derived by starting with a first-order TVD scheme and applying it to a modified flux, which is chosen so that the scheme is second order at regions of smoothness and first order at points of extrema. Although the scheme is an upwind scheme, it is written in a symmetric form, i.e. central difference plus an appropriate numerical dissipation term. For numerical derivation of a variety of TVD schemes, the reader is referred to Yee et. al. (1985) and Yee et. al. (1986). This formulation of a generic scalar solver is not used currently in DREAM ${ }^{\circledR}$; however, it may be an option to include a TVD scheme in the future. 


\section{A.3 Verification of QSOU}

Validation of QSOU as implemented into DREAM ${ }^{\circledR}$ is performed through comparison of simulations performed by $\mathrm{Hu}$ (2002); where a fairly general Fortran program using finite volume method was written to simulate a time dependent 3D scalar transport subject to an arbitrary fixed flow field. The transport phenomenon is governed by both convection and diffusion, and no extra sources are involved. The DREAM ${ }^{\circledR}$ code was modified to match the code by $\mathrm{Hu}$ (2002) exactly, including all boundary conditions. A thin square plate of dimensions $20 \mathrm{~cm}$ x $20 \mathrm{~cm}$ was used as the computational domain, which enables an assumption of zero velocity in z-direction for Hu's (2002) simulations, i.e., if the domain is divided in different layers along z-direction, nothing changes in that direction. The DREAM ${ }^{\circledR}$ simulations are on the $\mathrm{x}-\mathrm{z}$ plane; however, there should be no difference in the results. At boundaries of each $\mathrm{x}-\mathrm{y}$ plane $\phi$ is set to be zero for $\mathrm{Hu}$ (2002), whereas $\phi$ is set to be zero at the boundaries of the $\mathrm{x}-\mathrm{z}$ planes in $\operatorname{DREAM}^{\circledR}$; At top and bottom boundaries zero derivatives are defined.

With a square domain being used, the imposed flow field can be arbitrary. For example, it can be an uniform flow in a horizontal, vertical or diagonal direction, or a rotational flow field with constant angular velocity (vortex flow). This section initially compares results from DREAM ${ }^{\circledR}$ and $\mathrm{Hu}$ (2002) for a horizontal, vertical, diagonal, and then finally a rotational flow field. Fully explicit schemes are used in the simulations, and the diffusion is set to be zero, meaning only numerical diffusion is present. This is the only way a comparison between the two codes could be made. The mesh size used in both simulations was $40 \mathrm{x} 40$ in the $\mathrm{x}$ and $\mathrm{y}$ directions, respectively, and the scalar was initialized to have a value of 1.0. The domain used by $\mathrm{Hu}$ (2002) went from -10 to 10 in both directions, where as those used by DREAM ${ }^{\circledR}$ used a 0 to 20 domain. Again, the grid ratios and sizes were identical. Also, as an additional test of the dimensionality of DREAM $^{\circledR}$, the flow field is on the $\mathrm{x}-\mathrm{z}$ plane

For the flows used in the verification of QSOU as implemented in DREAM ${ }^{\circledR}$, have been verified by $\mathrm{Hu}$ (2002) in a comparison to the analytical solution, when 
available as there is no analytical solution available for the rotational flow field. Therefore, the reader is referred to $\mathrm{Hu}$ (2002) for further details on the verification of the flow fields and only the scalar results are compared to the DREAM ${ }^{\circledR}$ simulations. This section has the sole purpose of determining the QSOU numerical scheme has been implemented correctly into DREAM ${ }^{\circledR}$. The reader is referred to Section 2.6 for a derivation of the quasi-second order upwinding scalar transport scheme.

\section{Axial Flow Field}

Letting $u=1$ and $v=0$, the flow field becomes a one-dimensional convection and two-dimensional diffusion problem. Remember that the diffusion is only numerical diffusion. After running the program for a physical time of $3 \mathrm{~s}$ with implicitness factor equaling zero (fully explicit), the results of the transport of a generic scalar quantity in a uniform horizontal flow field are shown in Figures (A.3.1), simulations from $\mathrm{Hu}$ (2002) and (A.3.2), which shows the corresponding simulations from DREAM ${ }^{\circledR}$. It is evident from comparison of Figures (A.3.1) and (A.3.2), that the DREAM ${ }^{\circledR}$ simulations perform well for these types of flows. Numerical values differ in the $10^{\wedge}-3$ digit.

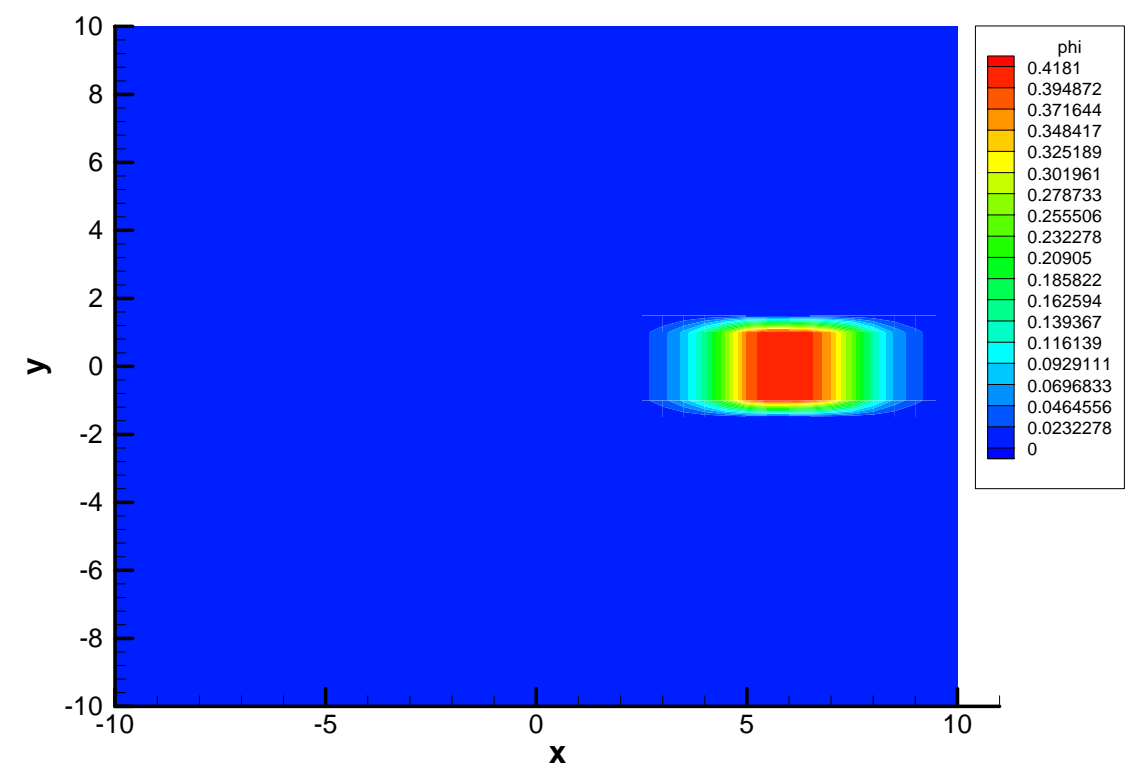

Figure A.3.1 - Results from Hu (2002) code for axial flow (40x40x5) after 10 seconds; pure convection; fully explicit 


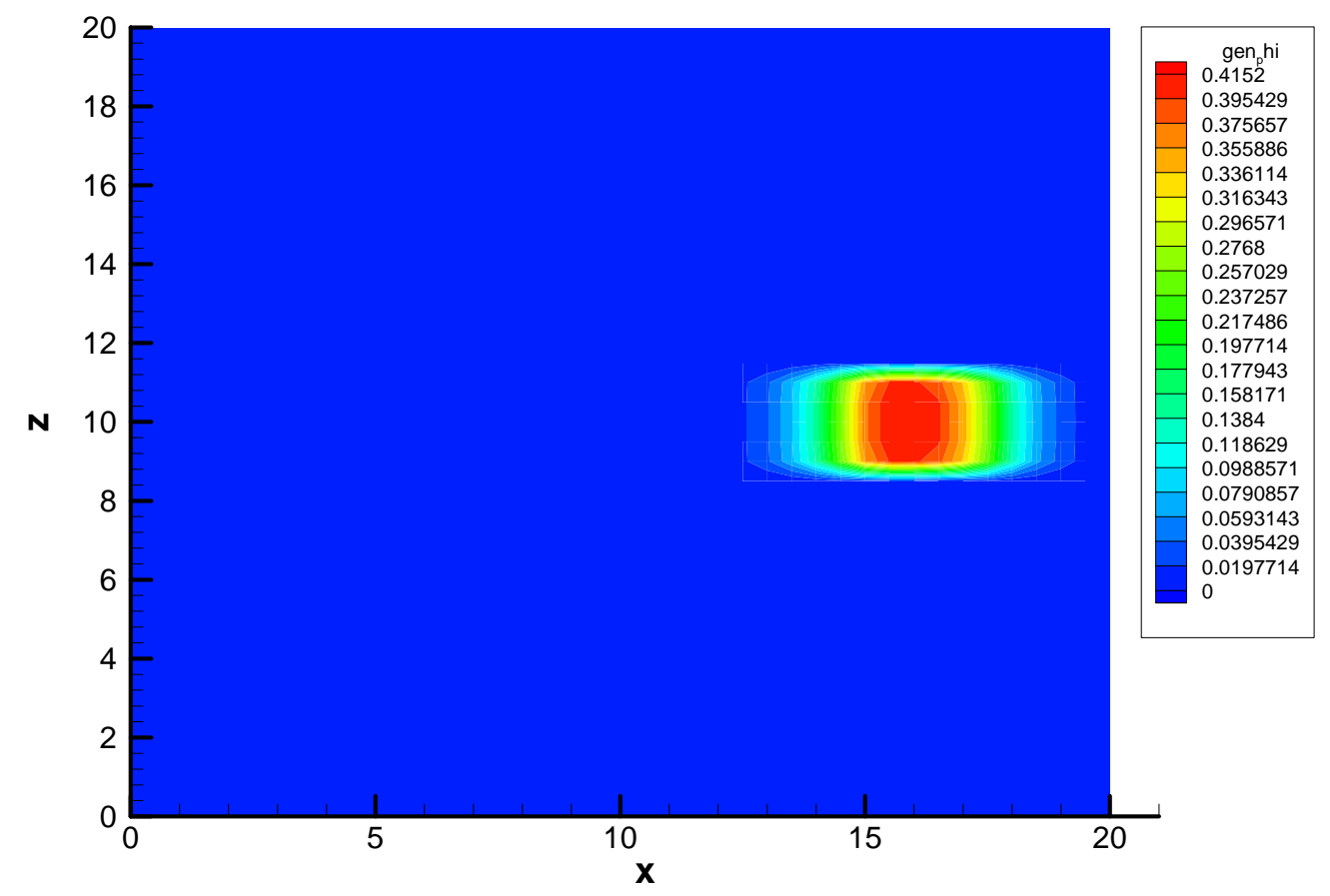

Figure A.3.2 - Results from DREAM ${ }^{\circledR}(40 \times 5 \times 40)$ after 10 seconds; pure convection; fully explicit

\section{Vertical Flow Field}

As a second code testing case, we impose a vertical flow field in the computational domain and relocate the initial pulse from $(-4,0)$ to $(0,-4)$. Other conditions remain the same. In this test, we check whether the same results but in a different direction can be obtained as those from the horizontal flow situation. Results from the simulations are shown in Figures (A.3.3) and (A.3.4), and again, excellent agreement is achieved when comparing the two simulations. 


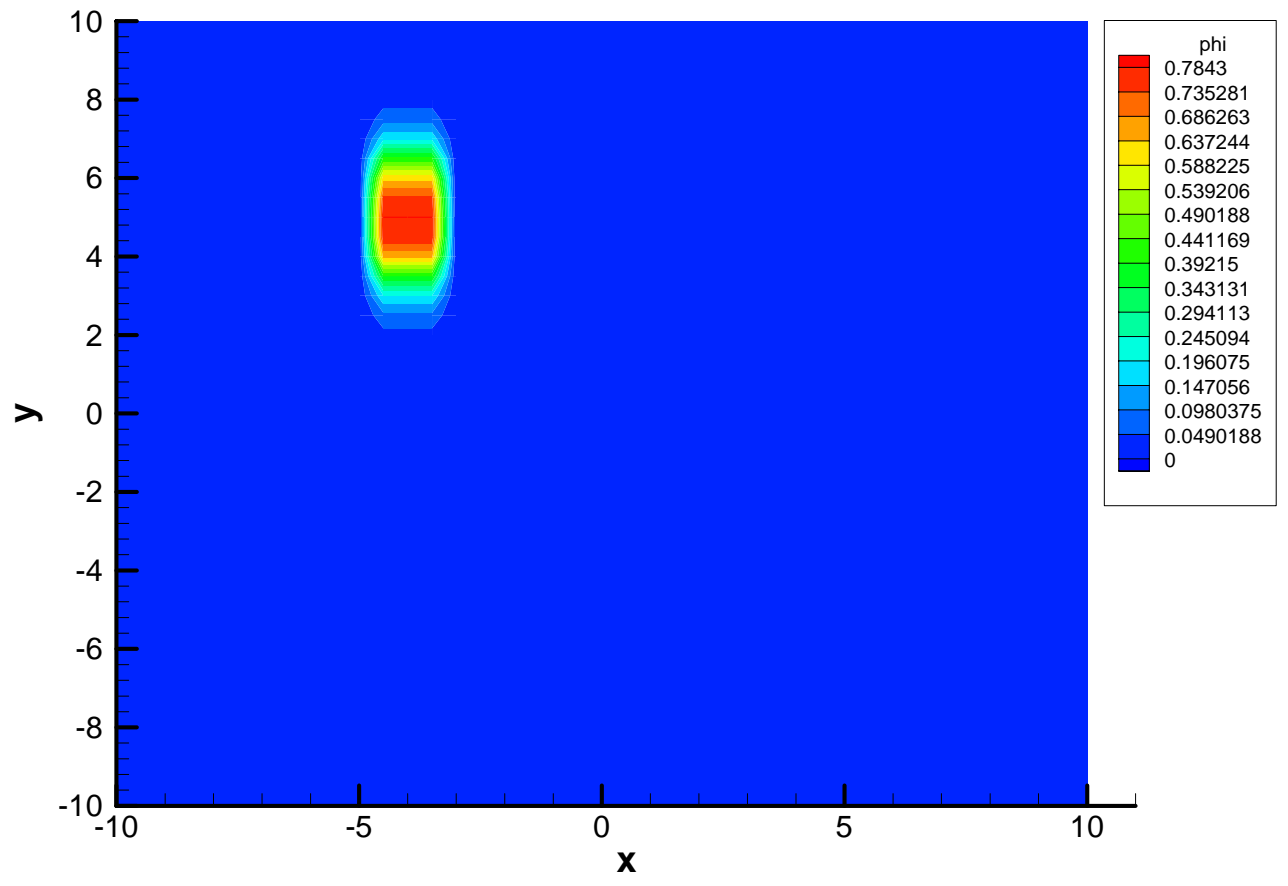

Figure A.3.3 - Transport of scalar for vertical flow using QSOU3D from Hu (2002)

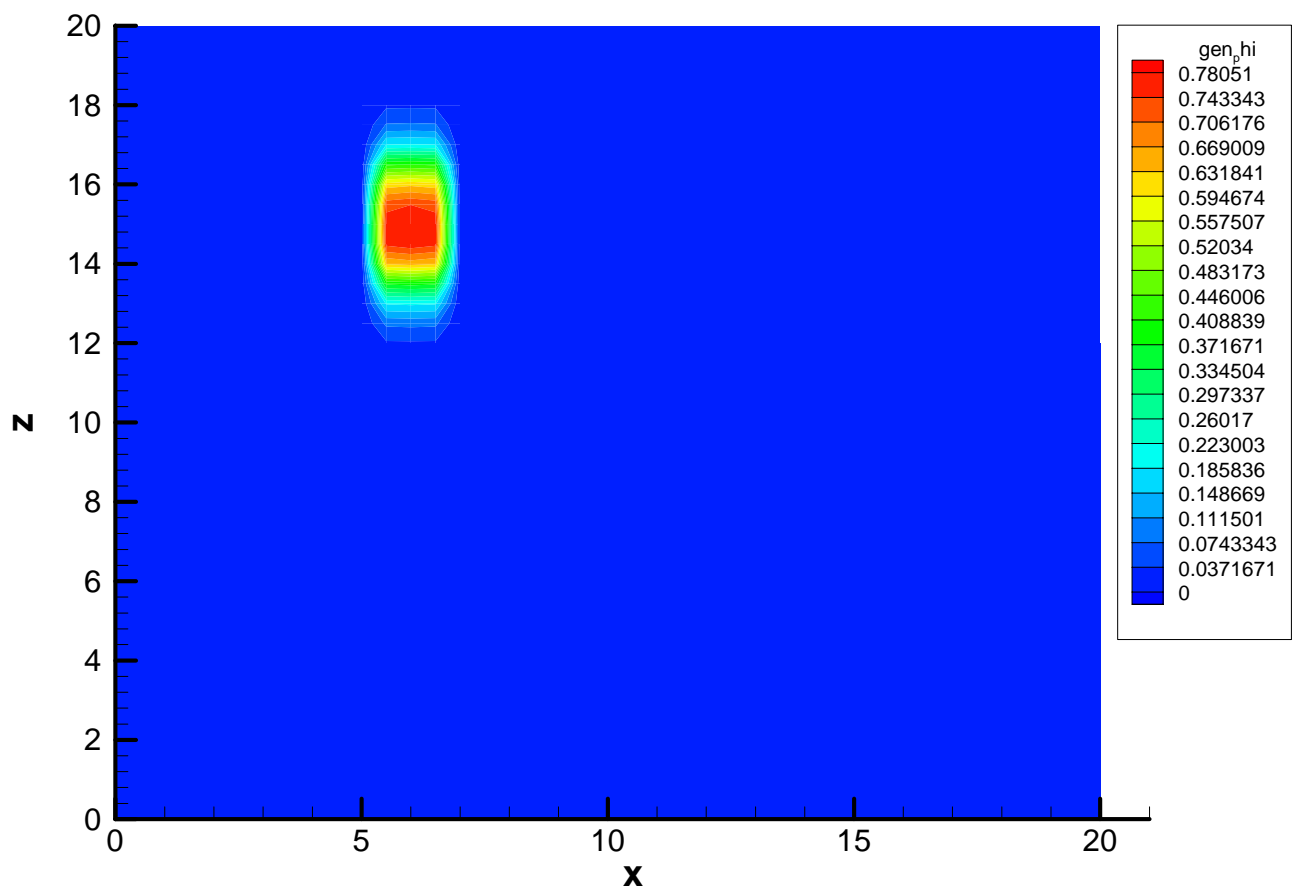

Figure A.3.4 - Results from transport of scalar quantity using QSOU3D in DREAM $^{\circledR}$ 


\section{Diagonal Flow Field}

Next, we proceed with similar testing steps for a constant diagonal flow field in which $u=1$ and $v=1$. Again, all other parameters are identical and the results of the simulations after 10 seconds are shown in Figures (A.3.5) and (A.3.6). Comparison of figures (A.3.5) and (A.3.6) show similar results for the diagonal flow field.

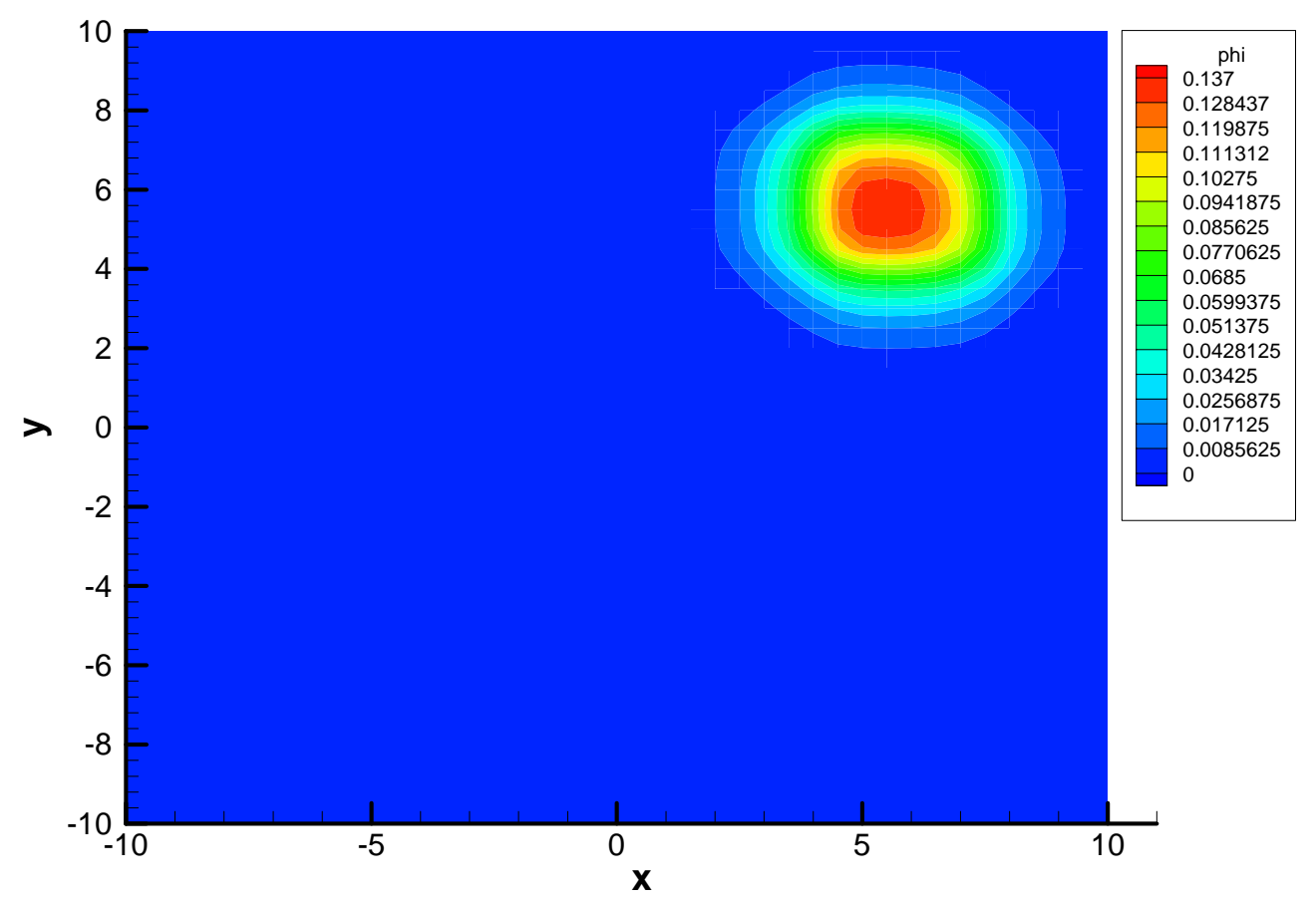

Figure A.3.5 - Results from transport of scalar quantity using Hu (2002) 


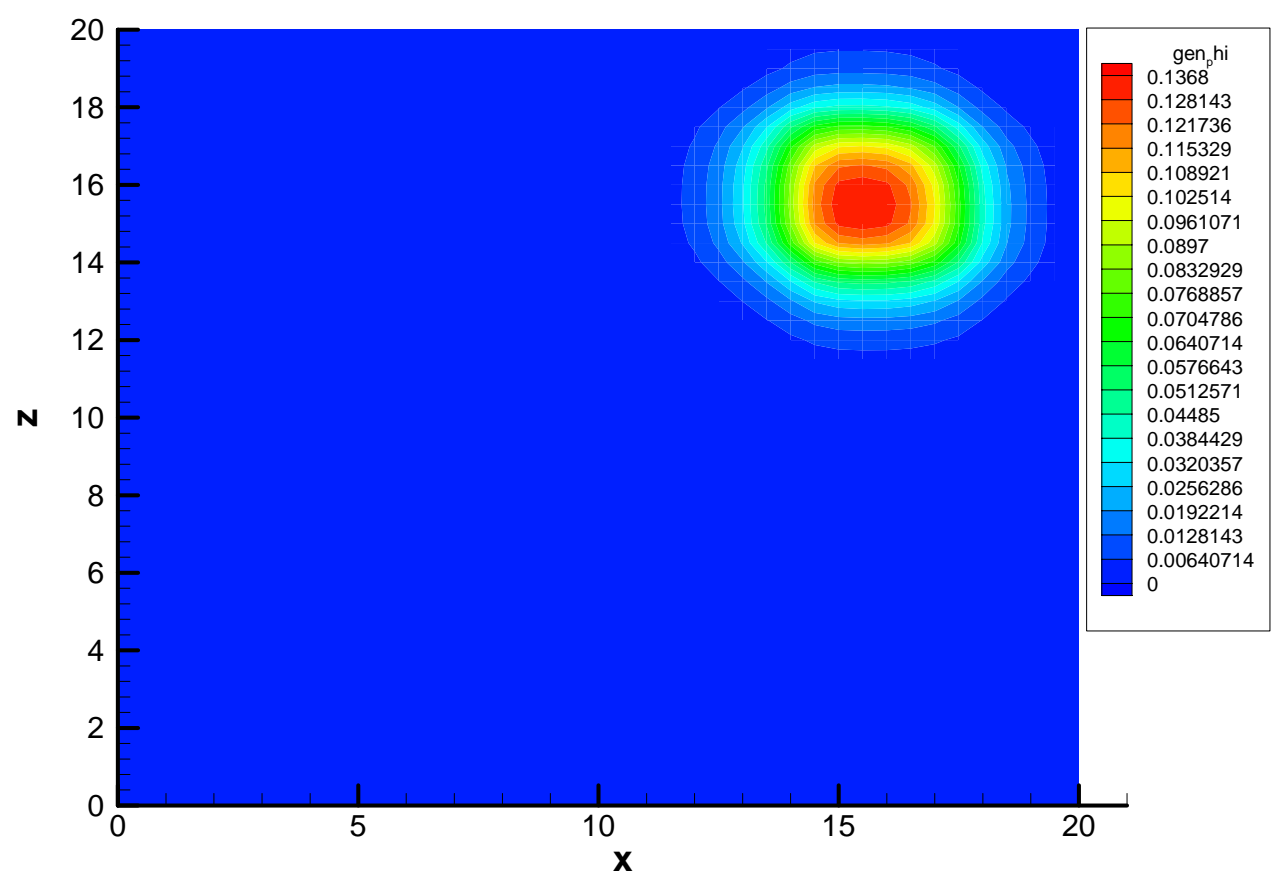

Figure A.3.6 - Results from transport of scalar quantity using QSOU3D in DREAM $^{\circledR}$

\section{Rotating Flow Field}

As the results shown thus far in comparison of the DREAM ${ }^{\circledR}$ simulation of a scalar transport quantity in an arbitrary flow field to those of $\mathrm{Hu}(2002)$, the DREAM ${ }^{\circledR}$ simulations compare very favorably to those of $\mathrm{Hu}$ (2002). Thus, the focus shifts to an interesting transport phenomena: Transient scalar transport in an imposed rotational (or vortex) flow field: In the specified square computation domain, each point moves around the center point $(0,0)$ with a constant angular velocity. The tangential velocity at each point can be simply calculated from $\omega r$, where $r$ is the distance from that point to the center (radius). As a result, every point provides a different $u$ velocity component and $v$ component, which in a Cartesian coordinate system should be a function of $x$ and $y$ position. Overall, the scalar signal inside the domain will be carried by the convective flow field and experiences a rotational motion around the center point. While the signal is rotating, it is also subject to a purely numerical diffusion process, which weakens the strength of the signal and makes it finally flat. Figures (A.3.7) and (A.3.8) show the results for this type of flow field, and once again, the simulation compare very favorably. 


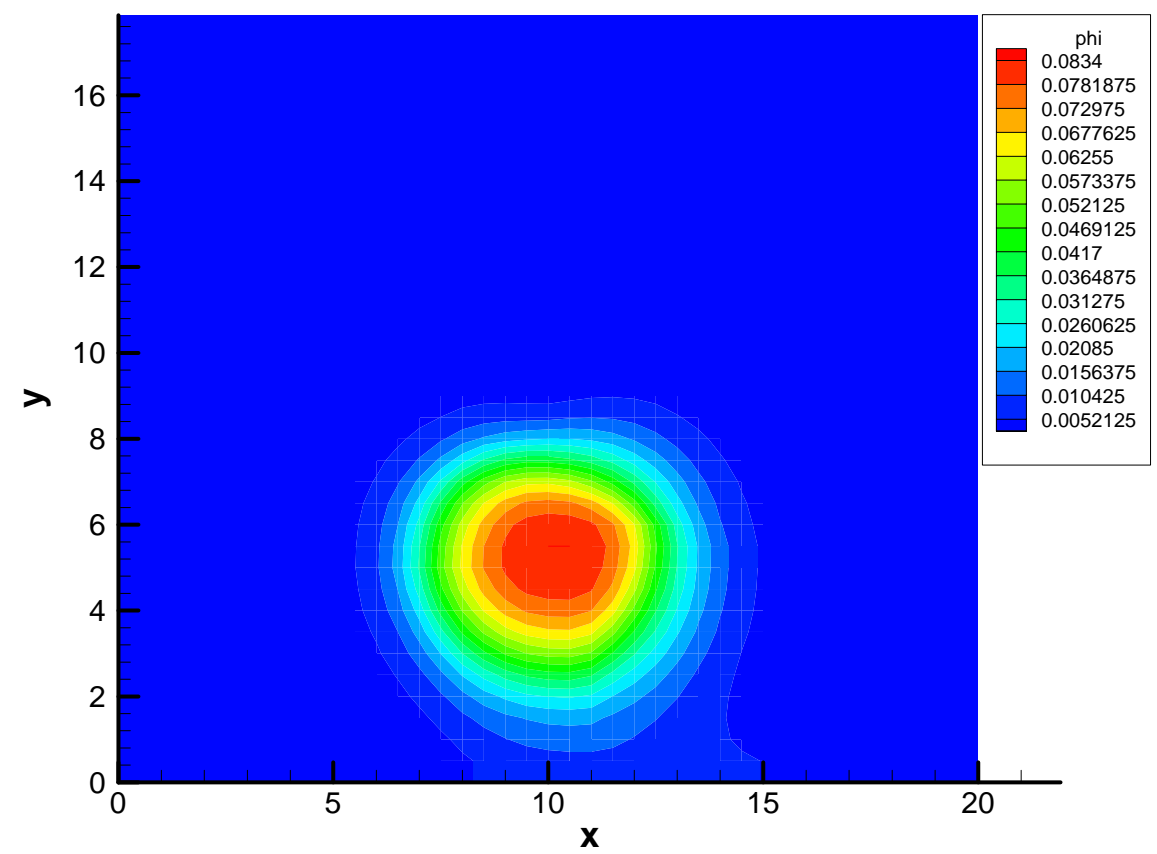

Figure A.3.7 - Results from transport of scalar quantity from Hu (2002) after 15 sec

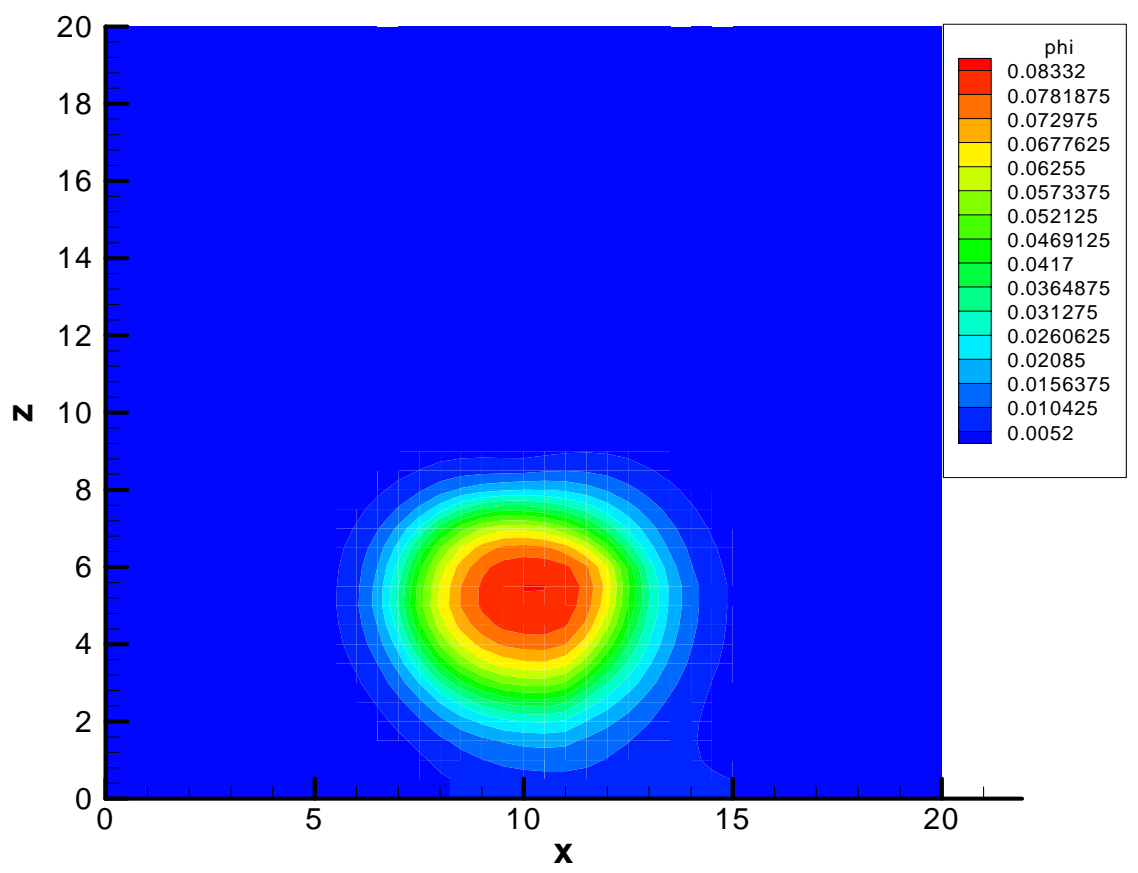

Figure A.3.8 - Results from transport of scalar quantity from DREAM ${ }^{\circledR}$ after 15 sec

Therefore, in conclusion, results from Figures (A.3.1) - (A.3.8) in a comparison with the simulations from $\mathrm{Hu}$ (2002) show that the results from implementation of QSOU into 
DREAM $^{\circledR}$ allow for accurate simulations of the transport of a generic scalar quantity into a known velocity field. The results of all of the simulations from DREAM ${ }^{\circledR}$ produce very similar results in magnitudes and pattern of the scalar quantity and the flow field. The magnitudes differ in the $10^{\wedge}-4$ digit generally in all of the flows, and it may therefore be concluded that the quasi-second order method has been properly implemented into $\operatorname{DREAM}^{\circledR}$. 


\section{A.4 Verification of Variable Density Scheme (Conservation of Mass)}

\section{Introduction}

In order to ensure the accuracy of the computer simulations performed using the variable density equations, conservation of mass is the crucial to the success of the study. At times, some CFD codes have difficulties in ensuring this, as they may not preserved monotonicity or positivity. As described in Section 3.0, DREAM ${ }^{\circledR}$ is formulated to ensure this.

For multiphase flows, the density is modeled using a mixture equation accompanied with an appropriate volume fraction equation. The QSOU scheme is used to determine an appropriate volume fraction of the mixture, and these values are then used to determine the appropriate density field.

\section{Verification}

In order to ensure that the numerical scheme conserves mass, the mass flow rate is used in the forcing of the conservation throughout the simulations. The geometry used is a standard vertical square pipe, being $0.55 \mathrm{~m} \times 2.0 \mathrm{~m} \times 0.55 \mathrm{~m}$ in size. An inlet velocity at the top is applied, with an outflow condition being applied at the bottom of the pipe. As conservation of mass is the only quantity being investigated in this study, only a laminar flow situation is being used. The downward inlet velocity is set to be -0.0025 $\mathrm{m} / \mathrm{s}$, which give a Reynolds number of approximately 200. No-slip velocities are specified at the walls. It is important to note that the initial velocity field is set equal to $0.0 \mathrm{~m} / \mathrm{s}$, meaning that as the fuel enters the pipe through the inlet, an initial shock of very small magnitude. The two fluids used in the verification are water, $\rho_{\mathrm{w}}=1000 \mathrm{~kg} / \mathrm{m}^{3}$, and fuel, $\rho_{\mathrm{f}}=850 \mathrm{~kg} / \mathrm{m}^{3}$. The density field is initialized as shown in Figure A.4.1. Using the specified velocity and density parameters, the theoretical tank filling time is 800 seconds. Simulations from DREAM ${ }^{\circledR}$ have a filling time of 803 seconds, so initially, the results compare qualitatively very well to theory. The grid used in the simulations was $40 \mathrm{x} 80 \mathrm{x}$ 40 grid cells, with a time step being 0.01 seconds. 
Since the initial velocity field is set equal to zero, a shock is caused throughout the domain as the simulations begin. These shocks are caused from the strict conservative scheme that is implemented into DREAM ${ }^{\circledR}$, as well as most control-volume, monotonic preserving schemes. It is important to note that the interface does not remain completely level in the axial or transverse direction. There are actually small fluctuations near the walls as lighter fluid pushes the heavier fluid downward causing a "rocking" motion between walls. However, these fluctuations are indeed small in magnitude. This phenomena may be clearly seen in Figure A.4.2.

The most important validation of any solver with variable density capabilities is in the ensuring of the conservation of mass. To check that mass is indeed conserved, the flow rates at the inlet and outlets are tracked. Results up to ten seconds are shown in Figure A.4.3, which allows for the initial oscillations to be shown. Clearly, because of the nature of the geometry and flow field conditions, the outlet mass flow rate is not equal to the inlet mass flow rate for the first 18 time steps. The propagation of the inlet information is not instantaneous, which causes the shocks to propagate downstream, thus the oscillatory convergence of the outlet flow rate. As the shocks diminish in magnitude, the velocity field stabilizes and conservation of mass is in fact achieved within 1.2 seconds after the simulations began. After this time, the error in the mass flow rate in and out of the system is $0.012 \%$.

Thus, in conclusion, it is clearly evident that the density formulation, in conjunction with the volume fraction equation, is conservative. Therefore, it may be concluded that provided with proper boundary conditions for each of the respective geometries, the overall mass within the system will be conservative. 


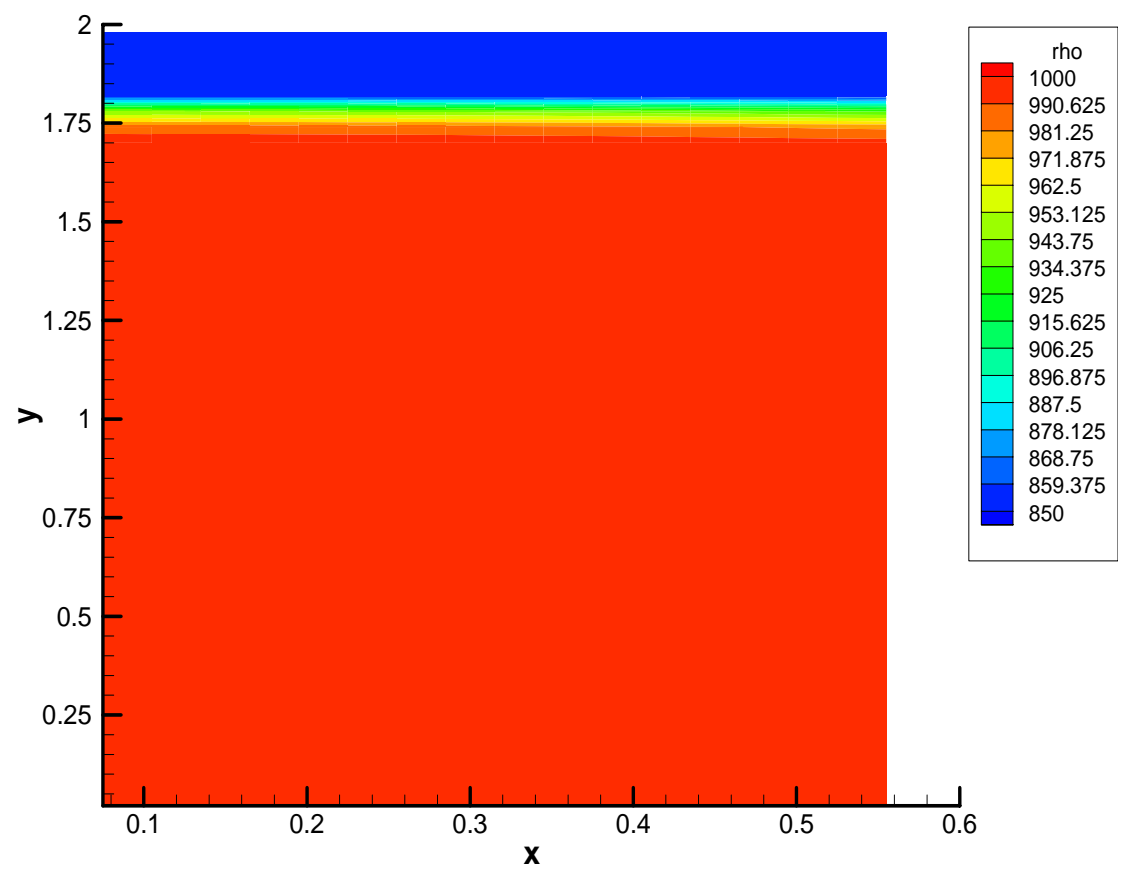

Figure A.4.1 - Initialization of density field for vertical tank filling, $t=0.0$ seconds

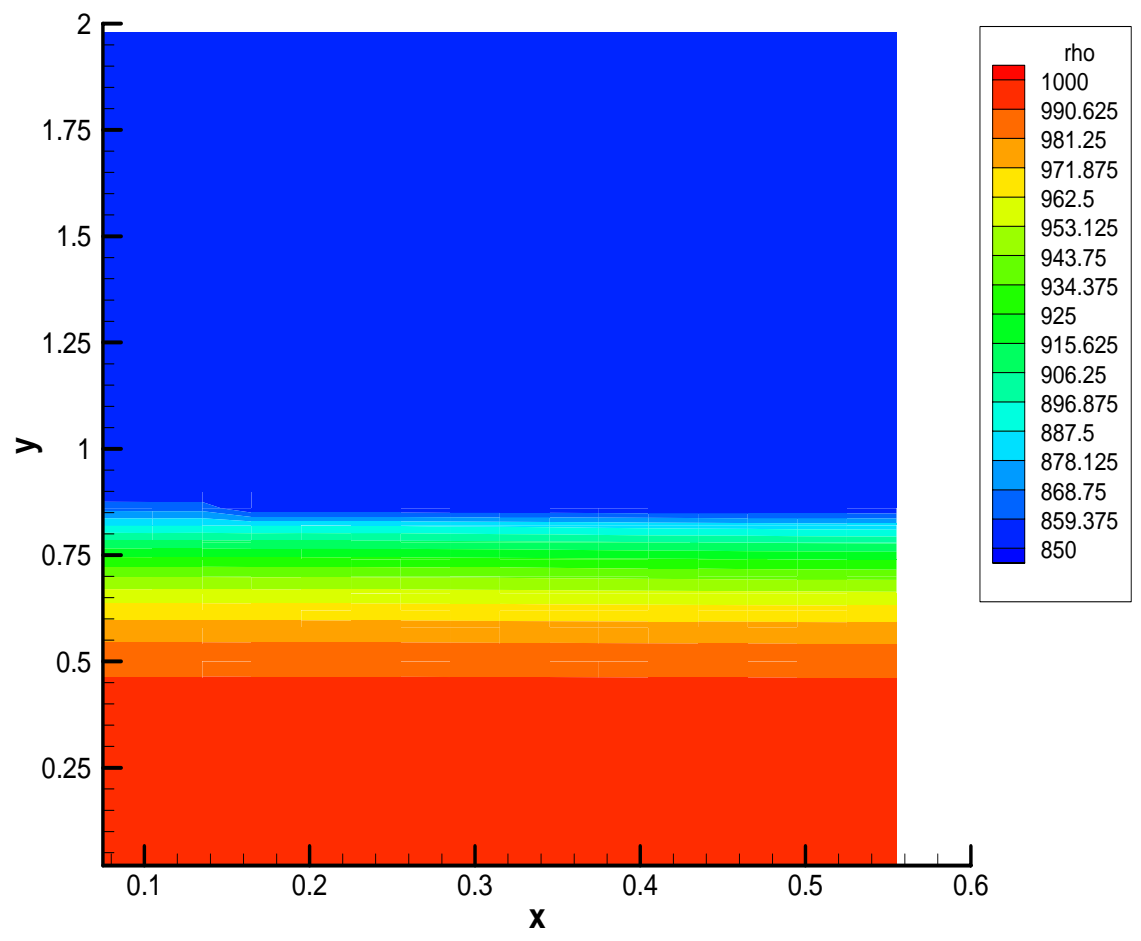

Figure A.4.2 - Density perturbations during flow for vertical tank filling with constant inlet velocity conditions, $t=\mathbf{5 7 5 . 0}$ seconds 


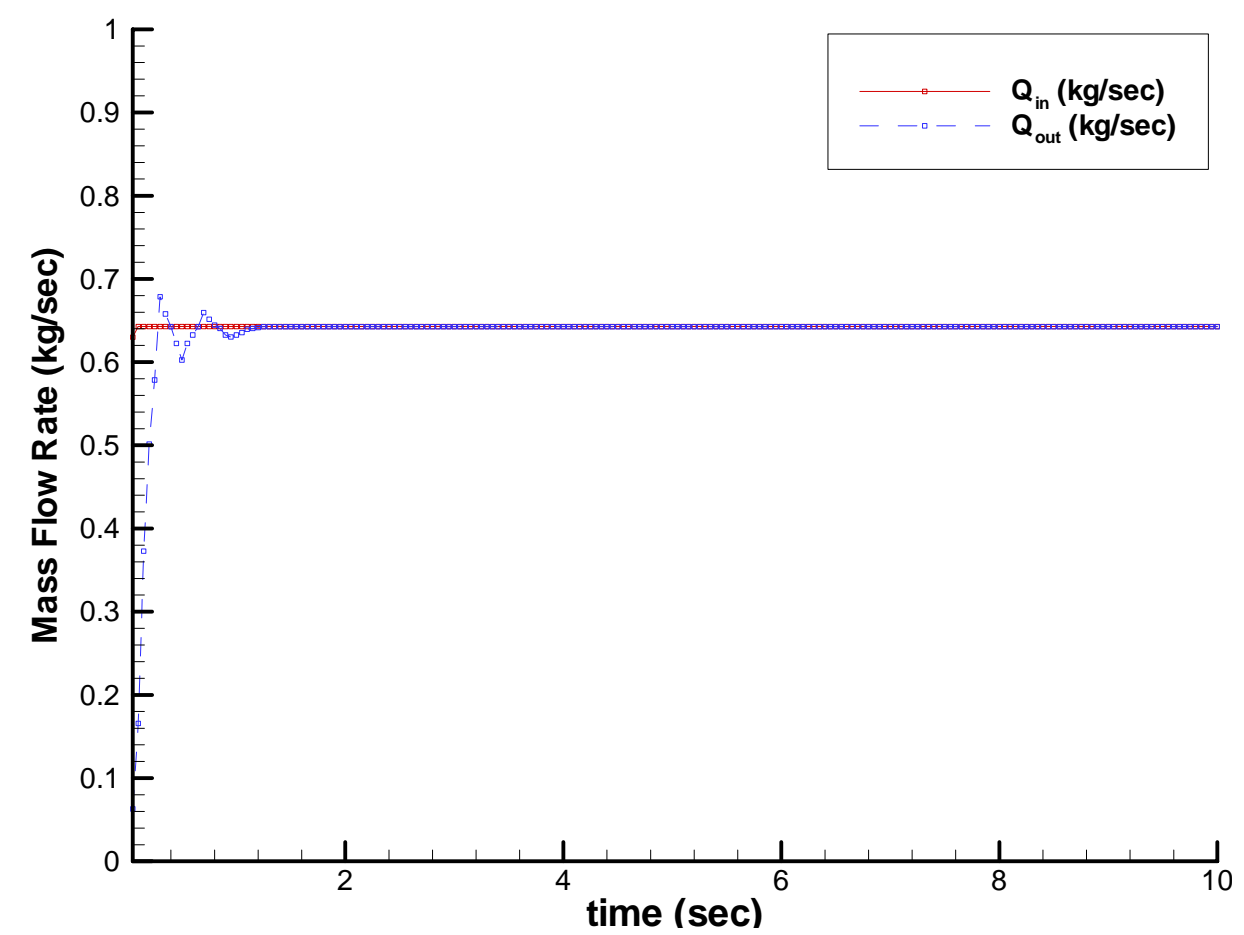

Figure A.4.3 - Mass flow rates at the inlet and outlet for first 10 seconds showing conservation of mass throughout the system 


\section{Appendix A.5 - Turbulence Analysis}

In order to determine the amount of turbulence present in all of the simulations, MATLAB was utilized to time average the calculated data to separate the mean and the fluctuating components of the instantaneous velocity data predicted from DREAM. The mean velocity is defined as

$$
\bar{u}(t)=\frac{1}{\Delta t_{\text {int }}} \int_{t-0.5 \Delta t_{\mathrm{int}}}^{t+0.5 \Delta t_{\mathrm{int}}} u\left(t^{\prime}\right) d t^{\prime}
$$

where $\mathrm{u}^{\prime}(\mathrm{t})$ is the fluctuating component of the velocity and $\Delta \mathrm{t}_{\mathrm{int}}$ is the averaging window of the data. A variety of window sizes were tested; however, for the data presented in this study, the most appropriate window size was found to be $1.5 \mathrm{msec}$. The fluctuating component of the velocity may be defined as

$$
u^{\prime}(t)=u(t)-\bar{u}(t)
$$

where $\mathrm{u}(\mathrm{t})$ is the instantaneous velocity data. The rms of the velocity is also solved, as follows

$$
u_{r m s}^{2}(t)=\frac{1}{\Delta t_{\text {int }}} \int_{t-0.5 \Delta t_{\text {int }}}^{t+0.5 \Delta t_{\text {int }}} u\left(t^{\prime}\right) u\left(t^{\prime}\right) d t^{\prime}
$$

To determine the relative turbulence intensities for the axial, vertical, and spanwise velocity components, the quantities are normalized relative to the axial mean flow velocity, $\mathrm{U}_{\mathrm{e}}$, giving

$$
\hat{u} \equiv \frac{\sqrt{u^{\prime 2}}}{U_{e}} ; \hat{v} \equiv \frac{\sqrt{v^{\prime 2}}}{U_{e}} ; \hat{w} \equiv \frac{\sqrt{w^{\prime 2}}}{U_{e}}
$$

The square of the relative turbulence intensities is also used in this study.

The allocation of the turbulent kinetic energy to motions of different length scales is called the turbulent energy spectrum, which indicates details about the turbulent motion within flows. This distribution of turbulent kinetic energy among motions of different length scales is defined as a function of the wave number, $\mathrm{K}$, which is inversely 
proportional to the eddy size. It is important to note that is some of the results presented, a frequency analysis is used as well. As mentioned earlier, MATLAB is used to solve for the energy spectrum, and the process is briefly summarized. The spectrum of a random process is mathematically related to the correlation sequence by the discrete-time Fourier transform given as

$$
S_{x x}(f)=\sum_{m=-\infty}^{\infty} R_{x x}(m) e^{-2 \pi j f m / f_{s}}
$$

where $f_{s}$ is the sampling frequency and $f$ is the physical frequency, with both being in units of hertz. $R_{x x}$ is the correlation sequence of the spectrum, which equals

$$
R_{x x}(f)=\int_{-f_{s} / 2}^{f_{s} / 2} \frac{S_{x x}(f) e^{-2 \pi j f m / f_{s}}}{f_{s}} d f
$$

Equation A.6.5 is used in the analysis of the velocity and density spectra of the data from the DREAM simulations. 


\section{APPENDIX B - INTRODUCTION TO DREAM ${ }^{\circledR}$ CODE}

The DREAM ${ }^{\circledR}$ code, as verified in this study, has been shown to be a robust and accurate LES code that requires no explicit SGS model. The Implicit Turbulence Model method has allowed for DREAM ${ }^{\circledR}$ to perform very well for the flows presented in this study. Written in Fortran, with instructions and comments within each subroutine, it is still worthwhile to summarize the code. As previously noted, DREAM utilizes a predictor-corrector, flux limiting scheme.

\section{B.1 Flow Chart}

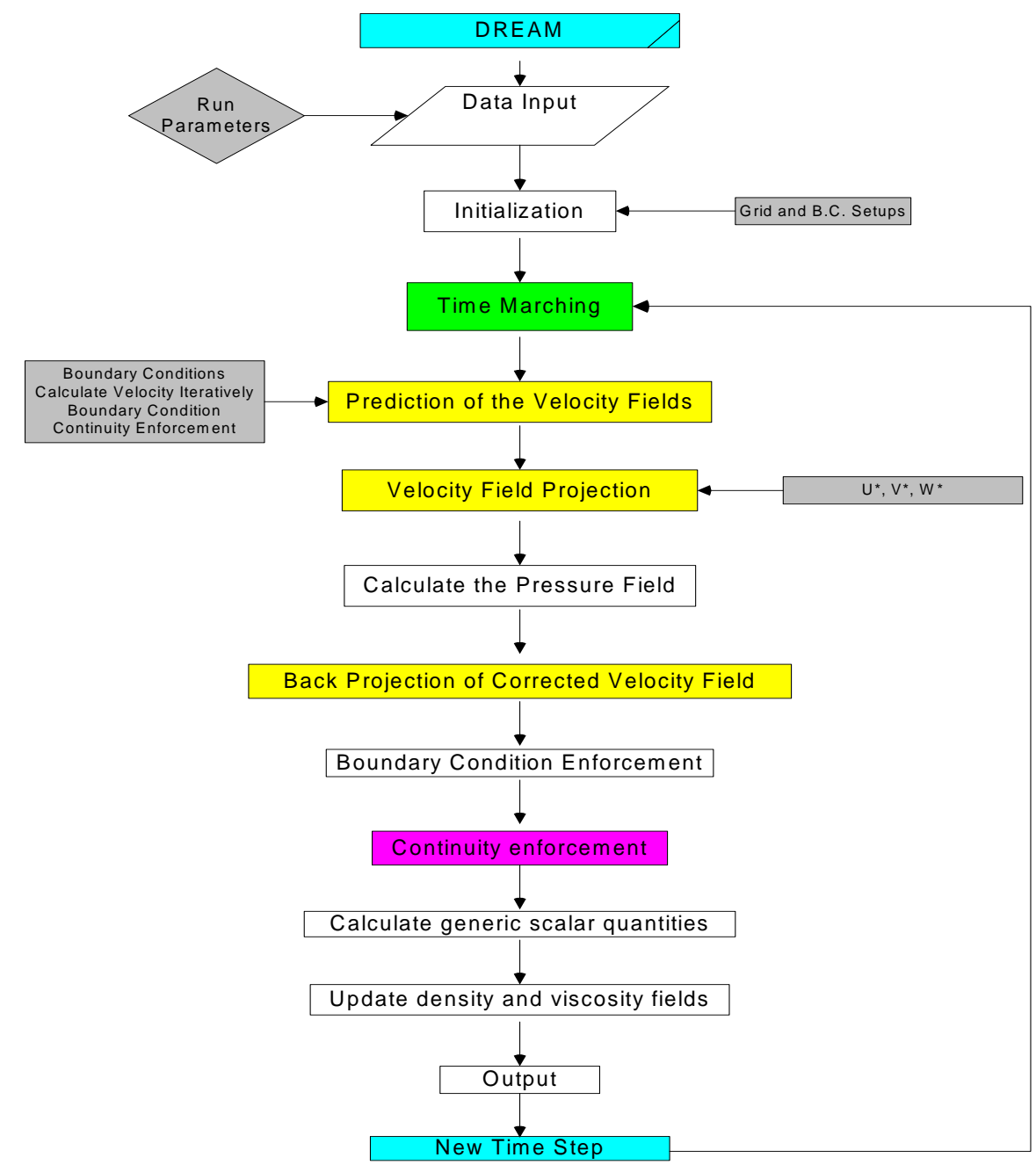

Figure B.1 - The flow chart of DREAM ${ }^{\circledR}$ for ITM LES 


\section{B.2 Inclusion of wall and baffles within domain}

In Chapter 3, the discretization scheme utilized in DREAM ${ }^{\circledR}$ enabling the solving of the Navier-Stokes equations was introduced. Equation 3.2.14 introduced the concept of linearization of the source term. This is done allowing for the incorporation of a linear dependence of the source, rather than treating it as a constant. Equation 3.2.14 was given by

$$
\bar{s}_{p}=\phi_{p} S_{p}+S_{c}
$$

It is important to note that the source term is usually the cause of a diverged solution, and that proper linearization is the key in obtaining a converged solution.

As mentioned previously, DREAM ${ }^{\circledR}$ is written to use a Cartesian grid, and the difficulty in allowing for internal walls and baffles must be handled properly. This is done through blocking off the cells within the regular grid so that the remaining grid forms the desired shape. Blocking off a cell is not as easy as setting the velocities in those locations equal to zero because this would improperly treat the matrix that needs to be solved, and doesn't handle the boundaries appropriately.

This operation consists of establishing known values of the relevant scalar variables in the inactive control volumes. This is accomplished by using very larger or small source terms, or the order of $10^{-30}$ or $10^{30}$, which in tern will dominate the discretization equation yielding

$$
\begin{aligned}
& s_{c}=10^{30} \phi_{p, \text { baffle }} \\
& s_{p}=10^{-30}
\end{aligned}
$$

where $10^{30}$ denotes a number large enough to make the other terms in the discretized equations negligible at that grid location. Thus, Eq. (3.2.14) would then become

$$
\begin{gathered}
\phi_{p, \text { baffle }} S_{p}+S_{c} \approx 0 \\
\therefore \phi_{p, b a f f l e}=-\frac{S_{c}}{S_{p}}
\end{gathered}
$$

Therefore, using this type of treatment of the source term, internal boundaries may be specified throughout the domain in Cartesian coordinates for DREAM ${ }^{\circledR}$ simulations. 


\title{
B.3 Description of the Subroutines
}

\author{
********************* Initialization and Setup $* * * * * * * * * * * * * * * * * * * * * * *$ \\ grid.for: $\quad$ staggard grid generation in Cartesian coordinate system. Domain \\ size is implemented in this location with units in meters
}

initial.for:

Sets up all initialization of velocity and scalar fields. Also, initial boundary conditions are included within this location

constants.for: All constants are defined in this location, including gravitational and implicitness factors for the solvers.

fc_cblock.for Defines all variables and allows for variables to be passes by defining only this common block, instead of individual variables.

fc_cparam.for Sets the maximum grid that may be run on the computer.

For $512 \mathrm{MB}$ machine, maximum grid size is approximately 1 million grid nodes

fc_cpsolve.for Sets up the common block parameters for the SIP3D, ICCG, and CGSTAB solvers

******************** DREAM ${ }^{\circledR}$ Navier-Stokes Solver *******************

Dream.for: $\quad$ Main program; opens and closes most files; all subroutine calls are made from this program; correction of the velocities and application of boundary conditions is within this subroutine

Calc_uvel_impl.for: Calculates the u velocity component; boundary conditions are applied within the code 
Calc_vvel_impl.for: Calculates the v velocity component; boundary conditions are applied within subroutine

Calc_wvel_impl.for: Calculates the w velocity component; boundary conditions are applied within subroutine

Interpolate.for: $\quad$ Interpolation scheme used for fully explicit schemes; User has option for central differencing, hybrid, upwinding first, third, and fifth order, central differencing scheme. Only written for uniform grids and must be modified for non-uniform grids

P_field.for: Calculates the pressure field through Poisson equation

Scalar_implicit.for: Contains the QSOU3D scheme and calculates the scalar transport variable (i.e. vof, temperature, etc.)

Properties.for: Calculates the densities and the viscosities based on the volume fractions

Slip_vel.for: $\quad$ Calculates a slip-velocity using a constant droplet diameter based on the calculated pressure gradients

Wall_functions.for: Calculates the $\mathrm{x}+, \mathrm{y}+, \mathrm{z}+$ wall units based on the law of the wall functions to determine $\mathrm{u}^{*}, \mathrm{v}^{*}, \mathrm{w}^{*}$

Bcond_vof.for: $\quad$ Sets the boundary conditions for the volume fraction calculations

Rhs_phi.for: The right hand side of each variable is computed using a general balance over a control volume and all field components may use this routine to calculate the r.h.s. 


\section{INDEX}

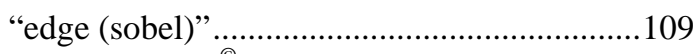

Adobe Premier ${ }^{\odot}$...............................................109

aliasing .................................................... 12

backscattering ............................................ 2

Bi-Conjugate Gradient Stabilized method.........35

buoyant jet... vi, 5, 16, 19, 20, 22, 23, 24, 61, 64, $107,110,111$

cascade .................................................... 8,62

Cole-Parmer.................................................... 108

compressional modes..............................13, 14

Computational fluid dynamics........................1

continuity.................................28, 32, 33, 34

control volume.. 2, 6, 8, 9, 12, 15, 26, 29, 39, 180

Crank-Nicholson scheme.................................12

densimetric Froude number.............................22

densimetric Froude number..20, 61, 62, 107, 108

dimensionless time ........................................ 111

dirichlet condition.............................................36

dissipation of turbulent kinetic energy ............xix

DNS ....................................................10, 13

DREAM ... 3, 4, 5, 6, 9, 15, 24, 26, 27, 32, 33, 34, $35,37,38,39,41,42,43,44,45,46,47,48$, $49,50,51,52,53,54,55,56,57,58,60,62$, $107,158,159,161,162,163,164,165,167$, $168,169,170,171,176,178$

energy spectrum ......................... $8,13,14,48$ enstrophy ........................................... 10, 14 entrainment............................16, 17, 21, 25, 61

filter ............................................................ 1,9

flux ..... 2, 8, 9, 11, 15, 17, 21, 23, 26, 28, 29, 30, $159,161,176$

conservation schemes .....................................

flux-limiting schemes ...................................... 9

Fourier spectrum............................................. 13

fractional step .................. See projection method

Grashof number ..............................................24

grid independence.......................................110

grid resolution..................................10, 12, 14

impingement.. 3, 5, 21, 23, 36, 61, 62, 65, 68, 69, 118

impinging jet.

$23,36,61$

Incomplete Cholesky Conjugate Gradient

Method .........................................................35

inertial subrange ..........................2, 12, 14, 48

irrotational.

ITM. xxi, 2, 3, 4, 5, 6, 8, 9, 10, 11, 12, 13, 14, 15, $24,26,27,30,31,32,36,37,39,45,46,48$, 62,176

jet penetration depth .65

Kelvin-Helmholtz ............................................48

Kolmogorov length scale. laminarization ................................................ 65

Large Eddy Simulation.......................... xxi, 1, 5

law of the wall .............................................. 12

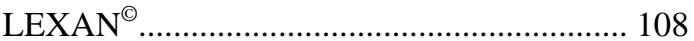

Mach number................................................ 13

MATLAB ................................................... 109

mean streamwise velocity distribution ............ 39

mixing layer.......................................45, 46

molecular viscosity ...................................... 13

momentum..... 1, 5, 15, 23, 24, 32, 33, 34, 61, 62

monotonicity ......................................2, 9, 170

Navier-Stokes equations ............ 1, 2, 5, 6, 10, 32

nonlinear monotone algorithms ..................... 10

no-slip boundary ....................................38, 46

Nusselt number .............................................. 24

positivity .................................2, 9, 11, 27, 170

QUICK scheme ..............................41, 42, 43

resolution $1,2,8,9,11,12,14,15,32,36,37,38$, 39,49

resolvable length scale. 48

Reynolds numberxvii, 11, 16, 18, 24, 25, 37, 170

Richardson extrapolation .......................110, 139

Richardson number........................ xvii, 24, 25

Runge-Kutta scheme.................................... 11

SGS...xxi, 2, 6, 10, 11, 12, 13, 14, 15, 32, 41, 42, 43, 176

shear layer..........................................10, 16, 47

similarity......... 108, 110, 111, 113, 115, 117, 124

Slip-wall boundary .................................... 46

Smagorinsky ...............10, 12, 13, 41, 42, 43, 46

solenoidal modes .......................................... 14

Square Cylinder .............................................. 37

Staggered grid................................................ 33

stagnant................................................ 108, 110

stratified .........................................5, 15, 20, 25

Strongly Implicit Procedure........................... 35

supersonic .......................................12, 13, 14

Taylor wave number................................... 14

temporal discretization ................................. 32

turbulence intensity.............................39, 42, 43

turbulence modeling ....................................5, 12

turbulent..xvii, xix, 1, 2, 4, 5, 6, 8, 10, 13, 14, 15, $17,19,21,22,23,24,26,37,39,45,46,47$,

48, 62, 107

turbulent kinetic energy ......................... 3, 8, 48 upwinding .......2, 3, 9, 11, 26, 158, 161, 163, 179 viscous diffusion........................................... 17 viscous length scaleSee Kolmogorov length scale vorticity ..............................12, 16, 17, 39, 44 wavelength .. 8 